CERN-TH.5540/89

\title{
TOPICS IN CONFORMAL FIELD THEORY
}

\author{
L. Alvarez-Gaumé and G. Sierra * \\ Theory Division, CERN \\ CH-1211 Geneva 23, Switzerland \\ C. Gomez ${ }^{\dagger}$ \\ Département de Physique Théorique, Université de Genève, Suisse
}

Contribution to the Knizhnik Memorial Volume to appear in World Scientific

Editors L. Brink, D. Friedan and A.M.Polyakov

* Address after October 1st 1989: Instituto de Estructura de la Materia, CSIC, Serrano 119, Madrid Spain

† Permanent, address: Departamento de Física, Universidad de Salamanca, Salamanca, Spain

CERN-TH.5540/89

September 1989 
TABLE OF CONTENTS

1. Introduction

2. General Properties of a Conformal Field Theory

2.1 Conformal Invariance and Conformal Ward Identities.

2.2 Conformal Blocks and Duality.

3. Degenerate Conformal Families. Coulomb Gas Representation

3.1 Generalities.

3.2 Coulomb Gas Representation Fusion Rules.

3.3 Virasoro Characters and Null Vectors.

3.4 Sample Computations Conformal Blocks and Braiding Matrices.

4. Current Algebras and Wess-Zumino-Written Models

4.1 Generalities.

4.2 Representations and Fusion Rules.

4.3 Partition Functions. Modular Invariants.

5. Fusion Algebras. The Verlinde Conjecture

5.1 The Fusion algebra and Its Properties.

5.2 Proof of The Verlinde Conjecture.

5.3 Constraints from Rationality.

6. Duality and Quantum Groups.
6.1 Motivation.
6.2 Quantum Groups and Their Representations, $q$ a Root of Unity.

7. Explicit Examples and Modular Properties

7.1 WZW Models.

7.2 Rational Gaussian Models.

7.3 Modular Properties.

8. Solvable Lattice Models and Conformal Field Theories 8.1 Vertex Models.

\footnotetext{
a.1. Regular Lattices.
a.2. Non-Regular Lattices. Z-Invariance.

a.1. Regular Lattices.
a.2. Non-Regular Lattices. Z-Invariance.
}

8.2 RSOS Models.

8.3 Relation to Conformal Field Theory and Quantum Groups.

9. Knots, Conformal Theories and Statistical Mechamincs 9.1 Knot Theory.

9.2 State Models. Turaev and Kauffunan Invariants.

9.3 Rational Conformal Theories and Knot Invariants.

9.4 Knots on Arbitrary Three Manifolds. 
central term of the Virasoro algebra from the assumption that the number of primary fields in the theory is finite ${ }^{19,20}$. Theories whose Hilbort space consists only of a finite number of representations are known as Rational Conformal Field Theories (RCFT) and they were introduced in Ref. 21 in the "Universal Moduli Space" formulation of CFT.

In Sec. 6 we concentrate on the duality properties of RCFT and motivate the use of quantum groups as the natural structure to characterize and classify $\mathrm{RCFT}^{22,23,24,25}$. The second part of this section gives a quick overview of the theory of Quantum Groups ${ }^{26,27,28,29,30}$ and their representations specially when the deformation parameters are roots of unity $31,32,25$. In Sec. 7 we apply the techniques of quantum groups to derive the duality properties of WZW models, Rational Gaussian Theories and one of the more interesting results in the connection between RCFT and quantum groups which computes using only properties of the co-multiplication the modular transformation matrix $S_{i j}$ determining the behavior of the characters under the modular transformation on the torus $\tau \rightarrow-1 / \tau^{25}$. This also allows us to obtain a proof of the Verlinde conjecture in this context and to connect with Witten's formulation of CFT and Knot and Link invariants in terms of Topological Field Theories in three dimensions ${ }^{33}$. In Sec. 8 we quickly review those aspects of integrable lattice models $34,35,36$ related to Conformal Field Theories and knot invariants. This section is included for completeness and also in preparation for Sec. 9 where we detail the connection between knot invariants, Conformal Field Theory and 3D Chern Simons gauge theories. We first present some of the basic notions in Knot Theory ${ }^{37,38}$ and connect with integrable models and Quantum Groups through Turaev's ${ }^{39}$ construction of Knot invariants in terms of extended Yang-Baxter systems. Next following Jones original work ${ }^{40,41}$ we show now the fusion rules of RCFT and the associated matrix $S$ generates a collection of Knot invariants. This will clarify what are the few ingredients of a RCFT needed to construct Knot polynomials regardless of how one wishes to study these theories in detail. In the last subsection in Sec. 9 we present the rudiments of Witten's construction of Knot invariants in arbitrary three manifolds ${ }^{33}$.

2. General Properties of a Conformal Field Theory

2.1 Conformal Invariance and Conformal Ward Identities

In this section we briefly review the basic properties of CFT in two 
dimension ${ }^{6}$ (see also Ref. 1). For an early application of conformal invariance in field theory, see Ref. 42. To avoid infrared problems we consider field theories on a cylinder $S^{1} \times \mathbb{R}$, described by coordinates $(\sigma, \tau), 0 \leq \sigma \leq$ $2 \pi,-\infty<\tau<+\infty$. The light cone coordinates are defined as $x^{ \pm}=\tau \pm \sigma$. Since the line element in two-dimensional Minkowski space has the form $d s^{2}=d x^{+} d x^{-}$, the group of transformations leaving the light cone invariant is infinite dimensional, and it is given by arbitrary reparametrizations of $x^{+}$and $x^{-}: x^{+}=f\left(x^{+}\right), x^{-}=g\left(x^{-}\right)$. In the analysis of CFT it is convenient to rotate to Euclidean space and use radial quantization. Under Euclidean rotation:

$$
\begin{aligned}
& \tau+\sigma \rightarrow-i(\tau+i \sigma)=-i w \\
& \tau-\sigma \rightarrow-i(\tau-i \sigma)=-i \bar{w}
\end{aligned}
$$

mapping now the cylinder to the plane.

$$
z=e^{w}
$$

we find that the $\tau=0$ "surface" becomes the unit circle on the $z$-plane $\tau=-\infty$ is mapped to $z=0$, and $r=+\infty$ to $z=+\infty$. The infinitesimal conformal transformations are generated by the vectors

$$
l_{n}=z^{n+1} \frac{d}{d z} \quad \bar{l}_{n}=\bar{z}^{n+1} \frac{d}{d \bar{z}} .
$$

A CFT is characterized by its scale invariace. As in any local field theory, scale invariance implies full conformal invariance. Scale invariance is equivalent to the vanishing of the trace of the energy-momentum tensor. In complex coordinates this means that $T_{z \bar{z}}=0$. Hence there are only two independent components of the energy-momentum tensor $T_{z z}, T_{z \bar{z}}$ and their conservation law becomes:

$$
\begin{array}{ll}
\partial^{z} T_{z z}=0 & \partial_{\bar{z}} T_{z z}=0 \\
\partial^{\bar{z}} T_{z z z}=0 & \partial_{z} T_{z z}=0
\end{array}
$$

implying that $T_{z z} \equiv T(z) \quad\left(T_{\tilde{z} \tilde{z}} \equiv \bar{T}(\bar{z})\right)$ is a holomorphic (antiholomorphic) function of $z(\bar{z})$. The generators of infinitesirnal conformal transformations are:

$$
L_{n}=\oint_{0} \frac{d z}{2 \pi i} z^{n+1} T(z)
$$

and similarly for $L_{n}$. The contour circles the origin only once. Its shape is irrelevant as a consequence of (2.3) and Cauchy's theorem. Using (2.4) we can write:

$$
T(z)=\sum_{n \in \mathbb{Z}} L_{n} z^{-n-2} \bar{T}(\bar{z})=\sum_{\nu \in \mathbb{Z}} L_{n} \bar{z}^{-n-2}
$$

Out of all the fields in the CFT we can distinguish some behaving like tensors under conformal transformations. These are called primary fields and they behave as $(h, \breve{h})$ tensors, i.e.

$$
\phi_{h, h}(z, \bar{z}) d z^{h} d \bar{z}^{h}
$$

is invariant under conformal transformations. If we use two different coordinates systems $z, z^{\prime}$, then $\phi_{h, h}$ transforms according to:

$$
\phi_{h, \bar{h}}^{\prime}\left(z^{\prime}, \bar{z}^{\prime}\right)=\phi_{h, \bar{h}}(z, \bar{z})\left(\frac{d z^{\prime}}{d z}\right)^{-h}\left(\frac{d \ddot{z}^{\prime}}{d \ddot{z}}\right)^{-\bar{h}}
$$

locality implies as we will see that $h-\bar{h}$ be an integer. Using (2.7) we can easily relate the mode expansions on the cylinder and on the plane. If we take for example $\bar{h}=0$ and $\phi_{n}$ a holomorphic field, the standard Fourier expansion on the cylinder is:

$$
\phi_{h}(w)=\sum_{n \in \mathbb{Z}} \phi_{n} e^{-n w}
$$

Using (2.1) and (2.7) we obtain the expansion on the plane:

$$
\phi_{h}(z)=\sum_{n \in \mathbb{Z}} \phi_{n} z^{-n-h}
$$

since the energy-momentum tensor has dimension $2, h=2$, and this also explains the expansion (2.5). The infinitesimal form of $(2.7)$ is:

$$
\begin{aligned}
& \delta_{\varepsilon} \phi_{h, \tilde{h}},=\left(\varepsilon(z) \frac{d}{d z}+h \varepsilon^{\prime}(z)\right) \phi_{h, h}=\left[T(z), \phi_{h, h}(z)\right] \\
& T(\varepsilon) \quad \equiv \oint_{0} \varepsilon(z) T(z)
\end{aligned}
$$


- (from now on the contour integral symbol includes $d z / 2 \pi i$ ). Equation (2.10) can be expressed more conveniently in terms of the operator product expansion (OPE) between $T(z)$ and $\phi(z, \bar{z})$. Since $T(z)$ is analytic, the commutator in $(2.10)$ is equivalent to a contour integral of $\varepsilon(w) T(w)$ around $\phi(z):$

$$
\left[T(\varepsilon), \phi_{h, h}(z)\right]=\oint_{z} \varepsilon(w) T(w) \phi_{h, h}(z, \bar{z})
$$

since the contour around $z$ can be chosen as small as we wish, the only contributions to (2.11) come from the singularities in the OPE. From (2.10) and (2.11) we obtain:

$$
T(z) \phi(w)=\frac{h}{(z-w)^{2}} \phi(w)+\frac{1}{z-w} \partial_{w} \phi(w)+\text { regular } .
$$

This is the operator form of the conformal ward identities. By the analyticity properties of $T(z)$ the correlation function

$$
\left\langle T(z) \phi_{1}\left(z_{1}\right) \ldots \phi_{N}\left(z_{N}\right)\right\rangle
$$

can be evaluated using (2.12). As a function of $z, T(z)$ is a meromorphic quadratic differential $(h=2)$ on the sphere. The only singularities appear at the points $z_{1}, \ldots, z_{N}$ and they are determined by the OPE (2.12). Hence,

$$
\begin{aligned}
\left\langle T(z) \phi_{1}\left(z_{1}\right) \ldots \phi_{N}\left(z_{N}\right)\right\rangle= & \sum_{j=1}^{N}\left(\frac{h_{j}}{\left(z-z_{j}\right)^{2}}+\frac{1}{z-z_{j}} \frac{\partial}{\partial z_{j}}\right) \\
& \left\langle\phi_{1}\left(z_{1}\right) \ldots \phi_{N}\left(z_{N}\right)\right)
\end{aligned}
$$

The "in" vacuum $|0\rangle$ can be represented by the insertion of the unit operator at the origin of the $z$-plane. If we consider the holomorphic vectors without poles at $z=0, z^{n+1} d / d z, n \geq-1$, and since the unit operator is a scalar, we conclude that:

$$
L_{n}|0\rangle=0 \quad n \geq-1
$$

as we will see later this ground state is invariant under the action of the Möbius group $\mathrm{SL}_{2}(\mathbb{C})$ of conformal isometries of the sphere. Taking Hermitian conjugates, $|0\rangle^{+}=\langle 0|, L_{n}^{+}=L_{m n}$, therefore the out vacuum $\langle 0|$ is invariant under the infinitesimal conformal transformations regular at $z=\infty$ :

$$
\langle 0| L_{-n}=0 \quad n \geq-1
$$

The primary states are defined by:

$$
|h, \ddot{h}\rangle=\lim _{z, \bar{z} \rightarrow+0} \phi_{h, \bar{h}}(z, \bar{z})|0\rangle
$$

Writing $(2.10)$ in components:

$$
\left[L_{n}, \phi_{n}(z)\right]=\left(z^{n+1} \frac{d}{d z}+(n+1) z^{n} h\right) \phi_{h}(z)
$$

we find that:

$$
\begin{aligned}
& L_{n}|h, \bar{h}\rangle=0 \quad n>0 \\
& L_{0}|h, \bar{h}\rangle=h|h, \bar{h}\rangle
\end{aligned}
$$

hence every primary field generates a highest weight representation of the conformal algebra $\left\{L_{n}\right\}$. The states of the representation $V_{h, \bar{h}}$ are constructed by acting on the primary state $|h, \tilde{h}\rangle$ with arbitrary polynomials in $\left\{L_{-n}, \vec{L}_{-m}, n, m \geq 1\right\}$. Such representations are known as Verma modules. In the operator language the states $L_{m n_{1}} \ldots L_{-n_{N}} \mid h, \hbar h>$ are represented as:

$$
\oint_{C_{1}} z_{1}^{-n_{1}+1} T\left(z_{1}\right) \oint_{C_{2}} z_{2}^{-n_{3}+1} T\left(z_{2}\right) \ldots \oint_{C_{N}} z_{N}^{-n_{N}+1} T\left(z_{N}\right) \phi_{h, \breve{h}}(z)
$$

where $C_{1} \supset C_{2} \supset \ldots \supset C_{N} \supset\{z\}$. The three generators $L_{ \pm 1}, L_{0}$ are associated to infinitesimal Möbius transformations. They are obtained from the vector fields $1, z, z^{2}$. These are the conformal killing vectors on the sphere. The integrated form of the infinitesimal transformations is the group of fractional linear transformations $\mathrm{SL}_{2}(\mathbb{C})$ :

$$
z^{\prime}=\frac{a z+b}{c z+d}, a, b, c, d \in \mathbb{C}, a d-b c=1
$$

thus $L_{-1}$ generates translations, $L_{0}$ generates scalar transformation, and $L_{+1}$ generates special conformal transformations, and in particular $|0\rangle$ is $\mathrm{SL}_{2}$ invariant. The $\mathrm{OPE}$ of $T(z)$ with itself is more complicated. The algebra generated by the vector fields $(2.2)$ :

$$
\left[l_{n}, l_{m}\right]=(m-n) l_{n+m}
$$


is not realized faithfully but projectively. This should be expected because in quantum mechanics the physical states are described by rays on a Hilbert space.

The central extension of (2.21) in the projective representation generated by the energy-momentum tensor is determined by the two point function of $T(z)$. By dimensional analysis and $\mathrm{SL}_{2}$ invariance of $\mid 0>$ together with the requirement that the energy-momentum tensor should vanish as $z \rightarrow \infty$ (no sources at $\infty$ ) we obtain:

$$
\langle 0|T(z) T(w)| 0\rangle=\frac{c / 2}{(z-w)^{4}}
$$

the constant $c$ depends on the CFT. Hence the OPE between two T's is given by:

$$
T(z) T(w)=\frac{c / 2}{(z-w)^{4}}+\frac{2}{(z-w)^{2}} T(w)+\frac{1}{z-w} \partial_{w} T(w)+\text { regular }
$$

implying that under infunitesimal transformation:

$$
\begin{aligned}
\delta_{\varepsilon} T(z) & =[T(\varepsilon), T(z)]=\oint_{z} \varepsilon(w) T(w) T(z) \\
& =\left(\varepsilon(z) \frac{d}{d z}+2 \varepsilon^{\prime}(z)\right) T(z)+\frac{c}{12} \varepsilon^{\prime \prime \prime}(z)
\end{aligned}
$$

or in terms of $L_{n 2}$ commutators.

$$
\left[L_{n}, L_{m}\right]=(n-m) L_{n+M}+\frac{c}{12}\left(n^{3}-n\right) \delta_{n+m, o}
$$

and we obtain the Virasoro algebra. It is important to notice that the central terms in (2.25) vanishes for $n=0, \pm 1$. This is reflected in the $\mathrm{SL}_{2}$ invariance of the ground state. Equivalently, the inhomogeneous terms in (2.24) vanishes for $\varepsilon=1, z, z^{2}$. This is useful in determining the finite transformation rule of $T(z)$ under conformal maps. Using the properties of the fractional linear transformations, one can show that under a finite transfromation $z=f(w)$ :

$$
\begin{aligned}
& T^{\prime}(w)=\left(\frac{d z}{d w}\right)^{2} T(z)+\frac{c}{12} S(z, w) \\
& S(z, w)=\frac{\left(z^{\prime} z^{\prime \prime \prime}-\frac{3}{2}\left(z^{\prime \prime}\right)^{2}\right)}{z^{\prime 2}}
\end{aligned}
$$

$S$ is the schwarzian derivative and it vanishes when $z$ is a Mobius transformation of $w$. As an example, we can compute the relation between $T$ in the cylinder $T_{\text {cyl }}(w)$ and $T(z)$. Substituting $(2.1)$ in $(2.26)$ we obtain:

$$
\begin{aligned}
& T_{\text {cyl }}(w)=z^{2} T(z)-\frac{c}{24} \\
& T_{\text {cyl }}(w)=\sum_{n \in \mathbb{Z}} L_{n} z^{-n}-\frac{c}{24}=\sum_{n \in \mathbb{Z}}\left(L_{n}-\frac{c}{24} \delta_{n, 0}\right) e^{-n w} \\
& \left(L_{0}\right)_{\text {cyl }}=L_{o}-\frac{c}{24}
\end{aligned}
$$

and $c$ can be interpreted as a Casimir energy. Hence if we want to compute the physical partition function, we learn from (2.27) that:

$$
z=\operatorname{tr} e^{-\beta\left(L_{0}+\bar{L}_{0}\right) c y l}=\operatorname{tr} e^{-\beta\left(L_{0}+\bar{L}_{0}-c / 12\right)},
$$

The simplest examples of conformal theories are free scalars and free Weyl-Majorana fermions. The energy-momentum tensor of a free scalar is:

$$
T(z)=-\frac{1}{2}: \partial \phi(z) \partial \phi(z):, \partial \phi(z)=\sum_{n \in \mathbb{Z}} \alpha_{n} z^{-n-1}
$$

with the basic two-point function:

$$
\partial \phi(z) \partial \phi(w)=\frac{-1}{(z-w)^{2}}+: \partial \phi(z) \partial \phi(w):
$$

and one easily obtains $c=1$. For a Weyl-Majorana fermion there are two possibilities. We can have periodic or antiperiodic boundary conditions on the cylinder. Since in this case $h=1 / 2$, we learn from (2.7) that a periodic (antiperiodic) fermion on the cylinder becomes an antiperiodic (a periodic) fermion on the plane. These boundary conditions are known respectively as Ramond (R) and Neveu-Schwarz (NS) boundary conditions:

$$
\begin{aligned}
& \psi\left(e^{2 \pi i} z\right)=\psi(z) \\
& \psi\left(e^{2 \pi i} z\right)=-\psi(z)
\end{aligned}
$$

with associated mode expansions:

$$
\begin{aligned}
& \psi(z)=\sum_{n \in \mathrm{Z}+1 / 2} \psi_{n} z^{-n-1 / 2} \\
& \psi(z)=\sum_{n \in \mathbb{Z}} \psi_{n} z^{-n-1 / 1}
\end{aligned}
$$


a and we see that in the Ramond sector $\psi(z)$ has a square root cut from 0 to $\infty$. The canonical anticommulatation relations are:

$$
\left\{\psi_{n}, \psi_{m}\right\}=\delta_{n+m}
$$

and there is a zero mode in the Ramond sector. The singularly on the OPE is

$$
\psi(z) \psi(w)=\frac{1}{z-w}+\text { regular }
$$

in both cases,

$$
T(z)=\frac{1}{2}: \psi(z) \partial_{z} \psi(z):
$$

and $c=1 / 2$.

The symmetry of a CFT under the Virasoro algebra $V \otimes \bar{V}$ means that the operators (or states) of the theory fall into different conformal families, each generated by a primary field. The states generated by the action of $T(z)$ on the primary field (2.11), known as descendent fields; are determined by those of the primary fields through the use of the conformal Ward identity (2.14). As an example we compute:

$$
\begin{aligned}
& \left\langle\left(L_{-n} \phi\right)(z) \phi_{1}\left(z_{1}\right) \ldots \phi_{N}\left(z_{N}\right)\right\rangle \\
& =\left\langle\oint_{z}(w-z)^{-n+1} T(w) \phi(z) \phi_{1}\left(z_{1}\right) \ldots \phi_{N}\left(z_{N}\right)\right\rangle
\end{aligned}
$$

deforming the contour so that it circles $z_{1} \ldots z_{N}$ and using the OPE (2.12) (assuming all $\phi_{i}\left(z_{1}\right)$ are primaries) one obtains:

$$
(-1)^{n-1} \sum_{i=1}^{N}\left(\frac{(1-n) h_{i}}{\left(z-z_{i}\right)^{n}}+\frac{1}{\left(z-z_{i}\right)^{n-1}} \frac{\partial}{\partial z_{i}}\right)\left\langle\phi(z) \phi_{1} \ldots \phi_{N}\right\rangle .
$$

Notice also that

$$
\left(L_{-1} \phi\right)(z)=\frac{\partial \phi}{\partial z}
$$

Hence to proceed further we need to find the general properties of correlation functions of primary fields. In the next subsection we will use the associativity of the OPE to derive the duality properties of 4-point functions. We conclude this subsection by analyzing the general $\mathrm{SL}_{2}$ invariance properties of the correlation functions ${ }^{6}$. Since $L_{ \pm 1}|0\rangle=L_{0}|0\rangle=0$ we immediately obtain

$$
\begin{aligned}
\sum_{i=1}^{N} \quad \frac{\partial}{\partial z_{i}}\left\langle\phi_{1}\left(z_{1}\right) \ldots \phi_{N}\left(z_{N}\right)\right\rangle & =0 \\
\sum_{i=1}^{N}\left(z_{i} \frac{\partial}{\partial z_{i}}+h_{i}\right)\left\langle\phi_{1}\left(z_{1}\right) \ldots \phi_{N}\left(z_{N}\right)\right\rangle & =0 \\
\sum_{i=1}^{N}\left(z_{i}^{2} \frac{\partial}{\partial z_{i}}+2 h_{i} z_{i}\right)\left\langle\phi_{1}\left(z_{1}\right) \ldots \phi_{N}\left(z_{N}\right)\right\rangle & =0
\end{aligned}
$$

and similarly for $L_{ \pm 1}, L_{0}$ (for simplicity of notation we present most of the formulae only for the explicit dependence on $z_{1} \ldots z_{N}$ ). The behavior of the correlation functions under finite $\mathrm{SL}_{2}$ transformations follows from (2.7) and (2.38):

$$
\begin{gathered}
g(z)=\frac{a z+b}{c z+d} \quad \frac{d g}{d z}=\frac{2}{(c z+d)^{2}} \\
\left\langle\phi_{1}\left(g\left(z_{1}\right)\right) \ldots \phi_{N}\left(g\left(z_{N}\right)\right)\right\rangle=\left(\prod_{i=1}^{N}\left(c z_{i}+d\right)^{2 h_{i}}\right)\left\langle\phi_{1}\left(z_{1}\right) \ldots \phi_{N}\left(z_{N}\right)\right\rangle
\end{gathered}
$$

given any four points $w_{1}, w_{2}, w_{3}, w_{4}$ we can construct an $\mathrm{SL}_{2}$ invariant in terms of the harmonic ratio:

$$
\eta=\frac{w_{12} w_{34}}{w_{14} w_{32}}, w_{i j} \equiv w_{i}-w_{j}
$$

and we can write $\left\langle\phi_{1} \ldots \phi_{N}\right\rangle$ in terms of a function of $N-3$ harmonic ratios times a prefactor which takes care of the transformation property $(2.39)$. Using

$$
z_{i}-z_{j} \rightarrow g\left(z_{i}\right)-g\left(z_{j}\right)=\frac{z_{i j}}{\left(c z_{i}+d\right)\left(c z_{j}+d\right)}
$$

we write:

$$
\left\langle\phi_{1}\left(z_{1}\right) \ldots \phi_{N}\left(z_{N}\right)\right\rangle=\left(\prod_{i<j} z_{i j}^{-\gamma_{i j}}\right) f\left(\eta_{a}\right) \quad a=1, \ldots, N-3
$$

to determine the $\gamma_{i j}$ we impose (2.39) on the prefactor. Then:

$$
\begin{aligned}
\gamma_{i j} & =\gamma_{j i} \\
\sum_{j \neq i} \gamma_{i j} & =2 h_{i}
\end{aligned}
$$


In particular, the two- and three-point functions are completely determined:

$$
\begin{aligned}
& \left\langle\phi_{h, \bar{h}}(z, \bar{z}) \phi_{h^{\prime} h^{-1}}(z w, \bar{w})\right\rangle=a \delta_{h, h^{\prime}} \delta_{\bar{h} \bar{h}^{\prime}}(z-w)^{-2 h}(\bar{z}-\bar{w})^{-2 \tilde{h}} \\
& \left\langle\phi_{1}\left(z_{1}\right) \phi_{2}\left(z_{2}\right) \phi_{3}\left(z_{3}\right)\right\rangle=C_{123} z_{22}^{-\gamma_{12}} z_{13}^{-\gamma_{13}} z_{23}^{-\gamma 2 s} \cdot \text { (antihol. part) } \\
& \gamma_{i j}=h_{i}+h_{j}-h_{k}
\end{aligned}
$$

The only nontrivial dynamical information is the coefficient $C_{123}$ which determines the operator product expansion coefficients for the CFT. Know edge of these coefficients is equivalent to knowing the CFT. The equations satisfied by $C_{123}$ are presented next.

\subsection{Conformal blocks and duality}

To find the crossing or duality transformation satisfied by the constants $C_{i j k}$ we study the four-point function. We define the out state created by a primary field as:

$$
\langle h, \bar{h}|=\lim _{z, \bar{z} \rightarrow \infty}\langle 0| \phi_{h, \bar{h}}(z, \ddot{z}) z^{2 L_{0}} \bar{z}^{2 L_{0}}
$$

This is the definition induced by the scalar product defined by the two point function (2.43). With this definition we can simplify (2.43):

$$
\left\langle n\left|\phi_{m}(z, \bar{z})\right| l\right\rangle=C_{n m}^{l} z^{h_{n}-h_{m}-h_{l}} \bar{z}^{\bar{h}_{n}-\breve{h}_{m}-\bar{h}_{l}}
$$

and in the four-point function we can choose three points to be $0,1, \infty$. Hence we are interested in:

$$
\left\langle\left|l \phi_{l}(1,1) \phi_{n}(x, \ddot{x})\right| m\right\rangle
$$

If we consider the OPE of two primary fields:

$$
\begin{aligned}
& \phi_{n}(z, \bar{z}) \phi_{m}(0,0)=\sum_{p} \sum_{\{k, \bar{k}\}} C_{n m}^{p\{k, \bar{k}\}} . \\
& z^{h_{p}-h_{n}-h_{m}+\Sigma k} \bar{z}^{\bar{h}_{p}-\bar{h}_{n}-\vec{h}_{m}+\Sigma \bar{k}} \phi_{p}^{\{k, \bar{k}\}}(0,0)
\end{aligned}
$$

The index $p$ runs over the primary fields, and $k, \bar{k}$ label descendants, $k$ is a collection of integers $k_{1}, \ldots k_{N} \geq 0$ and

$$
\phi_{p}^{\{k, \bar{k}\}}(0)=L_{-k_{1}} \ldots L_{-k_{N}} \ldots L_{-\vec{k}_{M}} \phi_{p}(0,0)
$$

from conformal invariance,

$$
C_{n, m}^{p\{k, \bar{k}\}}=C_{n m}^{p} \beta_{n m}^{p\{k\}} \beta_{n m}^{-\tilde{p}\{\bar{k}\}}
$$

and the $\beta$-coefficients can be calculated using the conformal Ward identities in terms of the conformal dimensions and the central extension $c$ of the Virasoro algebra (see [BPZ] for details). Substituting (2.47) in (2.46):

$$
\begin{aligned}
& \left\langle k\left|\phi_{l}(1,1) \phi_{n}(x, \bar{x})\right| m\right\rangle \\
& =\langle k| \phi_{l}(1,1) \sum_{p} C_{n m}^{p} x^{h_{p}-h_{n}-h_{m}} \bar{x}^{h_{p}-h_{n}-h_{m}} \sum_{\{k, \bar{k}\}} \beta_{n m}^{p\{k\}} \beta_{n m}^{p\{k\}} \\
& \times x^{\Sigma k} \bar{x}^{\Sigma \bar{k}} \phi_{p}^{\{k, k\}}(0,0)|0\rangle
\end{aligned}
$$

defining,

$$
\xi_{n m}^{l k}(p \mid x)=x^{h_{p}-h_{n}-h_{m}} \sum_{\{k\}} \beta_{n m}^{p\{k\}} \frac{\left\langle k\left|\phi_{1}(1,1) L_{-k_{1}} \ldots L_{-k_{N}}\right| p\right\rangle}{\left\langle k\left|\phi_{l}(1,1)\right| p\right\rangle} \cdot x^{\Sigma k}
$$

The Green's function becomes:

$$
G_{n m}^{l k}(x, \bar{x})=\sum_{p} C_{n m}^{p} C_{k l p} \xi_{n m}^{l k}(p \mid x) \overline{F_{n m}^{l k}(p \mid x)}
$$

Graphically each function $\mathcal{F}$ is represented as a skeleton diagram

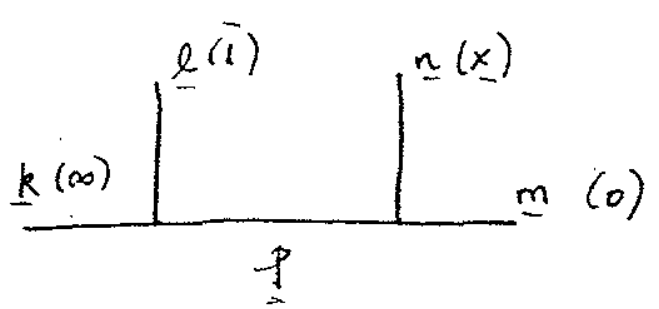

The generally multivalued functions $F(p \mid x)$ are known as the conformal blocks. The blocks are normalized so that when $x \rightarrow 0$ :

$$
F_{n m}^{l k}(p \mid x) \simeq x^{h_{p}-h_{n}-h_{m}}(1+\ldots)
$$

This is the convention chosen in Ref. 6. Requiring associativity of the OPE gives us the desired equations for $C$ : the bootstrap, duality or crossing 
relations. The skeleton in $(2.53)$ indicates the order in which we perform the operator product: we first do the OPE for $n$ with $m$ and then do the expansion with $l$. Associativity implies that the physical amplitude should not depend on whether we do first the OPE between $n, m$ or $l, n$. Use first the $\mathrm{SL}_{2}$ transformation $x \rightarrow 1-x$ to obtain $\left\langle k\left|\phi_{n}(1,1) \phi_{n}(1-x, 1-\bar{x})\right| l\right\rangle$ hence $G_{n m}^{l k}(x, \bar{x})=G_{n l}^{m k}(1-x, 1-\bar{x})$ similarly with $x \rightarrow 1 / x$. We thus obtain:

$$
G_{n m}^{l k}(x, x)=G_{n l}^{m k}(1-x, 1-\tilde{x})=x^{-2 h_{n}} \bar{x}^{-2 \bar{h}_{n}} G_{n k}^{l m}\left(\frac{1}{x}, \frac{1}{\bar{x}}\right)
$$

now do the OPE between $l, n$ first. Following steps similar to those leading to $(2.52)$ we arrive at:

$$
\begin{aligned}
& \sum_{p} C_{n m}^{p} C_{k l p} \mathcal{F}_{n m}^{l k}(p \mid x) \overline{F_{n m}^{l k}(p \mid x)} \\
& =\sum_{q} C_{n l}^{q} C_{k m q} \mathcal{F}_{n l}^{m k}(q \mid 1-x) \overline{F_{n l}^{m k}(q \mid 1-x)}
\end{aligned}
$$

since $\bar{F}, \bar{F}$ are determined by the conformal dimensions $h$ and the conformal Ward identities; equation (2.56) gives a rather nontrivial set of conditions characterizing the possible operator product coefficients which may appear in CFT. The problem of classifying all CFTs in two dimension is equivalent to solving (2.56) for all possible $c$ (with some extra constraints coming from modular invariance). We can represent (2.56) graphically as:

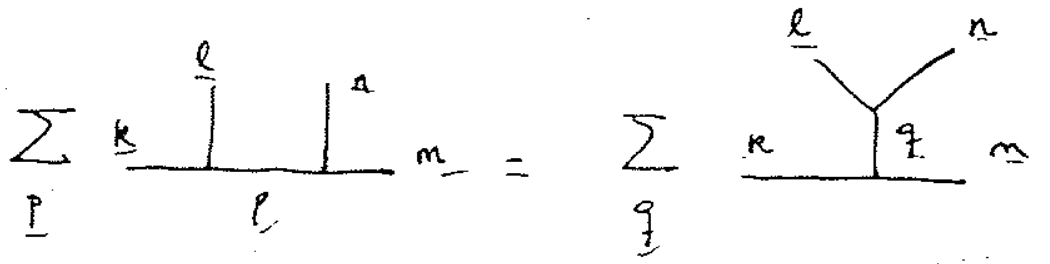

exhibiting the crossing symmetry implied by (2.56).

It is worthwhile stressing that the two basic properties we have used so far of CFT are: 1) Locality, 2) Associativity of the OPE. Locality implies that although the blocks are in general multivalued, the physical correlation functions have trivial monodromy. Thinking of the blocks as section of a vector bundle, the monodromy invariant metric is constructed in terms

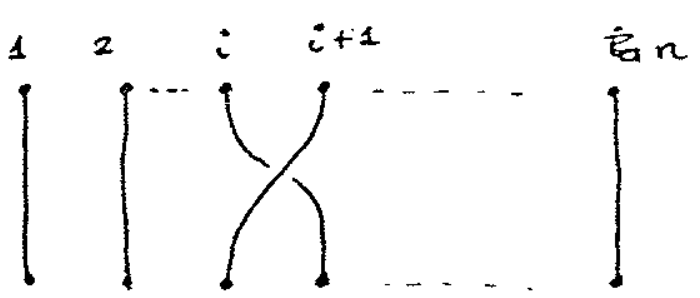

Fig. 1. Generator $\sigma_{i}$ of the braid group on n strands $\mathrm{B}_{n}$

of the structure constants of the operator algebra. The general physical correlation function $G_{i_{1} \ldots i_{N}}\left(z_{1} \ldots z_{N}\right)$ can also be expressed in terms of more general blocks,

$$
\frac{\left.\left.\left.\left.i_{1}\right|_{-p_{1}} ^{i_{2}}\right|^{i_{3}} \ldots\right|^{i_{N-2}}\right|^{i_{N-1}}}{-P_{N-3}}=F^{i_{1} \ldots i_{N}} P_{1} \ldots P_{N-3}(z)
$$

the physical correlator is the monodromy invariant combination of $\xi_{\mathrm{p}}$ and $\overline{\bar{F}}_{\mathbf{p}^{\prime}}$ :

$$
\begin{aligned}
& G^{i_{1} \ldots i_{N}}\left(z_{1}, \bar{z}_{1}, \ldots, z_{N}, \bar{z}_{N}\right) \\
& \quad=\sum_{\mathbf{p}, \mathbf{p}^{\prime}} h_{\mathrm{p}, \mathbf{p}^{\prime}}{\xi_{\mathrm{p}}}^{i_{1} \ldots i_{N}}\left(z_{1}, \ldots\right) \overline{\mathcal{F}_{\mathrm{p}}^{i_{1} \ldots i_{N}}\left(z_{1}, \ldots\right)}
\end{aligned}
$$

and the invariant metric $h_{\mathbf{p}, \mathbf{p}^{\prime}}$ is built out of the structure constants $C_{i j}^{k}$. Locality implies that $G$ is independent of the order of its arguments. They can be exchanged without changing $G$. For blocks, the same certainly does not apply. Since $\mathcal{F}$ is in general multivalved we can define the $\mathcal{F}$ 's for some ordering of its arguments (for example $\left|z_{1}\right|>\left|z_{2}\right|>\ldots$ ) and then analytic continuation relates blocks in different domains. This is the question of monodromy and half-monodromy (braids) transformations.

Since analytic continuation depends on the path chosen to perform it; we find that it is the braid group rather than the permutation group which acts on the blocks. The braid group on $n$ strands $B_{n}$ is defined by $n-1$ generators $\sigma_{i}, i=1, \ldots, n-1$ shown in Fig. 1 , and two relations ${ }^{7}$ :

$$
\begin{array}{ll}
\sigma_{i} \sigma_{j} & =\sigma_{j} \sigma_{i} \quad|i-j| \geq 2 \\
\sigma_{i} \sigma_{i+1} \sigma_{i} & =\sigma_{i+1} \sigma_{i} \sigma_{i+1}
\end{array}
$$



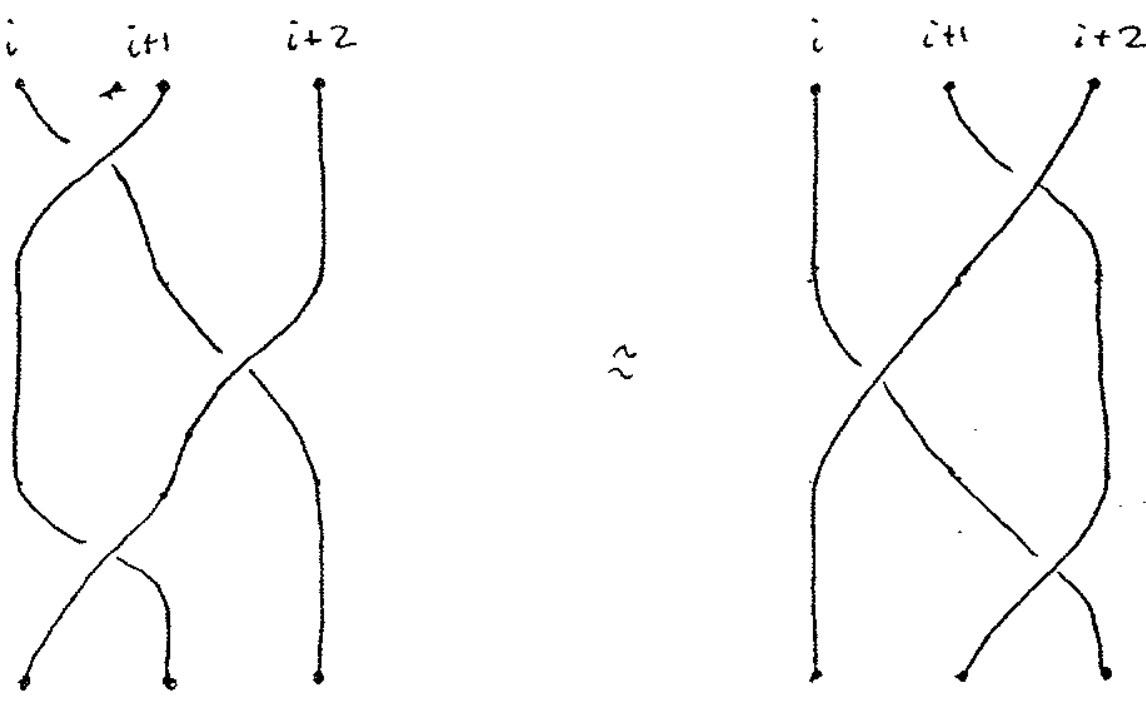

Fig. 2. Graphical representation of the relation $\sigma_{i} \sigma_{i+1} \sigma_{i}=\sigma_{i+1} \sigma_{i} \sigma_{i+1}$.

By looking at Fig. 1 it is fairly obvious that the first relation in (2.5a) is satisfied. The second is shown diagramatically in Fig. 2.

A basis in the space of blocks is equivalent to giving a skeleton representation of the blocks. In Fig. 3 one can see different bases for the 5-point function. The dimension of the space of blocks with fixed states on the external legs can be computed in terms of the fusion rules. The simplest information contained in the structure constants $C_{i j}^{k}$ is the number of possible couplings between the representations $i, j, k: N_{i j}^{k}$. The integral matrices $\left(N_{i}\right)_{j}^{k}=N_{i j}^{k}$ define the fusion algebra and later we will review some of its very striking properties ${ }^{15}$. Using $N_{i j}^{k}$ we can easily count the number of blocks. Take for example the 4-point block in (2.53), then the number of blocks with $k, l, n, m$ fixed is

$$
\begin{aligned}
\sum_{p} N_{l k}^{p} N_{n p}^{m} & =\left(N_{l} N_{n}\right)_{k}^{m} \\
\left(N_{i}\right)_{j}^{k} & \equiv N_{i j}^{k}
\end{aligned}
$$

it is clear that $N_{i j}^{k}=N_{j i}^{k}$ and furthermore associativity of the OPE implies (see Sec. 5) $\left[N_{i}, N_{j}\right]=0$; and the dimension of the space of blocks is the same regardless of the basis chosen (a consistency condition). We can write
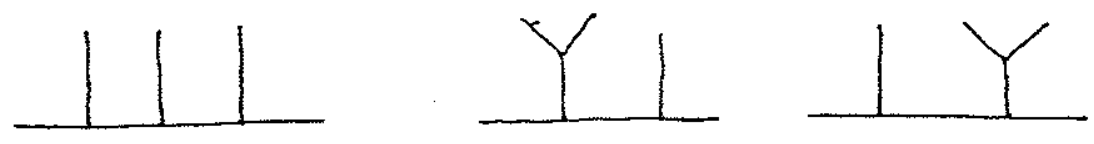

Fig. 3. Different skeletous represent different bases for the space of conformal blocks.

the associativity of the OPE and the locality of the correlation functions in terms of conformal blocks. There are two basic moves that one can make on the space of blocks. One correponds to the action of braid generator on the blocks and the other is a change of basis going from the s-channel to the $t$-channel. Following Moore and Seiberg ${ }^{16}$ we can call these moves respectively $B$-moves and $F$-moves (for braiding and fusing), and they are shown in Fig. 4.
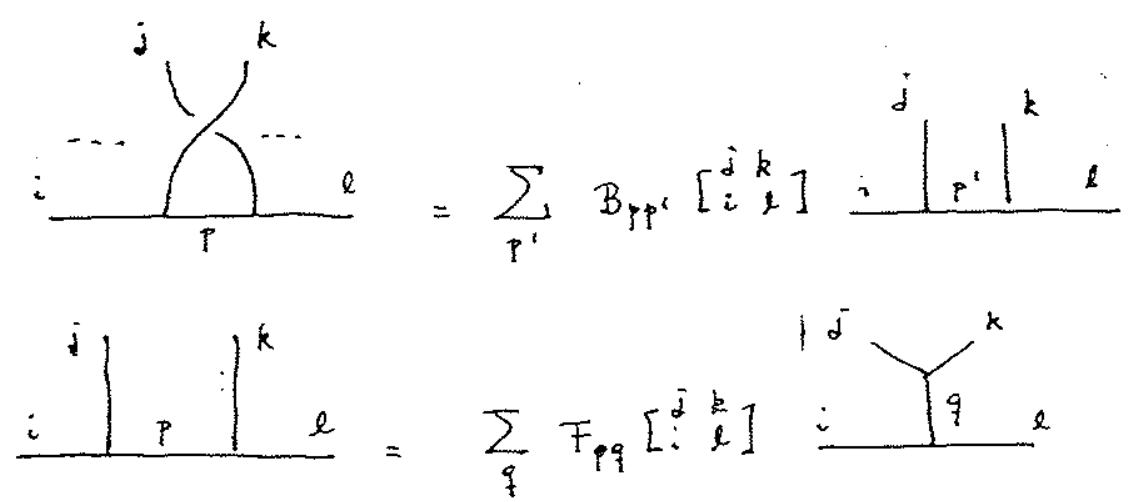

Fig. 4. The two basic moves in the space of blocks.

The first relation in Fig. 4 simply means that the conformal blocks provide representations of the braid group, and the second is the basic move that relates different bases in the space of blocks. Furthermore for CFT with a nonsingular scalar product the space of blocks provides a unitary representation of the braid group. We can now write a set of equations for $F$ and $B$ following from associativity of the $\mathrm{OPE}$ and the defining relations of the braid group. These two equations are the hexagon and pentagon identities of Moore and Seibeg ${ }^{16,17,18}$. They are explained graphically, in Figs. 5 and 6 . The two equations are not independent, but before we explain this it is convenient at this moment to study in more detail, the braid group 

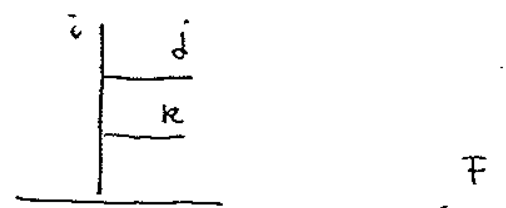

$$
F \uparrow
$$
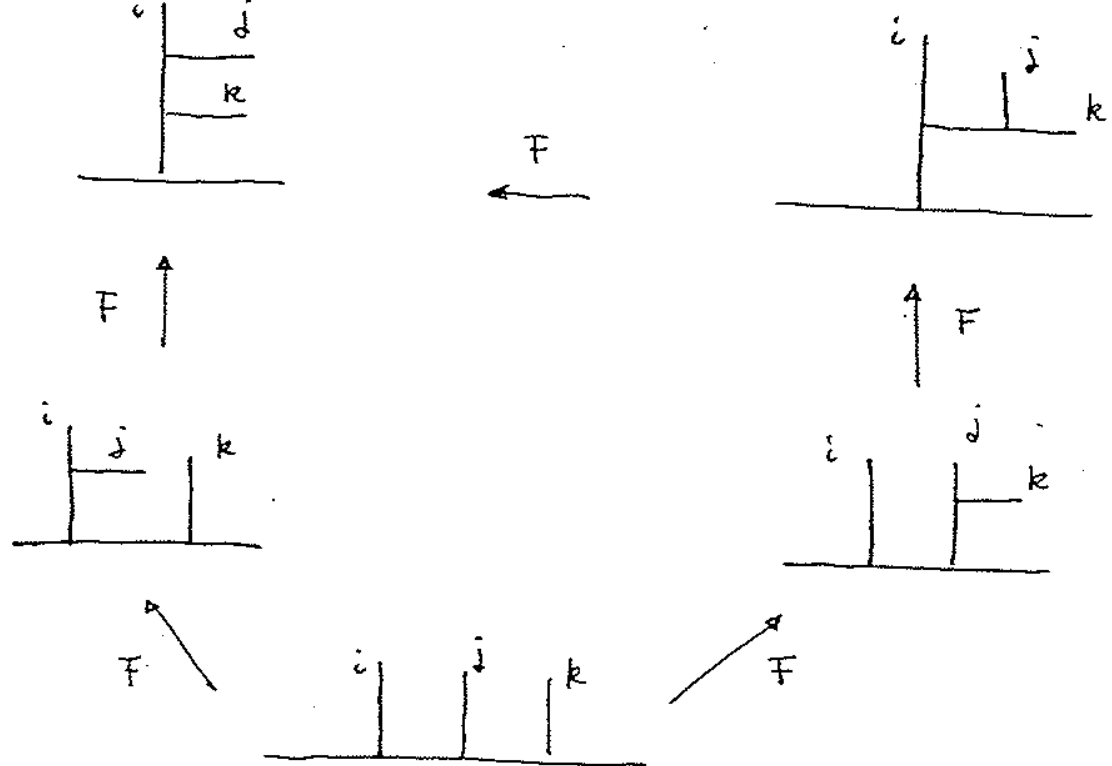

Fig. 5. Pentagon identity for $F$.

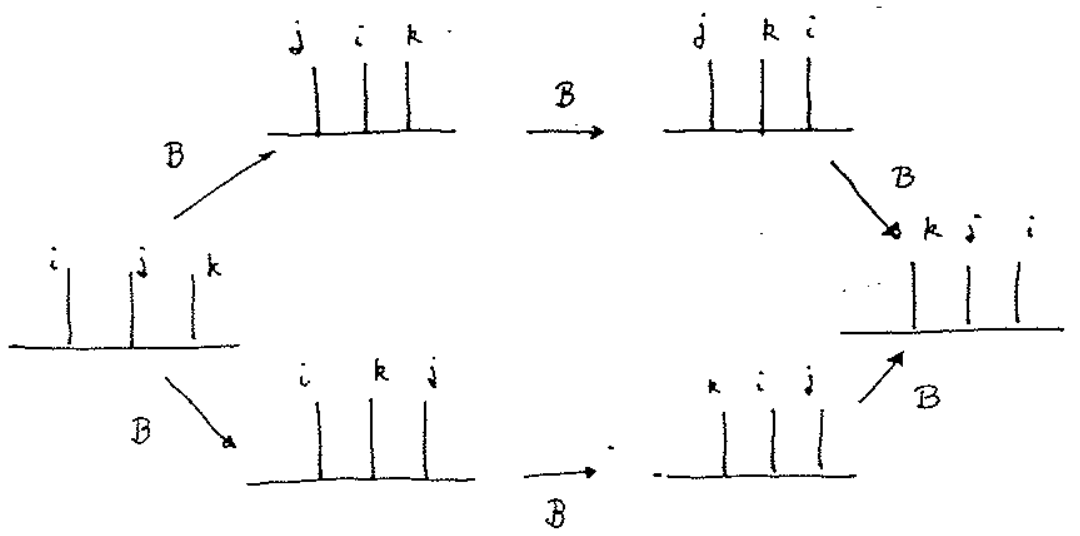

Fig. 6. Hexagon identity for B.

on the sphere and its relation to the mapping class group of the sphere with $n$ distinguished points and local parameters about them.

On the Euclidean plane $E^{2}$, the braid group $B_{n}\left(E^{2}\right)$ is completely defined by the relations (2.59). In fact we can construct all the generators in terms of two elements only: $\sigma_{1}, a=\sigma_{3} \ldots \sigma_{n-1}$. this is because

$$
\begin{aligned}
a \sigma_{1} & =\sigma_{1} \sigma_{2} \sigma_{3} \ldots \sigma_{n-1} \sigma_{1}=\sigma_{1} \sigma_{2} \sigma_{1} \sigma_{3} \ldots \sigma_{n-1} \\
& =\sigma_{2} \sigma_{1} \sigma_{2} \ldots \sigma_{n-1}=\sigma_{2} a \\
a \sigma_{2} \quad & =\sigma_{3} a \\
\ldots \ldots & \ldots \ldots \ldots \\
a \sigma_{n-2} & =\sigma_{n-1} a
\end{aligned}
$$

the center of $B_{n}\left(E^{2}\right)$ is generated by $a^{n}$ and it is an infinite cyclic group:

$$
a^{n} \sigma_{i}=\sigma_{i} a^{n} \quad i=1, \ldots, n-1
$$

The braid group on the sphere $B_{n}\left(S^{2}\right)$ is generated again by $\sigma_{1}, \ldots$ ,$\sigma_{n-1}$ satisfying (2.59) together with the relations:

$$
\begin{aligned}
& \sigma_{1} \sigma_{2} \ldots \sigma_{n-2} \sigma_{n-1}^{2} \sigma_{n-2} \ldots \sigma_{2} \sigma_{1}=1 \\
& \left(\sigma_{1} \sigma_{2} \ldots \sigma_{n-1}\right)^{n}=1
\end{aligned}
$$

These relations hold as long as the distinguished points do not come with local parameters. In CFT however we always have a local parameter about each point and we have to consider Dehn twist around the distinguished points. These twists are illustrated in Fig. 7 and they are represented in the CFT by the operator

$$
e^{2 \pi i\left(L_{0}-\bar{L}_{0}\right)}
$$

$e^{2 \pi i L_{0}}\left(e^{-2 \pi L_{0}}\right)$ acting on the holomorphic (aniholomorphic) part of the theory. Hence the effect of the twist on a purely left-(right-) moving state is:

$$
\begin{aligned}
& |h\rangle \rightarrow e^{2 \pi i h}|h\rangle \\
& |\bar{h}\rangle \rightarrow e^{-2 \pi i \bar{h}}|\bar{h}\rangle
\end{aligned}
$$

and this implies that in CFT on the sphere (or any other Riemann surface) with marked points and local parameters we do not obtain representations 


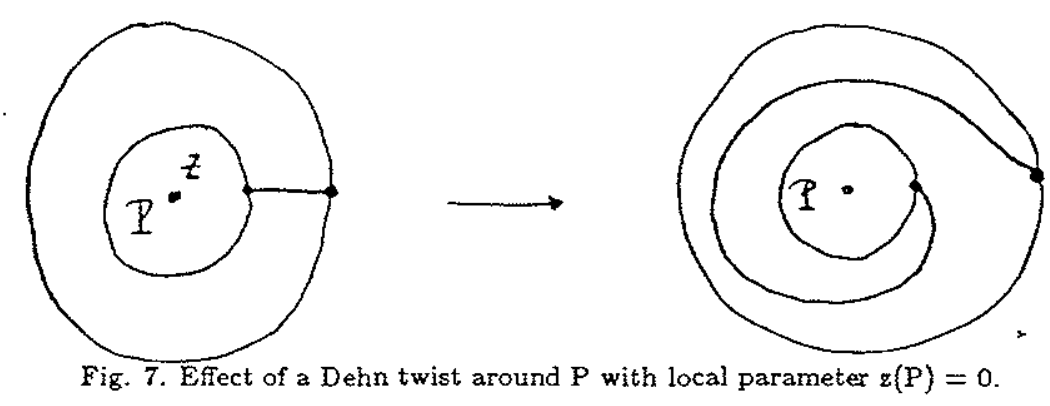

of $B_{n}\left(S^{2}\right)$ but rather of its extension by the Dehn twists around each punture.

There are several ways of finding the modification of the relations (2.62) due to Dehn twists. The easiest one is to consider the explicit form of the n-point functions (2.41) due to $\mathrm{SL}_{2}$ invariance ${ }^{22}$. When the local parameter is included one can think of braiding "ribbons" rather than braids, thus an operation which ends in the identity for braids may end up with twisted ribbons. Braiding the points $i$ and $j$ is expressed analytically as $z_{i j} \rightarrow e^{i \pi} z_{j i}$. Hence:

$$
z_{i j}^{-\gamma_{i j}} \rightarrow e^{-i \pi \gamma_{i, j}} z_{j i}^{-\gamma_{i j}}
$$

Hence the braiding of (2.41) will have two contributions: one coming from $\mathrm{SL}_{2}$ invariant quantities and the other from the prefactor. The first contribution gives a faithful representation of the braid group. If we perform the braiding operation $\sigma_{1} \ldots \sigma_{n-2} \sigma_{n-1}^{2} \sigma_{n-2} \ldots \sigma_{1}$ and consider the phase generated by the prefactor we obtain:

$$
\begin{aligned}
& e^{-i \pi \gamma_{12}} e^{-i \pi \gamma_{13}} \ldots e^{-i \pi \gamma_{1 n}} e^{-i \pi \gamma_{n 1}} e^{-i \pi \gamma_{n-1}} \ldots e^{-i \gamma_{11}} \\
& =\exp -2 \pi i \sum_{j \neq 1} \gamma_{1 j}=e^{-4 \pi i h_{1}} .
\end{aligned}
$$

Next, braiding according to $\left(\sigma_{1} \ldots \sigma_{n-1}\right)^{n}$ we obtain a phase:

$$
\begin{aligned}
& e^{-i \pi \gamma_{12}} \ldots e^{-i \pi \gamma_{1 n}} \\
& e^{-i \pi \gamma_{23}} e^{-i \pi \gamma_{34}} \ldots e^{-i \gamma_{2 n}} e^{-\pi \gamma_{21}} \\
& e^{-i \pi \gamma_{n 2}} e^{-i \pi \gamma_{n 3}} \ldots e^{-i \pi \gamma_{n n}-1}=\prod_{i} e^{-2 \pi i h_{i}}
\end{aligned}
$$

Therefore if $R\left(\sigma_{i}\right)$ is the representation of $\sigma_{i}$ on blocks we conclude:

$$
\begin{aligned}
& R\left(\sigma_{1} \mid \ldots R\left(\sigma_{n-2}\right) R\left(\sigma_{n-1}^{2}\right) R\left(\sigma_{n-2}\right) \ldots R\left(\sigma_{1}\right)=e^{-4 \pi i \hbar_{1}}\right. \\
& \left(R\left(\sigma_{1}\right) R\left(\sigma_{2}\right) \ldots R\left(\sigma_{n-1}\right)^{n}=\prod_{i=1}^{n} e^{-2 \pi i h_{i}}\right.
\end{aligned}
$$

where $h_{i}$ is the $L_{0}$ eigenvalue of the field in the ith strand. Since the dimensions of fields in the same conformal family differ by integers, the relations (2.68) hold regardless of which representatives (primary or descendants) we choose in each conformal family. This is a general consequence of the fact that the braiding properties depend on the conformal families and not on their representatives (exercise: prove it).

The mapping clase group of the sphere with $n$ distinguished points is closely related to the braid group ${ }^{7}(2.59)$ and (2.62). It is generated by Dehn twists around pairs of points and single points. Given two points $P_{i}, P_{j+1}$ the Dehn twist is constructed by considering a simple closed curve $C$ enclosing $P_{i}, P_{j}$ and no other distinguished points. We cut the surface along $C$, twist one of the lips by $2 \pi$ and glue back again. Its action on blocks can be constructed in terms of $R\left(\sigma_{i}\right)$. If $h_{i}, h_{i+1}$ and the dimensions of the fields at the points $i, i+1$ it follows from the definition that the Dehn twist $\tau_{i, i+1}$ about $P_{i}, P_{i+1}$ is represented by:

$$
\tau_{i, i+1}=e^{2 \pi i\left(h_{i}+h_{i+1}\right)} R\left(\sigma_{i}^{2}\right)
$$

and any other twist can be obtained from these ones and twists about single points. If we consider the particular case of the sphere with 4 points, the generators of the mapping class group are $\tau_{12}, \tau_{13}, \tau_{23}, \tau_{1}, \tau_{2}, \tau_{3}, \tau_{4}$ and using (2.68) and (2.69) one can show:

$$
\tau_{12} \tau_{13} \tau_{23}=\tau_{1} \tau_{2} \tau_{3} \tau_{4}
$$

This relation has been used by $\mathrm{Vafa}^{19}$ to show that for theories with a finite number of primary fields (rational CFT), the dimensions $h_{i}$ are all rational numbers.

To exhibit the connection between the matrices $B$ and $F$, we notice that if we braid the $i, j$ legs in Fig. 8 , we obtain:

$$
R\left(\sigma_{i j}\right) W_{k}^{i j}=e^{i \pi\left(h_{k}-h_{i}-h_{j}\right)} W_{k}^{j i}
$$




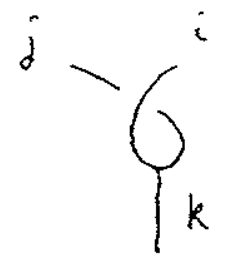

Fig. 8. Braiding in the three point block
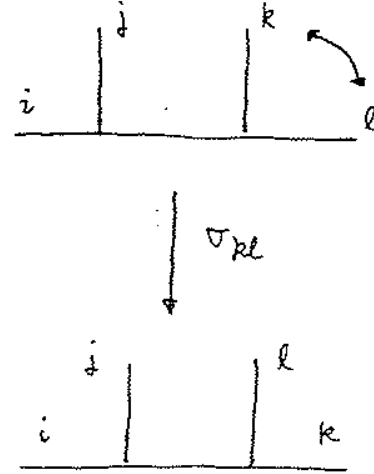
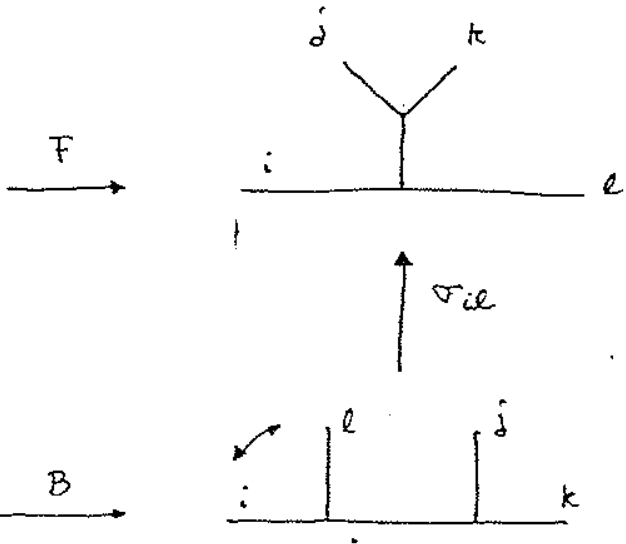

Fig. 9. Relation between $F$ and $B$ matrices.

only a phase appears. If we now consider the set of moves in Fig. 9 , it is easy to see from (2.71) that $B$ and $F$ are related by phases.

To conclude, the duality (crossing) properties of a CFT can be rewritten in terms of blocks using the $B$ and $F$ matrices. The content of the duality conditions reduces to the pentagon or hexagon identities plus the $\sigma$-move (2.71). These equations are the tree level polynomial equations. ${ }^{16,18}$ If the CFT has only a finite number of primary fields one says that the theory is a Rational Conformal Field Theory (RCFT), and the hexagon and pentagon identities becomes a fnite set of conditions. When one includes the constraints arising from genus one modular invariance one obtains the complete set of equations characterizing the duality properties of a RCFT ${ }^{16,18}$. The classification of RCFT can be phased in part as the solution of these polynomial equations. A difficult (and interesting) open problem is to what extent a RCFT can be reconstructed from a solution to these equations. After building more machinery we will analyze this question in more detail. We have reviewed quickly the general constraints of duality at the level of conformal blocks to set the framework for futher discussion. Later, in
Sec. 5 we will study in more details, the fusion algebra and the rationality constraints imposed by (2.68) on the set of conformal dimensions.

\section{Degenerate Conformal Families. Coulomb Gas Repretation \\ 3.1 Generalities}

In this subsection we present general properties of conformal theories with null vectors. In the next subsection we will study an explicit formulation of these theories in terms of a coulomb gas representation of the conformal blocks. This will allow us to prove (or at least make plausible) some of the general results. In particular we will derive the representation of conformal blocks in terms of contour integrals, the fusion rules for the minimal conformal theories ${ }^{6,8}$, the Rocha-Caridi ${ }^{10}$ formulae for the Virasoro characters and a few sample calculations of monodromy invariants and structure constants of the operator algebra.

To understand the meaning of null vectors, we go back to ordinary group theory. When we study the finite dimensional representations of simple or semisimple groups we are accustomed to a general property of the representation theory of this group which implies that any reducible representation can always be brought to block diagonal form i.e. reducible representations are fully reducible. This however is not true in general. To assess whether this is the case we need to use the Braver-Weyl theory which follows from Schur's lemma. Given an irreducible representation $V$ of some algebra $A$ (a Lie algebra for example) we can take arbitrary tensor products $V \otimes V \otimes \ldots \otimes V=V^{\otimes n}$. If $A$ is the Lie algebra of $\mathrm{SU}(N)$ and $V$ is the fundamental representation, we can decompare $V^{\otimes n}$ into irreducible representations labelled by Young tableaux defining different symmetries for a tensor of rank $n$. The steps in these decompositions are as follows: We first construct the centralizer algebra $B$ of the action of $A$ on $V^{\otimes n}$. In other words, we look for all operators on $V^{\otimes n}$ which commute with the representation of $A$ in $V^{\otimes n}$. For $S U(N)$ the centralizer in $V^{\otimes n}$ is the permutation group $S_{n}$ exchanging the different copies of $V$ in $V \otimes n$ if $n \leq N$. When $n>N$ we have $S_{n}$ with the extra constraint that the $N+1$ row antisymmetrizer vanishes; there are no Young tableaux with more than $N$ rows. The question of complete reducibility of $V^{\otimes n}$ reduces then to the question of whether the centralizer algebra is semi-simple. For the case of $\mathrm{SU}(N)$ this is the case as well as for other semi-simple groups. When this is not the case one finds representation in $V^{\otimes n}$ which generically contains null vectors (i.e. more than one highest weight state) which are not fully 
reducible. We will find some finite dimensional examples when we come to study the representation theory of quantum groups.

What is the analogous situation for the Virasoro algebra? We have seen in the previous section that the braiding properties of the conformal blocks are independent of the representative fields chosen for each conformal family on the block. Since changing representatives in each family is equivalent to acting with the Virasoro algebra on the primary fields, we find that the centralizer of the Virasoro action on a collection of conformal blocks contains at least the braid group (for RCFT this should be the whole thing). If we find null vectors in the Verma modules associated with our CFT this spells trouble in the representations of the duality transformations acting on the conformal blocks. Thus, to obtain a sensible theory (with a nondegenerate scalar product which guarantees that the space of blocks provides a fully reducible representation of the duality and braid transformations) we have to eliminate the null vectors and consider the reduced theory. ${ }^{a}$ This leads to a collection of nontrivial differential equations for the blocks which in some cases completely determine the correlation functions of the theory. To be more precise, let $V(c, h)$ be a Verma module of the Virasoro algebra Vir, with highest weight vector $|h\rangle, L_{0}|h\rangle=h|h\rangle$, then:

$$
L_{0}\left(L_{-n_{2}} \ldots L_{-n_{N}}|h\rangle\right)=\left(h+\sum_{i} n_{i}\right)\left(L_{-n_{1}} \ldots L_{-n_{N}}|h\rangle\right)
$$

the integer $\sum n_{i}$ is the level of the state and $|h\rangle$ has zero level. If the representation is unitary, $h>0$,

$$
\| L_{-1}|h\rangle \|^{2}=\left\langle h\left|L_{+1} L_{-1}\right| h\right\rangle=2 h>0 .
$$

Furthermore by taking the $2 \times 2$ matrix of scalar products of $L_{-N}|h\rangle$ and $L_{-1}^{N}|h\rangle$ and requiring it to be positive we can obtain that $c>0$. If we can find a state $|\chi\rangle \in V(c, h)$ such that:

$$
\begin{aligned}
& \left.L_{0}|\chi=(h+N)| \chi\right\rangle \\
& L_{n}|\chi\rangle=0 \quad n>0
\end{aligned}
$$

${ }^{a}$ When we study the fusion algebra in more detail (Sec. 5) we will see that for sensible physical theories the space of blocks should provide a fuily reducible representation of the braid group. we say that $|\chi\rangle$ is a null vector and the representation is degenerate. If we have a scalar product such that $L_{n}^{+}=L_{-n}$, then one shows that $\langle\psi \mid \chi\rangle=0$ for any state of the theory. Hence we can set $|\chi\rangle=0$ which is equivalent to taking the quotient by the null vectors and the submodules they generate. If $\phi_{h}(z)$ is the primary field generating the family we say that the family is degenerate and that it has a null vector at level $N$. As an example we consider null vectors a level two:

$$
\left(a L_{-2}+b L_{1}^{2}\right)|h\rangle
$$

since all the $L_{n} n>0$ are generated by $L_{1}$ and $L_{2}$ we only need to impose the annihilation of (3.4) by $L_{1}$ and $L_{2}$ to obtain the conditions for a null vector. This gives a constraint for given $c$ of what modules can have a leve two null state. We obtain:

$$
\begin{aligned}
& |\chi\rangle=\left(L_{-2}-\frac{3}{2(2 h+1)} L_{-1}^{2}\right)|h\rangle \\
& h=\frac{1}{16}(5-c \pm \sqrt{(c-1)(c-25)}) .
\end{aligned}
$$

Requiring the decoupling of $|\chi\rangle$ from every correlation function leads to a second order differential equation for correlation functions containing $\phi_{h}(z)$. If $\chi_{h}(z)$ is the descendant field creating (3.5), we have:

$$
\begin{aligned}
& \left\langle\chi_{h}(z) \phi_{1}\left(z_{1}\right) \ldots \phi_{N}\left(z_{N}\right)\right\rangle \\
= & \left(\frac{3}{2(2 h+1)} \frac{\partial^{2}}{\partial z^{2}}-\sum_{i=1}^{N} \frac{h_{i}}{\left(z-z_{i}\right)^{2}}-\sum_{i=1}^{N} \frac{1}{z-z_{i}} \frac{\partial}{\partial z_{i}}\right)\left\langle\phi_{h}(z) \phi_{1} \ldots \phi_{N}\right\rangle=\underset{(3.6)}{0}
\end{aligned}
$$

where (2.36) and (2.37) have been used. For the 4-point function, using $\mathrm{SL}_{2}$ invariance this leads to a hypergeometric differential equation whose solutions will be given later in terms of contour integrals. V.Kac has given the set of representations of Vir with null vectors. To obtain this set one has to consider for a given $V(c, h)$ and any level $N$ the determinant of the scalar products between all the states at level $N$. The zeroes of this Kac determinant give the values of $(c, h)$ of representations with a level $N$ null vector. To write the result in a simple form, we introduce two numbers $\alpha_{+} \alpha_{-}$defined by:

$$
\begin{aligned}
& c=1-24 \alpha_{0}^{2} \\
& \alpha_{0}=\alpha_{+}+\alpha_{-}, \alpha_{+} \alpha_{-}=-1 .
\end{aligned}
$$


Then the representations with null vectors depend on two integers $n, m \geq 1$ and have dimensions:

$$
h(n, m)=-\frac{1}{4} \alpha_{0}^{2}+\frac{1}{4}\left(n \alpha_{+}+m \alpha_{-}\right)^{2}
$$

and $V_{n, m}=V\left(c, h_{n, m}\right)$ has a null vector at level $n m$. This fact has a simple derivation in the Coulomb gas representation.

The null vectors also give a lot of information about the fusion rules of the theory ${ }^{6}$. To exhibit how this is obtained, we consider $h=h(1,2)$ or $h(2,1)$. In this case the null vector appears at level 2 , and the differential equation obtained by decoupling thern is (3.6). If $\phi_{1}\left(z_{1}\right)$ has dimension $h_{1}$ and we take $z \sim z_{1}$, we have the OPE:

$$
\phi_{h}(z) \phi_{h_{1}}\left(z_{1}\right)=\text { coust. }\left(z-z_{1}\right)^{k}\left[\phi_{h 1}\left(z_{1}\right)+b\left(z-z_{1}\right) \phi_{h 1}^{(-1)}\left(z_{1}\right)+\ldots\right]
$$

the expansion (3.9) solves (3.6) if

$$
\frac{3}{2(2 h+1)} k(k-1)-h_{1}+k=0
$$

and scale invariance implies

$$
k=h^{\prime}-h_{1}-h .
$$

Parametrizing dimensions as:

$$
h_{1}=-\frac{1}{4} \alpha_{0}^{2}+\frac{1}{4} \alpha^{2}, h^{\prime}=-\frac{1}{4} \alpha_{0}^{2}+\frac{1}{4} \alpha^{2}
$$

we find

$$
\begin{array}{ll}
h=h(1,2) & \alpha^{\prime}=\alpha \pm \alpha_{-} \\
h=h(2,1) & \alpha^{\prime}=\alpha \pm \alpha_{+}
\end{array}
$$

giving the fusion rules

$$
\begin{aligned}
& \phi_{(1.2)} \phi_{(\alpha)}=\left[\phi_{\left(\alpha_{-} \alpha_{-}\right)}\right]+\left[\phi_{\left(\alpha_{+} \alpha_{-}\right)}\right] \\
& \phi_{(2,1)} \phi_{(\alpha)}=\left[\phi_{\left(\alpha_{-} \alpha_{+}\right)}\right]+\left[\phi_{\left(\alpha_{+} \alpha_{+}\right)}\right] .
\end{aligned}
$$

The normalization however remains to be determined. Depending on the theory some of the families on the right-hand side may not appear.
Similar arguments can be used to derive the general fusion rules once the null vectors are known explicitly. With the information we have, we can explore a little the operator algebra of degenerate theories. The simplest fusion rules we can write down are:

$$
\begin{aligned}
& \phi_{(1,2)} \phi_{(1,2)}=\left[\phi_{(1,1)}\right]+\left[\phi_{(1,3)}\right] \\
& \phi_{(2,1)} \phi_{(2,1)}=\left[\phi_{(1,1)}\right]+\left[\phi_{(3,1)}\right]
\end{aligned}
$$

the field $\phi_{(1,1)}$ has zero dimension and it is identified as the identity operator. The normalization requires the associativity of the OPE and some properties of the theory under consideration. Applying (3.13) once more we find:

$$
\begin{aligned}
& \phi_{(1,2)} \phi_{(1, m)}=\left[\phi_{(1, m-1)}\right]+\left[\phi_{(1, m+1)}\right] \\
& \phi_{(2,1)} \phi_{(m, 1)}=\left[\phi_{(m-1,1)}\right]+\left[\phi_{(m+1,1)}\right] .
\end{aligned}
$$

Thus if $\phi_{(1,2)}$ is on the operator algebra so is $\phi_{(1, m)}$ generically and the same for $\phi_{(2,1)}$ and $\phi_{(m, 1)}$. Naive application of these rules would however generate dimension $h(n, m)$ with $(n, m)$ zero or negative. In fact these fields drop from the operator algebra, and only $\phi_{(n, m)} n, m>0$ appear. This truncation phenomenon can be understood as follows:

$$
\phi_{(1,2)} \phi_{(2,1)}=c_{1}\left[\phi_{(2,0)}\right]+c_{2}\left[\phi_{(2,2)}\right] .
$$

Using the second relation in (3.13) we have:

$$
\phi_{(1,2)} \phi_{(2,1)}=c_{1}^{\prime}\left[\phi_{(0,2)}\right\}+c_{2}^{\prime}\left\{\phi_{(2,2)}\right\}
$$

and the $c$ and $c^{\prime}$ are the OPE coefficients. Compatibility between (3.16) and (3.17) requires $c_{1}=c_{1}^{\prime}=0, c_{2}=c_{2}^{\prime}$, and therefore:

$$
\phi_{(1,2)} \phi_{(2,1)}=\left[\phi_{(2,2)}\right] \text {. }
$$

It is clear that if $\phi_{(1,2)}$ and $\phi_{(2,1)}$ are in the operator algebra we can generate all the filds $\phi_{(n, m)}$ in the theory via OPE. Moreover, using the truncation phenomenon and the associativity of the OPE we can derive the fusion rules for a theory with degenerate fields $\phi_{(n, m)}$. The result is ${ }^{6}$

$$
\phi_{\left(n_{1}, m_{3}\right)} \phi_{\left(n_{3}, m_{2}\right)}=\sum_{k=\left(n_{1}-n_{3}\right)+1}^{n_{1}+n_{3}-1} \sum_{l=\left(m_{1}-m_{2}\right)+1}^{m_{1}+m_{2}-1}\left[\phi_{(k, l)}\right] .
$$


If $\left|n_{1}-n_{2}\right|$ is odd (even) then the sum over $k$ runs only over even (odd) values, and similarly for $\left|m_{1}-m_{2}\right|$ and $l$.

Next one can try to find theories where all the field are degenerate and with a finite number of fields (RCFT). From (3.7) and (3.8) we see that when $c>25 \alpha_{+}, \alpha_{-}$are purely imaginary, and as $n, m$ grow larger some $h(n, m)$ will become negative. For $25>c>1$, the dimensions are generally complex. Only for $c \leq 1$ the dimensions are real in general. For example when $c=1, \alpha_{0}=0, \alpha_{ \pm}= \pm 1$, and the representations with null vectors are those such that $h=N^{2} / 4, N$ an integer. When $\alpha_{-} / \alpha_{+}$is a rational number it was shown in Ref. 6 that the operator algebra truncates to a finite set. This is because each module now contains an infinite number of null fields (we will show this later in Sec. 3.2. If $p, q$ are relatively prime positive integers, then the minimal model of Belavin Polyakov and Zamolodchikov satisfy:

$$
\begin{aligned}
& \alpha_{-} / \alpha_{+}=-p / q \quad c=1-\frac{6(p-q)^{2}}{p q} \\
& h(n, m)=\frac{1}{4 p q}\left[(n q-m p)^{2}-(p-q)\right)^{2}
\end{aligned}
$$

The unitary subseries ${ }^{8}$ is obtained for $q=p+1$ and $p \geq 2$

$$
c=1-\frac{6}{p(p+1)}, L_{n, m}=\frac{1}{4 p(p+1)}\left[(n(p+1)-m p)^{2}-1\right]
$$

Notice furthermore the reflection property:

$$
h(n, m)=h(p-n, q-m)
$$

and we can restrict the $n, m$ values to the rectangle:

$$
0<n<p \quad 0<m<q \quad, p<q
$$

The simplest nontrivial example is the Ising model with $p=3, q=4$. Now $c=1 / 2$ and we have a grid of conformal dimensions shown in Fig. 10, given by:

$$
\begin{aligned}
& h(1,1)=h(2,3)=0 \\
& h(1,2)=h(2,2)=\frac{1}{16} \\
& h(2,1)=h(1,3)=\frac{1}{2}
\end{aligned}
$$

$\phi_{(1,1)}$ (or $\left.\phi_{(2,3)}\right)$ is identified with the identity operator, $\phi_{(1,2)}$ (or $\phi_{(2,2)}$ ) is the spin field $\sigma$ and $\phi_{(2,1)}\left(\phi_{(1,3)}\right)$ is identified with the energy density $\varepsilon$. The fusion rules are easy to derive:

$$
\begin{aligned}
& \varepsilon \cdot \varepsilon=\phi_{(2,1)} \times \phi_{(2,1)}=c_{1}\left[\phi_{(1,1)}\right]+c_{2}\left[\phi_{(3,1)}\right] \\
& =\phi_{(1,3)} \times \phi_{(1,3)}=c_{1}^{\prime}\left[\phi_{(1,1)}\right]+c_{2}^{\prime}\left[\phi_{(1,3)}\right]+c_{3}^{\prime}\left[\phi_{(1,5)}\right] \text {. } \\
& {\left[\begin{array}{cccc}
(2,1) & (2,2) & (2,3) & \vdots \\
\bullet & \ddots & \ddots & \vdots \\
& & & \vdots \\
(1,1) & (1,2) & (1,3) & \vdots
\end{array}\right.}
\end{aligned}
$$

Fig. 10. Conformal weight for $p=3, q=4$.

Hence $c_{2}=c_{2}^{\prime}=c_{3}^{\prime}=0$, and $\varepsilon \cdot \varepsilon=[1]$. Similarly for the other operator products. We obtain:

$$
\begin{gathered}
\varepsilon \times \varepsilon=[1] \\
\varepsilon \times \sigma=[\sigma] \quad \sigma \times \sigma=[1]+[\varepsilon]
\end{gathered}
$$

and similar arguments apply to other cases. This concludes the generalities of degenerate theories. Next we find explicit representations for their correlation functions and characters.

\subsection{Coulomb gas representation. Fusion rules}

The properties of all the minimal models can be obtained in terms of single scalar field $l$. We follow in part the presentation in Ref. 9. Consider the following energy-momentum tensor:

$$
\begin{aligned}
& T(z)=-\frac{1}{4} \partial \phi(z) \partial \phi(z)+i \alpha_{0} \partial^{2} \phi(z) \\
& \langle\phi(z) \phi(w)\rangle=-2 \ln (z-w) \\
& c=1-24 \alpha_{0}^{2} .
\end{aligned}
$$

$T(z)$ follows from the variation of the action of a free scalar field with some background charge: a term propartional to $R \phi$ in the Lagrangian, 
- where $R$ is the two-dimensional scalar curvature. The background charge is $e=-2 \alpha_{0}$. Hence the $\mathrm{U}(1)$ current $\partial \phi(z)$ is anomalous:

$$
T(z) \partial \phi(w)=\frac{1}{(z-w)^{2}} \partial \phi(w)+\frac{1}{z-w} \partial^{2} \phi(w)+\frac{4 i \alpha_{0}}{(z-w)^{3}} .
$$

The last term represents the anomaly. The primary fields are exponentials of $\phi$, and the background charge changes the conformal dimension with respect to the $\alpha_{0}=0$ case:

$$
T(z): e^{i \alpha \phi(w)}:=\frac{\alpha\left(\alpha-2 \alpha_{0}\right)}{(z-w)^{2}}: e^{i \alpha \phi(w)}+\frac{1}{z-w} \partial_{w} e^{i \alpha \phi(w)}+\text { regular }(3.28)
$$

hence

$$
h\left(: e^{i \alpha \phi}:\right)=\alpha\left(\alpha-2 \alpha_{0}\right)
$$

and the correlation functions of these vertex operators will vanish unless the background charge is screened:

$$
\begin{aligned}
\left\langle V_{\alpha_{1}}\left(z_{1}\right) \ldots V_{\alpha_{n}}\left(z_{n}\right)\right\rangle & =\prod_{i<j}\left(z_{i}-z_{j}\right)^{+2 \alpha_{i} \alpha_{j}} \quad \text { if } \sum \alpha_{i}=2 \alpha_{0} \\
& =0 \text { otherwise } \\
V_{\alpha}(z) & =: e^{i \alpha \phi(z)}: .
\end{aligned}
$$

From (3.29) we learn that there are always two fields of dimension 1 :

$$
\begin{aligned}
& J_{ \pm} \quad=: e^{i \alpha_{ \pm} \phi}: \\
& \alpha_{+}+\alpha_{-m}=2 \alpha_{0}, \quad \alpha_{+} \alpha_{-}=-1 .
\end{aligned}
$$

This justifies a posteriori the definitions in (3.7). We want to use this construction to represent the primary fields and correlation functions of the minimal models. It is important to notice that both $V_{\alpha}(z)$ and $V_{2 \alpha_{0}-\alpha}(z)$ have the same dimension. In particular we can write the identity in two ways, 1 and $\exp 2 i \alpha_{0} \phi:$. The existence of currents of dimension one allows us to introduce screening operators $Q_{ \pm}$:

$$
Q_{ \pm}=\oint_{c} J_{ \pm}(z) d z
$$

Inserting $Q_{ \pm}$in correlation functions does not change the conformal properties. If we write down the 4-point function of an operator $V_{\alpha}(z)$.

$$
\left\langle V_{\alpha} V_{\alpha} V_{\alpha} V_{\check{\alpha}}\right\rangle
$$

the total charge is $3 \alpha+2 \alpha_{0}-\alpha=2 \alpha+2 \alpha_{0}$; hence will vanish generically unless:

$$
2 \alpha_{0}=-n \alpha_{+}-m \alpha_{-}
$$

in which case we can introduce $n Q_{+}$and $m Q_{-}$screening operators to obtain a nonvanishing amplitude. Therefore the spectrum of vertex operators with nonvanishing 4-point functions (3.33) is given by

$$
\alpha_{n, m}=\frac{1-n}{2} \alpha_{+}+\frac{1-m}{2} \alpha_{-} \quad, n, m \geq 1
$$

and using (3.24) their dimensions are:

$$
h(n, m)=-\frac{1}{4} \alpha_{0}^{2}+\frac{1}{4}\left(n \alpha_{+}+m \alpha_{-}\right)^{2} .
$$

Equations (3.35) and (3.36) reproduce the Kac spectrum of degenerate conformal families. Hence a 4-point block will take the form:

$$
\begin{aligned}
& \oint_{c_{1}} d t_{1} \ldots \oint_{c_{N}} d t_{N} \oint_{c_{1}^{\prime}} d t_{1}^{\prime} \ldots \oint_{c_{m}^{\prime}} d t_{m}^{\prime}\left\langle V_{\alpha^{\prime}}\left(z_{1}\right) V_{\alpha_{2}}\left(z_{2}\right) V_{\alpha_{3}}\left(z_{3}\right) V_{\alpha_{1}}\left(z_{4}\right)\right. \\
& \left.J_{+}\left(t_{1}\right) \ldots J_{+}\left(t_{N}\right) J_{-}\left(t_{1}^{\prime}\right) \ldots J_{-}\left(t_{M}^{\prime}\right)\right\rangle_{-2 \alpha_{0}}
\end{aligned}
$$

where the correlation function is evaluated in terms of a free scalar field with background charge $-2 \alpha_{0}$ (from now on we will remove the subindex $-2 \alpha_{0}$ from $<\ldots>$ ).

As a first application of this construction we derive the fusion rules (3.19). We want to find the fields $\phi_{(k, l)}$ appearing in the OPE of $\phi_{(m, n)}$ and $\phi_{(r, s)}$. To do this we only need to concentrate on the three-point function. In the Coulomb gas representation, and using $\mathrm{Sl}_{2}$ invariance, there are three equivalent ways of representing the three point function:

$$
\begin{aligned}
& \left\langle V_{\overline{(k, l)}}(\infty) V_{(m, n)}(1) V_{(r, o)}(0) Q_{+} Q_{-}\right\rangle \\
& \left\langle V_{(k, l)}(\infty) V_{\overline{(m, n)}}(1) V_{(r, o)}(0) Q_{+} Q_{-}\right\rangle \\
& \left\langle V_{(k, l)}(\infty) V_{(m, n)}(1) V_{\overline{r, o}}(0) Q_{+} Q_{+}\right\rangle
\end{aligned}
$$

(the exponents in $Q_{ \pm}$are not indicated because we will not need them. It suffices to know that one can always screen the charge of the three vertex operators). From (3.35) and (3.38a), the correlation function will not vanish if:

$$
\begin{aligned}
& k \leq m+r-1 \text { with } k-m-r-1 \text { even } \\
& l \leq n+s-1 \text { with } l-n-s-1 \text { even }
\end{aligned}
$$


and similarly with (3.38b) and (3.38c), we thus obtain:

$$
\left.\left.\begin{array}{l}
k \leq m+r-1 \\
m \leq r+k-1 \\
r \leq k+m-1
\end{array}\right\} \quad k+m+r \quad \text { odd } \begin{array}{r}
n \leq n+s-1 \\
s \leq l+n-1
\end{array}\right\} \quad l+n+s \quad \text { odd (3.41) }
$$

which implies:

$$
\phi_{(m, n)} \times \phi_{(r, s)}=\sum_{\substack{k=|m-r|+1 \\ k+m+n=\text { odd }}}^{m+r-1} \sum_{\substack{l=|n-s|+1 \\ l+n+s=\text { odd }}}^{n+s-1}[\phi(k, l)] .
$$

We now consider the minimal series (3.20). We know that $h(n, m)=h(p-$ $n, q-m)$, then:

$$
\begin{aligned}
& \phi_{(n, m)} \times \phi_{(r, s)}=\sum I_{a=|n-r|+1}^{n+r-1} \sum \sum I_{b=|m-s|+1}^{m+s-1}\left[\phi_{(a, b)}\right]
\end{aligned}
$$

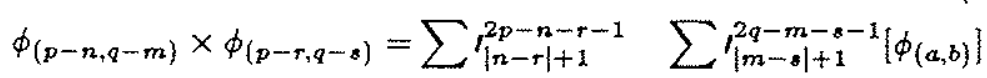

where $\sum^{\prime}$ means that we sum with constraint $k+m+n=0$ etc. (as in (3.41)). Equations (3.42a) and (3.42b) are compatible as long as:

$$
\phi_{(n, m)} \times \phi_{(r, a)}=\sum_{|n-r|+1}^{\min (n+r-1,2 p-1-n-r)}
$$

giving the fusion rules for the minimal models. Setting $q=p+1$ we obtain the fusion rules for the unitary series ${ }^{8}$. As we will see in the next section these fusion rules are analogous to the fusion rules for $S U(2)_{p-2} \times S U(2)_{q-2}$ Wess-Zumino-Witten models ${ }^{11,12,13}$.

\subsection{Virasoro characters and null vectors}

Before turning into the computation of correlation functions, we can write down the Virasoro characters of the minimal models ${ }^{10}$ as a simple application of the Kac formula. This will give us a chance to exhibit the tower of null states in each Verma module appearing in the minimal series. Given a representation of the Virasoro algebra $V$ with highest weight vector $h$ and central extension $c$, the character of this representation is defined by:

$$
\begin{aligned}
\chi_{V}(q) & =q^{h-c / 24} \sum_{N=0}^{\infty} a(N) q^{N} \\
& =\operatorname{tr} V q^{L_{0}-c / 24}
\end{aligned}
$$

where $N$ is the level, and $a(N)$ is the number of states at level $N$. If there are no null vectors, a basis of $V$ is given by

$$
V=\left\{L_{-n_{1}} \ldots L_{-n_{N}}|h\rangle n_{1}>0\right\}
$$

obtained by applying any polynomial in the $L_{-n} n>0$ to the highest weight state. Since $\left[L_{0}, L_{-n}\right]=n L_{-n}$ the character of $V$ is easily seen to be:

$$
\chi(q)=\frac{q^{h-c / 24}}{\prod_{n=1}^{\infty}\left(1-q^{n}\right)}
$$

when there are null vectors we have to subiract the submodules generated by the null vectors in order to obtain the character of the irreducible representation associated to a given primary field in the conformal theory.

Take $p, q$ to be relatively prime integers. The Verma module $V_{(n, m)}$ obtained as in (3.45) will have plenty of null vectors for $h=h_{(n, m)}(3.20)$. In fact according to $\mathrm{Kac}$ formula there is a null vector at level $n m$ with $L_{0}$ eigenvalve $h(n, m)+n m=h(n,-m)$. Some formulae useful for the next arguments are:

$$
\begin{aligned}
& h(n, m)=\frac{1}{4 p q}\left[(n q-m p)^{2}-(p-q)^{2}\right] \\
& h(n, m)=h(p-n, q-m)=h(-n,-m)=h(p+n, q+n) \\
& a(k) \quad \equiv h(n+2 p k, m)=h(n, m-2 p k) \\
& b(k) \quad \equiv h(n+2 p k,-m)=h(n,-m-2 q k) .
\end{aligned}
$$

If the highest weight vector is $h(n, m)=a(0)$, there are two null vectors

1) $h(n, m)+n m=h(n,-m)=b(0)$

2) $h(p-n, q-m)+(p-n)(q-m)=h(p-n, m-q)=h(n, 2 q-m)=b(-1)$ 
the two null vectors have dimensions $b(0), b(-1)$. If we compute the naive character $(3.46)$ for $V_{(n, m)}$, then we have to subtract the contribution of the two null submodules:

$$
\frac{q^{-c / 24}}{\prod_{n=1}^{\infty}\left(1-q^{n}\right)}\left(q^{a(0)}-q^{b(0)}-q^{b(-1)}\right) .
$$

This is not the whole story however. The modules with highest weights $b(0), b(-1)$ overlap and have themselves null vectors, so we are not doing the correct subtraction in (3.48). To get a complete picture of the null vector pattern we consider the general case. There are four possibilities:

1. A module with highest weight vector $a(k)=h(n+2 p k, m)=$ $h(n, m-2 q k)=h(q-n, q+2 q k-m), k>0$. When $k>0 ; q-n>$ $0, q+2 q k-m>0$, then $h(n+2 p k, m)$ has a null vector of dimension $h(n+2 p k,-m)=b(k)$. There is a second null vector which is obtained by writing $a(k)$ as $h(p-n, q+2 k q-m)$, and it has dimension $h(p-n, m-$ $q-2 q k)=h(p-n, q+m-2 q(k+1))=h(n,-m+2 q(k+1))=b(-k-1)$. Hence $a(k), k>0$ has two null submodules with highest weight vectors of dimensions $b(k)$ and $b(-k-1)$.

2. Next take $a(-k), k>0$. Then:

$$
a(-k)=h(n-2 p k, m)=h(n, m+2 q k)=h(p+2 p k-n, q-m) \text {. }
$$

Since $p+2 p k-n>0, q-m>0$, we are back into Kac formula, and there is a null vector of dimension

$$
\begin{aligned}
h(p+2 p k-n, m-q) & =h(2 p(k+1)-n, m)=h(n-2 p(k+1),-m) \\
& =b(-k-1) .
\end{aligned}
$$

Writing now $a(-k)=h(n, m+2 q k)$ we learn again from Kac formula that there is a second null vector with dimension $h(n,-m-2 q k)=b(k)$. Hence $a(-k), k>0$ has two null submodules with highest weight dimensions $b(k), b(-k-1)$

3. A module with highest weight $h=b(k) k>0$. Then

$$
b(k)=h(n+2 p k,-m)=h(n+2 p k+p, q-m) .
$$

Both entries are positive integers, and by Kac formula there is a null vector of dimension

$$
h(n+2 p k+p, m-q)=h(n+2 p(k+1), m)=a(k+1) .
$$

Furthermore:

$$
b(k)=h(n+2 p k,-m)=h(n,-m-2 q k)=h(p-n, 2 q k+q+m) .
$$

In the both term both entries are positive, and there is a second null vector with dimension:

$$
h(p-n,-2-q k-q-m)=h(n, 2 q(k+1)+m)=a(-k-1) .
$$

Thus $V_{b(k)}$ has two null submodules with dimensions $a(k+1), a(-k-1)$.

4. Finally we have $b(-k), k>0$. Then

$$
b(-k)=h(n-2 p k,-m)=h(2 p k+p-n, q+m)=h(2 p k-n, m)
$$

with a null vector $h(n-2 p k, m)=a(-k)$. Similarly: $b(-k)=h(n-$ $2 p k,-m)=h(n,-m+2 q k)$, and there is a second null vector at $h(n, m-$ $2 q k)=a(k)$

We represent all the cases $1-4$ in terms of all embedding diagram of modules in Fig. 11. The quantities $a(k), b(k)$ label the Verma modules $V_{a(k)}, V_{b(k)}$ by the $L_{0}$ eigenvales of their highest weight vectors.

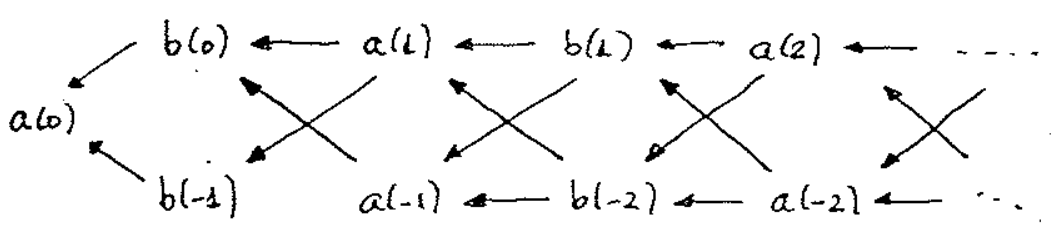

Fig. 11. Null vector structure of a Verma module in the minimal series

This diagram allows us to correct for the over (or under) subtraction of null states in (3.48). A simple conbinatorial argument now yield the formula for the Virasoro characters given by Rocha-Caridi ${ }^{10}$ :

$$
\begin{aligned}
& \chi_{n, m}(q)=\frac{q^{-c / 24}}{\prod_{l=1}^{\infty}\left(1-q^{d}\right)} \sum_{k \in \mathbb{Z}}\left(q^{a(k)}-q^{b(k)}\right) \\
& a(k) \equiv h(n+2 p k, m)=\frac{(2 p q k+q n-m p)^{2}-(p-q)^{2}}{4 p q} \\
& b(k) \equiv h(n+2 p k,-m)=\frac{(2 p q k+q n+m p)^{2}-(p-q)^{2}}{4 p q} .
\end{aligned}
$$


These characters generate a lot of interesting identities. For instance Euler's pentagonal number theorem is obtained if we take $p=2, q=3$. Then we have $c=0$, and only the identity operator, hence $\chi_{(1,1)}=1$ :

$$
\begin{aligned}
& \chi_{(1,1)}=1=\frac{1}{\prod_{l=1}^{\infty}\left(1-q^{l}\right)} \sum_{k \in \mathbb{Z}}\left(q^{6 k^{2}+k}-q^{6 k^{2}+5 k+1}\right) \\
& \prod_{l=1}^{\infty}\left(l-q^{l}\right)=\sum_{k \in \boldsymbol{Z}}(-1)^{k} q^{\left(3 k^{2}+k\right) / 2} .
\end{aligned}
$$

Another example is the character for the spin representation in the Ising model: $p=3, q=4, c=1 / 2, h_{1,1}=0, h_{(1,2)}=h_{(2,2)}=1 / 16$. Then:

$\chi_{1 / 16}(q)=\frac{q^{1 / 16}}{\prod_{n=1}^{\infty}\left(1-q^{n}\right)} \sum_{k}\left(q^{12 k^{2}+2 k}-q^{12 k^{2}+10 k+2}\right)=q^{1 / 16} \prod_{n=1}^{\infty}\left(1+q^{n}\right)$

where the last equality is obtained from the known equivalence between the critical Ising model and a free Weyl-Majorana fermion.

In the character computation just completed we only needed to konw the conformal dimension of the highest weight states in the null submodules. If one wants to explicitly verify that null vectors decouple from correlation functions, we can represent the null vectors explicitly in the Coulomb gas formulation. The naive Verma module (3.45) in this formulation is obtained by first expanding the field $\phi$ in oscillators. If $V_{h}(z)$ is the vertex operator creating the highest weight state of dimension $h ;|h\rangle, V_{h}$ is generated by acting on $|h\rangle$ with arbitrary polynomials in the creation operators contained in $\phi$. The oscillator representation of the null vectors was given by Fateev and Zamolodchikov ${ }^{43}$. We will need the relations: $\alpha_{n, m}$

$2 \alpha_{0}-\alpha_{n, m}$

$$
=\frac{1-n}{2} \alpha_{+}+\frac{1-m}{2} \alpha_{-}
$$$$
2 \alpha_{0}-\alpha_{n, m}-n \alpha_{0}
$$$$
=\frac{1+n}{2} \alpha
$$$$
=\alpha_{n,-m}
$$$$
+\frac{1+m}{2} \alpha_{-}=\alpha_{-n,-m}
$$

$2 \alpha_{0}-\alpha_{n, m}-m \alpha_{-}=\alpha_{-n, m}$ $\alpha_{n, m}+n \alpha_{+}=\beta_{-n, m}$

$\alpha_{n, m}+m \alpha_{-}=\beta_{n,-m}$, then it is not difficult to show that

$$
\begin{aligned}
& \chi_{n m}^{+}=\oint_{c_{1}} \ldots \oint_{c_{n}} J_{+}\left(z_{1}\right) \ldots J_{+}\left(z_{n}\right) V_{\substack{\alpha(n,-m) \\
\alpha(+n,-m)}}(z) \\
& \chi_{n, m}^{-}=\oint_{c_{1}} \ldots \oint_{c_{m}} J_{-}\left(z_{1}\right) \ldots J_{-}\left(z_{m}\right) V_{\substack{\alpha(-n, m) \\
\alpha(-n,+m)}}(z)
\end{aligned}
$$

are respectively null vectors of $V_{2 \alpha_{0}}-\alpha_{n, m}$. The proof is simple. From standard vertex operator manipulations:

$$
\begin{aligned}
J_{+}\left(z_{1}\right) \ldots J_{+}\left(z_{n}\right) V_{\alpha(n,-m)}(0)= & \prod_{j<j}\left(z_{i}-z_{j}\right)^{2 \alpha_{+}^{2}} \prod_{i} z_{i}^{2 \alpha+\alpha(n,-m)} . \\
& : J_{+}\left(z_{1}\right) \ldots J_{+}\left(z_{n}\right) V_{\alpha(n,-m)}(0): \\
{\left[L_{n}, J_{ \pm}(z)\right]=\partial_{z}\left(z^{n+1} J_{ \pm}(z)\right) . } &
\end{aligned}
$$

If we choose the contours $C_{1}, C_{2}, \ldots$ so that there is no cut, we obtain:

$$
L_{k} \chi_{n, m}=Q_{+}^{n} L_{k} V_{\alpha(n,-m)}(0)+\oint d z^{\prime} s \partial_{z_{1}}\left(z_{1}^{k+1} \ldots\right)+\ldots
$$

and the contour integrals on the right-hand side vanish. Hence

$$
L_{k} \chi_{n, m}=Q_{+}^{n} L_{k} V_{\alpha(n, m)}(0)
$$

and:

$$
\begin{aligned}
& L_{k} \chi_{n, m}=0 \quad k>0 \\
& L_{0} \chi_{n, m}=\left(h_{n, m}+n m\right) \chi_{n, m}
\end{aligned}
$$

Let us consisder an example with $n=2$ :

$$
\oint_{c_{1}} \oint_{c_{2}} d z_{1} d z_{2}\left(z_{1}-z_{2}\right)^{2 \alpha_{+}^{2}}\left(z_{1} z_{2}\right)^{2 \alpha_{+} \alpha_{(\lambda,-m)}}: J_{+}\left(z_{1}\right) J_{+}\left(z_{2}\right) V_{\alpha(n,-m)}(0):
$$

From (3.52) and changing the variables to $z_{1}=z, z_{2}=z \xi(3.57)$ becomes:

$$
\oint_{c_{z}} d z \oint_{c_{\xi}} d \xi z^{-1-2 m} \xi^{-\alpha_{+}^{2}-(1+m)}(1-\xi)^{2 \alpha_{+}^{2}} .: J_{+}(z) J_{+}(\xi z) V_{n,-m}(0): \text {. }
$$




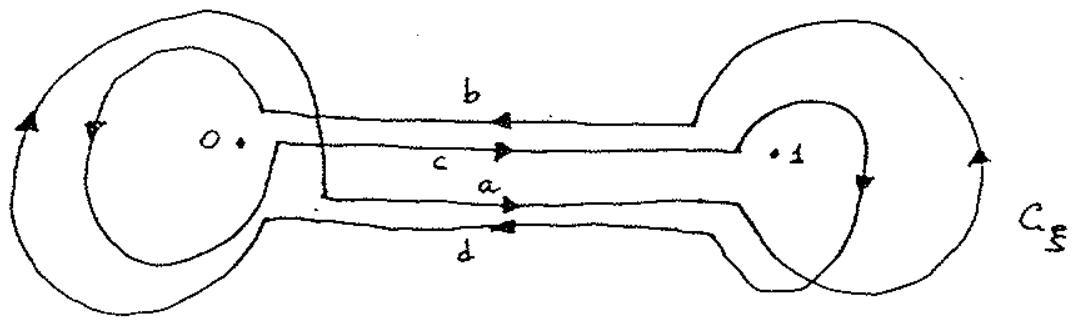

Fig. 12. Integration contour in (3.59).

The $z$ integral will bring down some powers of $\xi$ and some oscillator operators and the coefficients are given by integrals of the form:

$$
\oint_{c_{\xi}} d \xi \xi^{-\alpha_{+}^{3}-(1+m)+N}(1-\xi)^{2 \alpha_{+}^{2}}, 0 \leq N \leq n m
$$

Equation (3.59) is evalvated with the contour in Fig. 12.

When we go around this contour, the integrand comes back to the same value. Keeping track of the phase differences between $a, b, c, d$ we obtain:

$$
\begin{aligned}
I(\alpha, \beta) & =\oint_{c_{\xi}} \xi^{\alpha-1}(1-\xi)^{\beta-1} \\
& =\left(1-e^{2 \pi i \alpha}\right)\left(1-e^{2 \pi i \beta}\right) \oint_{0}^{1} d \xi \xi^{\alpha-1}(1-\xi)^{\beta-1} \\
& =-4 e^{i \pi(\alpha+\beta)} \sin \pi \alpha \sin \pi \beta \frac{\Gamma(\alpha) \Gamma(\beta)}{\Gamma(\alpha+\beta)} \\
\alpha & =-\alpha_{+}^{2}-m+N, \beta=2 \alpha_{+}^{2}+1 .
\end{aligned}
$$

The integrand has four sheets and two branch points, and the genus of the surface is zero by the Riemann-Hurwitz theorem. For more complicated examples the genus of the Riemann surface defined by the integrand will generically be higher than zero.

Using the representation (3.53) for null vectors it is also possible to derive the Kac spectrum of dimensions. Finally a simple consequence of these contour manipulation is that

$$
\begin{aligned}
& Q_{+}^{n} V_{\alpha_{(n, m)}}(0)=0 \\
& Q_{-}^{m} V_{\alpha_{(n, m)}}(0)=0 .
\end{aligned}
$$

Before we considered the module $V_{\left(2 \alpha_{0} \cdots \alpha_{n, m}\right)}=V_{\alpha_{-n,-m}}$, and found a null vector with the vertex operator $V_{\alpha_{(n,-m)}}$. Now consider the module $V_{\left(\alpha_{(-n, m)}\right)}$. In the multiple contour integral

$$
\oint_{c_{1}} \ldots \oint_{c_{n}} J_{+}\left(z_{1}\right) \ldots J_{+}\left(z_{n}\right) V_{\alpha_{n, m}}(0)
$$

we use (3.54) and make the change of variables $z_{i}=z_{1} \xi_{i}, i=2, \ldots, n ; \xi_{1}=$ 1. Using (3.52) we see that the $z_{1}$ integral is well-defined. The power of $z_{1}$ in the integrand is $z_{1}^{-1+n m}$, hence $Q_{+}^{n} V_{\alpha_{n, m}}(0)$ is a null vector in the module $V_{\left(\alpha_{-n, m}\right)}$. Since the highest weight vector of this module has $h=h_{n, m}+n m$ and $Q_{+}^{n} V_{\alpha_{n, m}}(0)$ has $h=h_{n, m}, Q_{+}^{n} V_{\alpha_{n, m}}(0)$ must necessarily vanish. A similar argument works for $Q_{-}^{m} V_{\alpha_{n, m}}(0)$. The general procedure described up till now is quite useful in the construction of the Kac formula, characters and null vectors for algebras other than Virasoro admitting a Coulomb gas representation.

\subsection{Sample computations: con formal blocks and braiding matrices}

We now come to the explicit computation of correlation functions and fusion and braiding matrices for the minimal theories in this representation ${ }^{9}$ : For simplicity we consider correlation functions containing a single screening operator. For instance:

$$
\left\langle\phi_{(\overline{(n, m)})} \phi_{(1,2)} \phi_{(1,2)} \phi_{(n, m)}\right\rangle
$$

has charge $2 \alpha_{0}-\alpha_{-}$, and therefore it requires one $Q_{-}$, and

$$
\left\langle\phi_{(n, m)} \phi_{(2,1)} \phi_{(2,1)} \phi_{(n, m)}\right\rangle
$$

has charge $2 \alpha_{0}-\alpha_{+}$and it requires one power of $Q_{+}$. From the free field two-point function (3.26) we obtain:

$$
\begin{gathered}
\oint d t\left\langle V_{\alpha_{1}}\left(z_{1}\right) V_{\alpha_{3}}\left(z_{2}\right) V_{\alpha_{3}}\left(z_{3}\right) V_{\alpha_{4}}\left(z_{4}\right) J_{ \pm}(t)\right\rangle \\
=\left(\prod_{i<j} z_{i j}^{2 \alpha_{i} \alpha_{j}}\right) I\left(z_{i}\right) \\
I\left(z_{i}\right)=\oint_{d t} \prod_{i}\left(z_{i}-t\right)^{2 \alpha_{i} \alpha_{ \pm}} .
\end{gathered}
$$


We can write this block in a more connonical form using $\mathrm{SL}_{2}$ invariance

$$
\begin{aligned}
\left\langle\prod_{i} V_{i}\left(z_{i}\right) J_{ \pm}(t)\right\rangle= & \prod_{i=1}^{4}\left(c z_{i}+d\right)^{-2 h_{i}}(c t+d)^{-2} \\
& \left\langle\prod V_{i}\left(\frac{a z_{i}+b}{c z_{i}+d}\right) J_{ \pm}\left(\frac{a t+b}{c t+d}\right)\right\rangle .
\end{aligned}
$$

Since $t$ is integrated and $J_{ \pm}$is a 1 -form we can forget about the $t$-transformation. We now choose $a, b, c, d$ so that $z_{1} \rightarrow \infty, z_{2} \rightarrow 1, z_{3} \rightarrow \eta, z_{4} \rightarrow 0$ where

$$
\eta=\frac{z_{12} z_{34}}{z_{13} z_{24}}
$$

This is achieved by:

$$
w=\frac{\left(z-z_{4}\right)\left(z_{1}-z_{2}\right)}{\left(z_{1}-z\right)\left(z_{2}-z_{4}\right)}
$$

leading to:

$$
\begin{aligned}
& \oint d t\left\langle\prod_{i} V_{i}\left(z_{i}\right) J_{ \pm}(t)\right\rangle=\left(\frac{z_{12} z_{14}}{z_{24}}\right)^{h_{2}+h_{3}+h_{4}-h_{1}} \\
& \frac{(1-\eta)^{2 \alpha_{2} \alpha_{3}} \eta^{2 \alpha_{3} \alpha_{4}}}{z_{12}^{2 h_{2}} z_{13}^{2 h_{3}} z_{14}^{2 h_{4}}} \oint_{c} d t(1-t)^{2 \alpha_{2} \alpha_{ \pm}}(\eta-t)^{2 \alpha_{3} \alpha_{ \pm}} t^{2 \alpha_{4} \alpha_{ \pm}} .
\end{aligned}
$$

The integral can be evalvated in terms of hypergeometric functions:

$$
F(a, b, c ; z)=\frac{\Gamma(c)}{\Gamma(b) \Gamma(c-b)} \oint_{0}^{1} t^{b-1}(1-t)^{c-b-1}(1-t z)^{-a} d t
$$

and we will need the properties:

$$
\begin{aligned}
& F(a, b, c ; z)=(1-z)^{c-a-b} F(c-a, c-b, c ; z) \\
& F(-n, n, c ; z)=\frac{1}{(c)_{n}} z^{1-c}(1-z)^{n-b+c} \frac{d^{n}}{d z^{n}}\left[z^{n+c-1}(1-z)^{b-c}\right] \\
& \quad(c)_{n}=c(c+1) \ldots(c+n-1) \\
& \cos a z=\cos z F\left(\frac{1}{2}+\frac{a}{2}, \frac{1}{2}-\frac{a}{2}, \frac{1}{2} ; \sin ^{2} z\right) \\
& \sin a z=a \cos z \sin z F\left(1+\frac{a}{2}, 1-\frac{a}{2}, \frac{3}{2} ; \sin ^{2} z\right)
\end{aligned}
$$

In (3.65) there are two possible contours of integration shown in Fig. 13. Notice that we are drawing $c_{1}, c_{2}$ as open contours. This requires some

explanation. Generically $0, \eta, 1, \infty$ will be branch points of the integrand in (3.65), then the open contour integral and the closed contour integral enclosing a cut differ only by a normalization factor. Since the normalization of the blocks is fixed by the convertion (2.54), we need not worry at this moment about the overall normalization of the integral in (3.65). The difference is quite important however in correlation function. This is very useful, because it gives a different procedure to compute the fusion rules. If the exponenets in the integrand (3.65) conspire in such a way that one of the 2 points in Fig. 13 is not a branch point, then one of the closed contour integrals will vanish. The possible intermediate states in the block (3.62) are determined by the integration contours, hence when one contour gives a vanishing contribution we obtain information about which operators appear in the OPE of the operators in the external legs. Summarizing, even though the contours in Fig. 13 are drawn as open contours, for purpose of determining the fusion rules they should be understood as closed contours on the Riemann surface defined by the integrand.

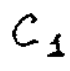

1

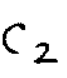

$C_{2}$

$$
\text { o }
$$

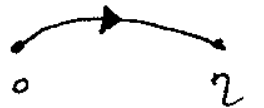

1

$\infty$

As sample computations we look at the 4-point blocks of the Ising model. We have eight blocks to compute (many of them are related by monodromy as we will see presently):

$$
\begin{aligned}
& \langle\varepsilon \varepsilon \varepsilon \varepsilon\rangle\langle\varepsilon \varepsilon \sigma \sigma\rangle\langle\varepsilon \sigma \epsilon \sigma\rangle\langle\varepsilon \sigma \sigma \varepsilon\rangle \\
& \langle\sigma \varepsilon \varepsilon \sigma\rangle\langle\sigma \sigma \varepsilon \varepsilon\rangle\langle\sigma \varepsilon \sigma \varepsilon\rangle\langle\sigma \sigma \sigma \sigma\rangle
\end{aligned}
$$

for the Ising model:

$$
\begin{aligned}
& \varepsilon=e^{i \alpha_{2,1} \phi} \quad c=\frac{1}{2}, p=3, q=4 \\
& \sigma=e^{i \alpha_{1,2} \phi} \quad h_{2,1}=\frac{1}{2}, \alpha_{2,1}=-\alpha_{+} / 2
\end{aligned}
$$




$$
h_{1,2}=\frac{1}{16} \alpha_{1,2}=-\alpha_{-} / 2
$$

Using (3.65) and (3.66), the integrals along the $c_{1}, c_{2}$ contours needed are:

$$
\begin{aligned}
& \int_{0}^{2} d t_{\left(c_{1}\right)}(1-t)^{\alpha}(\eta-t)^{\beta} t^{\gamma}= \frac{\Gamma(1+\gamma) \Gamma(1+\beta)}{\Gamma(2+\gamma+\beta)} \eta^{1+\beta+\gamma} \\
& F(-\alpha, 1+\gamma, 2+\gamma+\beta ; \eta) \\
& \int_{1}^{\infty} d t(1-t)^{\alpha}(\eta-t)^{\beta} t^{\gamma}= \\
& \\
& \quad F(1+\alpha,-\alpha-\beta-\gamma-1,-\beta-\gamma ; \eta) .
\end{aligned}
$$

Start with $\langle\varepsilon \varepsilon \varepsilon \varepsilon\rangle$. The two contours give contributions:

$$
\langle\varepsilon \varepsilon \varepsilon \varepsilon\rangle=\frac{1}{z_{13} z_{24}}[\eta(1-\eta)]^{2 / 3} \begin{cases}{[\eta(1-\eta)]^{-5 / 3} F(-2,-1 / 3,-2 / 3 ; \eta)} & 1) \\ F(4 / 3,3,8 / 3 ; \eta) & 2)\end{cases}
$$

In the second contribution $t=0$ is not a branch point and therefore the second contour does not contribute. Using $(3.67 \mathrm{~b})$ we obtain:

$$
\langle\varepsilon \varepsilon \varepsilon \varepsilon\rangle=\frac{1}{z_{13} z_{24}} \frac{1-\eta+\eta^{2}}{\eta(1-\eta)}
$$

satisfying the normalization condition (2.54) and implying that the intermediate state is the identity. Hence the fusion rule is $\varepsilon \times \varepsilon=1$. In terms of blocks we can write (3.72) as:

$$
\langle\varepsilon \varepsilon \varepsilon \varepsilon\rangle=\frac{\left.||^{\varepsilon\left(z_{2}\right)}\right|^{\varepsilon\left(z_{3}\right)}}{\varepsilon\left(z_{1}\right)} \circ \underbrace{}_{\left(z_{4}\right)}=F_{0}^{\epsilon \kappa \kappa \epsilon}\left(z_{i}\right)=\frac{1-\eta+\eta^{2}}{z_{13} z_{24} \eta(1-\eta)} .
$$

Proceding analogously with all other blocks in (3.68) we obtain after some algebra:

$$
F_{0}^{\epsilon \varepsilon \varepsilon \epsilon}\left(z_{i}\right)=\frac{1-\eta+\eta^{2}}{z_{13} z_{24} \eta(1-\eta)}=\frac{\varepsilon||_{0}^{\varepsilon} \epsilon}{\varepsilon}
$$

$$
\begin{aligned}
& F_{0}^{\text {Topics in Conformal Field Theory }}\left(z_{i}\right)=\left(z_{13} z_{24}\right)^{-1 / 8} \frac{(1-\eta)^{3 / 8}}{\eta^{1 / 8} \sqrt{2}}\left[\frac{1+\sqrt{1-\eta}}{1-\eta}\right]^{1 / 2}=\frac{\left.\sigma\right|_{0} ^{\sigma} \sigma}{\sigma 5} \\
& F_{\epsilon}^{\sigma \sigma \sigma \sigma}=\left(z_{13} z_{24}\right)^{-1 / 8} \sqrt{2} \frac{(1-\eta)^{3 / 8}}{\eta^{1 / 8}}\left[\frac{1-\sqrt{1-\eta} \eta}{1-\eta}\right]^{1 / 2}=\sigma|\varepsilon|^{\sigma} \sigma \\
& z_{0}^{\epsilon \epsilon \sigma \sigma}=\frac{(1-\eta)^{-1 / 2}}{z_{12} z_{34}^{1 / 8}}\left(1-\frac{1}{2} \eta\right) \\
& =\left.\varepsilon \underbrace{\varepsilon}\right|^{\sigma} \sigma \\
& z_{\sigma}^{\varepsilon \sigma \varepsilon \sigma}=\frac{1-2 \eta}{z_{13} z_{24^{1 / 8}}[\eta(1-\eta)]^{1 / 2}} \\
& =\varepsilon^{\sigma}|\sigma|^{\varepsilon} \sigma \\
& \mathcal{F}_{\sigma}^{E \sigma \sigma \varepsilon}=\frac{z_{14}}{\left(\frac{z_{13} z_{24}}{z^{1 / 8}} \frac{1}{z_{14}}\right.} \frac{1+\eta}{\eta^{1 / 2}(1-\eta)^{1 / 8}} \\
& =\varepsilon|\sigma| \varepsilon \\
& z_{0}^{\sigma \sigma \ll \epsilon}=\frac{1}{z_{12}^{1 / 8} z_{34}} \frac{1-\eta / 2}{(1-\eta)^{1 / 2}} \\
& =\sigma||_{0}^{\varepsilon} \varepsilon \\
& \mathcal{F}_{\sigma}^{\sigma \sigma \sigma \kappa}=\frac{[\eta(1-\eta)]^{1 / 2}}{z_{13}^{1 / 8} z_{24}}(1-2 \eta) \\
& =\sigma^{\varepsilon}|\sigma| \varepsilon \mid \varepsilon \\
& \xi_{\sigma}^{\sigma \varepsilon \varepsilon \sigma}=\frac{(1-\eta)^{-1} \eta^{-1 / 2}}{z_{14}^{1 / 8} z_{13} z_{24}} z_{14}(1+\eta) \\
& =\sigma^{\varepsilon}|\sigma| \varepsilon
\end{aligned}
$$


Taking the limit $z_{1}=\infty, z_{4}=0,(3.74)$ simplifies somewhat:

$$
\begin{aligned}
& \eta \quad=z_{3} / z_{2} \quad 1-\eta=z_{23} / z_{2}
\end{aligned}
$$

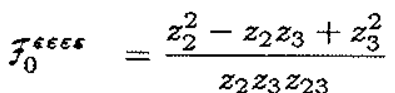

$$
\begin{aligned}
& F_{0}^{\sigma \sigma \sigma \sigma}=\frac{1}{\sqrt{2}}\left(z_{2} z-3 z_{23}\right)^{-1 / 8}\left(z_{2}^{1 / 2}+z_{23}^{i / 2}\right)^{1 / 2} \\
& f_{\varepsilon}^{\sigma \sigma \sigma \sigma}=\sqrt{2}\left(z_{2} z_{3} z_{23}\right)^{-1 / 8}\left(z_{2}^{1 / 2}-z_{23}^{1 / 2}\right)^{1 / 2} \\
& \xi_{0}^{\varepsilon \epsilon \sigma \sigma}=\frac{1}{z_{2}^{1 / 2} z_{23}^{1 / 2} z_{3}^{1 / 8}}\left(z_{2}-\frac{1}{2} z_{3}\right) \\
& \mathcal{f}_{\sigma}^{* \sigma \epsilon \sigma}=\frac{z_{2}-2 z_{3}}{z_{3}^{1 / 2} z_{23}^{1 / 2} z_{2}^{1 / 8}}
\end{aligned}
$$

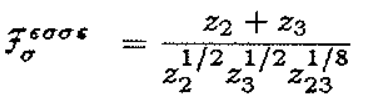

$$
\begin{aligned}
& \xi_{0}^{\sigma \sigma \epsilon \epsilon}=\frac{z_{2}-\frac{1}{2} z_{3}}{z_{2}^{1 / 2} z_{3} z_{23}^{1 / 2}} \\
& \xi_{\sigma}^{\sigma \sigma \sigma \varepsilon}=\frac{z_{-2} 2 z_{3}}{z_{3}^{1 / 2} z_{2} z_{23}^{1 / 2}} \\
& \mathcal{F}_{\sigma}^{\sigma \kappa * \sigma}=\frac{z_{2}+z_{3}}{z_{2}^{1 / 2} z_{3}^{1 / 2} z_{23}}
\end{aligned}
$$

and we can compute the braiding matrices $B$ in Fig. 4 . The braiding 2 and 3 is equivalent to the analytic continuation $z_{23} \rightarrow e^{i \pi} z_{32}$. For example

$$
\begin{aligned}
& \sigma_{2} \xi_{0}^{\epsilon \epsilon \varepsilon \varepsilon}(2,3)=e^{-i \pi} \mathcal{F}_{0}^{\epsilon \epsilon \varepsilon \varepsilon}(3,2)=B_{00}\left[\begin{array}{ll}
\varepsilon & \varepsilon \\
\varepsilon & \varepsilon
\end{array}\right] \mathcal{F}_{0}^{\epsilon \epsilon \varepsilon \varepsilon}(3,2) \\
& \sigma_{2} \mathcal{F}_{0}^{\epsilon \epsilon \sigma \sigma}(2,3)=-\frac{1}{2} \varepsilon^{-i \pi} \xi_{\sigma}^{\epsilon \sigma \varepsilon \sigma}(3,2) \quad B_{0 \sigma}\left[\begin{array}{ll}
\varepsilon & \sigma \\
\varepsilon & \sigma
\end{array}\right]=-\frac{1}{2} e^{-i \pi}(3.76)
\end{aligned}
$$

with only one possible internal state in the block, the computation is rather simple, and we obtain apart from (3.76): the only complicated case comes with braiding in the $\langle\sigma \sigma \sigma \sigma\rangle$ blocks. Using the identities:

$$
\begin{aligned}
{\left[y^{1 / 2}+i(x-y)^{1 / 2}\right]^{1 / 2}=} & \frac{e^{i \pi / 4}}{\sqrt{2}}\left[x^{1 / 2}+(x-y)^{1 / 2}\right]^{1 / 2}+ \\
& \frac{e^{-i \pi / 4}}{\sqrt{2}}\left[x^{1 / 2}-(x-y)^{1 / 2}\right]^{1 / 2} \\
{\left[y^{1 / 2}-i(x-y)^{1 / 2}\right]^{1 / 2}=} & \frac{e^{-i \pi / 4}}{\sqrt{2}}\left[x^{1 / 2}-(x-y)^{1 / 2}\right]^{1 / 2}+ \\
& \frac{e^{-i \pi / 4}}{\sqrt{2}}\left[x^{1 / 2}+(x-y)^{1 / 2}\right]^{1 / 2}
\end{aligned}
$$

we easily obtain:

$$
\left(\begin{array}{cc}
B_{00}\left[\begin{array}{ll}
\sigma & \sigma \\
\sigma & \sigma
\end{array}\right] & B_{0 \varsigma}\left[\begin{array}{ll}
\sigma & \sigma \\
\sigma & \sigma
\end{array}\right] \\
B_{\Delta 0}\left[\begin{array}{ll}
\sigma & \sigma \\
\sigma & \sigma
\end{array}\right] & B_{\varepsilon \epsilon}\left[\begin{array}{ll}
\sigma & \sigma \\
\sigma & \sigma
\end{array}\right]
\end{array}\right)=\left(\begin{array}{cc}
\frac{e^{i \pi / 8}}{\sqrt{2}} & \frac{1}{2 \sqrt{2}} e^{-3 \pi i / 8} \\
\sqrt{2} e^{-3 \pi i / 8} & e^{i \pi / 8} / \sqrt{2}
\end{array}\right)
$$

The eigenvalves of this matrix are easy to compute if we realize that the $F$ move in Fig. 4 diagonalizes $B$. Hence the eigenvalves are $\bar{e}^{i 2 \pi i h_{\sigma}} e^{i \pi h_{3}}=$ $e^{-2 \pi i h_{\sigma}}$ and $e^{-2 \pi i h_{\sigma}} e^{i \pi h_{c}}$ or $e^{-i \pi / 8}$ and $e^{i \pi 3 / 8}$.

Finally to compute the physical correlation functions and the coeficients of the OPE we need to combine the left and right blocks onto monodromy invariant combinations. For these blocks where there is only one internal state this is quite straightforward. Choosing standard conventions for the two-point functions:

$$
\begin{aligned}
& \langle\varepsilon(z, \bar{z}) \varepsilon(w, \bar{w})\rangle=\frac{1}{|z-w|^{2}} \\
& \langle\sigma(z, \bar{z}) \sigma(w, \bar{w})\rangle=\frac{1}{|z-w|^{1 / 4}}
\end{aligned}
$$

we learn that $C_{\varepsilon \varepsilon}=1$ and $C_{\sigma \sigma}^{0}=1$, to determine the only nontrivial OPE coefficient $C_{\epsilon \varepsilon}^{\sigma}$, we write:

$$
\langle\sigma \sigma \sigma \sigma\rangle=A_{0}\left|\mathcal{F}_{0}^{\sigma \sigma \sigma \sigma}\right|^{2}+A_{\varepsilon}\left|\mathcal{F}_{c}^{\sigma \sigma \sigma \sigma}\right|^{2}
$$


from (2.52) and (2.56), the explicit form of the braiding matrix (3.79) and the normalization $(3.80)$ we easily find

$$
C_{\sigma \sigma}^{\varepsilon}=1 / 2
$$

From the general relation between $F$ and $B$ matrices (Fig. 9) it is easy to obtain the $F$ matrix in terms of the results (3.76) and (3.79), and it is left as an exercise.

A useful exercise in this formalism which extends to other algebras is to determine the fusion rules (3.41) using only the properties of the contour integrals defining the conformal blocks. For generic 4-point functions we will have $N$ contours of $J_{+}$and $M$ contours of $J_{-}$. Determining when some of these contour integrals contribute is equivalent to determining what internal states can appear in a block and this computes the fusion rules.

Although we have only presented the Coulomb gas representation for the Virasoro algebra, there are similar constructions for more complicated algebras. For instance, this can be done for $N=1^{44}$ and $N=2^{45}$ superconformal algebras, $W$-algebras ${ }^{46}, \mathrm{SU}(2)$ Wess-Zumino-Witten theories ${ }^{47}$ etc.

To summarize, in this section we have presented some of the general properties of degenerate conformal families, we compute the Virasoro characters and introduced a powerful computational technique based on a Coulomb gas representation with background charge related to the central term $c$ of the Virasoro algebra.

\section{Current Algebras and Wess-Zumino-Witten Models}

\subsection{Generalities}

In previous sections we have considered conformal theories whose symmetry algebra was $\operatorname{Vir} \otimes \overline{V i r}$. In general, for any CFT we will have a symmetry algebra $\mathscr{U}$, the chiral algebra $\mathscr{Q}=\mathscr{U}_{L} \times \mathfrak{U}_{R}$ with left- and righthanded componenets. In two dimensions we can label tensors as $(p, \tilde{p})$ differentials meaning that the quantity $t(z, \bar{z}) d z^{p} d \bar{z}^{p}$ is invariant under holomorphic coordinate transformations. The chiral algebras are generated by tensors of the form $(p, 0)$ and $(0, \tilde{p})$, i.e. they are either holomorphic or antiholomorphic. For a $(p, 0)$ tensor or $p$-differential the conservation law can be written as:

$$
\bar{\partial} S(z)=0 .
$$

This immediately implies an infinite number of conserved quantities,

$$
\bar{\partial}\left(z^{n} S(z)\right)=0
$$

expanding $S(z)$ in a Laurent series, the conseved quantities are the coeffcients of this expansion:

$$
S(z)=\sum_{n \in \mathbb{Z}} S_{n} z^{-n-p}
$$

In the case of Virasoro $p=2$, and $S(z)=T(z)$. The OPE of $S(z)$ with itself defines the commutation relations of the chiral algebra. Other examples are provided by the $N=1$ super-Virasoro algebra where together with $T(z)$ there is a $(3 / 2,0)$ field and $(S(z), T(z))$ are the components of a twodimensional chiral superfield. One of the characteristic features of chiral algebras is that $\mathscr{U}_{L}\left(\mathfrak{U}_{R}\right)$ always contains the identity operator and the Virasoro (Vir) algebra. The purpose of this section is to study in some detail the case when the chiral algebra is generated by $(1,0)$ fields. The simplest example is given by a free scalar field. The U(1) current is $\partial \phi$, and the OPE:

$$
\begin{aligned}
& \partial \phi(z) \partial \phi(w)=-\frac{1}{(z-w)^{2}}+\text { regular } \\
& \partial \phi(z) \quad=\sum_{n \in \boldsymbol{Z}} \alpha_{n} z^{-n-1}
\end{aligned}
$$

and the commutation relations are the canonical commutation relations for bosonic oscillators:

$$
\left[\alpha_{n}, \alpha_{m}\right]=n \delta_{n+m, 0}
$$

Another example is provided by a set of Weyl-Majorana fermions $\psi_{i}(z)$. Given a group $G$ and a real representation $\left(T^{a}\right)_{i j}, i, j=1,2, \ldots \operatorname{dim} T$ we can construct currents:

$$
j^{a}(z)=\frac{1}{2}: \psi T^{a} \psi:=\sum_{n \in \mathbb{Z}} j_{n}^{a} z^{-n-1} .
$$

Since the only nontrivial $\mathrm{OPE}$ is:

$$
\psi_{i}(z) \psi_{j}(w)=\frac{\delta_{i j}}{z-w}+\text { regular }
$$


the commutation relations of the $J_{n}^{a}$ 's follow from the OPE:

$$
j^{a}(z) j^{b}(w)=\frac{\operatorname{tr} T^{a} T^{b} / 2}{(z-w)^{2}}+i f^{a b c} \frac{j^{c}(w)}{z-w}+\text { reg. }
$$

where $f^{a b c}$ are the structure constants of the group $G$ :

$$
\left[T^{a}, T^{b}\right]=i f^{a b c} T^{c} .
$$

In terms of commutators (4.8) becomes:

$$
\left[j_{n}^{a}, j_{m}^{b}\right]=i f^{a b c} j^{c} n+m+\frac{1}{2} \operatorname{tr} T^{a} T^{b} n \delta_{n+m, 0}
$$

It is also useful to compute the OPE between the current and $\psi_{i}(z)$ :

$$
j^{a}(z) \phi_{k}(w)=-\left(T^{a}\right)_{k j} \frac{\psi_{j}(w)}{z-w} .
$$

From (4.10) we see that the zero modes $j_{0}^{a}$ satisfy the commutation relation of the Lie algebra of $G$. If we consider complex fermions, the action for the left movers is:

$$
S=\frac{1}{\pi} \int b^{i} \tilde{\partial} c_{i} \quad i=1, \ldots, N
$$

with two-point functions:

$$
\begin{aligned}
& b^{i}(z) c_{j}(w)=\frac{\delta_{j}^{i}}{z-w}+\text { regular } \\
& c_{i}(z) b^{j}(w)=\frac{\delta_{i}^{j}}{z-w}+\text { regular } .
\end{aligned}
$$

Given representation $T^{\alpha}$ of $G$ of dimension $N$ we can write

$$
j^{a}(z)=b^{i}\left(T^{\alpha}\right) i^{j} c_{j}=b T^{a} c
$$

and

$$
j^{a}(z) j^{b}(w)=\frac{\operatorname{tr} T^{a} T^{b}}{(z-w)^{2}}+\frac{1}{z-w} i f^{a b c} J^{c}(w)+\text { regular }
$$

Normalizing the roots of $G$ so that the highest root $\theta$ is such that $\theta^{2}=2$, and choosing an appropriate basis for the Lie algebra, tr $T^{a} T^{b}=k \delta^{a b}$.
Choosing this basis we can always write down the commutation relations of the current algebra as:

$$
\left[j_{n}^{a}, j_{m}^{b}\right]=i f^{a b c} j_{n+m}^{c}+n \delta^{a b} k \delta_{n+m, 0}
$$

In the same way that the Virasoro generators provide a projective representation of the algebra of vector fields $l_{n}$, the $j_{n}^{a}$ are associated to the loop algebra of $G$ (for more details and references on the theory of Kac-Moody algebras and their applications, see Ref. 48):

$$
\varepsilon(z)=\sum_{a, n} \varepsilon_{n}^{a} T^{a} z^{n}
$$

$\varepsilon_{n}^{a}$ are the generators of the loop algebra. The algebra (4.16) is a central extension of the loop algebra of $G$. This is the case when the conserved currents generating the chiral algebra have dimensions $(1,0)$ or $(0,1)$. Their commutation relations always generate a Kac-Moody algebra like (4.16). If the ground state is invariant under the Kac-Moody algebra:

$$
j_{n}^{\alpha}|0\rangle=0 n \geq 0
$$

the fields of the theory will organize into families each providing an irreducible representation of the Kac-Moody algebra. As with Virasoro we will have primary and descendant fields. A primary field has the simplest transformation properties:

$$
j^{a}(z) \phi_{j}(w)=-\left(T^{a}\right)_{j k} \frac{\phi_{k}(w)}{z-w}
$$

or:

$$
\left[j_{n}^{a}, \phi_{j}(w)\right]=-\left(T^{a}\right)_{j k} \phi_{k}(w) w^{n}
$$

The module generated by $\phi_{j}(w)$ has a highest weight subspace

$$
|j\rangle=\phi_{j}(0)|0\rangle
$$

forming a representation of the group $G$, and the descendants are obtained acting on $|j\rangle$ with $j_{-n}^{a} n>0$.

$$
V_{\left(\phi_{j}\right)}=\left\{j_{-n_{1}}^{a_{1}} \ldots j_{-n_{N}}^{a_{n}}|j\rangle n_{1}, \ldots, n_{N}>0, N \geq 0\right\}
$$


The primary fields also have the OPE:

$$
\begin{aligned}
& T(z) \phi_{i}(w)=\frac{h}{(z-w)^{2}} \phi_{i}(w)+\frac{1}{z-w} \partial_{w} \phi_{i}(z)+\ldots \\
& T(z) j^{a}(w)=\frac{1}{(z-w)^{2}} j^{a}(w)+\frac{1}{(z-w)} \partial^{a}(w)+\text { regular } .
\end{aligned}
$$

From the basic OPE between currents:

$$
j^{a}(z) j^{b}(w)=\frac{k \delta^{a b}}{(z-w)^{2}}+i f^{a b c} \frac{j^{c}(w)}{z-w}+\text { reg. } .
$$

we can use $\mathrm{SL}_{2}$ invariance and Bose symmetry to compute the 2,3 and 4-point functions involving only currents:

$$
\begin{aligned}
& \left\langle J^{a}(z) j^{-b}(w)\right\rangle=\frac{k \delta^{a b}}{(z-w)^{2}} \\
& \left\langle j^{a}(z) j^{b}(w) j^{c}(y)\right\rangle=\frac{i k f^{a b c}}{(z-w)(z-y)(w-y)} \\
& \left\langle j^{a}(z) j^{b}(w) J^{c}(x) j^{d}(y)\right\rangle \\
& =k^{2}\left(\frac{\delta^{a b} \delta^{c d}}{(z-w)^{2}(x-y)^{2}}+\frac{\delta^{a c} \delta^{b d}}{(z-x)^{2}(w-y)^{2}}+\frac{\delta^{a d} \delta^{b c}}{(z-y)^{2}(w-x)^{2}}\right) \\
& -\frac{k}{3} \frac{f^{a b e} f^{c d e}+f^{a c e} f^{b d e}}{(z-w)(z-x)(w-y)(x-y)}-\frac{k}{3} \frac{f^{a b e} f^{c d e}+f^{a d e} f^{c b e}}{(z-w)(z-y)(w-x)(x-y)} \\
& -\frac{k}{3} \frac{f^{a c e} f^{b d e}+f^{a d e} f^{b c e}}{(z-x)(z-y)(x-w)(w-y)} \cdot
\end{aligned}
$$

The structure of (4.24c) is fixed by the pole structure forced by the $\mathrm{OPE}$ and requiring symmetry under arbitrary exchanges of $(a, z),(b, w)$, $(c, x),(d, y)$.

Given a Kac-Moody algebra one can always obtain a Virasoro algebra. The energy-momentum tensor is constructed using Sugawara's prescription. From the U(1) case $j(z)=\partial \phi(z)$, (see Ref. 48 for more details), we know that $T(z)=-\frac{1}{2}: j(z) j(z):$. It is reasonable to try and generalize this prescription in the nonabelian case. Define the normal ordering between two currents by:

$$
: j^{2}(z):=\lim _{z \rightarrow w}\left(\sum_{a} j^{a}(z) j^{a}(w)-\frac{k \operatorname{dim} G}{(z-w)^{2}}\right)
$$

Using (4.25) we learn immediately that for:

$$
T(z) j(w)=\frac{j(w)}{(z-w)^{2}}+\frac{1}{z-w} \partial j(w)+\ldots
$$

to hold, we need to normalize $(4.26)$ as:

$$
T(z)=\frac{1}{2 k+c_{G}}: j^{2}(z):
$$

where $c_{G}$ is the quadratic casimir in the adjoint representation. From (4.24c) we can compute the central extension of the Virasoro algebra generated by $T(z)$. If we define the level as $x=2 k / \theta^{2}$, then

$$
\begin{aligned}
& c=\frac{x \operatorname{dim} G}{x+\tilde{h}(G)} \\
& \tilde{h}(G)=c_{G} / \theta^{2}
\end{aligned}
$$

$\tilde{h}$ is the dual Coxetor number of $G$ and it is independent of the way we normalize the roots. Hence in any theory with Kac-Moody symmetry we can have three types of null vectors: a) Purely Kac-Moody. b) Purely Virasoro, c) mixed. The constraints imposed by null-vectors of type a) are derived from the Ward identity ${ }^{12}$

$$
\left\langle j^{a}(z) \phi_{i_{1}}\left(z_{1}\right) \ldots \phi_{i_{N}}\left(z_{N}\right)\right\rangle=-\sum_{j=1}^{N} \frac{\left(T^{a}\right)}{z-z_{j}} i_{j} k_{j}\left\langle\phi_{i_{1}} \ldots \phi_{k_{j}} \ldots \phi_{i_{N}}\right\rangle
$$

The mixed ones are very important. Their decoupling leads to the KnizhnikZamalodchikov equation for the conformal blocks of the theory. Expanding (4.26) in powers of $z, L_{-1}$ is given by:

$$
L_{-1}=\frac{1}{2 k+c_{G}} \sum_{a} \sum_{n}: j_{n-1}^{a} j_{-n}^{a}: \text {. }
$$

For any primary state then $L_{-1}$ minus the right-hand side of (4.30) always gives a mixed Virasoro Kac-Moody null vector. As any null vector, it should be annihilated by $L_{1}$ and $j_{1}^{a}$ :

$$
\begin{aligned}
& {\left[L_{1},\left(L_{-1}-\frac{1}{2 k+c_{G}} j_{-1}^{b} j_{0}^{b}\right)\right]|i\rangle=0} \\
& {\left[j_{1}^{a},\left(L_{-1}-\frac{1}{2 k+c_{G}} j_{-1}^{b} j_{0}^{b}\right)\right]|i\rangle=0}
\end{aligned}
$$


and we have used the highest weight state condition for $|i\rangle$. The second Casimir in a given representation $T^{a}$ is defined by:

$$
\sum_{a}\left(T^{\alpha} T^{\alpha}\right) i j=c_{2}(T) \delta_{i j}
$$

Then from:

$$
\left[L_{n}, j_{m}^{a}\right]=-m j_{n+m}^{a}
$$

(a consequence of the OPE $T(z) j^{a}(w)$ ) we easily obtain from (4.30) the dimension of the primary state $|i\rangle^{12}$ :

$$
h=\frac{c_{2}(T)}{2 k+c_{2}(G)} .
$$

In particular, for $\mathrm{SU}(2) \theta=\sqrt{2}$, and the highest weights are of the form $j \theta, j=0,1 / 2,1, \ldots ;$ then $c_{2}(j \theta)=2 j(j+1)$ and:

$$
h(j)=\frac{j(j+1)}{k+2}
$$

The general formula for a simple group $G$ is easy to derive. Normalizing the long roots to have length 2 , the quadratic Casimir as an operator is:

$$
Q=\sum_{i} H_{i}^{2}+\sum_{\alpha} \frac{\alpha^{2}}{2} E_{\alpha} E_{-\alpha}
$$

where $H_{i}$ are the generators of the Cartan subalgebra and $\alpha$ runs over the roots of the algebra. For simplizity take $G$ to be simple laced. Then for a representation with highest weight $|\lambda\rangle$ we have:

$$
\begin{aligned}
& Q|\lambda\rangle=(\lambda, \lambda+2 \rho)|\lambda\rangle \\
& \rho \quad=\frac{1}{2} \sum_{\alpha>0} \alpha .
\end{aligned}
$$

Hence in general if $\phi_{\lambda}(z)$ is a primary field labelled by a highest weight

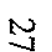
representation $\lambda$ its conformal dimension is:

$$
h=\frac{(\lambda, \lambda+2 \rho)}{k+\tilde{h}(g)}
$$

Next we use (4.30) to derive a differential equation satisfied by the correlation functions of primary fields. Let $\phi_{1}\left(z_{1}\right) \ldots \phi_{n}\left(z_{n}\right)$ be primary fields,

$$
\begin{array}{ll}
|i\rangle=\phi_{i}(0)|0\rangle & L_{0}|i\rangle=\frac{c(i)}{k+\tilde{h}}|i\rangle \\
j_{0}^{a}|i\rangle=-\left(T^{\alpha}\right)_{i j}|j\rangle & L_{n}|i\rangle=j_{n}^{\alpha}|i\rangle=0 \quad n>0 .
\end{array}
$$

Since

$$
\left(L_{-1}-\frac{1}{2 k+c_{G}} J_{-1}^{a} J_{0}^{a}\right) \phi(z)=0
$$

and:

$$
\left(J_{-1}^{a} \phi\right)(z)=\oint_{z} d w \frac{1}{w-z} J^{a}(w) \phi(z)
$$

we obtain:

$$
\begin{aligned}
\left\langle\phi_{1}\left(z_{1}\right) \ldots\left(L_{-1}-\frac{1}{2 k+c_{G}} J_{1}^{a} J_{0}^{a}\right) \phi_{i}\left(z_{i}\right) \ldots \phi_{N}\left(z_{N}\right)\right\rangle & =0 \\
\left(\left(2 k+c_{G}\right) \frac{\partial}{\partial z_{i}}+\sum_{j \neq i} \frac{T_{i}^{a} T_{j}^{a}}{z_{i}-z_{j}}\right)\left\langle\phi_{i}\left(z_{1}\right) \ldots \phi_{N}\left(z_{N}\right)\right\rangle & =0
\end{aligned}
$$

This is the Knizhnik-Zamalodchikov ${ }^{12}$ equation for the conformal blocks.

A field theory which contains all the features we have explained so far, is the Wess-Zumino-Witten (WZW) $\sigma$-model ${ }^{11}$. Its action is

$$
S=\frac{k}{16 \pi} \int_{S^{2}} \operatorname{tr} \partial_{\mu} g^{-1} \partial^{\mu} g d^{2} \sigma+\frac{k}{24 \phi} \int_{D, \partial D=S^{2}} \operatorname{tr}\left(g^{-1} d g\right)^{3} .
$$

$g$ is a field valued in the group $G, D$ is a three-dimensional disc whose boundary is the two dimension space-time and $k$ is an integer. The action is invariant under the infinite dimension Kac-Moody group $\hat{G}_{L} \times \hat{G}_{k}$ :

$$
g\left(x^{+}, x^{-}\right) \rightarrow \Omega\left(x^{+}\right) g\left(x^{+}, x^{-}\right) \bar{\Omega}^{-1}\left(x^{-}\right)
$$

with infinitesimal generators given by

$$
\begin{aligned}
& j^{a}(z) T^{a}=-\frac{i k}{2} \partial_{z} g g^{-1} \\
& j^{\alpha}(\bar{z}) T^{a}=-\frac{i k}{2} g^{-1} \partial_{\bar{z}} g
\end{aligned}
$$


$\infty \quad$ the central extension is $k$, and the energy-momentum tensor is a current bilinear given by the Sugawara construction (4.26).

\subsection{Representations and fusion rules}

We can rewrite the defining relations (4.15) in terms of a Cartan-Weyl basis. If $G$ is a simple algebra, $H^{i}$ is a basis for the Cartan subalgebra $i=1, \ldots, r=\operatorname{rank} G$, and $\alpha$ labels the roots of $G$, we can write (for many more details on Kac-Moody algebras see Ref. 49):

$$
\begin{aligned}
{\left[H_{n}^{i}, H_{m}^{i}\right] } & =k \delta^{i j} n \delta_{n+m, 0} \\
{\left[H_{n}^{i}, E_{m}^{\alpha}\right] } & =\alpha^{i} E_{n+m}^{\alpha} \\
{\left[E_{m}^{\alpha}, E_{n}^{\beta}\right] } & =N(\alpha, \beta) E_{m+n}^{\alpha+\beta} \quad \alpha+\beta \text { a root } \\
& =0 \quad \alpha+\beta \text { not a root } \\
& =2 \frac{\alpha^{i} H_{n+m}^{i}}{\alpha^{2}}+\frac{2 k m}{\alpha^{2}} \delta_{n+m, 0} \quad \beta=-\alpha \\
{\left[k, j_{n}^{\alpha}\right] } & =0, \quad d=-L_{0},\left[d, j_{n}^{\alpha}\right]=n j_{n}^{a},[d, k]=0 .
\end{aligned}
$$

We have included the "level" operator $d$ to avoid having an infinite degeneracy for the roots. The maximal set of commutating operators in (4.42) are $\left(H_{0,}^{i}, k, d\right)$, and the roots of the Kac-Moody algebra $\hat{G}$ can be read off from (4.42) to be $(\alpha, 0, n) n \in \mathbb{Z}, \alpha$ a root of $G$. There are $r+1$ simple roots. A root $(\alpha, 0, n)$ is positive if $n>0$ or if $\alpha>0$ for $n=0$. If $\alpha_{i} i=1, \ldots, r$ are the simple roots of $G$, the simple roots of $\hat{G}$ are $\left(\alpha_{i}, 0,0\right) \equiv a_{i}$ and $(-\theta, 0,1)=a_{0},(\theta$ is as before the highest root). The scalar product on the space of weights is:

$$
\left(\boldsymbol{\lambda}_{1}, a_{1}, b_{1}\right) \cdot\left(\boldsymbol{\lambda}_{2}, a_{2}, b_{2}\right)=\lambda_{1} \cdot \lambda_{2}+a_{1} b_{2}+a_{2} b_{1}
$$

and the Dynkin diagram follows from

$$
K_{i j}=2 a_{i} \cdot a_{j} / a_{j}^{2} \quad i, j=0,1, \ldots, r
$$

as for ordinary lie algebras, we draw a dot on the diagram for each simple root and if $K_{i j} \neq 0$ we draw $K_{i j} K_{j i}$ lives joining dots $i$ and $j$. One needs the relation

$$
\theta / \theta^{2}=\sum_{i=1}^{5} m_{i} \alpha_{i} / \alpha_{i}^{2}
$$
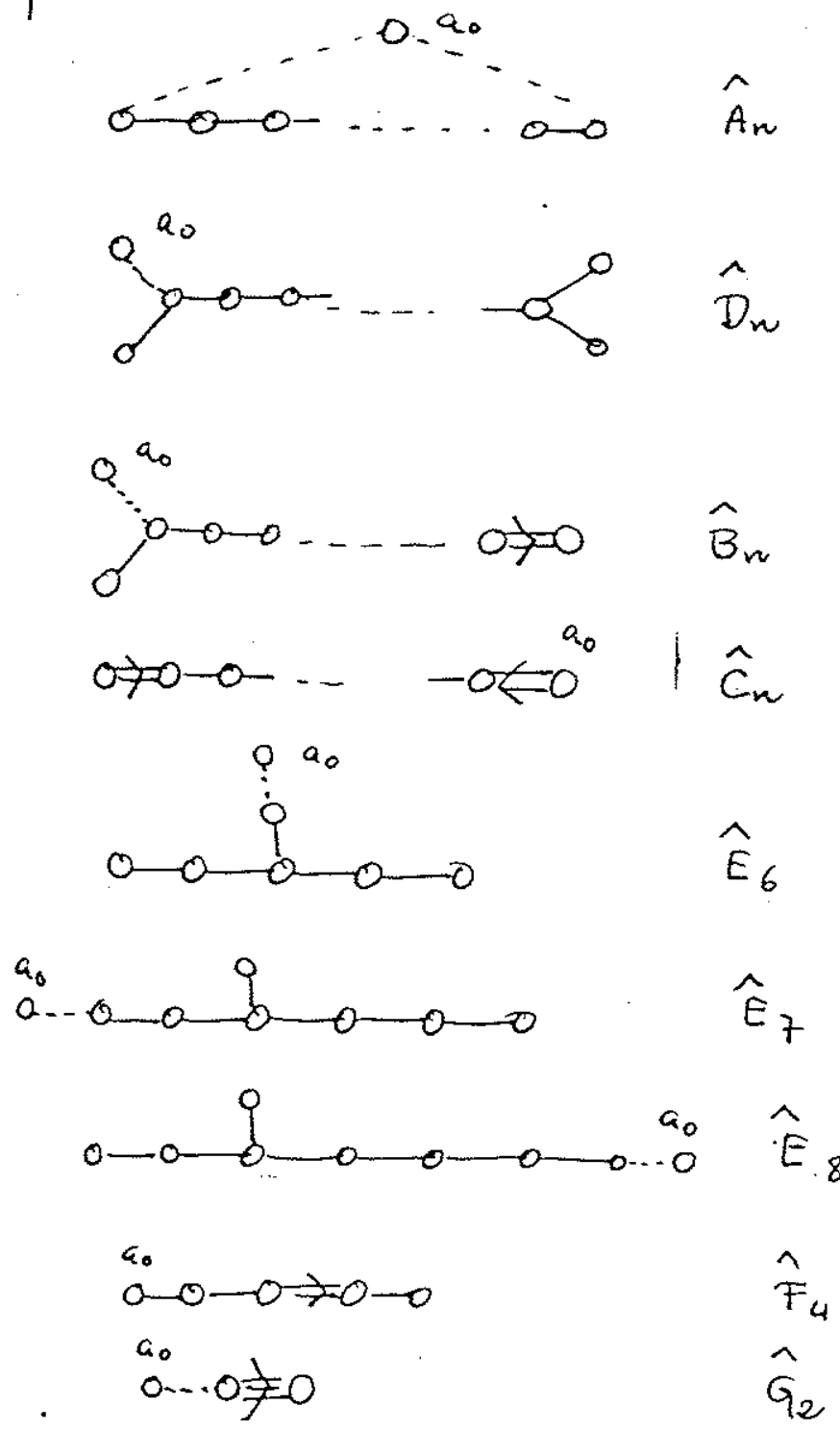

Fig. 14. Dynkin diagrams for untwisted affine Kac-Moody algebras.

to obtain the Dynkin diagrams in Fig. 14.

The highest weight representations are constructed as tensor product of $r+1$ - fundamental representations. The weights are defined as in the 
classical case:

$$
2 \Lambda_{i} \cdot a_{j} / a_{j}^{2}=\delta_{i j} \quad i, j=0,1, \ldots, r .
$$

It is not difficult to show that

$$
\begin{aligned}
& \Lambda_{i}=\left(\lambda_{i}, m_{i} \frac{\theta^{2}}{2}, 0\right) \quad i=1, \ldots, r \\
& \Lambda_{0}=\left(0, \frac{1}{2} \theta^{2}, 0\right)
\end{aligned}
$$

( $m_{i}$ is defined in (4.44). Any highest weight vector representation of $\hat{G}$ is a positive integer combination of $\Lambda_{0}, \ldots, \Lambda_{r}$ :

$$
\mu=n_{0} \Lambda_{0}+\sum_{i=1}^{r} n_{i} \Lambda_{i} \quad n_{0}, n_{i} \geq 0
$$

for unitary representations, $k \geq 0$. This is proved by computing:

$$
\left\|E_{-1}^{\alpha} \mid \mu>\right\|^{2}=\left\langle\mu\left|E_{1}^{-\alpha} E_{-1}^{\alpha}\right| \mu\right\rangle=\frac{2}{\alpha^{2}}(k-\alpha \cdot \mu) \geq 0
$$

Since this holds for any root $\alpha$ we conclude that $k \geq 0$. The quantity $2 k / \theta^{2} \equiv x$ is the level of the Kac-Moody algebra. Important representations of $G$ are those with level one. Normalizing $\theta^{2}=2$, for convenience, we see from (4.46) that $\Lambda_{0}$ (the basic representation) is always of level one. The level one representations depend on the algebra. For example, in $\hat{A}_{n}$ all the basic representations are level one. In $\hat{D}_{n}$ we have the basic as well as the two spinors and the fundamental representation all with level 1. For $E_{8}$ only the basic representation has level one. This is of great importance in the construction of the heterotic string ${ }^{50}$. To summarize, every highest weight representation of $\hat{G}$ is labelled by a highest weight of the classical algebra and an integer (the level). $\Lambda=(\lambda, k, 0)$.

We come next to determing which representations will appear in the WZW models (or in any unitary CFT whose chiral algebra is $\hat{G}_{L} \times \hat{G}_{k}$ ) and the fusion rules of the operator algebra. We follow closely the presentation in Ref. 13.

To the simple roots of $\hat{G}$ we can associate a Chevalley basis for $\hat{G}$. This simply means that we can construct some SU(2) subalgebras of $\hat{G}$. If N $r=\operatorname{rank} G, r$ of these subalgebras are ordinary $\mathrm{SU}(2)$ subalgebras of $G$. The more interesting $\mathrm{SU}(2)$ is the one associated to $a_{0} ; E_{1}^{-\theta}$. Then:

$$
\left[E_{-1}^{\theta}, E_{1}^{-\theta}\right]=\theta \cdot H_{0}-k \text {. }
$$

Defining:

$$
x^{+} \equiv E_{1}^{-\theta} \quad x^{-}=E_{-1}^{\theta} \quad x^{3}=k-\theta \cdot H_{0}
$$

we obtain:

$$
\begin{aligned}
& {\left[x^{3}, x^{ \pm}\right]= \pm 2 x^{ \pm}} \\
& {\left[x^{+} . x^{-}\right]=x^{3} .}
\end{aligned}
$$

If $\phi_{\lambda}$ is the highest weight component of a primary field, then $x^{+} \phi_{\lambda}=0$ and we can construct the $\mathrm{SU}(2)$ representation:

$$
\begin{aligned}
& \phi_{\lambda}, x^{-} \phi_{\lambda}, \ldots \\
& x^{3} \phi_{\lambda}=(k-\langle\lambda, \theta\rangle) \phi_{\lambda} ;\langle\lambda, \theta\rangle \equiv 2 \lambda \cdot \theta / \theta^{2} .
\end{aligned}
$$

The representation is finite and integrable if $x^{3}$ has integer eigenvalues larger or equal to zero. Hence:

$$
k \geq\langle\lambda, \theta\rangle
$$

and the dimension of this $\mathrm{SU}(2)$ representation is $k-\langle\lambda, \theta\rangle+1$. Considering instead the operators $E_{0}^{\theta}, E_{0}^{-\theta} H_{0}^{\theta}$, they also generate an $\mathrm{SU}(2)$ subalgebra, then $\left(J_{0}^{-\theta}\right)^{\langle\lambda, \theta\rangle+1} \phi_{\lambda}=0$. Since $k \geq\langle\lambda, \theta\rangle$ and $\left.\langle\lambda, \theta\rangle \geq\langle\lambda, \alpha\rangle, \alpha\right\rangle 0$, we obtain $\left(J_{0}^{-\alpha}\right)^{(\lambda, \alpha)+1} \phi_{\lambda}=0$; and the irreducible representation is integrable iff:

$$
\left(J_{0}^{-8}\right)^{k+1} \phi_{\lambda}=0
$$

Furthermore, we have a null vector in this integrable representation:

$$
\left(X^{-}\right)^{k-\langle\lambda, \theta\rangle+1} \phi_{\lambda}=0 \text {. }
$$

This null vector allows us to derive the fusion rules of the model. Since

$$
\left(J_{+n}^{a} \phi\right)(z)=\oint_{z} d w(w-z)^{n} j^{a}(w) \phi(z)
$$

we can write

$$
\begin{aligned}
0= & \left\langle\left(x^{-}\right)^{k-\langle\lambda, \theta)+1} \phi_{\lambda}(z) \phi_{1}\left(z_{1}\right) \ldots \phi_{n}\left(z_{n}\right)\right\rangle \\
= & \sum_{\substack{l_{1} \ldots, l_{n} \\
l_{1}+\ldots+l_{n}=k-\langle\lambda, \theta\rangle+1}} \frac{(k-\langle\lambda, \theta\rangle+1) !}{l_{n} ! l_{2} ! \ldots \ldots l_{n} !} \\
& \frac{\left(T_{1}^{\theta}\right)^{l_{1}} \ldots\left(T_{n}^{\theta}\right)^{l_{n}}}{\left(z-z_{1}\right)^{l_{1}}\left(z-z_{2}\right)^{l_{2}} \ldots\left(z-z_{n}\right)^{l_{n}}}\left\langle\phi_{\lambda}(z) \phi_{1}\left(z_{1}\right) \ldots \phi_{n}\left(z_{n}\right)\right\rangle=0 .
\end{aligned}
$$


A consequence of this Ward identity is the decoupling of nonintegrable representations from the correlating functions. If $\lambda=i d$, the correlation function in (4.55) is independent of $z$. If $\phi_{r}\left(z_{r}\right)$ is a nonintegrable primary field, in (4.55) with $\lambda=i d$ multiplied by $\left(z-z_{r}\right)^{k}$ and integrable along a small contour containing $z_{r}$ and no other $z_{i}$ 's. Then:

$$
\left(T_{r}^{\theta}\right)^{k+1}\left\langle\phi_{\lambda}(z) \ldots \phi_{r}\left(z_{r}\right) \ldots\right\rangle=0 .
$$

If $\phi_{\lambda}$ is integrable, $\left(J_{0}^{-\theta}\right)^{k+1} \phi_{\lambda}=0$. For nonintegrable ones, if $\phi^{\lambda_{r}}\left(z_{r}\right)$ is the highest weight state, then there is some state $\tilde{\phi}_{r}\left(z_{r}\right)=\left(J_{0}^{-\theta}\right)^{k+1} \phi^{\lambda_{r}}\left(z_{r}\right)$ $\neq 0$, taking $\phi_{r}\left(z_{r}\right)=\tilde{\phi}_{r}\left(z_{r}\right)$ in (4.56) we find that the correlation functions involving $\phi_{r}\left(z_{r}\right)$ vanish for $\phi_{r}$ a nonintegrable representation. We next use (4.55) for the three-point function:

$$
\sum_{\substack{l_{1}, l_{2} \\ l_{1}+l_{2}=k-\langle\lambda, \theta\rangle+1}} \frac{(M+1) !}{l_{1} ! l_{2} !} \frac{\left(T_{1}^{\theta}\right)^{l_{1}}\left(T_{2}^{\theta}\right)^{l_{2}}}{\left(z-z_{1}\right)^{l_{1}}\left(z-z_{2}\right)^{l_{2}}}\left\langle\phi_{\lambda}(z) \phi_{1}\left(z_{1}\right) \phi_{2}\left(z_{2}\right)\right\rangle=0
$$

Since $\mathrm{SL}_{2}$ invariance determines the $z$-dependence of the three-point function we obtain:

$$
\left(T_{1}^{\theta}\right)^{l_{2}}\left(T_{2}^{\theta}\right)^{l_{2}}\left\langle\phi_{\lambda}(z) \phi_{1}\left(z_{1}\right) \phi_{2}\left(z_{2}\right)\right\rangle=0, l_{1}+l_{2} \geq k-\langle\lambda, \theta\rangle+1 .
$$

In a highest weight representation $V(\Lambda)$ of $G$ with highest weight $\Lambda, \lambda \in$ $V(A)$ the depth of $\lambda$ is defined as the largest integer $j$ such that $\lambda-j^{\theta} \in$ $V(\Lambda)$. Then the three-point function $\left\langle\phi_{A}(z) \phi_{1}^{i}\left(z_{1}\right) \phi_{2}^{j}\left(z_{2}\right)\right\rangle$ vanishes identically unless either $f_{A_{i j}}=0$ ( $f$ is the Clebsch-Gordan coefficient) or

$$
\operatorname{depth}(i)+\operatorname{depth}(j) \leq k-\langle\lambda, \theta\rangle
$$

Let $f_{\lambda_{i}} \neq 0$. If $n=\operatorname{depth}(i), m=\operatorname{depth}(j)$ and $\left\langle\phi_{\Delta} \phi_{i} \phi_{j}\right\rangle \neq 0$ for $n+m \geq$ $k-\langle\lambda, \theta\rangle+1$ then we obtain the contradiction:

$$
\begin{aligned}
0 & \neq\left\langle\phi_{\Lambda}(z) \phi_{1}^{i}\left(z_{1}\right) \phi_{2}^{i}\left(z_{2}\right)\right\rangle \\
& =c\left(T_{1}^{\theta}\right)^{n}\left(T_{2}^{\theta}\right)^{m}\left\langle\phi_{\Lambda} \phi_{1}^{(i-n \theta)} \phi_{2}^{(j-m \theta)}\right\rangle=0
\end{aligned}
$$

from (4.58). Hence (4.59) provides the fusion rules for arbitrary WZW. models. As an example we consider first $\mathrm{SU}(2)_{k}$. Then $\theta=\sqrt{2}, \lambda=j^{\theta}, j \in$ $\mathbb{Z} / 2$ and the integrable representations satisfy $k \geq\langle\lambda, \theta\rangle=z_{j}$ i.e. $j=$
$0,1 / 2,1, \ldots, k / 2$ and these are $k+1$ irreducible integrable representation. If $m$ is the third component of spin in the spin $j$ representation, then depth $(m)=m+j$. This and (4.59) imply:

$$
\left.\begin{array}{l}
\mu_{1}=m_{1} \theta \\
\mu_{2}=m_{2} \theta
\end{array}\right\} \quad \operatorname{depth}\left(\mu_{1}\right)+\operatorname{depth}\left(\mu_{2}\right)=m_{1}+m_{2}+j_{1}+j_{2} \leq k-2 j_{3} .
$$

Since $m_{1}+m_{2}+m_{3}=0$ (we are taking $\Lambda$ in (4.58) to be $\left.j_{3} \theta\right),(j-3-$ $\left.m_{3}\right)+\left(j_{1}+j_{2}+j_{3}\right) \leq k$ leading to

$$
j_{1}+j_{2}+j_{3} \leq k .
$$

Hence

$$
\left[\phi_{j_{1}}\right] \times\left[\phi_{j_{3}}\right]=\sum_{\left.j_{3}=\mid j_{1}-j_{2}\right]}^{\min \left(j_{1}+j_{2}, k-j_{1}-j_{2}\right)}\left[\phi_{j}\right]
$$

are the fusion rules for $\mathrm{SU}(2)$ level $k$ WZW models. With (4.11) it is easy to check that the fusion rules for the minimal models (3.43) are a pair of (4.61) rules: If in the $\phi_{(n, m)}$ field we identify $n=2 j+1, m=2 j^{r}+1$ and $p=k+2, q=k^{\prime}+2$ the $\mathrm{SU}(2) k \times \mathrm{SU}(2) k^{\prime}$ fusion rules (4.61) generate (3.43).

For other groups, say $\mathrm{SU}(N)$ the computation of the depth of a given weight is a complicated algebraic problem in general. The admissible $\mathrm{SU}(N)$ representations are characterized by those Young tableaux $\left[\lambda_{1}, \lambda_{2}\right.$ $\left., \ldots, \lambda_{N}\right], \lambda_{1} \geq \lambda_{2} \geq \ldots \geq \lambda_{N}$ such that $\lambda_{1}-\lambda_{N} \leq k$. Examples are given in Fig. 15. The fusion rules are easy to work in some cases. For instance, if one of the representations is the fundamental representation. The weights in the $N$-dimensional representation of $\mathrm{SU}(N)$ are $e_{1}, \ldots, e_{N}$ satisfying

$$
\begin{aligned}
& e_{1}+\ldots+e_{N}=0 \\
& e_{i} \cdot e_{j}=-\frac{1}{N} \quad i \neq j \quad e_{i}^{2}=\frac{N-1}{N}
\end{aligned}
$$

The nonzero roots are:

$$
\pm\left(e_{i}-e_{j}\right) \quad i<j
$$

and the basic weights are:

$$
\Lambda_{1}=e_{1} \Lambda_{2}=e_{1}+e_{2}, \ldots, \Lambda_{k}=e_{1}+\ldots+e_{k} \quad k \leq N
$$


a)
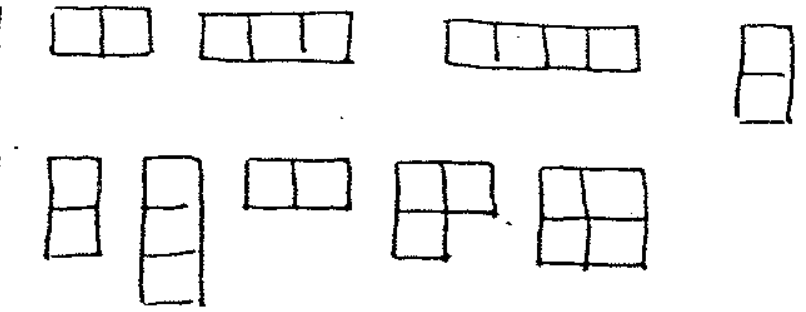

b)

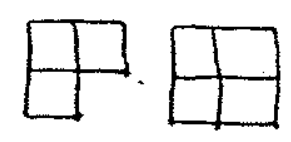

Fig. 15. a) Integrable represenatations in $\mathrm{SU}(2)$ at level 4. b) Integrable representation of $\mathrm{SU}(3)$ level 2.

Choosing the simple roots as:

$$
\alpha_{i}=e_{i}-e_{i+1} \quad 1 \leq i \leq N-1
$$

we find that

$$
\Lambda_{i} \cdot \alpha_{j}=\delta_{i j}
$$

The weight $\Lambda k$ in (4.64) is represented by a Young tableaux with a single column and $k$ boxes. A general highest weight takes the forms:

$$
\Lambda=n_{1} \Lambda_{1}+\ldots+n_{N-1} \Lambda_{N-1}=f_{1} e_{1}+f_{2} e_{2}+\ldots+f_{N-1} e_{N-1}
$$

represented by a tableaux with $f_{1}$ boxes in the first row, $f_{z}$ in the second etc, $f_{1} \geq f_{2} \geq \ldots \geq f_{N-1}$. The highest root $\theta=e_{1}-e_{N}$, then $\Lambda \cdot \theta=f_{1} \leq k$ (for level $k$ in $\mathrm{SU}(N)$ ). A tableaux is regular if $f_{N}=0$. If $f_{N} \neq 0$, then $\left[f_{1}, \ldots, f_{N}\right] \simeq\left[f_{1}-f_{N}, f_{2}-f_{N}, \ldots, f_{N-1}-f_{N, 0}\right]$ because the Young diagrams consisting of a coloumn with $N$ boxes is the trivial representation. The weights of the representation $\Lambda k$ are $e_{i_{1}}+\ldots+e_{i_{k}} 1 \leq i_{1}<i_{2}<\ldots<$ $i_{k} \leq N$ and

$$
\begin{array}{ll}
\operatorname{depth}\left(e_{1}+e_{i_{2}}+\ldots+e_{i \pi}\right)=1 \\
=\operatorname{depth}(\mu)=0 \quad \text { otherwise }
\end{array}
$$

In $\mathrm{SU}(N)$ many of its irreducible representations are complex. The tableaux

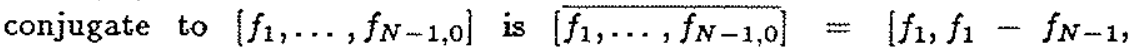
$\left.f_{1}-f_{N-2}, \ldots, f_{1}-f_{2,0}\right]$. Hence to find out if $\left[f_{i}\right]$ is the OPE $\left[f_{i}^{\prime}\right] \times[1]$ we have to look at the three-point function:

$$
\left\langle\phi_{\overline{\left[f_{1}\right]}} \phi_{\left[f_{i}^{\prime}\right]} \phi_{[1]}\right\rangle \text {. }
$$

The conclusion is the following. If $f_{1}^{\prime}+1 \leq k$ the fusion rules are identical to the decomposition rules of tensor products of tableaux. When $f_{1}^{\prime}=k$,

then the decomposition will only include tableaux with $f_{1}^{\prime}=k$ and apart from that we follow the standard rules. Since for $\mathrm{SU}(N)$ every field can be obtained by decomposing the tensor product of a sufficient number of fundamental fields, it is often useful to collect the fusion rules in Bratelli diagrams. They describe the decomposition of products between the fundamental and other representations. In Fig. 16 we present as an example $\mathrm{SU}(2)_{2}$ and in Fig. $17 \mathrm{SU}(3)_{2}$. The first row in both diagrams is the fundamental representation. The second row represents the result of the $O P E$ of the first row with the fundamental representation; the third row is the result of computing the OPE of the second row with the fundamental etc. Since for finite $k$ the number of integrable representations of $\hat{G}_{k}$ is finite, the Bratelli diagram will repeat after a finite number of steps. For $\mathrm{SU}(2)_{k}$ these diagrams can be summarized also in terms of Dynkin-like diagrams. The primary fields have $j=0,1 / 2,1, \ldots, k / 2$. Each one can be associated

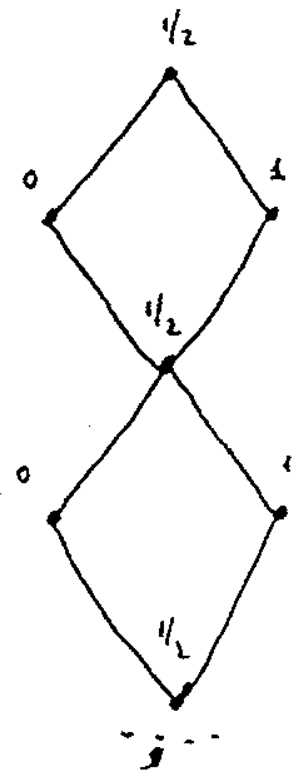

Fig. 16. Bratteli diagram for $\mathrm{SU}(2)_{2}$.

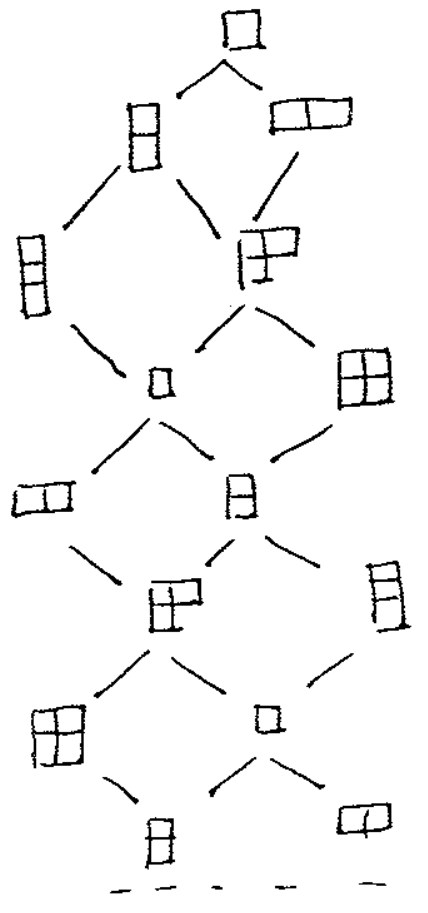

Fig. 17. Bratteli diagram for $\mathrm{SU}(3)_{2}$. 


$$
\begin{array}{llllllll}
0 & 1 / 2 & 1 & j-1 / 2 & j & j+2 & \frac{k}{2}-\frac{1}{2} & k / 2 \\
0 & -0 & 0 & \cdots & 0 & 0 & 0-0
\end{array}
$$

Fig. 18. Dynkin-like representation of the $\mathrm{SU}(2)$ fusion rules.

with one of the dots in the Dynkin diagram of $A_{k+1}$ as in Fig. 18.

The lines joining different dots represent the result of performing the OPE with the spin $1 / 2$ field. Following the rules of this diagram it is very easy to reconstruct the Bratelli diagram for any level. In cases where the operator algebra is more complicated, we will get multi-dimensional Dynkin-like diagrams. In the minimal models for example we have two generators. $\phi_{(2,1)}, \phi_{(1,2)}$ of the operator algebra leading to two-dimensional graphs and associated Bratelli towers. Other examples of fusion rules will be analyzed in later sections. In our analysis of quantum groups we will show that the fusion rules are a consequence of the properties of tensor products of representations. Before concluding this subsection we would like to add that the braiding and fusing matrices can be obtained in principle by solving the Knizhnik-Zamalodchikov equations (4.28). This is unfortunately a very cumbersome procedure. We will show later that the representation theory of quantum groups provides explicit formulae to compute the duality matrices from simple group theoretic manipulations.

\subsection{Partition functions. modular invariants}

For the minimal theories the partition function is built out from the characters of the Virasoro algebra. For WZW models we can also construct the partition functions in terms of the Kac-Moody characters. So far we have ignored the antiholomorphic dependence of the primary fields. The physical spectrum of the theory however will contain operators with representation $(R, \bar{R}), R(\bar{R})$ with respect the holomorphic (antiholomorphic) Kac-Moody algebra. To find the physical spectrum in a theory where the left and right chiral algebras are isomorphic we have to determine the possible modular (and monodromy) invariant combinations of left and right characters $^{51}$ :

$$
\begin{aligned}
\chi_{R}(\tau) & =e^{2 \pi i \tau\left(\frac{c_{R}}{2 k+c_{G}}-\frac{k d i m G}{13\left(2 k+c_{G}\right)}\right)} \operatorname{tr}_{R} e^{2 \pi i N} \\
& =\operatorname{tr}_{R} e^{2 \pi i\left(L_{0}-c / 24\right)}
\end{aligned}
$$

(and similarly for $\left.\chi_{\breve{R}}(7)\right)$. In $(4.68) N$ is the "number" operator. The partition function takes the form:

$$
Z=\sum_{(R, \bar{R})} N_{R \bar{R}} \chi_{R}(\tau) \overline{\chi_{\bar{R}}(\tau)}
$$

(we are assuming for simplicity that $G$ is a simple group, the arguments are easily extended to the general case). Since under $\tau \rightarrow \tau+1 \chi_{R}(\tau)$ changes by a phase, the first requirement on $N_{R \bar{R}}$ is that

$$
N_{R R}=0 \quad \text { unless } \quad h_{R}-h_{R} \in \mathbb{Z} \text {. }
$$

Invariance under $S: \tau \rightarrow-1 / \tau$ of $Z$ is more complicated and we need to know the modular properties of the character (or as we will show in the next section the fusion rules for the fields in the theory). The Kac-Moody ${ }^{49}$ and minimal model characters ${ }^{10}$ can all be expressed in terms of level $N$ $\vartheta$-functions. The simplest ones are:

$$
\vartheta_{n, k}(z, \tau ; n)=e^{-2 \phi_{i} k_{u}} \sum_{j \in \mathbb{Z}+n / 2 k} e^{2 \pi i k\left(j_{r}^{2}-j z\right)} n \in \mathbb{Z} / 2 k .
$$

Using Poisson resummation one shows that:

$$
\vartheta_{n, k}\left(-\frac{z}{\tau},-\frac{1}{\tau}, u+\frac{z_{2}}{4 \tau}\right)=\left(-\frac{i \tau}{4 k}\right)^{1 / 2} \sum_{e \in Z / 2 k Z} e^{i \pi e n / k} \vartheta_{e, k}(z, \tau ; u) .
$$

Furthermore:

$$
\vartheta_{n, k}(z, z+1 ; u)=e^{i \pi n^{2} / 2 k} \vartheta_{n, k}(z, \tau ; u)
$$

The Rocha-Caridi characters (3.49) with the change of notation:

$$
c=1-6 \frac{(r-s)^{2}}{r s}
$$

$r, s$ coprime $(r, s)=1$ becomes:

$$
\begin{aligned}
& \chi_{p, q}(\tau)=\frac{1}{\eta(\tau)}\left[\vartheta_{n_{-}, k}(0, \tau ; 0)-\vartheta_{n_{+}, k}(0, \tau ; 0)\right] \\
& \eta(\tau) \quad=q^{1 / 24} \prod_{n=1}^{\infty}\left(1-q^{n}\right), q=e^{2 \pi i \tau} \\
& k \quad=r s \quad n_{ \pm}=r p \pm s q .
\end{aligned}
$$


Since $(r, s)=1,0<p<r, 0<q<s$ using (4.72) and some simple arithmetic properties one can show that

$$
\begin{aligned}
\chi_{p, q}(\tau+1)= & e^{i \pi n_{-}^{2} / 2 k} \chi_{p, q}(\tau) \\
\chi_{p, q}\left(-\frac{1}{\tau}\right)= & -2 \sqrt{\frac{2}{k}} \sum_{p^{\prime}, q^{\prime}}(-1)^{p^{\prime} q+p q^{\prime}} \\
& \sin \left(\frac{n s}{s} p p^{\prime}\right) \sin \left(\frac{n s}{r} q q^{\prime}\right) \chi_{p^{\prime} q^{\prime}}(\tau) .
\end{aligned}
$$

For Kac-Moody algebras the characters $(4.68)$ are also known ${ }^{49}$. For instance, in the SU(2) algebra at level $k$ the character for the spin $j$ representation is:

$$
\chi_{j}(\tau)=\frac{\vartheta_{-2 j-1, k+2}-\vartheta_{2 j+1, k+2}}{\vartheta_{-1,2}-\vartheta_{1,2}} \quad(0, \tau ; 0)
$$

this formula is intended as a limit since both numerator and denominator vanish. Using the identity:

$$
\eta^{3}(\tau)=\sum_{m \in \mathbb{Z}}(4 m+1) q^{\frac{1}{8}(4 m+1)^{2}}
$$

one shows that

$$
\begin{aligned}
& \chi_{j}(\tau)=\eta^{-3}(\tau) \sum_{m \in Z}^{\left(2_{j+1}+m n\right)} q^{\left(N_{m}+2 j\right)^{2} / 2 N} \\
& N \quad=2(k+2)
\end{aligned}
$$

although in the computation of modular transformations it is more convenient to use the representation (4.76). From (4.72) we obtain:

$$
\begin{aligned}
& \chi_{j}(-1 / \tau)=\sqrt{\frac{2}{k+2} \sum_{j^{\prime}} \sin \frac{\pi(2 j+1)\left(2 j^{\prime}+1\right)}{k+2} \chi_{j^{\prime}}(\tau)} \\
& \chi_{j}(\tau+1)=e^{i \pi\left(\frac{(2 j+1)^{3}}{2(k+2)}-\frac{1}{\imath}\right)} \chi_{j}(\tau) .
\end{aligned}
$$

For SU(2) and the minimal models Capelli Itzykson and Zuber ${ }^{52}$ have. given the complete classification of modular invariant combinations of the characters. From the remarks following (4.61) we know that the fusion rules for the minimal models follow from those of $\mathrm{SU}(2) p \times \mathrm{SU}(2) p^{t}$ with $r=p+2 s=p^{\prime}+2$, thus it is reasonable to expect that once the modular invariants for $\mathrm{SU}(2)$ are known, we can also construct all the modular invariants for the minimal models. This is indeed the case as shown in Ref. 52 .

Using simple algebra it is easy to show that the matrix

$$
S_{j j^{\prime}}=\sqrt{\frac{2}{k+2}} \sin \pi \frac{(2 j+1)\left(2 j^{\prime}+1\right)}{k+2} \quad 0 \leq j, j^{\prime} \leq k / 2
$$

is orthogonal. Hence $S, T(4.78)$ defines a unitary representation of the modular group $\mathrm{PSL}_{2}(\mathbb{Z})$ acting on the characters. Hence, for every level $k$ we can always construct the diagonal or $A$ invariant:

$$
Z(\tau, \bar{\tau})=\sum_{j=0}^{k / 2}\left|\chi_{j}(\tau)\right|^{2}
$$

The complete classification is intimately connected with the A-D-E classification of simple Lie algebras. To exhibit this relation more clearly we label the characters $\chi_{j}$ as $\chi_{2 j+1}(\tau)$. The results of Ref. 52 are:

$$
\begin{aligned}
& k \geq 1 \sum_{\lambda=1}^{k+1}\left|\chi_{\lambda}\right|^{2} \\
& k=4 \rho, \rho \geq 1, \sum_{\substack{\lambda \text { odd }=1 \\
\lambda \neq 2 \rho+1}}^{4 \rho+1}\left|\chi_{\lambda}\right|^{2}+\left|\chi_{2 \rho}\right|^{2}+\sum_{\lambda \text { odd }=1}^{2 \rho-2}\left(\chi_{\lambda} \chi_{4 \rho+2-\lambda}^{*}+\text { c.c. }\right) \\
& k=4 \rho-2, p \geq 2 \sum_{\chi_{\text {odd }}=1}^{4 \rho-1}\left|\chi_{\lambda}\right|^{2}+\left|\chi_{2 \rho}\right|^{2}+\sum_{\lambda \text { even=2 }}^{2 \rho-2}\left(\chi_{\lambda} \chi_{4 \rho-\lambda}^{*}+\text { c.c. }\right) \\
& k+2=12\left|\chi_{1}+\chi_{7}\right|^{2}+\left|\chi_{4}+\chi_{8}\right|^{2}+\left|\chi_{5}+\chi_{11}\right|^{2} \\
& k+2=18\left|\chi_{1}+\chi_{17}\right|^{2}+\left|\chi_{5}+\chi_{13}\right|^{2}+\left|\chi_{7}+\chi_{11}\right|^{2}+\left|\chi_{9}\right|^{2} \\
& k+2=30\left|\chi_{1}+\chi_{11}+\chi_{19}+\chi_{29}\right|^{2}+\left|\chi_{7}+\chi_{13}+\chi_{17}+\chi_{23}\right|^{2}
\end{aligned}
$$


Note that the values of $\lambda$ for each invariant coincide with the exponents of the simply-laced algebras on the right-hand side of (4.81). The connection between these invariants, the simply laced algebras, and the crytallographic subgroups of $\mathrm{SU}(2)$ is not well-understood. The nondiagonal invariants in (4.81) can be interpreted in a variety of ways. For example the $D$-invariants $D_{2 \rho+2}$ can be thought of a WZW models on $\mathrm{SO}(3)=\mathrm{SU}(2) / Z_{2}^{13}$. The possibility of having nondiagonal invariants is also related to the existence of a bigger underlying chiral algebra or that there is a nontrivial automorphism of the fusion rules ${ }^{53,17}$. Consider for example $\mathrm{SU}(2)_{4 k}$, the primary fields have spin $j=0,1 / 2,1, \ldots, 2 k$ and from the fusion rules (4.61) we know that

$$
\begin{aligned}
& \phi_{2 k} \times \phi_{2 k}=1 \\
& \phi_{2 k} \times \phi_{j}=\phi_{|2 k-j|} .
\end{aligned}
$$

Furthermore the braiding properties of $\phi_{j}$ and $\phi_{|2 k-j|}$ are the same as long as $j$ is an integral. The field $\phi_{2 k}$ extends the chiral algebra (the Kac-Moody algebra) with a field of dimension $k$. If $k=1, \mathrm{SU}(2)_{1}$ algebra ${ }^{54}$. Using the extension of the chiral algebra we see that $\chi_{j}$ and $\chi_{2 k-j}$ pair up into a single module of the extended algebra for $j \neq k$. Rewriting the $k=4 \rho$ invariant (4.81) as:

$$
\sum_{\substack{j \text { integer } \\ j \neq \rho}}\left|\chi_{j}+\chi_{2 \rho-j}\right|^{2}+2\left|\chi_{\rho}\right|^{2}
$$

we see that the characters of the extended algebra are of the form $\chi_{j}+\chi_{2 \rho-j}$. (The $E_{8}$ invariant can be identified with the $G_{2}$ Kac-Moody algebra at level $1^{54}$ and the $E_{6}$ invariant with $S_{p}(4)$ level 1 ).

Out of the invariants (4.81) we can construct the modular invariant combinations of the minimal models. Write $c=1-6\left(p-p^{\prime}\right)^{2} / p p^{\prime},\left(p, p^{\prime}\right)=1$ and $k=p-2, k^{\prime}=p^{\prime}-2$. Since $p, p^{\prime}$ are coprime, they cannot be both even, hence one of the SU(2) modular invariants must be of A-type. The complete set is 52 :

$$
\begin{aligned}
\frac{1}{2} \sum_{r=1}^{p^{\prime}-1} \sum_{s=1}^{p-1}\left|\chi_{r s}\right|^{2} & \left(A_{p^{\prime}-1}, A_{p-1}\right) \\
p^{\prime}=4 \rho+1 & \frac{1}{2} \sum_{s=1}^{p-1}\left\{\sum_{\substack{r+d d=1 \\
r \neq 2 \rho+1}}^{4 \rho+1}\left|\chi_{r s}\right|^{2}+2\left|\chi_{2 \rho+1, s}\right|^{2}\right.
\end{aligned}
$$

$$
\left.+\sum_{r o d d=1}^{2 p-1}\left(\chi_{r \cdot} \chi_{r, p-s}^{*}+c . c .\right)\right\}
$$$$
\left(D_{2 \rho+2}, A_{p-1}\right)
$$$$
{\underset{p}{p \geq 2}}_{p=4 \rho}^{\prime} \quad \frac{1}{2} \sum_{s=1}^{p-1}\left\{\sum_{\text {rodd }=1}^{4 \rho-1}\left|\chi_{\mathrm{rs}}\right|^{2}+\left|\chi_{2 \rho, s}\right|^{2}\right.
$$$$
\left.+\sum_{\text {reven }=1}^{2 p-2}\left(\chi_{r s} \chi_{p^{\prime}-r, s}^{*}+\text { c.c. }\right)\right\}
$$

$$
p^{\prime}=12 \frac{1}{2} \sum_{s=1}^{p-1}\left\{\left|\chi_{i s}+\chi_{7 s}\right|^{2}+\left|\chi_{4 s}+\chi_{8 s}\right|^{2}+\left|\chi_{5 s}+c h i_{1 i_{s}}\right|^{2}\right\}
$$

$$
\begin{aligned}
p^{\prime}=18 \frac{1}{2} \sum_{s=1}^{p-1}\left\{\left|\chi_{1 s}+\chi_{17 s}\right|^{2}+\left|\chi_{5 s}+\chi_{13 s}\right|^{2}\right. \\
+\left|\chi_{7 s}+\chi_{11 s}\right|^{2}+\left|\chi_{9 s}\right|^{2}+\left[\left(\chi_{3 s}+\chi_{15 s}\right) \chi_{9 s}+\text { c.c. }\right]
\end{aligned}
$$

$$
\begin{aligned}
p^{\prime}=30 \frac{1}{2} \sum_{s=1}^{p-1} & \left\{\left|\chi_{1 s}+\chi_{11 s}+\chi_{19 s}+\chi_{29 s}\right|^{2}\right. \\
& \left.+\left|\chi_{7 s}+\chi_{13 s}+\chi_{17 s}+\chi_{23 s}\right|^{2}\right\}
\end{aligned}
$$

The character formulae for other Kac-Moody algebras is known, and it is easy to show that the diagonal combination of left and right character is always modular invariant. Furthermore, in analogy with $\mathrm{SU}(2)$, if $H \subset G$ is a subgroup of the center of $G, H \subset Z(G)$, we can construct the $G / H$ WZW-model using orbifold techniques (as in Ref. 13) to obtain analogues of the $D$-series. Unfortunately it is not known how to classify all possible modular invariant combinations and whether it has something to do with finite crystallographic subgroups of $G$. (for some recent progress and references to the literature, see Refs. 55 and 56 ).

This concludes our quick survey of conformal theories with Kac-Moody symmetry. It is interesting to notice that each module of $\hat{G}_{k}$ consists of an 
infinite number of Virasoro modules ( $G$ simple). The notion of rationality of a CFT depends on the chiral algebra underlying the theory. From this point of view the classification of rational theories RCFT translates into the classification of chiral algebras and the construction of their associated minimal series.

\section{Fusion Algebras. The Verlinde Conjecture \\ 5.1 The fusion algebra and its properties}

In this section we would like to review in some details the properties of this fusion algebra. The unexpected connection between the fusion rules and the modular transformation properties of the genus 1 characters ${ }^{15}$ has generated a lot of work in the classification of rational CFT (RCFT) $16,17,18,22,23,24,25$.

Generically, a RCFT is characterized by a chiral algebra $\sigma=\sigma_{L} \otimes$ $\sigma_{R}$ and a physical Hilbert space $\mathcal{K}$ which splits into a finite number of irreducible representation (irreps) of $\sigma_{L} \otimes \sigma_{R}: \mathcal{H}=\oplus_{(i, \bar{I})} \mathcal{H}_{i} \otimes \mathcal{K}_{i}$ where $\mathcal{K}_{i}$ (respectively $\mathcal{H}_{\bar{I}}$ ) is an irrep. of $\sigma_{L}$ (respectively $\sigma_{R}$ ). The primary fields generating $\mathfrak{X}_{i} \otimes \mathcal{K}_{\mathfrak{i}}$ are denoted by $\phi_{h, \bar{h}}$. If we consider only the left moves, the OPE yield the fusion rules:

$$
\left[\phi_{i}\right] \times\left[\phi_{j}\right]=N_{i j}^{k}\left[\phi_{k}\right] .
$$

The numbers $N_{i j}^{k}$ are either zero or positive integers - Defining the matrices $\left(N_{i}\right)_{j}^{k}=N_{i j}^{k}$ the associativity of the OPE implies that:

$$
\left[N_{i}, N_{j}\right]=0 \text {. }
$$

It is obvious from (5.1) that $N_{i j}^{k}=N_{j i}^{k}$, hence to every set of fusion rules we can associate a commutative and associative algebra with unit:

$$
x_{i} x_{j}=N_{i j}^{k} x_{k} \quad i, j, k=0,1, \ldots, N-1 .
$$

Since the number of primary operators (or conformal families) is finite $\phi_{0}, \ldots, \phi_{N-1}$, for every $\phi_{i}$ we can construct a conjugate field $\phi_{T}$ such that the OPE $\phi_{i} \times \phi_{\tau}$ contains the identity. The SU(2) examples and minimal models studied in the previous sections are all examples of self-conjugate representations. The "charge conjugation" matrix $C: \phi_{i} \rightarrow \phi_{\bar{i}}$ is given by particular,

$$
N_{i j k}=N_{i j}^{l} C_{l k}
$$

is a totally symmetric symbol. In

$$
N_{i j}^{l} N_{m l}^{s}=N_{m j}^{l} N_{i l}^{s}
$$

simply substitute $s=0$, then:

$$
N_{i j m}=N_{m j i}
$$

which together with $N_{i j}^{k}=N_{i j}^{k}$ implies the desired property. We can analyze the representation theory of the algebra (5.3) using the techniques of finite group theory. First, the existence of a nondegenerate charge conjugation matrix implies that the fusion algebra (5.3) is semi-simple, hence the regular representation $x_{i} \rightarrow N_{i}$ is both reducible and fully reducible, and there are $N$ one-dimensional inequivalent irreducible representations. Since all the $N_{i}$ commute, they can be diagonalized simultaneously, and the onedimensional irreps can be labelled by these eigenvalues:

$$
\begin{aligned}
& N_{i} \sim\left(\begin{array}{lll}
\lambda_{i}^{(0)} & & \\
& \ddots & \\
& & \lambda_{i}^{(N-1)}
\end{array}\right) \\
& \lambda_{i}^{(\alpha)} \lambda_{j}^{(\alpha)}=N_{i j}^{k} \lambda_{k}^{(\alpha)}
\end{aligned}
$$

The matrices $N_{i j}^{k}$ are quite useful in determining the dimension of the conformal blocks of a given theory on an arbitrary Riemann surface. For instance the space of 4-point blocks of the type described in Fig. 19 has dimensions:

$$
\sum_{p} N_{i j}^{p} N_{k p}^{l}=\left(N_{j} N_{k}\right)_{i}^{l} .
$$

The associativity of (5.3) implies that dimension formulae like the one in (5.6) are independent of the skeleton graph (Fig. 19) we use to compute it. In particular, we can compute the dimension of the space of conformal characters on a Riemann surface of genus $g$. If $M_{g, o}$ is the moduli space of genus $g$ surface without distinguished points, the characters are sections of a (projectively) flat holomorphic vector bundle over $M_{g, 0}$. We can use the skeleton in Fig. 20 to compute the dimension of this vector bundle. This figure looks like two-point function on a genus $r$ surface. The dimension for fixed $i$ is easily computed to be

$$
\sum_{\substack{k_{1} \ldots . \\ j_{1} \ldots \\ l_{1} \ldots}} N_{i k_{1}}^{j_{1}} N_{j_{1} k_{1}}^{l_{1}} N_{l_{2} k_{2}}^{j_{2}} N_{j_{3} k_{3}}^{l_{2}} \ldots N_{l_{r-1} k_{r}}^{j_{r}} N_{j_{r} k_{r}}^{i}=\sum_{k_{2} \ldots k_{r}}\left(N_{k_{1}}^{2} \ldots N_{k_{r}}^{2}\right)_{i}^{i}
$$




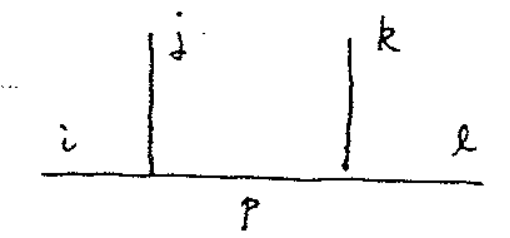

Fig. 19. Four point block.

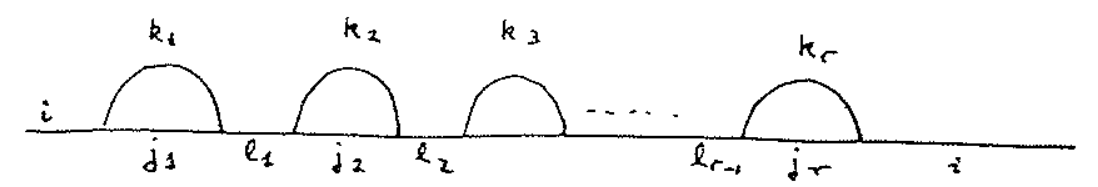

Fig. 20. Skeleton diagram to compute the number of generalized characters at genus $g$.

we close the loop by summing over $i$. Hence for a germs $g$ surface, the number of generalized characters is:

$$
\operatorname{dim} V_{g}=\operatorname{Tr}\left(\sum_{k=0}^{n-1} N_{k}^{2}\right)^{g-1}
$$

At genus 1 we have the standard Virasoro characters:

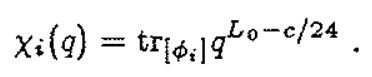

Under modular transformations:

$$
\begin{aligned}
T: \chi_{i} \rightarrow \chi_{i}\left(q e^{2 \pi i}\right) & =e^{2 \pi i\left(h_{i}-c / 24\right)} \chi_{i}(q) \\
S: \quad \chi_{i} \rightarrow \chi_{i}(-1 / \tau) & =S_{i}^{j} \chi_{j}(\tau) .
\end{aligned}
$$

$T$ and $S$ generate PSL $2(\mathbb{Z})$. If the charge conjugation matrix is not then $S^{2}=(S T)^{3}=C$. In a torus as in Fig. 21 the modular transformation $\tau \rightarrow$ $-1 / \tau$ acts on the cycles as $(a, b) \rightarrow(-b, a)$. Hence $S^{2}:(a, b) \rightarrow(-a,-b)$ equivalent to charge conjugation. Since $C^{2}=1, S^{4}=(S T)^{6}=1$ for the representation of $S, T$ furnished by the CFT. In [15] E. Verlinde introduced a set of operator $\phi_{i}(a), \phi_{i}(b)$ defined as follows: $\phi_{i}(a)$ acts on the characters by first inserting the identity factorized as $\phi_{i} \times \phi_{\tau}$, then we transport the field $\phi_{i}$ around the a cycle, and combine it with $\phi_{i}$ into the identity again. Similarly for $\phi_{i}(b)$ around the $b$-cycle. If we think of the $a$-cycle as space and the $b$ cycle as time, $\phi_{i}(a)$ acts diagonally on the characters:

$$
\phi_{i}(a) \chi_{j}=\lambda_{i}^{(j)} \chi_{j}
$$

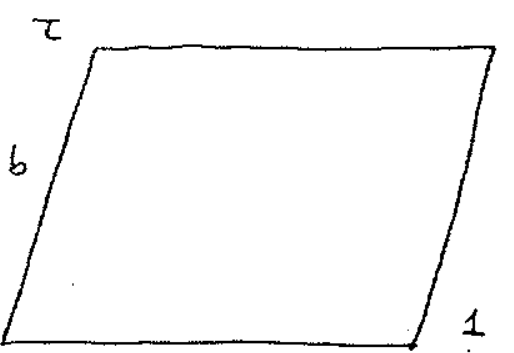

Fig. 21. Fundamental region for a torus $\mathbb{C} /\{z+r z\}$.

(for the time being the $\lambda_{i}^{(j)}$ are different from the eigenvalues (5.5) although they will be shown to be the same). The operator $\phi_{i}(b)$ has a less trivial action on the characters. If we start with the character of the identity $\phi_{0}(\tau)$ it is clear that the action of $\phi_{i}(b)$ generates $\chi_{i}(\tau): \phi_{i}(b) \chi_{0}(\tau)=\chi_{i}(\tau)$ and this also defines the normalization of $\phi_{i}(b)$. Acting on other characters we will have:

$$
\phi_{i}(b) \chi_{j}(\tau)=A_{i j}^{k} \chi_{k}(\tau)
$$

The constants $A_{i j}^{k}$ satisfy $A_{i j}^{k}$, and they also define an associative algebra as a consequence of the associativity of the OPE. By looking at many examples and using some intuitive arguments, E. Verlinde ${ }^{15}$ conjectured that $A_{i j}^{k}=$ $N_{i j}^{k}$. Later G. Moore and N. Seiberg ${ }^{16}$ proved this conjecture for RCFT using the pentagon equation (Fig. 5). Before presenting the proof, we will explore its consequences. First of all, notice that the fact that $S$ acts on the characters implies that there is some notion of completeness of the operator algebra in the CFT. Working at tree level one may satisfy all consistency conditions regarding monodromy invariance etc with a smaller set of fields. For instance in the minimal series we can consider the thermal subalgebra of operators $\left\{\phi_{(1, m)}\right\}$. This family closes under operator products and it satisfies all the requirements of locality and duality. One finds that some of the fields are missing when one implements the modular transformation $\tau \rightarrow$ $-1 / \tau$. It is still an open question whether the requisite on the characters to provide a representation of the modular group can be translated into some set of completeness conditions for the operator algebra at genus zero. However once (5.10) is satisfied and we assume $N_{i j}^{k}=A_{i j}^{k}$ we immediately conclude that the matrix $S_{i}^{j}$ diagonalizes the fusion matrices $N_{i}$. Since the operator $S$ exchanges the $a$ and $b$ cycles we have that:

$$
\phi_{i}(b)=S^{-1} \phi_{i}(a) S
$$


as operators on characters. Therefore

$$
N_{i}=S D_{i} S^{-1} \quad D_{i}=\left(\begin{array}{ccc}
\lambda_{i}^{(0)} & & \\
& \ddots & \\
& & \lambda_{i}^{(N-1)}
\end{array}\right) \text {. }
$$

From $N_{i} S=S D_{i}$ and $N_{i}^{j}=\delta_{i}^{j}$ we obtain:

$$
\lambda_{i}^{(j)}=S_{i}^{j} / S_{0}^{j}
$$

and:

$$
N_{i j}^{k}=\sum_{m} S_{j}^{m} \frac{S_{i}^{m}}{S_{0}^{m}}\left(S^{-1}\right)_{m}^{k}
$$

since $S^{2}=C, S^{-1}=S C, C^{2}=1$ we obtain:

$$
N_{i j k}=\sum_{m} \frac{S_{i}^{m} S_{j}^{m}}{S_{0}^{m}} S_{m}^{k} .
$$

it is clear that if $S$ is symmetric, $N$ is fully symmetric in $(i, j, k)$. The converse is true. Take $k=0$ in $(5.16)$

$$
N_{i j 0}=C_{i j}=\sum_{m} S_{i}^{m} S_{j}^{m} \frac{S_{m}^{0}}{S_{0}^{m}}=\sum_{m} S_{i}^{m} S_{m}^{j} .
$$

Hence

$$
S_{m}^{j}=S_{j}^{m} \frac{S_{m}^{0}}{S_{0}^{m}}
$$

Taking $m=j$ we learn that $S_{0}^{j}=S_{j}^{0}$ and therefore

$$
S^{T}=S
$$

Finally it is also possible to prove the unitarity of $S$. This is equivalent to showing that $S C=S^{*}$. The charge conjugation symmetry of the fusion rules $N_{i j}^{\bar{k}}=N_{i j}^{k}$ implies that the eigenvalues of $N_{r}$ are $\lambda_{\tau}^{(j)}=\lambda_{i}^{(j) *}$. Then:

$$
S_{i}^{j} / S_{0}^{j}=S^{* j} j / S_{0}^{* j} \text {. }
$$

Since $S_{\tau}^{j}=(C S)_{i}^{j}$, taking $j=0$ and using $C S=S C$ we obtain $\left(S_{i}^{0}\right)^{*} / S_{i}^{0}=$

$$
(C S)_{i}^{j} \frac{\left(S_{0}^{0}\right)^{*}}{S_{0}^{0}}=\left(S_{i}^{j}\right)^{*}
$$

Using $S^{* 2}=C=S^{2}$ we obtain $\left(S_{0}^{0}\right)^{*} / S_{0}^{0}= \pm 1$. To fix the sign we can use $\tau=i$ as a fixed point of the transformation $S: \tau \rightarrow-1 / \tau \mathrm{S}$. Also at $\tau=i$ the characters are all real $\left(\chi_{i}(\sqrt{-1})=v_{i}\right)$, hence $S$ has an eigenvalue equal to one with a real eigenvector: $S_{i}^{j} v_{j}=v_{i}$. For $i=0, S_{0}^{i} v_{i}=v_{0}$. Since $\left(S_{0}^{i}\right)^{*}= \pm S_{0}^{i}$, taking complex conjugates in $S_{0}^{i} v_{i}=v_{0} \quad v_{i}^{*}=v_{i}$ we avoid a contradiction only if the plus sign is chosen. Thus

$$
S_{0}^{i}=\left(S_{0}^{i}\right)^{*} \text { and } S_{i}^{j *}=(C S)_{i}^{j}
$$

we can summarize all the properties of the fusion algebra proved so far:

$$
\begin{aligned}
& S^{*}=C S=S C \\
& S^{T}=S, S^{2}=C \\
& N_{i j k}=\sum_{m} \frac{S_{i}^{m} S_{j}^{m} S_{m}^{k}}{S_{0}^{m}} .
\end{aligned}
$$

These conditions, specially the symmetry of $S$ are surprisingly stringent. It is very easy to construct many examples of associative and commutative algebras (5.3) where structure constants are positive integers, for example the group algebras associated to finite groups. However the symmetry conditions on $S$ rule not almost all examples. If the finite group is Abelian we automatically have a solution as a product $Z_{N_{2}} \times Z_{N_{2}} \times \ldots \times Z_{N_{m}}$ where $\mathbf{Z}_{p}$ is the group of integers module $p$. For a single factor $\mathbf{Z}_{N}$, the rational Gaussian models ${ }^{5}$ exhibit a set of fusion rules according to the representation theory of $Z_{N}$. The primary fields are labelled by an integer $\bmod N_{;} \phi_{p}$ and the fusion rules are:

$$
\phi_{p} \times \phi_{p^{\prime}}=\phi_{p+p^{\prime}} \quad(\bmod N)
$$

For this theory:

$$
\begin{aligned}
(S \chi)_{p} & =\frac{1}{\sqrt{N}} \sum_{p^{\prime}} e^{2 \pi i p p^{\prime} / N} \chi_{p^{\prime}} \\
S_{p p^{\prime}} & =\frac{1}{\sqrt{N}} e^{2 \pi i p p^{\prime} / N}
\end{aligned}
$$

and the modular transformation $S$ is equivalent to finite Fourier transform; then:

$$
\lambda_{p}^{\left(p^{\prime}\right)}=e^{2 \pi i p p^{\prime} / N} \quad C_{p p^{\prime}}=\delta_{p+p^{\prime}, 0} \quad(\bmod N) .
$$


As pointed out at the beginning of this section, we can analyze (5.3) using the techniques of the representation theory of finite groups. The regular representation $\phi_{i} \rightarrow N_{i}$ is fully reducible, and we can derive the orthogo nality relations for the characters. The characters of the $n$ one-dimensional representations of (5.3) are:

$$
\lambda^{(j)}\left(x_{j}\right)=\lambda_{i}^{(j)} \equiv \lambda_{j}^{(i)}
$$

given the matrix $\Lambda, \Lambda_{i j}=\lambda^{(i)}\left(x_{j}\right)$ let $N, N_{i j}=n_{j}^{(i)}=n_{i}(j)$ be its inverse. Then the orthogonality properties of the characters are ${ }^{59}$ :

$$
\begin{aligned}
& \sum_{k} \lambda_{i}(k) \overline{\lambda_{j}(k)}=\frac{1}{\pi_{0}(i)} \delta_{i j} \\
& \sum_{k} n_{0}(k) \lambda_{k}(i) \overline{\lambda_{k}(j)}=\delta_{i j} \\
& \overline{\lambda_{j}(k)}=\lambda_{j}(\vec{k}) .
\end{aligned}
$$

The fusion matrices $N_{i}$ are diagonalized now by the matrix

$$
S_{i j}=\sqrt{n_{0}(j)} \lambda_{j}(i)
$$

If $S$ is symmetric (as required by (5.18)) then from (5.20) one easily derives $S^{2}=C$, where $C$ is defined as in CFT.

\subsection{Proof of the Verlinde conjecture}

We now present the proof of $A_{i j}^{k}=N_{i j}^{k}$ using the pentagon equation. ${ }^{16.53}$ In Sec. 7 we will describe a proof of this conjecture using the properties of quantum groups. The coefficient $N_{i j}^{k}$ label the number of possible couplings between the conformal families $i, j, k$. The three-point block can be represented as in Fig. 22 with the extra label counting all possible couplings. In order to avoid making the notation very complicated, we will consider self-conjugate theories and concentrate on one coefficient $N_{i j}^{k}$. The arguments are easily generalized to include complex representations. The operator $\phi_{i}(b)$ is defined as a sequence of manipulations on the chiral blocks of the two-point function on the torus. These operations appear in Fig. 23.
The moves $A, B$ can be written in terms of the $F, B$ moves in Fig. 4.

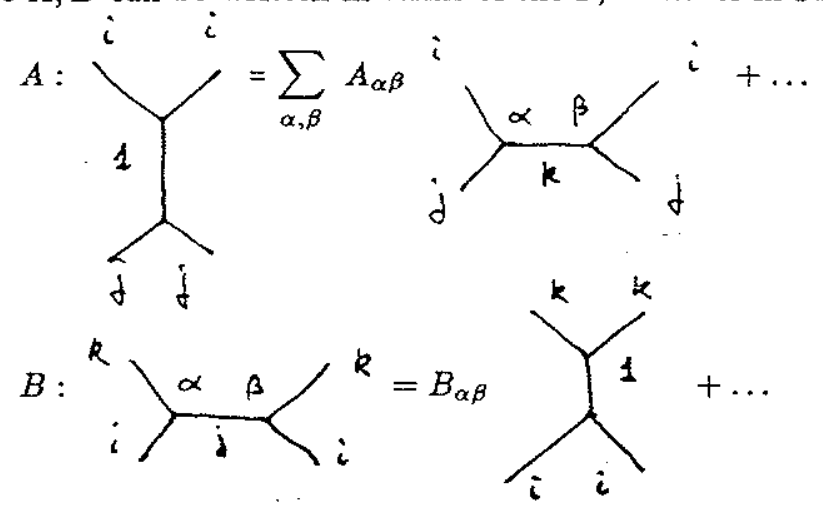

$$
\alpha, \beta=1, \ldots, N_{i j}^{k}
$$

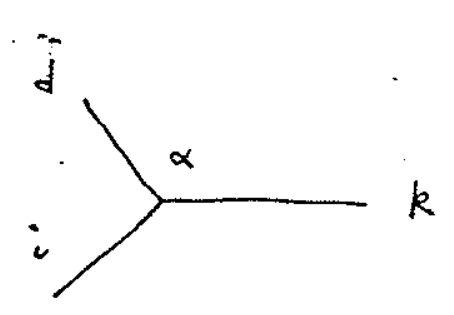

Fig. 22. An extra label $\alpha$ is unnecessary when $N_{i j}^{k} \neq 1$.

$$
\alpha=1,2, \ldots N_{i j}^{k}
$$
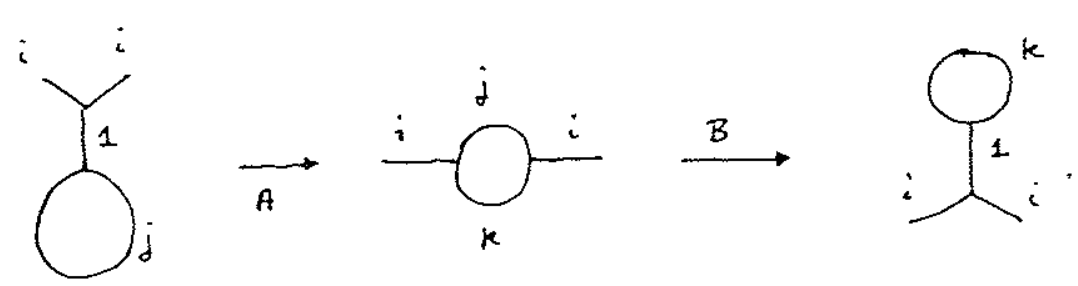

Fig. 23. Operators involved in the definition of the Verlinde operators.

To normalize $\phi_{i}(b)$ we will also need:

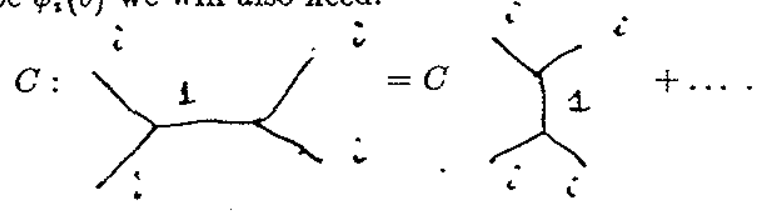


In $F, B$ notation:

$$
\begin{aligned}
& C=F_{11}\left[\begin{array}{ll}
i & i \\
i & i
\end{array}\right] \\
& B_{\alpha \beta}=F_{j 1}\left[\begin{array}{ll}
k & k \\
i & i
\end{array}\right]_{\alpha \beta} \\
& A_{\alpha \beta}=F_{1 k}\left[\begin{array}{ll}
j & i \\
j & i
\end{array}\right]^{\alpha \beta} .
\end{aligned}
$$

The dots in (5.21) and (5.22) are the contributions from other representations and they will not be important for the argument. To prove Verlinde's conjecture, we have to show that

$$
\sum_{\alpha, \beta=1}^{N_{i j}^{k}} \frac{A_{\alpha \beta} B_{\alpha \beta}}{c}=N_{i j}^{k}
$$

according to the moves in Fig. 23. Using the associativity moves which generate the pentagon identity Fig. 5 we obtain the following identity

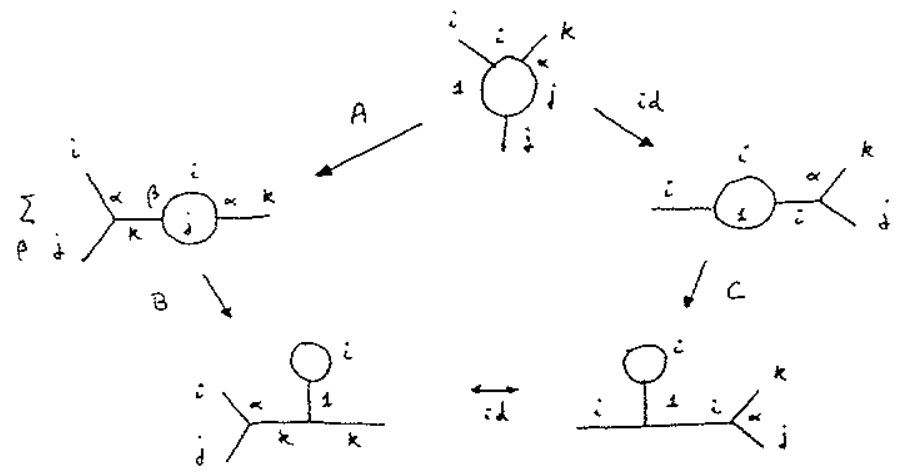

Equations (5.25) become:

$$
\sum_{\beta=1}^{N_{i j k}} A_{\alpha \beta} B_{\alpha \beta}=c .
$$

Since $C$ is independent of $\alpha, \beta$ we finally obtain (5.24).
As we will see in later sections, the connection between CFT and knot and link invariants follows from the fusion algebra and its properties (5.18). Most of the topological underpinnings of RCFT seem to stem from (5.18).

\subsection{Constraints from rationality}

While we are analyzing general properties of RCFT, it is useful to explore the constraints on the conformal dimension in a RCFT after the fusion rules are known. Using very simple arguments it is possible to show that some fusion rules are not allowed by modular invariance. More important, one can prove the rationality of all the conformal dimensions and the value of $c$ for a RCFT ${ }^{19,20}$. As argued in Sec. 2, the mapping class group of the sphere with $n$ distinguished points can be described in terms of an extension of the braid group $B_{n}$ by the Dehn twists around the points $p_{i}$ with local parameters $Z_{i}, Z_{i}\left(P_{i}\right)=0$. Together with the standard defining properties of the braid group we have the relations (2.68). These relations together with (2.69) can be used to derive the relation (2.70). To exhibit the constraints on the possible conformal dimensions, as a consequence of these relations, let us consider an example. We assume that the RCFT has only three primary fields $1, \varepsilon, \sigma$ with fusion rules

$$
\begin{gathered}
\varepsilon \cdot \varepsilon=1 \quad \sigma \cdot \sigma=1+\varepsilon \\
\varepsilon \cdot \sigma=\sigma .
\end{gathered}
$$

We can analyze different conformal blocks depending on the fields $\varepsilon, \sigma$ assigned to the four points $p_{1}, P_{2}, P_{3}, P_{4}: \varepsilon \varepsilon \varepsilon \varepsilon, \sigma \sigma \sigma \sigma, \varepsilon \varepsilon \sigma \sigma$. The dimension of the space of blocks $\varepsilon \varepsilon \varepsilon \varepsilon$ is 1 , and the eigenvalues of $T_{12}$, depend only on the dimensions of the fields appearing in the OPE of the fields at $p_{1}$ and $p_{2}$. The Dehn twist $\tau_{12}$ is, in this basis, equivalent to exp $2 \pi i L_{0}$ on the intermediate channels. Since $\varepsilon \cdot \varepsilon=1, \tau_{i j}$ are $1 \times 1$ matrices with eigenvalues 1. Hence we learn that

$$
\alpha_{4}^{4}=1 \quad \alpha_{i}=e^{2 \pi i h_{i}} .
$$

With $\sigma \sigma \sigma \sigma$, the block has dimension 2, and the eigenvalues of $\tau_{i j}$ are $1, \alpha_{\varepsilon}$ because $\sigma \cdot \sigma=1+\varepsilon$. Taking determinants in

$$
\tau_{12} \tau_{13} \tau_{23}=\tau_{1} \tau_{2} \tau_{3} \tau_{4}
$$

leads to:

$$
\alpha_{\varepsilon}^{3}=\alpha_{\sigma}^{8}
$$


$\tau_{i}=1,2,3,4$ is proportional to the $2 \times 2$ unit matrix. Finally we consider $\varepsilon \varepsilon \sigma \sigma$. Now $\tau_{12}$ has eigenvalue $1, \tau_{13}$ has eigenvalue $\alpha_{\sigma}$ and $\tau_{23}$ has eigenvalue $\alpha_{\sigma}$ and $\tau_{1} \tau_{2} \tau_{3} \tau_{4}$ yield $\alpha_{\sigma}^{2} \alpha_{\sigma}^{2}$. Hence

$$
\alpha_{\epsilon}^{2}=1
$$

This implies (5.27) and leads to $\alpha_{\varepsilon}=\alpha_{\sigma}^{8}$. Then the possible values of $h_{\varepsilon}, h_{\sigma}$ are:

$$
2 h_{\varepsilon}=\text { integer } \quad 8 h_{\sigma}=h_{\epsilon}+\text { integer } .
$$

This is obviously satisfied by the Ising model with $h_{6}=1 / 2, h_{\sigma}=1 / 16$ and for example by the SU(2) WZW theory at level two. The primary fields are $1, \phi_{1 / 2}, \phi_{1}$ with dimensions $h_{0}=0, h_{1 / 2}=3 / 16, h_{1}=1 / 2$ which clearly satisfies (5.30). In this case the solutions to (5.30) are parameterized by two integers. In general the dimensions $h_{i}, \ldots, h_{n}$ of the fields will be determined only mod. 1. It is not known one can obtain extra conditions which put upper bounds on the integers solving equations like (5.27)(5.29). Using such relations for a general RCFT C. Vafa showed that the dimensions $h_{i}$ are always rational numbers in a RCFT. Using $(S T)^{3} \equiv C$ it is also easy to show that $c$ is a rational number. Taking determinants:

$$
\operatorname{det}(S T)^{6}=1 \Rightarrow \operatorname{det} T^{6}= \pm 1
$$

Since the eigenvalues of $T$ are $\exp 2 \pi i\left(H_{i}-c / 24\right)$ we obtain:

$$
e^{2 \pi i c / 24}= \pm \prod_{i=1}^{n} \alpha_{i}^{6} .
$$

Hence $c / 4=6 \sum h_{i} \bmod 1$, proving the rationality of $c$. As a final example of the use of these general algebraic identities we show that a theory with only one field different from the identity: $\phi$ can only have fusion rules of form $\phi \times \phi=1$ or $\phi \times \phi=1+\phi$. If we try to impose $\phi \times \phi=1 \times n \phi n \geq 2$, we run into inconsistencies. The argument is very simple. The field $\phi$ is self-conjugate, $C=1$ and $(S T)^{3}=1$. The eigenvalues $\lambda$ of $N_{\phi}$ satisfy the quadratic equation

$$
\lambda^{2}=1+n \lambda
$$

also we can parametrize $S$ and $T$ as

$$
S=\left(\begin{array}{cc}
\cos \theta & \sin \theta \\
\sin \theta & -\cos \theta
\end{array}\right) \quad T=e^{-i \pi c / 12}\left(\begin{array}{cc}
1 & 0 \\
0 & \alpha_{\phi}
\end{array}\right) \alpha_{\phi}=e^{2 \pi i h_{\phi}}
$$

The condition that the fusion rules are diagonalized by $S$ implies $\tan \theta=\lambda$; and $(S T)^{3}=1$ leads to

$$
\cos 2 \pi h_{\phi}=-\frac{1}{2} n \lambda, \quad 12 h-c=2(\bmod 8) .
$$

Next consider blocks of the form $\phi \phi \phi \phi$. There are $n^{2}+1$ such blocks. $\tau_{i j}$ has $n^{2}$ eigenvalues equal to $\alpha_{\phi}$ and the remaining eigenvalue $\tau$ equal 1 . Taking determinants in $\tau_{12} \tau_{12} \tau_{23}=\tau_{1} \tau_{2} \tau_{3} \tau_{4}$ we arrive at

$$
\left(\alpha_{\phi}\right)^{n^{2}+4}=1
$$

The equations:

$$
\left(\alpha_{\phi}\right)^{n^{2}+4}=1 \quad \lambda^{2}=1+n \lambda, \quad \alpha_{\phi}+\alpha_{\phi}^{-1}=-n \lambda
$$

are compatible only for $n=0$ or 1 . For $n=0$ one example is provided the level $1 \mathrm{SU}(2) \mathrm{WZW}$ model. With $n=1$ the examples are the the level 1 $G_{2}$ and $F_{2}$ WZW models. It is clear from (5.37) and the reality of both $h_{\phi}, \lambda$ that $n \leq 2$. For $n=0,1$ we already know of some solutions, so the inconsistency only appears for $n=2$ because $\lambda=1 \pm \sqrt{2}$ and $\alpha_{\phi}^{8}=1$ do not solve the last equation in (5.37).

For a small number of generators it is possible to determine all possible fusion rules. However, once the number of fields different from the identity is larger or equal to 3 , the brute force classification seems hopeless and new ideas should come into play if we want to classify the possible fusion rules appearing in RCFT. In the next two sections we will argue that the classification of RCFT is intimately connected to the problem of classified Quasi-Triangular Yang-Baxter algebras (Quantum Groups for short) with some rationality constraint on their representation ring. If this conjecture is true, the fusion rules for RCFT are classified by Clebsch-Gordan decompositions of restricted tensor products of representations of Quantum groups.

We would like to conclude this section with the definition of dimension for a conformal family introduced by Dijkgraaf and Verlinde ${ }^{53}$. Dividing the character $\chi_{i}(\tau)$ of some family by the character of the identity and taking the $\tau \rightarrow 0$ limit gives a regularized version of the dimension of $X_{i}$. For finite dimensional spaces, $\chi_{i}(0)=$ dim $h_{i}$, so this definition appears reasonable:

$$
d_{i} \equiv \lim _{\tau \rightarrow 0} \frac{\chi_{i}(\tau)}{\chi_{0}(\tau)}
$$


To compute $d_{i}$ we use the modular transformation $S$ and the fact that in unitary theories $h_{i} \geq 0$, and $h_{i}=0$ for the identity operator. Then:

$$
d_{i}=\lim _{\tau \rightarrow 0} \frac{\sum_{j} S_{i}^{j} \chi_{j}(-1 / \tau)}{\sum_{k} S_{0}^{k} \chi_{k}(-1 / \tau)}=\frac{S_{i}^{0}}{S_{0}^{0}}
$$

We will show later how to compute (5.39) for WZW models and how they relate to characters of quantum groups.

\section{Duality and Quantum Groups}

\subsection{Motivation}

In the first few sections we have reviewed some of the basic properties of CFT. The duality or bootstrap hypothesis provided a set of equations for the OPE coefficients in CFT. Furthermore modular invariance at genus one gives constraints on the possible operators algebra. It is still quite hopeless to try a direct classification of all CFT by solving the bootstrap together with the modular invariance constraints. A resolution of this problem would determine the universality classes of two-dimensional critical phenomena and it would determine completely the classical vacuum manifolds for string and superstring theories. The problem can be drastically simplified if we include a rationality assumption. We want to first classify rational conformal field theories RCFT i.e. those CFT with a finite number of primary felds with respect to some chiral algebra. We have seen that the duality properties of a RCFT are summarized by the hexagon and pentagon identities at genus zero and at genus one we have the relations $S^{2}=(S T)^{3}=c$. If these conditions are supplemented with the requirement of modular invariance for the one-point functions at genus one, Moore an Seiberg showed that the theory is guaranteed to be modular invariant on a Riemann surface of arbitrary genus. Hence the duality properties are coded in a collection of polynomial equations ${ }^{16}$. Understanding of the space of solutions of the polynomial equations is an important step in the classification of RCFT. In this subsection we analyze the duality properties of a RCFT and argue that their solutions are given by the representation theory of Quantum Groups when the deformation parameters are roots of unity. In Ref. 18 it was shown that the duality properties of these RCFT admitting a classical limit can be described in terms of the representation ories with a classical limit fall in sequences of theories with a parameter

playing the role of Planck's constant. For instance in WZW theories we have a group $G$ and a level $k$ for the Kac-Moody algebra. The classical limit is obtained as $k \rightarrow \infty$. For many of the known sequences the duality properties of their elements can be obtained by quantizing à la Drinf'eldJimbo the group appearing in the classical limit. For theories which do not admit a classical limit (if they exist) we may have a situation similar to the classification of exceptional hie group or sporadic finite simple groups.

Geometrically the conformal blocks can be described as sections of a (projectively) flat holomorphic vector bundle over the moduli space $M_{8, n}$ of genus $g$ surfaces with $n$ distinguished points (we may also want to include local parameters about the distinguished points to resolve the orbifold singularities in $\left.\mathcal{M}_{g, n}\right)$. At genus zero we have $S^{2}-\left\{P_{1}, \ldots, P_{n}\right\}$ and after three points are fixed by $\mathrm{SL}_{2}(\mathrm{C})$ invariance the Teichmuller space can be described in terms of $n-3$ copies of $S^{2}$ removing the "diagonal" subsets where two or more points coincide. (The geometrical aspects and their relevance to CFT and string theory has been emphasized by Friedan and Shenker in their attempts to formulate CFT in some Universal Moduli space). The group $\operatorname{Diff}^{+}\left(S^{+}-\left\{P_{1}, \ldots P_{n}\right\}\right)$ (including maps permuting the points but preserving the orientation) acts on the space of blocks as a consequence of duality. If Diff $\operatorname{Di}^{+}\left(S^{2}-\left\{P_{1}, \ldots, P_{n}\right\}\right)$ is the subset of diffeomorphisms connected with the identity, the duality properties are related to the representations of the quotient group Diff + Diff $f_{0}^{+}$furnished by the conformal blocks. Since the bundle of conformal blocks is flat, we can use a flat connection (naturally given by the energy-momentum tensor) to identify all the fibers with one of them and study the action of the quotient group on this fiber. Taking into account that the duality properties are the same for primary an secondary fields, the duality properties only involve finite dimensional vector spaces. Formally if $\lambda$ is the Hilbert space of the theory and $\sigma$ the chiral algebra we are dealing with $K / \sigma$. In the decomposition $K=\oplus_{i, i} K_{i} \times K_{i}$ only the highest weight subspaces $V_{i} \subset K_{i}$ survive. The subspace $V_{i}$ is spanned by all elements in $K_{i}$ with the same $L_{0}$ eigenvalue as the highest weight vector. If the chiral algebra has a well-defined zero mode subalgebra the spaces $V_{i}$ are representations of the zero mode algebra.

If $\phi_{1}, \ldots \phi_{N}$ are the primary fields of the theory, a basis of the space of blocks with the $\phi_{i}$ on the external legs is obtained by describing a way of decomposing $V_{i} \times \ldots \times V_{N}$ according to the fusion rules. This generates a collection of skeleton graphs with labels on the external and internal lines. This decomposition is reminiscent of group theory, albeit not classical 
group theory. Whatever the algebra structure underlying the decomposition of $V_{i} \times \ldots \times V_{N}$ is, it has to contain the duality properties of the CFT if its representation theory is to stand a chance of solving the polynomial equations. To see that this requirement forces us in general to deviate from ordinary group theory, we can think of the three-point block in Fig. 24 as an intertwinner in the algebra $A$ which embodies the extension by the duality operations of the zero mode subalgebra of the chiral algebra. If we exchange (braid) the $i, j$ legs in Fig. 24 in the classical case this will only amount to a sign counting whether the representation $k$ appears symmetrically or antisymmetrically in the tensor product $V_{i} \otimes V_{j}$. In CFT this exchange produces this sign together with a phase depending on the conformal weights of the $i, j, k$ fields. If $\sigma_{i j}$ represents the braiding of $i$ and $j$ (the $\Omega$ operation in Refs. 16 and 18) and $F_{k}^{i j}$ is the block in Fig. 24, then:

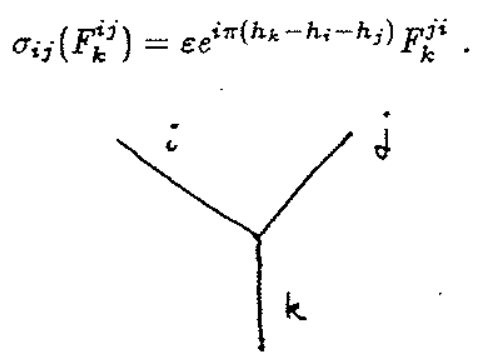

Fig. 24. Three point block as an intertwinner.

$\varepsilon$ is a sign with the same interpretation as in the classical case. The two basic moves $F, B$ on the space of blocks appear in Fig. 4 and they are related by the moves in Fig. 9 , or in equations:

$$
B_{p p^{\prime}}\left[\begin{array}{cc}
j & k \\
i & l
\end{array}\right]=\varepsilon_{k l}^{p} \varepsilon_{p^{\prime} k}^{i} e^{i \pi\left(h_{p}+h_{p^{\prime}}-h_{i}-h_{l}\right)} F_{p p^{\prime}}\left[\begin{array}{ll}
j & l \\
i & k
\end{array}\right]
$$

We can interpret the $B, F$ operations in (quantum) group theoretic language. Although the precise definitions of quantum groups and quasitriangular algebras will appear in the next subsection, we want to motivate these definitions from the point of view of the duality properties of a RCFT. In ordinary group theory the intertwinners can be used to define the composition of angular momentum. If $K_{k}^{i j}: V_{i} \otimes V_{j} \rightarrow V_{k}$ is an intertwinner and the algebra $A$ acts on $V_{k}$ via the matrices $\rho^{k}(a), a \in A$ we can define the action of $A$ in the tensor product $V_{i} \otimes V_{j}$ via the diagram:

$$
\begin{array}{cc}
V_{i} \otimes V_{j} & \stackrel{K_{k}^{i j}}{\longrightarrow} V_{k} \\
\left.\rho_{i} \otimes \rho_{j}(\Delta(a))\right\rfloor & \\
V_{i} \otimes V_{j} \stackrel{K_{k}^{i j}}{\longrightarrow} V_{k}
\end{array}
$$

Technically this is the same as defining a co-multiplication operations in the algebra $A: \Delta: A \rightarrow A \otimes A$, and $\rho^{i} \otimes \rho^{j}(\Delta(a)) \equiv \Delta^{i j}(a)$. If $A$ and the tensor product of representations are associative, then the co-multiplication $\Delta$ is also associative. As in (6.1) the intertwinner will have non-trivial monodromy, and this is also reflected in the co-multiplication. Once $\Delta$ is defined, we can construct the action of $A$ on arbitrary tensor products of representations by iterating the co-multiplication. Exploring further our identification of conformal blocks with the decomposition of the tensor product $V_{j_{1}} \otimes \ldots \otimes_{i_{N}}$ :

$$
V_{i_{1}} \otimes \ldots \otimes V_{i_{N}}=\bigoplus_{j} F_{j}^{i_{1} \ldots i_{N}} \otimes V_{j}
$$

the space $F_{j}^{i_{1} \ldots i_{N}}$ is generated by the blocks $f_{j}^{i_{1} \ldots i_{N}}\left(p_{1}, \ldots, p_{N-2}\right)$ with one spectator field in the representation $j$ and $p_{i}, \ldots, p_{N-2}$ are possible intermediate states according to the fusion rules in the basis for the decomposition (6.4) shown in Fig. 25.

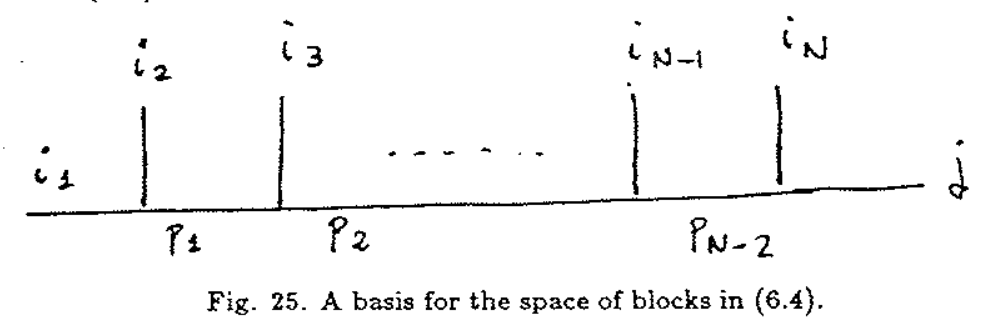

The multiplicity of the representation $V_{j}$ in (6.4) is given by the dimension of the space $f_{j}^{i_{1} \ldots i_{N}}$. There are some properties of (6.4) which follow from the general structure of RCFT. In the decomposition (6.4) we have assumed the representation on the left-hand side to be not only reducible but also fully reducible. This is not the case for general algebras but it holds in 
CFT. The space of all conformal blocks can be divided into subsets, each providing an irrep. of the braid group acting on the external legs. From general arguments in representation theory the blocks $\xi_{j}^{i_{1} \ldots i_{N}}$ provide representations of the centralizer of the algebra $A$ acting on the tensor product $V_{i_{1}} \otimes \ldots \otimes V_{i_{N}}$. By defunition of $A, B$ are two algebras acting on the same space $V, B$ is the centralizer of the action of $A$ on $V$ is the set of operators $b: V \rightarrow V$ commuting with the action of $A$. If $A, B$ are both semisimple and finite dimensional then if $B$ is the centralizer of $A$ in $V, A$ is the centralizer of $B$. The importance of the centralizer in representation theory originates from Schur's lemma. Consider for example the Lie algebra gl( $N)$, and let $V$ be the defining $N$-dimensional representation. In the decomposition of $V \otimes \ldots \otimes V$ into irreducible representations we first look for the algebra which centralizes the action of $\operatorname{gl}(N)$ on it. If the centralizer algebra $B$ is semisimple, the irreducible representations appearing in $V \otimes \ldots \otimes V$ are labeled by the irreducible representations of $B$. The centralizer algebra of the action of $g l(N)$ on $V \otimes \ldots \otimes V=V^{\otimes n}$ is generated by the permutations exchanging the factors with the constraint that the $N+1$-row antisymmetrizer vanishes; i.e. this is equivalent to saying that only tableaux with at most $N$ rows appear in the irreducible representations of $g l(N)$.

In CFT we start with (6.4) and for simplicity assume for the time being $V_{i_{1}}=V_{i_{3}}=\ldots=V_{i_{N}}=V_{\phi}$. The argument extends easily to the general case. These particular blocks are represented in Fig. 26. Since the braiding properties in CFT are independent of descendants, we expecr some representation of the braid group to be the centralizer of the algebra $A$. If we knew the algebra $A$ explicitly we could in principle find its centralizer $B$, whose representations would completely characterize the decomposition of the tensor product $V_{\phi}^{\otimes n}$. The complete reducibility of the braid representations provided by the conformal blocks implies that the centralizer algebra must be semisimple. Later we will see that in the more interesting case (quanturn groups whose deformation parameters are roots of unity) some restrictions have to be imposed on their representation theory in order to obtain effectively a semisimple centralizer. (This is equivalent to dividing the centralizer by some Abelian ideals and working with the semisimple quotient). Without going into these complications at the moment let us assume the centralizer to be semisimple. From the duality principle in CFT the braid group $B_{N}$ acts on the blocks on the right-hand side of (6.4). a representation of the braid group. Reading (6.4) backwards and using the

braid representation on the blocks we can construct a matrix $R^{\phi \phi}$ acting on contiguous spaces in the tensor product. This matrix commutes with the co-multiplication operation i.e. it commutes with $\Delta(a), a \in A$ by the definition of centralizer,

$$
R^{\phi \phi} \Delta^{\phi \phi}=\Delta^{\phi \phi} R^{\phi \phi}
$$

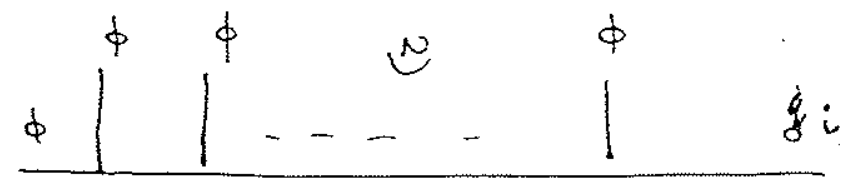

Fig. 26.

If the representations on the external legs are different, the analogue of $(6.5)$ is

$$
R^{i j} \Delta^{i j}=\Delta^{j i} R^{i j}
$$

where:

$$
R^{i j}: V^{i} \otimes V^{k} \rightarrow V^{j} \otimes V^{i}
$$

If $P^{i j}: V_{i} \otimes V_{j} \rightarrow V_{j} \otimes V_{i}$ is the permutation map $P^{i j}(x \otimes y)=y \otimes x$ we can define

$$
\begin{aligned}
& R^{i j}=P^{j i} R^{i j}: V_{i} \otimes V_{j} \rightarrow V_{j} \otimes V_{i} \\
& p^{i j} p^{j i}=1
\end{aligned}
$$

and (6.6) can be interpreted at the level of the algebra $A$ by saying that once $A$ is endowed by a co-multiplication $\Delta$, we can always construct a second one $\Delta^{\prime}=\sigma \circ \Delta$, where $\sigma=A \otimes A \rightarrow A \otimes A$ is the permutation map. Equation (6.6) says that $\Delta^{\prime}$ and $\Delta$ are conjugate within $A \otimes A$; the exist a matrix $R: A \otimes A \rightarrow A \otimes A$ so that

$$
\Delta^{\prime}(a)=\sigma \circ \Delta(a)=R \Delta(a) R^{-1} .
$$

This can be seen using

$$
P^{i j} \Delta^{i j i} P^{j i}=\Delta^{i j} \text {. }
$$

Two extra conditions on the co-multiplication are obtained from the fact that in CFT fusing and braiding are compatible. This is illustrated in 
Fig. 27. When written in equations the equalities in Fig. 27 imply two of the defining relation for a Quasi-Triangular Yang-Baxter algebra which together with (6.9) provide all but one axiom for this algebraic structure. The missing axiom can be explained in terms of the relation between the braiding and fusing properties of a set of fields and those of their conjugates. A simple consequence of these axions is the Yang-Baxter equation for the matrix $R$ and the hexagon and pentagon identities. Furthermore the modular transformation matrix $S$ with all the properties (5.18) can be obtained from the properties of the co-multiplication for these algebras ${ }^{25}$.

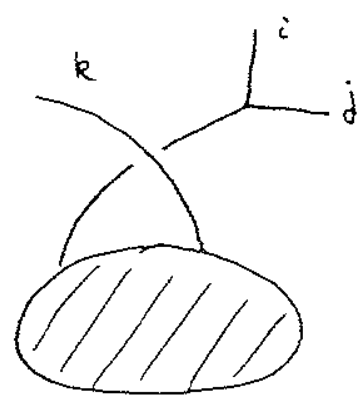

$=$
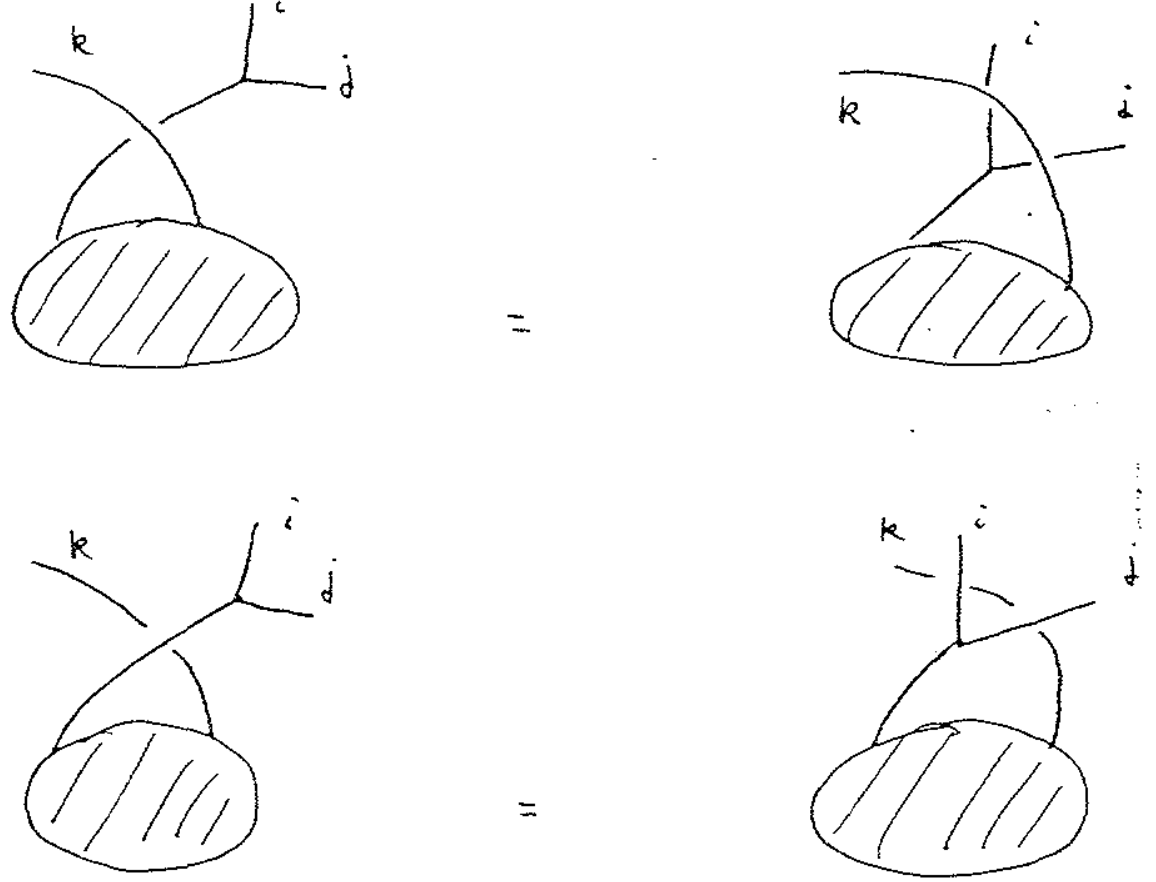

Fig. 27. Compatibility between braiding and fusion.

Although in general we do not yet know whether it is possible to associate a quantum group to every RCFT in some case it is not difficult to derive the quantum groups whose representation theory reproduce their duality properties. Ideally one would like to find an explicit construction of the quantum algebra $\beta$ in terms of the generators of the chiral algebra $\sigma$. There are several ways of constructing quantum group actions on the space of blocks for $\mathrm{RCFT}^{22,24}$, however it is not known at present how to relate them to $\sigma$. The previous arguments are helpful in understanding why such a construction may not be so simple. The duality properties encoded by the quantum group correspond to rather nonlocal operations in terms of the actual blocks (they involve braiding, analytic continuation...). Hence the quantum group generators should be nonlocal functional of the generators of $\sigma$. We and it more appealing (and easiex) to find the quantum group as the centralizer of the braid group action on the blocks and then use its representation theory to construct explicitly the matrices $B, F, S, T$ satisfyin the polynomial equations. We will work out some examples in details later. This subsection was only intended to motivate the defining relations of quantum groups from the point of view of RCFT. It is also quite clear that the matrices $B, F, S, T$ convey all the topological information contained in a RCFT. This will be exploited and explained in Sec. 9 where we present in some details the connection of CFT with three-dimensional topology and the Chern-Simons approach to both subjects. After these preliminaries we study the general structure of quantum group and their representation theory when the deformation parameters are roots of unity.

\subsection{Quartum groups and their representations for $q$ a root of unity}

Given an associative algebra $A$ with unity, we say that $A$ is a Hopf algebra (general references on Quantum Groups are Refs. 26,27,28,29 and 31) if we can define three operations $\Delta, \gamma, \varepsilon$ on $A ; \Delta: A \rightarrow A \otimes A$ is the co-multiplication, $\gamma: A \rightarrow A$ is the antipodal map and $\varepsilon: A \rightarrow \mathbb{C}$ is the counit. $\mathbb{C}$ is the field over which $A$ is an algebra. The operations $\Delta, \varepsilon$ are algebra homomorphisms. $\Delta(a b)=\Delta(a) \Delta(b), \varepsilon(a b)=\varepsilon(a) \varepsilon(b)$ whereas $\gamma$ is an anti-homomorphism, $\gamma(a b)=\gamma(b) \gamma(a)$. These three operations must satisfy the following axioms:

$$
\begin{aligned}
& a, b \in A,(i d \otimes \Delta) \Delta(a)=(\Delta \otimes i d) \Delta(a) \\
& m(i d \otimes \gamma) \Delta(a)=m(\gamma \otimes i d) \Delta(a)=\varepsilon(a) 1 \\
&(\varepsilon \otimes i d) \Delta(a)=(i d \otimes \varepsilon) \Delta(c)=a
\end{aligned}
$$

where $m$ is the multiplication in the algebra; $m: A \otimes A \rightarrow A, M(a \otimes b)=a \cdot b$. In words, the first condition is the associativity of the co-multiplication; the second is the definition of the antipode and the third defines the co-unit. If $\sigma=A \otimes A \rightarrow A \otimes A$ is the permutation map $\sigma(a \otimes b)=b \otimes a$, it is easy to check that $\Delta^{\prime}=\sigma \circ \Delta$ is another co-multiplication in $A$ with antipode 
$\gamma^{\prime}=\gamma^{-1}$. A Hopf algebra is a quasi-triangular Yang-Baxter algebra if the co-multiplications $\Delta, \Delta^{\prime}$ are related by conjugation:

$$
\sigma \cdot \Delta(a)=R \Delta(a) R^{-1} \quad R \in A \times A
$$

and the following conditions are satisfied

$$
\begin{aligned}
& (i d \otimes \Delta)(R)=R_{13} R_{12} \\
& (\Delta \otimes i d)(R)=R_{13} R_{23} \\
& (\gamma \otimes i d)(R)=R^{-1} .
\end{aligned}
$$

Since $(i d \otimes \Delta)(R) \in A \otimes A \otimes A, R_{13}$ acts as the identity in the second factor and as $R$ in the first and third, and similarly for $R_{12}, R_{23}$. The motivation for these axioms came originally from the theory of integrable models (see Ref. 26 and references therein). In CFT (6.12) comes from 26 the definition of the centralizer and the first two conditions in (6.13) are the precise statement of the compatibility between fusing and braiding represented graphically in Fig. 27. An immediate consequence of these axioms is the Yang-Baxter equation for $\mathcal{R}$. Write

$$
R=\sum_{i} a_{i} \otimes b_{i}
$$

Then

$$
R_{13} R_{23}=\sum_{i, j} a_{i} \otimes a_{j} \otimes b_{i} b_{j}
$$

and:

$$
\begin{aligned}
{[\sigma \circ \Delta \otimes i d] R } & =\sum_{i} \Delta^{\prime}\left(a_{j}\right) \otimes b_{i}=\sum_{i} R_{12} \Delta\left(a_{i}\right) R_{12}^{-1} \otimes b_{i} \\
& =R_{12} \sum_{i} \Delta\left(a_{i}\right) \otimes b_{i} R_{12}^{-1}=R_{12}(\Delta \otimes i d(R)) R_{12}^{-1} \\
& =R_{12} R_{13} R_{23} R_{12}^{-1} .
\end{aligned}
$$

yielding

$$
R_{12} R_{13} R_{23} R_{12}^{-1}=R_{23} R_{13}
$$

or:

$$
R_{12} R_{13} R_{23}=R_{23} R_{13} R_{12} .
$$

The matrix $R$ is known as the universal $R$-matrix for the algebra $A$.

As an example consider any Cartan matrix $\left(a_{i j}\right)$ for an ordinary simple Lie group or untwisted affine Kac-Moody algebra. With it we can associate a deformation of the classical algebra ${ }^{26}$. Working in a Chevalley basis, to every root we associate three generators $X_{i}^{+}, X_{i}^{-}, H_{i}$ satisfying the relations:

$$
\begin{aligned}
& {\left[H_{i}, H_{j}\right]=0} \\
& {\left[H_{j}, X_{i}^{ \pm}\right]= \pm\left\langle\alpha_{i}, \alpha_{j}\right\rangle X_{i}^{ \pm} \quad\langle a, b\rangle \equiv 2 a \cdot b / b^{2}} \\
& {\left[X_{i}^{+}, X_{j}^{-}\right]=\delta_{i j} \frac{q^{H_{i} / 2}-q^{-H_{i} / 2}}{q^{1 / 2}-q^{-1 / 2}}} \\
& {\left[X_{i}^{ \pm}, X_{j}^{ \pm}\right]=0 \quad \text { if } a_{i j}=0} \\
& \sum_{\nu=0}^{1-a_{i j}}(-1)^{\nu}\left[\begin{array}{c}
1-a_{i j} \\
\nu
\end{array}\right]_{q_{i}}\left(X_{i}^{ \pm}\right)^{1-a_{i j}-\nu} X_{j}^{ \pm}\left(X_{i}^{ \pm}\right)^{\nu} q_{i}^{-\nu\left(1-\alpha_{i j}-\nu\right) / 2}=0 \\
& i \neq j \quad q_{i}=q^{\alpha_{i}^{2} / 2}
\end{aligned}
$$

where we use the definitions for $q$-numbers:

$$
\begin{aligned}
& {[X]_{q}=\frac{q^{X / 2}-q^{-X / 2}}{q^{1 / 2}-q^{-1 / 2}}} \\
& {\left[\begin{array}{c}
n \\
m
\end{array}\right]=\frac{[n] !}{[m] ![n-m] !},[n] !=[n][n-1] \ldots[2][1]}
\end{aligned}
$$

the operators $\Delta, \gamma, \varepsilon$ are defined by:

$$
\begin{aligned}
& \Delta\left(\mathscr{H}_{i}\right)=\mathscr{K}_{i} \otimes 1+1 \otimes \mathscr{H}_{i} \\
& \Delta\left(X_{i}^{ \pm}\right)=X_{i}^{ \pm} \otimes q^{X_{i} / 4}+q^{-\mathscr{H}_{i} / 4} \otimes X_{i}^{ \pm} \\
& \varepsilon(1) \quad=1 \quad \varepsilon\left(X_{i}^{ \pm}\right)=\varepsilon\left(\mathscr{K}_{i}\right)=0 \\
& \gamma\left(X_{i}^{ \pm}\right)=-q^{\rho / 2} X_{i}^{ \pm} q^{-\rho / 2} \\
& \rho \quad=\frac{1}{2} \sum_{\alpha>0} \not{K}_{\alpha} .
\end{aligned}
$$


It is an easy exercise to check that (6.17) satisfies the axioms of a Hopf algebra. Moreover (6.15) also has the structure of a quasi-triangular YangBaxter algebra (quantum group for short). ${ }^{26-29}$ To keep the discussion as simple as possible consider the $\mathrm{SU}(2)$ case. Now we do not have the last relation in (6.15) (the q-analog of the Serre relations) and the defining properties are:

$$
\begin{aligned}
& {\left[X^{+}, X^{-}\right]=[K]} \\
& {\left[K, X^{ \pm}\right]= \pm 2 X^{ \pm}} \\
& \varepsilon\left(X^{ \pm}\right)=\varepsilon(K)=0, \varepsilon(1)=1 \\
& \gamma\left(X^{ \pm}\right)=-q^{ \pm 1 / 2} X^{ \pm}, \gamma(K)=-H
\end{aligned}
$$

with the universal $R$ matrix given by ${ }^{27,29}$ :

$$
R=q^{X \otimes K / 4} \sum_{n \geq 0} \frac{\left(1-q^{-1}\right)^{n}}{[n] !} q^{-n(n-1) / 4} q^{n K / 4}\left(X^{+}\right)^{n} \otimes q^{-n H / 4}\left(X^{-}\right)^{n}
$$

and one can check that it satisfies (6.12) and (6.13).

As long as $q$ is not a root of unity, the representation theory of quantum groups runs parallel with the classical theory. The representations are labelled by the same highest weight vectors and most of the standard expressions valid for classical algebras have $q$-analogs which often amount to replacing ordinary numbers by $q$-numbers $[X]$. From the point of view of representation theory the co-multiplication is analogous to the rule for addition of angular momentum. Geometrically for $q$ generic we can understand the representation theory of $\mathrm{SU}(2, q)$ as a deformation of the Borel-Weil construction. The fundamental representation of $\mathrm{SU}(2)$ has two states: spin up and spin down. We can represent these two states by two variables $u, v$ respectively. If we consider the space of polynomials on these two variable the irreducible representation of $\mathrm{SU}(2)$ with spin $j$ is given by the homogeneous polynomials of degree $2 j$. The generators of SU(2) become the differential operators:

$$
X^{+}=u \frac{\partial}{\partial v} \quad X^{-}=v \frac{\partial}{\partial u} \quad \nVdash=u \frac{\partial}{\partial u}-v \frac{\partial}{\partial v} .
$$

A basis for the spin $j$ representation is given by the monomials:

$$
|j, m\rangle=\frac{u^{j+m} v^{j-m}}{\sqrt{(j+m) !(j-m) !}} \quad m=-j,-j+1, \ldots, j
$$

orthonormal with respect to the metric:

$$
\langle f \mid g\rangle=\left.f\left(\frac{d}{d u}, \frac{d}{d v}\right) g(u, v)\right|_{u=v=0}
$$

Geometrically we can describe the sphere $S^{2}$ as $\mathrm{CP}^{1}$ in terms of two complex homogeneous coordinates $u, v$. In this representation the vectors (6.21) are holomorphic sections of the space of $-j$-differentials on the sphere (i.e. $f(z, \bar{z}) d z^{-j}$ is invariant under holomorphic coordinate changes). According to the Rieman-Roch theorem the space of holomorphic $-j$-differential $H^{\circ}\left(S^{2}, K^{-j}\right)$ has dimension $2 j+1$, and in homogeneous coordinate (6.21) provides a basis of $\mathcal{H}^{0}\left(S^{2}, K^{-j}\right)$. The $q$-deformation of this construction is obtained by using the same polynomial ring in $u, v \mathrm{C}[u, v]$ but now the generators $X^{ \pm}$are represented by $q$-derivatives. The $q$-derivative of a function $f(u)$ is defined as:

$$
D_{u} f(u)=\frac{f\left(q^{1 / 2} u\right)-f\left(q^{-1 / 2} u\right)}{\left(q^{1 / 2}-q^{-1 / 2}\right) u}
$$

same for $D_{v}$ :

$$
D_{v} f(v)=\frac{f\left(q^{1 / 2} v\right)-f\left(q^{-1 / 2} v\right)}{\left(q^{1 / 2}-q^{-1 / 2}\right) v} .
$$

To compute the commutation relations of these $q$-derivatives we only need to compute them on the basic monomials $u^{a} v^{b}$. It is then straightforward to show that the operators:

$$
X^{+}=u D_{v} \quad X^{-}=v D_{u} \quad K=u \frac{\partial}{\partial u}-v \frac{\partial}{\partial v}
$$

satisfy the defining relations of $\mathrm{SU}(2, q)(6.18)$. The $q$-deformed scalar product is:

$$
\langle f \mid g\rangle=\left.f\left(D_{u}, D_{v}\right) g(u, v)\right|_{u, v}=0
$$

and with respect to this scalar product an orthonormal basis for the basis $j$ representation is given by:

$$
|j, m\rangle=\frac{u^{j+m} v^{j-m}}{\sqrt{[j+m] ![j-m] !}}
$$

and it is easy to derive formulae like:

$$
\left(X^{ \pm}\right)^{a}|j, m\rangle=\sqrt{\frac{[j \mp m] ! j \pm m+a \mid !}{[j \mp m-a] ![j \pm m] !}}|j, m \pm a\rangle .
$$


Finally the analogue of the Casimir operator is:

$$
C=X^{-} X^{+}+\left[\frac{\not H+1}{2}\right]^{2} \text {. }
$$

To make contact with the remarks in Sec. 6.1 about the centralizer algebra, we first consider the example $\mathrm{SU}(N, q)$. After this example the srategy for an arbitrary group $G$ should be clear. As with SU(2), the fundamental representations of $\mathrm{SU}(N)$ and $\mathrm{SU}(N, q)$ coincide. Suppose we want to decompose $V_{N} \otimes \ldots \otimes V_{N}$ into irreducible components. Defining the action of $\Delta(a), R$ and $R$ tensor products according to:

$$
\begin{aligned}
& \Delta^{i j}(a)=\rho^{i} \otimes \rho^{j}(\Delta(a)): V_{i} \otimes V_{j} \rightarrow V_{j} \otimes V_{i} \\
& R^{i j}=\rho^{i} \otimes \rho^{j}(R) \\
& R^{i j}=P^{i j} R^{i j}: V_{i} \otimes V_{j} \rightarrow V_{j} \otimes V_{i} .
\end{aligned}
$$

Then from (6.12) we obtain

$$
R^{i j} \Delta^{i j}(a)=\Delta^{i j}(a) R^{i j}
$$

In our example $i, j$ are the fundamental representations and to simplify the notation we will remove in this case, the $i, j$ labels from $R^{i j}, \Delta^{i j}$. Then (6.30) becomes $R \Delta(a)=\Delta(a) R$ and the centralizer of $S U(N, q)$ in $V_{N}^{\otimes n}$ is generated by:

$$
\rho_{i}=1 \otimes \ldots \otimes R_{i, i+1} \otimes \ldots \otimes 1
$$

where the subindices in $R$ indicate the position of the spaces in the tensor product where $\rho_{i}$ acts nontrivially. From the definition of comultiplication plus the fact that the fundamental representations of $\mathrm{SU}(N, q)$ and $\mathrm{SU}(N)$ coincide we can compute $R$. if $e_{i j}$ is the $N \times N$ matrix unit whose single nonvanishing component is equal to 1 and it is located in position $(i, j)$, the answer is:

$$
R=\sum_{i \neq j} e_{i j} \otimes e_{j i}+q^{1 / 2} \sum_{i} e_{i i} \otimes e_{i i}+\left(q^{1 / 2}-q^{-1 / 2}\right) \sum_{i<j} e_{j j} \otimes e_{i i}
$$

and a straightfroward computation shows that

$$
R^{2}=\left(q^{1 / 2}-q^{-1 / 2}\right) R+1 .
$$

for convenience we define the generators $\rho_{i}$ by rescaling the $R$ matrix:

$$
\rho_{i} \equiv-q^{1 / 2} R \text {. }
$$

Then $\rho_{i}$ satisfies the defining relations for the Hecke algebra of type $A_{n}, \mathcal{K}(q)$ :

$$
\begin{array}{ll}
\rho_{i} \rho_{i \pm 1} \rho_{i} & =\rho_{i \pm 1} \rho_{i} \rho_{i \pm 1} \\
\rho_{i} \rho_{j} & =\rho_{j} \rho_{i} \quad|i-j| \geq 2 \\
\rho_{i}^{2} & =(1-q) \rho_{i}+q .
\end{array}
$$

The first equation in (6.35) follows from the Yang-Baxter equation. On $V_{i} \otimes V_{j} \otimes V_{k}$ the Yang-Baxter equation is:

$$
R^{i j} R^{i k} R^{j k}=R^{j k} R^{i k} R^{i j}
$$

and in terms of $(6.29 \mathrm{c})$ it becomes:

$$
\left(R^{j k} \otimes 1\right)\left(1 \otimes R^{i k}\right)\left(R^{i j} \otimes 1\right)=\left(1 \otimes R^{i j}\right)\left(R^{i k} \otimes 1\right)\left(1 \otimes R^{j k}\right) .
$$

The centralizer of $\operatorname{SU}(N, q)$ in $V_{N}^{\otimes n}$ satisfies the defining relations of the Hecke algebra $H_{n}(q)$ (plus one more relation; see below). We can proceed similarly for any representation $\mu$ and define a homorphism $\pi^{\mu}$ from the braid group $B_{n}$ into the centralizer algebra $C_{\mu}^{n}$ of $\mathrm{SU}(N, q)$ in $V_{\mu}^{\otimes n}$ :

$$
\pi^{\mu}\left(\sigma_{i}\right)=1 \otimes \ldots \otimes R_{i, i+1}^{\mu \mu} \otimes \ldots \otimes 1
$$

The extra relation alluded to above for $C_{N}^{n}\left(V_{\mu}=V_{N}\right)$ is the following: For $q$ generic, the irreps of $\mathrm{SU}(N, q)$ are labelled by the same Young tableaux as in the calssical case, the only difference is that the symmetrizer associated to a given tableaux is not constructed with the elementary transpositions $S_{i}$ of the symmetric group $S_{n}$ but rather with the generator $g_{i}$ of the Hecke algebra. The extra relations is equivalent to saying that the $(N+1)$-row antisymmetrizer vanishes. Only tableaux with at most $N$ rows appear. For example, in the $\mathrm{SU}(2, q)$ case the complete set of relations for $C_{2}^{\text {n }}$ are $(6.35)$ together with:

$$
1-\rho_{1}-\rho_{2}+\rho_{1} \rho_{2}+\rho_{2} \rho_{1}-\rho_{1} \rho_{2} \rho_{1}=0 .
$$


Equations (6.35) and (6.38) ar equivlent to the Temperley-Lieb-Jones algebra defined by $n-1$ projection operators $e_{i}$ :

$$
e_{i}=\frac{1-\rho_{i}}{1+q}
$$

satisfying the relations:

$$
\begin{aligned}
& e_{i} e_{i+1} e_{i}=\beta^{-1} e_{i} \quad \beta=2+q+q^{-1} \\
& e_{i}^{2} \quad=e_{i} \\
& e_{i} e_{j} \quad=e_{j} e_{i} \quad|i-j| \geq 2 \text {. }
\end{aligned}
$$

This algebra is known as $A_{\beta, n}$ and its properties hae been extensively studied by Jones. ${ }^{40,41}$ He has classified the subfactors when $q$ is a root of unity and analyzed the $C^{*}$ - representations. The centralizers $C_{N}^{n}$ of $\operatorname{SU}(N, q)$ in $V_{N}^{\otimes n}$ define a natural sequence of embeddings $\ldots \subset C_{N}^{n} \subset C_{N}^{n+1} \subset \ldots$ whose properties will be very useful in connecting RCFT and knot invariants (see Sec. 9.2). For $q$ generic $C_{N}^{n}$ is semisimple, hence any reducible finite dimensional representation of $\operatorname{SU}(N, q)$ is also fully reducible. From (6.30) we see that the centralizer of $C_{N}^{2}$ defines the co-multiplication operations in $\operatorname{SU}(N, q) . \operatorname{SU}(N, q)$ is the centralizer of $C_{N}^{n}$. This reciprocity relation will be useful later when we apply the remarks of Sec. 6.1 to compute the duality properties of some WZW model.

Before dealing with the case when $q$ is a root of unity we present one more property of the general theory ${ }^{28}$ that will be very useful in Sec. 9. For any representation $\mu$ we have the chain of embeddings . . . $C_{\mu}^{n} \subset C_{\mu}^{n+1} \subset \ldots$ and the formal direct limit $C_{\mu}^{\infty}$ describing the braiding operations in $V_{\mu}^{\otimes n}$ for any $n$. If $R^{\mu \mu}$ is $R^{i j}$ when $i=j=\mu$, and $\rho=\frac{1}{2} \sum_{\alpha>0} \mathscr{K}_{\alpha}(6.30)$ implies:

$$
R^{\mu \mu} q^{\rho} \otimes \rho^{\rho}=q^{\rho} \otimes q^{\rho} R^{\mu \mu}
$$

and we can use $\left(R^{\mu \mu}, q^{\rho}\right)$ to define a trace on the algebra $C_{\mu}^{\infty}$ with the properties:

$$
\begin{aligned}
& \operatorname{tr}(1)=1 \\
& \operatorname{tr} a b=\operatorname{tr} b a \\
& \operatorname{tr} \alpha \rho_{n}^{ \pm 1}=Z_{ \pm} \operatorname{tr} \alpha .
\end{aligned}
$$

The generators $\rho_{i}$ are defined in (6.37),

$$
Z_{ \pm}=\frac{q^{ \pm c(\mu)}}{\operatorname{tr}_{\mu} q^{\rho}}
$$

and $c(\mu)$ is the second Casimir for the classical group in the irrep. Labelled by the highest vector $\mu$. A trace with the property $(6.42 \mathrm{c})$ is known as a Markov trace, or a trace with the Markov property. This is crucial in connection with Knot theory. The trace is defined by

$$
\operatorname{tr} \alpha=\frac{1}{\operatorname{tr}_{\mu} q^{\varrho}}\left(q^{\varrho} \otimes \ldots q^{e} \alpha\right) .
$$

(For a proof see Ref. 28). Using the homomorphism (6.37) we get a Markov trace on the braid group.

This trace suggests the definition of a quantum dimension for a representation of a quantum group. If we remove the normalization factor from (6.44) and take the braid element to be the identity we can define the $q$-dimension of a representation of highest weight $\mu$ to be

$$
D_{q}(\mu)=\operatorname{tr} q^{\varrho}
$$

with tr the standard matrix trace. This definition will be shown to agree with the definition (5.38) and it plays a very important role in the representation theory of quantum groups when $q$ is a root of unity.

We finally come to analyze representations for quantum groups when the deformation parameter is a root of unity. Now the centralizer algebra and the quantum group are not semisimple and we want to find out under what conditions the representation theory can be restricted to a regular set of representations (modular representations of quantum groups appear in Ref. 31. A more physical treatments can be found in Ref. 32. Let us begin with $\mathrm{SU}(2, q)$. The basic problem when $q=e^{2 \pi i / p}$ is that $\left(X^{ \pm}\right)^{p}=0$ and this generates null vectors in some representations. Many of the representations appearing in the decomposition of tensor products of irreducible representations will be reducible but not fully reducible. Instead of using the basis (6.26), we construct a different basis for the representation with highest weight $j$ as

$$
\begin{aligned}
X^{+}|j\rangle & =0 \\
|m\rangle & =\frac{\left(X^{-}\right)^{j-m}}{[j-m] !}|j\rangle
\end{aligned}
$$


then

$$
\begin{aligned}
& X^{-}|j m\rangle=[j-m+1]|j, m-1\rangle \\
& X^{+}|j m\rangle=[j+m=1]|j, m+1\rangle \\
& \frac{\left(X^{ \pm}\right)^{a}}{|a| !}|j m\rangle=\frac{j \pm m+a \mid !}{[a] ! j+m] !}|j, m \pm a\rangle
\end{aligned}
$$

When $q^{p}=1,[p]=[2 p]=\ldots=\left[k_{p}\right]=0$, however the operators $\left(X^{ \pm}\right)^{p} /[p]$ ! are still well-defined as can be seen by taking $a=p$ in (6.47) for generic $q$ and then taking the limit $q \rightarrow e^{2 \pi i / p}$. It is also clear from the previous equations that $\left(X^{ \pm}\right)^{p}=0$ and moreover that this property is preserved by the co-multiplication as one should expect:

$$
\Delta\left(X^{ \pm}\right)^{N}=\sum_{j=0}^{N}\left[\begin{array}{c}
N \\
j
\end{array}\right] q^{-(N-j) H / 4}\left(X^{+}\right)^{j} \otimes q^{j H / 4}\left(X^{+}\right)^{N-j} .
$$

When $N=p$ the first and the last terms on the right-hand side contain $\left(X^{ \pm}\right)^{p}=0$ and they vanish, and any of the intermediated terms vanish because they are all proportional to $[p]$. Hence for large enough $j$ the representation will have null vectors. Following Ref. 32 we begin to see the source of the problem if we calculate the eigenvalues of the Casimir operator $(6.28)$ acting on highest weight vectors

$$
C|j\rangle=[j+1 / 2]^{2}|j\rangle
$$

for $q$ generic the eigenvalues of $C$ distinguish between all the different values of $j$. When $q=e^{2 \pi i / p}$, it is easy to see that $j, j+k p, p-1-j+k p$ for any integer $k$ have the same Casimir and if we compute the $q$-dimensions we find

$$
D_{q}(j)=D_{q}(j+k p)=-D_{q}(p-1-j+k p) .
$$

This suggests that if we try to decompose the tensor product $V_{1 / 2}^{\otimes n}$ for $n$ high enough into irreducible representations, odd things begin to happen. $V_{1 / 2}^{\otimes n}$ contains in its decomposition reducible but not fully reducible representations which pair up representations that would be distinct irreducible ones for $q$ generic adding up their $q$-dimensions to zero. For example if $q^{3}=1$ we can try to decompose $V_{1 / 2} \otimes V_{1 / 2} \otimes V_{1 / 2}$. For generic values of $q$ this tensor product decomposes into $V_{3 / 2} \oplus 2 V_{1 / 2}$. When $q^{3}=1$ the weight states of the spin $3 / 2$ and one of the spin $1 / 2$ representations mix into a reducible but not fully reducible representation due to the presence of null vectors. It is not difficult to check that the siate $|\alpha\rangle=X^{-}|1 / 2\rangle \otimes|1 / 2\rangle \otimes|1 / 2\rangle$ with $m=1 / 2$ is annihilated by $X^{+}$. Since this state is null, it is orthogonal to itself with respect to the metric (6.25) (notice that this scalar product is the standard one in terms of bras and kets with the prescription that in constructing bras from kets one should not complex conjugate $q$ ), and out of the other two states in $V_{1 / 2} \otimes V_{1 / 2} \otimes V_{1 / 2}$ only one of them is orthogonal to $|\alpha\rangle$. The other state $|\beta\rangle$ is not orthogonal to $|\alpha\rangle$ and therefore $X^{+}|\beta\rangle \propto|1 / 2\rangle \otimes|1 / 2\rangle \otimes|1 / 2\rangle$. The $q$ dimensions are $D_{q}(3 / 2)=[4]=$ $[3-4]=[-1]=-1$ and $D_{q}(1 / 2)=[2]=[3-2]=[1]=1$, where we have used $[p]=0,[x]=[p-x],[-x]=-[x]$. Thus $D_{q}(3 / 2)+D_{q}(1 / 2)=0$. Looking at other tensor product $V_{1 / 2}^{\otimes n}$ it is possible to prove that this situation is quite general. ${ }^{32}$ If we want to have a regular representation theory (so that tensor products of irreducible representations are fully reducible) we have to impose some conditions of the tensor product of representations. To find the integrable or regular representations when $p \geq 0$ we frest notice that $V_{1 / 2}$ has positive $q$-dimension. Since $[x]$ is a ratio of sine functions, we can solve the equation

$$
D_{q}(j)=[2 j+1]=0
$$

to find that the $q$-dimension vanishes whenever $j=\frac{p-1}{2}+k p$. As we keep on taking tensor products $V_{1 / 2} \otimes \ldots \otimes V_{1 / 2}$ we eventually obtain the representation with $j=\frac{p-1}{2}$ with vanishing $q$-dimension and other with positive $q$-dimensions. Tensoring another copy of $V_{1 / 2}$ begins the pairing of representations. We can restrict the representations of $\mathrm{SU}(2, q)$ to those with the smallest possible spin and positive $q$-dimension. This is achieved by requiring that in the tensor product of the fundamental representations we only keep those highest weight vectors annihilated by $X^{+}$and at the same time not in the image of $\left(X^{+}\right)^{p-1}$. This restricts the representations to those with spin smaller or equal to $(p-2) / 2$, defining $p=k+2$ we obtain the same restriction as in the $\mathrm{SU}(2)$ level $k \mathrm{WZW}$ model. In this way we find an alcove in the space of weights where the $q$-dimension is strictly positive with the lowest possible value of $j$. Using the scalar product (6.25)

$$
\left\langle j j\left|\frac{\left(X^{+}\right)^{n}\left(X^{-}\right)^{n}}{[n !][n !]}\right| j j\right\rangle=\left[\begin{array}{l}
j \\
n
\end{array}\right]
$$

it is easy to check the unitarity and positive definiteness of the representation. The representations with $j<(p-1) / 2$ are characterized by $: 1)$. 
They are highest weight representations. 2) The highest weight $|j\rangle$ cannot be written as $\left(X^{+}\right)^{p-1} \mid$ anything $\rangle$. The highest weights are in the space ${ }^{32}$

$$
|j\rangle \in \operatorname{Ker} X^{+} / \operatorname{Im}\left(X^{+}\right)^{p-1} \text {. }
$$

Next we can define a restricted tensor product. We have the collection of regular representations $\nu_{\text {reg }}=\left\{V_{j}, j \leq(p-2) / 2\right\}$. Given any two regular representations in $\nu_{\text {reg }}$ we construct the standard tensor product $V_{j_{2}} \otimes V_{j_{2}}$. If $j_{1}+j_{2} \leq(p-2) / 2$ the standard tensor product rule for $\mathrm{SU}(2)$ holds. As soon as the value of $j_{1}+j_{2}$ is surpassed, the highest weight vector with the largest value of $m_{1}+m_{2}$ will belong to $\operatorname{Im}\left(X^{+}\right)^{p-1}$, if $j=(p-1) / 2$ the $q$-dimension vanishes. For $j>(p-1) / 2$ the representation pairs with some other states into a reducible but not fully reducible representations. If we consider the set of highest weight vector $\left(V_{j i} \otimes V_{j_{2}}\right)^{+} \in V_{j_{i}} \otimes V_{j_{2}}$, we can restrict to those which are not in $\operatorname{Im}\left(X^{+}\right)^{p-1}$ and to the representation they generate. This will be our definition of the restricted tensor product

$$
\left(V_{j_{1}} \otimes V_{j_{3}}\right)^{\prime}
$$

where the prime stands for the operation of eliminating those representations whose highest weight $\in \operatorname{Im}\left(X^{+}\right)^{p-1}$, in other words eliminating those representations with vanishing $q$-dimension. Now the scalar product in $(6.25)$ is positive definite and the representation it provides is reducible and fully reducible. Furthermore, the coassociativity of the co-multiplication guarantees this tersor product to be associative

$$
\left.\left(\left(V_{j_{1}} \otimes V_{j_{2}}\right)^{\prime} \otimes V_{j_{3}}\right)^{\prime} \cong\left(V_{j_{3}} \otimes V_{j_{3}}\right)^{\prime}\right)^{\prime} .
$$

From the pairing argument described one easily gets the decomposition of (6.50) into irreducible regular representations

$$
\left(V_{j_{1}} \otimes V_{j_{2}}\right)^{\prime}=\bigoplus_{j=\left|j_{1}-j_{2}\right|}^{\min \left(j_{1}+j_{2}, k-j_{1}-j_{2}\right)} V_{j} \quad k=p-2 .
$$

Since the Bratelli decomposition of an algebra and that of its centralizer are the same, we see that the difinition (6.37) automatically leads to *representations of the Temperley-Lied-Jones algebra if $j_{1}=j_{2}=\ldots j_{n}=$ $1 / 2$ or the more complicated centralizer algebras for higher spins in the external legs.
Next we wish to consider a general group $G$ whose quantized commutation relations appear in (6.15). When $q$ is a root of unity we want to find the integrable representations and the conditions on the restricted tensor product. To find the regular alcove in the space of weight we use Weyl's character formula. For a representation with highest weight $\lambda$ we obtain for the $q$-dimension the expression

$$
D_{q}(\lambda)=\prod_{a>0} \frac{[\langle\lambda+e, \alpha\rangle]}{[\langle e, \alpha\rangle]} \quad \varrho=\frac{1}{2} \sum_{\alpha>0} \alpha .
$$

For $q^{p}=1$ we first determine when two representations will have equal or opposite $q$-dimension. The result $(6.52)$ is the same as the standard dimension formula except for the fact that numbers are replaced with $q$ numbers. For example in $\mathrm{SU}(N)(6.52)$ is the same as the rule of hooks to compute dimensions of irreducible representations. For a highest weight $\Lambda(4.66)$ its representation is given by the tableaux $\left[f_{1}, f_{2}, \ldots, f_{N \ldots 1}, 0\right]$. Using (4.62)-(4.66) we obtain for (6.52):

$$
D_{q}\left[f_{1}, \ldots, f_{N-1}, 0\right]=\prod_{1 \leq i<j \leq N} \frac{\left[f_{i}-f_{j}+j-i\right]}{[j-i]}
$$

and a few examples will convince the reader that this is the $q$-analogue of the staward formulae:

$$
\begin{aligned}
& D_{q}(\square)=[N] \\
& D_{q}(\mathrm{G})=\frac{[N][N-1]}{[2]} \\
& D_{q}(\theta k)=\frac{[N][N-1] \ldots[N-k+1]}{[k][k-1] \ldots[2] \cdot 1} \\
& \left.D_{q}(I]\right)=\frac{[N][N+1]}{[2]} \\
& D_{q}\left(\frac{k}{[-\cdots}\right]=\frac{[N][N+1] \ldots[N+k-1]}{[k][k-1] \ldots[2] \cdot 1}
\end{aligned}
$$


$D_{q}(N-1)=\frac{[N][N+1][N-1] \ldots[2]}{[N][N-2][N-3] \ldots[1]}=[N+1][N-1]$

etc. We now derive some properties of $(6.52)$. If $w_{i}$ is the Weyl reflection with respect to the simple root $\alpha_{i}$ then

$$
\prod_{a>0} \frac{\left[\left\langle w_{i}(\lambda)+w_{i}(\varrho), \alpha\right\rangle\right]}{[\langle\varrho, \alpha\rangle]}=-\prod_{a>0} \frac{[\langle\lambda+\varrho, \alpha\rangle]}{[\varrho, \alpha\rangle]}
$$

and for any element in the Weyl group:

$$
\prod_{\alpha>0} \frac{[\langle w(\lambda+\varrho), \alpha\rangle]}{[\langle\varrho, \alpha\rangle]}=\varepsilon(w) \prod_{a>0} \frac{[\langle\lambda+\varrho, \alpha\rangle]}{\langle\varrho, \alpha\rangle]}
$$

where $\varepsilon$ is the parity of $w$. Then we obtain

$$
D_{q}\left(w(\lambda+\varrho)-\varrho+P \sum_{i=1}^{r} n_{i} \alpha_{i}\right)=\varepsilon(w) D_{q}(\lambda), \quad q^{p}=1
$$

the $\alpha_{i}, i=1 \ldots, r$ are the simple roots of the classical algebra $G$. Every positive root can be written as $\alpha=\sum n_{i} \alpha_{i}, n_{i} \geq 0$, and $\sum n_{i} \equiv \operatorname{level}(\alpha)$. For the highest root $\theta$, the level plus one is the dual Coxeter number of the algebra $g=(\theta, \theta+2 \varrho) / \theta^{2}$. We will normalize the highest root to have length 2. For any weight $\lambda$, the largest value of $(\lambda+\varrho, \alpha), a>0$ is obtained for the highest root $\theta,\langle\varrho, \theta\rangle=g-1$. The denominator of $(6.52)$ can be written as:

$$
\prod_{\alpha>0}[\langle e, \alpha\rangle]=\prod_{l(\alpha)=1}^{g-1}[l(\alpha)]^{N t(\alpha)}
$$

$l(\alpha)$ is the level of $\alpha$ and $N l(\alpha)$ is the number of positive roots with the same level. For $q^{p}=1, p>g$ the $q$-dimension of the generating representations of $G$ are positive and if we consider representations in increasing values of $\langle\lambda, \theta\rangle$ we find that the $q$-dimension stays positive until $\langle\lambda, \theta\rangle=p-g$, for $\langle\alpha, \theta\rangle=p-g+1$ the $q$-dimension vanishes, and beyond this value it can be positive, negative or zero, and we begin to have null vectors, reducible but not fully reducible representations appear, etc. In analogy with $\mathrm{SU}(2)$ let $p=k+g$, the integrable (regular) irreducible representations are such that their highest weights are not in

$$
\operatorname{Im}\left(X_{\theta}^{+}\right)^{p-g+1}
$$

The first one with varishing $q$-dimension appears when $\langle\lambda, \theta\rangle=k+1$ and it is such $|\lambda\rangle=\left(X_{\theta}^{+}\right)^{k+1}|\alpha\rangle$. Hence the condition $|\lambda\rangle \notin\left(X_{\theta}^{+}\right)^{k+1}$ restricts the representations to those with $\langle\lambda, \theta\rangle \leq k$. Moreover, since $\langle\lambda, \theta\rangle \geq\langle\lambda, \alpha\rangle, \alpha>0,\left(X_{\alpha}^{-}\right)^{k+1}|\lambda\rangle=0, \forall \alpha$. This is the same condition of integrability as for Kac-Moody algebra. Proceeding as with $\mathrm{SU}(2)$ one can derive the "depth" rule" giving the fusion rules for WZW theories with group $G$ and level $k$. If $V_{\text {reg }}$ is the set of regular representations we can define the restricted tensor product

\section{$\left(V_{1} \otimes V_{2}\right)^{\prime}$}

as for $\mathrm{SU}(2)$ and the co-associativity of the co-multiplication implies the associativity if the restricted tensor product. The representation $\left(V_{1} \otimes V_{2}\right)^{\prime}$ is reducible and fully reducible. If we analyze the decomposition of $\left(V_{1} \otimes\right.$ $\left.V_{2} \otimes \ldots V_{n}\right)^{\prime}$ for regular representations, we can also read off the regular representations and the Bratelli diagram of the various ceriralizer algebras characterizing the braid representations generated by the conformal blocks associated to the decomposition of the tensor product (6.4). In the case of $\mathrm{SU}(N)$ and with all but one of the external legs in the fundamental representation we can check that one recovers the results of Wenzl ${ }^{59}$. For other groups or other representations the analysis of Wenzl has not been carried out yet and the restricted tensor product constructed here provides a rather simple way of characterizing the regular representations of all the centralizer algebras that appear in the decomposition of representations of quantum groups when $q$ is a root of unity. For $\mathrm{SU}(N)$ the representations are labels in terms of Young tableaux $\left[\lambda_{1}, \lambda_{2}, \ldots, \lambda_{N}\right], \lambda_{1} \geq \lambda_{2} \ldots$ where $\lambda_{1}$ is the number of blocks in the first row, $\lambda_{2}$ is the number of boxes in the second row etc. Then $\langle\lambda, \theta\rangle=\lambda_{1}-\lambda_{N} \leq p-g=k$ in Wenzl's notation, only diagrams of type $(N, k)$ appear (at most $N$ rows and the previous condition on the number of columns). The Bratelli diagram of the fundamental representation is easy to work out. Consider a Young tableau $Y$ with $\lambda_{1}<k, \lambda_{N}=0$. If we take the tensor product with one more copy of the fundamental representation we proceed as with standard $S U(N)$ until $\lambda_{1}-\lambda_{N}=k$. In this case the restricted tensor product eliminates the representation which could be generated by adding one more box to the first row (or any row with the same length as the first one). The arguments is easier to explain with one example. In Fig. 17 we present the Bratelli diagram for the fundamental representation of $\operatorname{SU}(3, q)$ with $q^{5}=1$ i.e, level $k=2$. 
To conclude this section we want to show that the quantum dimensions (5.38) for level $k$ WZW models with group $G$ are given by (6.52). Instead of taking the characters $\chi_{\lambda}(\tau)$ we can consider the characters with arbitrary angles in the Cartan subalgebra

$$
\chi_{\mu}(z, \tau)=\mathrm{t}_{r_{H}} q^{L_{0}-c / 24} e^{i z \cdot H}
$$

$z$ is an $r$-dimensional vector ( $r$ is the rank of $G$ ). The modular properties of $\chi_{\mu}(z, \tau)$ are the same as those of $\chi_{\mu}(\tau) .{ }^{49}$ If we take $z=g /(k+g)$ we can use the Weyl-Kac character formula to obtain

$$
\frac{\chi_{\mu}\left(\frac{e}{k+g} \mid \tau\right)}{\chi_{0}\left(\frac{Q}{k+g} \mid \tau\right)}=D_{q}(\mu) \frac{\Sigma_{\alpha \in M} e^{i \pi \tau((k+g) \alpha+\mu+e)^{2} / k+g}}{\Sigma_{\alpha \in M} e^{i \pi \tau((k+g) \alpha+\mu+e)^{2} / k+g}}
$$

where $M$ is the lattice generated by the long roots. We can take the $\tau \mapsto 0$ limit by first applying the Poisson resummation formula to the numerator and denominator of the right-hand side in $(6.55)$ to obtain:

$$
\lim _{\tau \rightarrow 0} \frac{\chi_{\mu}\left(\frac{e}{k+g} \mid \tau\right)}{\chi_{0}\left(\frac{e}{k+g} \mid \tau\right)}=D_{q}(\mu) \quad q^{k+g}=1 .
$$

Hence

$$
D_{q}(\mu)=\frac{S_{\mu 0}}{S_{00}}
$$

From (5.18) and (5.38) we conclude that the $q$-dimensions are the eigenvalues of the fusion algebra for WZW models. This agrees also with the derivation of the fusion rules based on the restricted tensor products.

It is intriguing that after the representation theory of the quantum group is restricted as indicated above, the quantum group looks closer to a finite group than to a classical or Kac-Moody group.

Before finishing this subsection we would like to make a few comments in relation with Witten's three-dimensional construction of conformal field theories. The main point in that construction is the identification of the space of blocks of the WZW theory with the physical Hilbert space of the Chern-Simons theory. Considering an $S^{2}$ section of the three-dimensional manifold and working in the temporal gauge, the physical Hilbert space for $n$ external charges in representations $R_{i}$ of the gauge group is given by ${ }^{33}$ : -

$$
X_{p h}^{R_{i} R_{3} \ldots R_{n}}=\otimes_{i=1}^{n} K^{R_{i}} /(\text { Gauss'law })=\operatorname{Inv}\left\{\otimes_{i=1}^{n} K^{R_{i}}\right\}
$$

where the $\not R_{i}$ are the representation spaces for the irreducible representations $R_{i}$ and Gauss'law is defined by the constraint:

$$
\frac{k}{8 \pi} \varepsilon^{i j} F_{i j}^{a}(x)=\sum_{k=1}^{n} \delta^{(2)}\left(x-P_{i}\right) T_{(k)}^{a}
$$

For the fundamental representation, the spaces $\mathcal{X}_{p h}^{R_{i} R_{2} \ldots R_{n}}$ define irreducible representations of the Hecke algebra $H_{n}(q), q=e^{2 x i / k+2}$. This follows from the identiscation of the space of conformal blocks with $K_{p h}$. We can use the physical Hilbert space defned above to write down a "branching rule" decomposition:

$$
\otimes_{1}^{n} X^{R}=\bigoplus_{j}\left\{K^{R \ldots R, R_{j}}\right\} \otimes K^{R_{j}}
$$

which is equivalent to equation (6.4). From the last equation and the identification of $K_{p h}$ with the space of blocks we learn that the commutant of the Hecke algebra (i.e. the quantum group) acts on $\otimes^{n} \mathcal{K}^{R}$. From the previous arguments we see that this group parametrizes the inequivalent solution to Gauss' law constraint. This connection was already foreseen in Ref. 33 .

An alternative way to get the same result is to consider invariants in the quanturn group. If we have a tensor product of $n$ representations $V^{i}$, the space of invariants is given by

$$
\operatorname{Inv}\left(\otimes_{i=1}^{n} V^{i}\right)=\left\{v \in \otimes_{i=1}^{n} V^{i} ; \varrho_{1} \otimes \varrho_{2} \ldots \varrho_{n}\left\{\Delta^{n-1}(a)\right) v=a \forall_{a} \in A\right\} .
$$

This set is nonempty only when invariant tensors can be constructed out of the representations appearing in the tensor product, and they define interaction vertices according to the fusion rules. For instance, if we work with $\mathrm{SU}(2, q), q^{3}=1$ then $\operatorname{Inv}\left(V^{1 / 2} \otimes V^{1 / 2} \otimes V^{1}\right)$ is empty. Coming back to $(6.57)$, we know that the space of invariant tensors determined by Gauss'law are the ones allowed by the fusion rules of the $S U(2)$ level $k$ WZW model. In this chapter we have seen that this is precisely what happens for $\mathrm{SU}(2, q), q=e^{2 \pi i / k+2}$. Thus we can formally identify the generators of (6.58) for $n$ charges in the representations $R_{i}$ with $\otimes_{i=1}^{n} \varrho^{R_{i}}\left(\Delta^{n-1}(a)\right)$ with a a generator of quantum $\mathrm{SU}(2)$.

\section{Explicit Examples and Modular Properties}

This section will make more concrete the arguments in Sec. 6.1. We will first show how the duality properties of WZW models follow from the 
representation theory of quantum group $\mathrm{p}^{23,25}$. Other examples are provided by the rational Gaussian models ${ }^{25}$ (Sec. 7.2). The first two subsections will deal mostly with the genus zero properties, $B$ and $F$ matrices, etc. The last subsection deals with one of the more important results obtained in the connection between quantum groups and RCFT, namely that the modular matrix $S_{i j}$ is obtained from the properties of the co-multiplication ${ }^{25}$. This will be explained in detail and we will present a proof of Verlinde's conjecture in the context of quantum groups. The argument will show quite cleary the close relation with knot theory and Witten's use of $3 \mathrm{D}$ topological field theories to understand the properties of RCFT.

\subsection{WZW models}

Although in general we do not yet know whether it is possible to associate a quantum group to any RCFT (or to a solution to the polynomial equations), in some cases it is not difficult to derive the quantum group whose representation theory reproduces the duality properties of the RCFT. Ideally one would like to find an explicit construction of the quantum algebra $A$ in terms of the generators of the chiral algebra $A$ (see Sec. 6.1). There are several ways of constructing quantum group actions on the space of blocks for $\mathrm{RCFT}^{22,24,60}$, however it is not known at present how to relate them to $k$. The previous arguments are helpful in understanding why such a construction may not be so simple. The duality properties encoded by the quantum groups correspond to rather nonlocal operations in terms of the actual blocks (as sections of a vector bundle over some moduli space), for example one has to look at analytic continuation from one region to another, etc. Hence the quantum group generators should be nonlocal functionals of the generators of $A$. We find it more appealing and easier to find the quantum group as a centralizer of the half monodromies, and then use its representation theory to express the $B, F, S, T$ matrices characterizing a solution to the polynomial equations.

As an example consider the $\mathrm{SU}(2)_{k} \mathrm{WZW}$ theory, and take $\phi=\phi_{1 / 2}$ for simplicity. This field generates the operator algebra in the sense that any other primary field $\phi_{j}, j \leq k / 2$ can be obtained in terms of the OPE of a sufficient number of $\phi$ fields. Consider the blocks in Fig. 26. The dimension of the field $\phi_{j}$ is $h_{j}=j(j+1) /(k+2)$. From the SU(2) fusion.

$$
\begin{gathered}
\phi_{1 / 2} \times \phi_{j}=\phi_{j-1 / 2}+\phi_{j+1 / 2} \quad \text { if } j \leq \frac{k-1}{2} \\
\phi_{1 / 2} \times \phi_{k / 2}=\phi_{(k-1) / 2}
\end{gathered}
$$

Then the Bratelli diagram of Fig. 28 corresponding to ordinary SU(2) will not keep on expanding indefinitely and it will truncate as shown in Fig. 29. We can characterize quite accurately the representations of the braid group furnished by the blocks $F_{\phi, i}^{(N)}$ (as in Fig. 26) from the information we have about the fusion rules (7.1) and the conformal weights $h_{j}$ of the fields $\phi_{j}$. First in the operation (6.1) we have

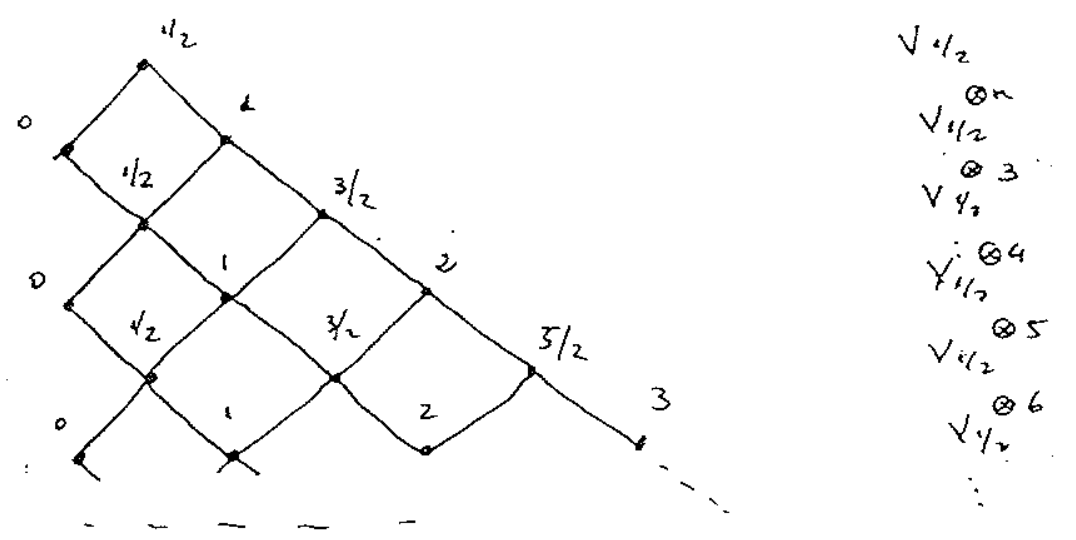

Fig. 28. Bratelli diagram for ordinary SU(2). Each row represents the spins appearing in the decomposition of $V_{1 / 2}^{N}(N=$ row number $)$, and the number of paths from the top to a given spin in the Nth row given the multiplicity of the representation.

$$
\begin{aligned}
\sigma_{12}\left(W_{j_{3}}^{j_{1} j_{2}}\right) & =(-1)^{j_{3}-j_{1}-j_{3}} q^{\left(c_{j_{3}}-c_{j_{1}}-c_{j_{3}}\right) / 2} W_{j_{3}}^{j_{2} j_{3}} \\
q & =e^{\frac{2 \pi i}{k+2}} \quad c_{l}=l(l+1) .
\end{aligned}
$$

Using the $F$ move (Fig. 4) we can diagonalize the matrix $e_{i}$ braiding the legs $i, i+1$. From (7.1) we learn that all the $\varrho_{i}$ have the same eigenvalues. By a phase redefinition these eigenvalues can be taken to be $1, q$. Therefore the matrices $\varrho_{i}$ satisfy a quadratic equation. This together with the braid relations defines the structure of a Hecke algebra of type $A_{N} ; H_{N}(q)$ :

$$
\begin{aligned}
& \varrho_{i}^{2} \quad=(1-q) \varrho_{i}+q \\
& \varrho_{i} \varrho_{i+1} \varrho_{i}=\varrho_{i+1} \varrho_{i} \varrho_{i+1} \\
& \varrho_{i} \varrho_{j} \quad=\varrho_{j} \varrho_{i} \quad|i-j| \geq 2
\end{aligned}
$$




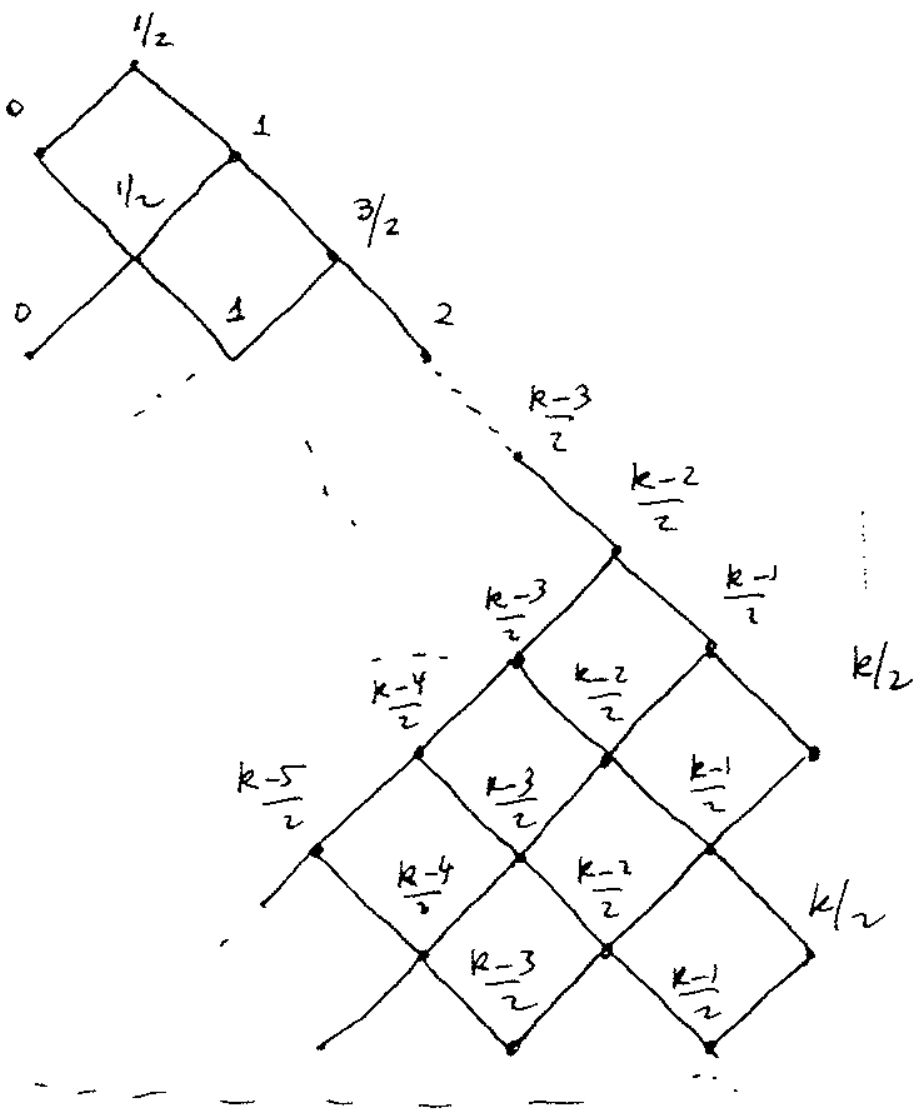

Fig. 29. Bratelli diagram for the SU(2) $k$ WZW theory.

(See also $(6.35)-(6.40))$. For generic values of $q$ the representations of $H_{N}(q)$ are in one-to-one correspondence with those of the permutation group $S_{N}$ which satisfies the same defining relation as the Hecke algebra except that $q=1$. When $q$ is a root of unity the algebra is not semisimple and to obtain a semisimple representation theory we have to consider quotients of the algebra by some ideals. Wenzl ${ }^{59}$ has studied this question in detail and has classified the necessary restrictions in the representations of $H_{N}(q)$. From the general properties of conformal theories we know that the algebra representing the action of the braid group must be effectively semisimple because the blocks provide fully reducible representations of $B_{N}$. For the blocks considered in this example the semisimple quotient of the Hecke algebra provides the representation of the braid group on the blocks. This can be seen from the fusion rules or the associated Bratelli diagram. The representations of $H_{N}(q)$ are labelled by the same Young tableau as those of $S_{N}$. In the case of $S U(2)$ we can replace the spin labels in Fig. 28 by the Young tableaux. Spin $j$ is represented by a tableau with at most two rows of $\lambda_{1}, \lambda_{2}$ boxes each such that $\lambda_{1}-\lambda_{2}=2 j$. Hence the representation of $\mathrm{SU}(2)$ appearing in the WZW model are those satisfying $\lambda_{1}-\lambda_{2}=2 k$. These are the diagrams of type $(2, k)$ in Wenzl notation and as shown in Ref. 59 they provide the *-representations of $H_{N}(q)$ when $q=e^{2 \pi i / k+2}$ (the ${ }^{*}$-representations are always semisimple algebras). The absence of tableaux with more than two rows can be written as an extra relation in the Hecke algebra demanding the three row antisymmetrizer to vanish

$$
1-\varrho_{1}-\varrho_{2}+\varrho_{1} \varrho_{2}+\varrho_{2} \varrho_{1}+\varrho_{1} \varrho_{2} \varrho_{1}=0
$$

(since we are working with SU(2) this was expected). Equations (7.2) and (7.3) are equivalent to the Temperley-Lieb-Jones algebra. See (6.35)(6.40) for more details. These algebras, known as $A_{\beta, N}$ have been extensively studied by Jones ${ }^{40,41}$ where he has classified the subfactor when $q$ is a root of unity and analyzed the ${ }^{*}$-representations. It is remarkable that many of the properties of the semisimple quotients of these algebras can be obtained directly from the theory of the WZW model whose chiral algebra is a Kac-Moody algebra. We have therefore learned that the representations of the braid group provided by the blocks $F_{\phi, i}^{N}$ in the SU $(2)_{k}$ WZW theory are in one-to-one correspondence with the ${ }^{*}$-representations of the algebra $A_{\beta, n}$. Since we know this algebra, we can ask for its centralizer in order to find the algebra $A$. As we know from Sec. 6 , the centralizer of this algebra is the quantum group $\mathrm{SU}(2, q), q=e^{2 \pi i / k+2}$ (with some restrictions on its representation theory so that it also becomes a semisimple algebra). This information can now be used to construct explicity the duality matrices $B, F$ in terms of the $q$-analogues of the $6 j$ symbols. The $F$ move (Fig. 4) corresponds to the change of basis implementing the associativity law for the composition of (quantum) angular momentum $\left(V_{1} \otimes V_{2}\right) \otimes V_{3} \cong V_{1} \otimes\left(V_{2} \otimes V_{3}\right)$. For generic values of $q$ of the $6 j$-symbols were computed and it was shown that they coincide when $q$ is a root of unity with the duality matrices for the SU(2) level $k$ WZW model. The arguments in Ref. 23 were essentially the same as those appearing here. Using Wenzl's analysis of the *-representation of the braid represen- 
tations of this conformal field theory and then finding the quantum group through the centralizer of the Temperley-Lieb-Jones algebras. The duality matrices were obtained in Ref. 6 by solving the Knizhnik-Zamolodchikov equation ${ }^{12}$ corresponding to the decoupling of null vectors of the combined Virasoro and Kac-Moody algebras. The results fo both computations (via the quantum group or via the differential equation) agree up to conjugation by a diagonal matrix. This arbitrariness is used to normalize the blocks according to one's favorite conventions. For instance the prescription in Ref. 6.

Since the previous paragraph may have been a bit too fast, we provide some more details. With the operation (6.1) we have determined that the deformation parameter of $\mathrm{SU}(2, q)$ must be a root of unity if we want to make contact with WZW models. From Sec. 6.2 however we know what are the precise conditions for the space of blocks (6.4) to provide fully reducible representations of the braid group. We have to use the modified tensor products (6.50) and (6.51). In general, we know if $q^{p}=1$, the set of regular representations $\nu_{\text {reg, }}$, contains only those representations with highest weights satisfying $\langle\lambda, \theta\rangle \leq p-g=k$. In quantum groups we can define the intertwiner

$$
K_{k}^{i j}:\left(V^{i} \otimes V^{j}\right)^{\prime} \mapsto V^{k}
$$

and the universal $R$ matrix yields the operation (6.1). As a general consequence of the properties of quantum groups ${ }^{28}$ we have

$$
K_{k}^{i j} R^{j i}=\varepsilon q^{\left(c_{k}-c_{i}-c_{j}\right) / 2} K_{k}^{j i}
$$

where $c_{i}$ is the quadratic Casimir of the classical algebra for the same representation. Hence, modulo integers we can identify the conformal dimensions of the field generating $V^{i}$ as

$$
h_{i}=\frac{c_{i}}{k+g}
$$

Notice that in (7.4) the intertwiner is defined with respect to the restricted tensor product. In this case we have a positive definite scalar product and we can define the adjoint

$$
K_{i j}^{k}: V^{k} \mapsto\left(V^{i} \otimes V^{j}\right)^{\prime}
$$
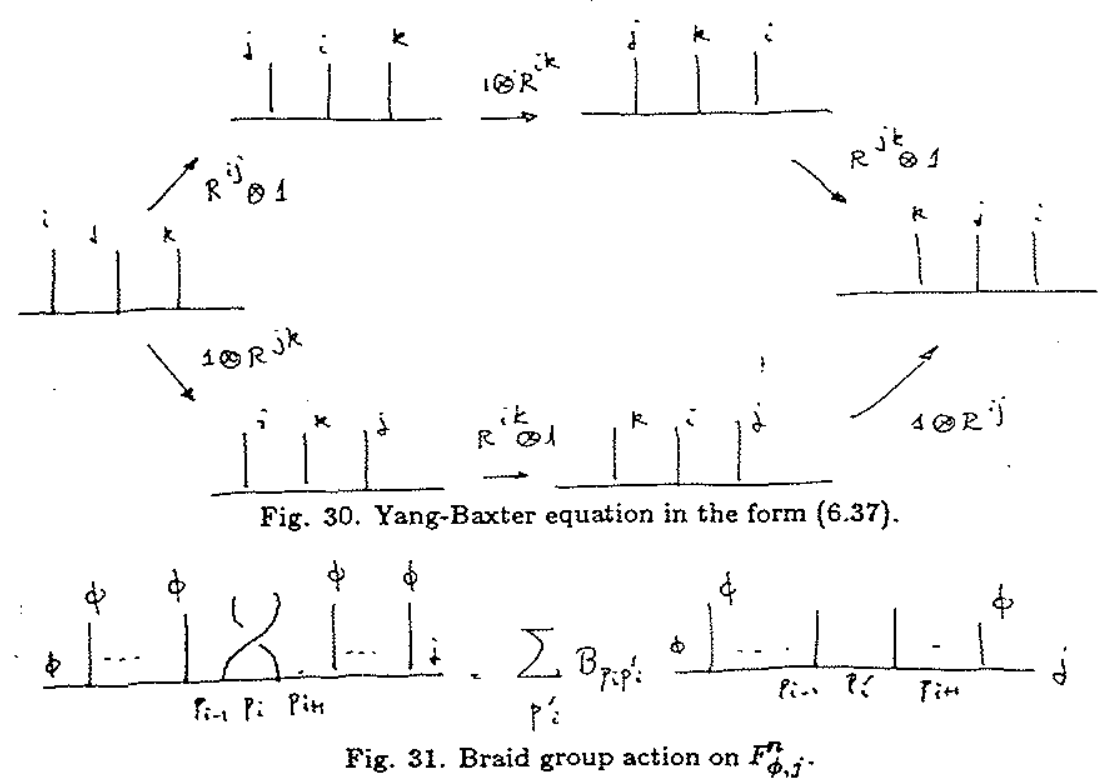

so that the orthogonality relations now become

$$
\sum_{k} K_{i j}^{k} K_{k}^{l m}=\delta_{i}^{l} \delta_{j}^{m}\left(l_{i} \otimes l_{j}\right)^{\prime}
$$

with the sum extended only over the representations appearing in the decomposition of the restricted tensor product $\left(V^{i} \otimes V^{j}\right)^{\prime}$.

The hexagon identity is a simple consequence of (6.37) which is represented graphically in Fig. 30. The explicit formulae for the braiding and fusing matrices are given in terms of the $q$-analogues of the $6 j$-symbols for $\mathrm{SU}(2)$ or their generalizations for other groups. They determine explicity* the isomorphism

$$
\left.\left(\left(V_{1} \otimes V_{2}\right)^{\prime} \otimes V_{3}\right)^{\prime} \cong V_{1} \otimes\left(V_{2} \otimes V_{3}\right)^{\prime}\right)^{\prime}
$$

The pentagon identity is a consequence of the associativity of the restricted tensor product which in turn follows from the co-associativity of the comultiplication.

As argued before, the matrices of this change of basis give the $F$ move in the space of blocks an the $B$-move is obtained from it using (6.2). For theories where the representation ring is generated by one representation 
(in Conformal Field Theory this means that there is an operator out of which all other primaries are obtained by decompositing its OPEs) the braiding and fusion matrices of more interest are those of the generating feld with arbitrary spectators. Using associativity and the fusion rules one can in principle determine the fusion and braiding matrices of other representations. In the case of $\mathrm{SU}(N)$ the $F, B$ matrices are obtained up to conjugation by a diagonal matrix using rather simple arguments and they are shown to be the same as those obtained in WZW theories by solving the Knizhnik-Zamolodchikov equation ${ }^{12}$. For level $k>1$ there are two irreducible representations appearing in the tensor product of two fundamental representations $V \otimes V, V \otimes V=V_{a}+V_{s}$ (the subscripts $s, a$ stand for $q$-symmetric and $q$-antisymmetric respectively). Using the $F$ move we diagonalize the braiding matrix of two contiguous representations and find that they have only two distinct eigenvalues. Choosing the common phase of all the braiding matrices conveniently we can make these two eigenvalues equal to $1, q=e^{2 \pi i / k+g}$ and therefore the braid generators $\varrho_{i}$ satisfy a Hecke algebra relation (6.35) together with the condition that the $N+1$ row antisymmetrizer vanishes. Both the quantum group and the WZW theory provide unitary representations of this algebra labelled by the same tableaux. This is a consequence of the relation between the Bratelli diagrams of the quantum group and its centralizer, and in the WZW theory it follows from the fusion rules. Since the basis we have chosen for the blocks have the same quantum numbers as those for the WZW theory, the matrices of these two representations must be the same up to diagonal conjugation ${ }^{23,62}$. In WZW one can determine the explicit form of the representation matrix by solving the Knizhnik-Zamolodchikov equation. In the quantum group we know that the action of the braid group on tensor products is given by

$$
\varrho\left(\sigma_{i}\right)=\varrho_{i}=1 \otimes \ldots \otimes R_{i, i+1} \otimes \ldots \otimes 1
$$

and after the tensor product is decomposed, we have an action on the blocks (see Fig. 31):

$$
\begin{array}{r}
\varrho_{i} F_{j}\left(\ldots p_{i}-1, p_{i}, p_{i}+1 \ldots\right)= \\
\sum_{p_{i}^{\prime}} B_{p_{i}, p_{i}^{\prime}}\left[\begin{array}{cc}
\phi & \phi \\
p_{i-1} & p_{i=1}
\end{array}\right] \\
F_{j}\left(\ldots p_{i-1}, p_{i}^{\prime}, p_{i+1} \ldots\right)
\end{array}
$$

in the $S U(2)$ case $\phi=\phi_{1 / 2}$. Using (6.2) and the definition of the $6 j$-symbols we obtain, ${ }^{23}$ :

$$
F_{j j^{\prime}}\left[\begin{array}{ll}
j_{2} & j_{3} \\
j_{1} & j_{4}
\end{array}\right]=\left\{\begin{array}{lll}
j_{1} & j_{2} & j \\
j_{3} & j_{4} & j^{\prime}
\end{array}\right\}
$$

$$
\begin{aligned}
B_{j j^{\prime}}\left[\begin{array}{ll}
j_{2} & j_{3} \\
j_{1} & j_{4}
\end{array}\right]= & (-1)^{j+j^{\prime}-j_{1}-j_{4}} q^{\left(c_{j_{1}}+c_{j_{4}}-c_{j}-c_{j^{\prime}}\right) / 2} \\
& \left\{\begin{array}{lll}
j_{2} & j_{1} & j \\
j_{3} & j_{4} & j^{\prime}
\end{array}\right\}
\end{aligned}
$$

where

$$
\begin{aligned}
& \left\{\begin{array}{ccc}
j_{1} & j_{2} & j_{12} \\
j_{3} & j & j_{23}
\end{array}\right\}=\Delta\left(j_{1}, j_{22}, j_{12}\right) \Delta\left(j_{3}, j, j_{12}\right) \Delta\left(j_{1}, j, j_{23}\right) \Delta\left(j_{3}, j_{2}, j_{23}\right) \times \\
& \left.\sum_{x \geq 0}(-1)^{x} \mid z+1\right] ! \frac{1}{\left[z-j_{1}-j_{2}-j_{12}\right] !\left[z-j_{3}-j-j_{12} \mid !\left[z-j_{1}-j-j_{23}\right] !\left[z-j_{3}-j_{2}-j_{23}\right] !\right.} \\
& \frac{1}{\left[j_{1}+j_{2}+j_{3}+j-z \mid !\left[j_{1}+j_{3}+j_{12}+j_{23}-z\right] !\left[j_{2}+j+j_{12}+j_{23}-z\right] !\right.} \\
& \Delta(a, b, c)=\sqrt{\frac{[-a+b+c] ![a-b+c] ![a+b-c] !}{\{a+b+c+1] !}}
\end{aligned}
$$

implementing the $B, F$ moves for SU(2) WZW theories.

To summarize, the duality properties of a RCFT contain the purely topological information characterizing a CFT. We argued in Sec. 6.1 (and exhibited explicity here) that the braiding and fusing properties of the conformal blocks can be translated into the defining properties of a QuasiTriangular Yang-Baxter algebra obtained as the centralizer of the action of the braid group on the conformal blocks. With so little information one can go quite far in the explicit computation of the duality matrices in terms of representation theoretic quantities provided by the quantum group. In a more conventional approach this information is obtained only after solving the Knizhnik-Zamalodchikov equations. It is quite remarkable that both computation differ only by conjugation with diagonal matrices (block normalization).

In Sec. 7.3 we will present the computation of the modular transformation matrix $S_{i j}$ from the quantum group point of view.

\subsection{Rational gaussian models}

As a final example we consider the rational theories at $c=1$ : consisting of a single scalar field $\phi$ taking values on a circle of radius $R=\sqrt{r / s}$ 
(where $s$ is odd). The chiral algebra is generated by the two fields $\partial \phi(z)$ and $\exp ( \pm 2 i \sqrt{r / s} \phi(z)$. For details see Ref. 57. The primary fields are vertex operators $\phi_{p}=e^{i p \phi(z)}$ where the momentum is quantized as

$$
p=\frac{k}{2 \sqrt{r s}} \quad k \in Z / 4 r s Z=Z_{4 \mathrm{rs}} .
$$

$\phi_{p}$ has conformal dimension $h_{p}=p^{2}=k^{2} / 4 r s$ and the fusion rules are

$$
\phi_{k} \times \phi_{k^{\prime}}=\phi_{k+k^{\prime}} \quad k, k^{t}, k+k^{t} \in Z_{4 r s}
$$

The quantum group description of this RCFT is generated by the operators $p, t_{ \pm}$in correspondence with $\partial \phi(z)$ and $\exp (+2 i \sqrt{r s} \phi(z)$. They satisfy the commutation relations

$$
\begin{aligned}
& {\left[p, t_{ \pm}\right]= \pm M t_{ \pm}} \\
& {\left[t_{+}, t_{-}\right]=0 .}
\end{aligned}
$$

We want to first show how one endows this algebra with the structure of a quantum group and then show that the duality properties of the rational Gaussian model are recovered from its representation theory. The Hopf algebra axioms are satisfied by the following definitions

$$
\begin{array}{lrl}
\Delta(p)=p \otimes 1+1 \otimes p & \varepsilon(p)=0 & \gamma(p)=-p \\
\Delta\left(t_{ \pm}\right)=t_{ \pm} \otimes q^{p}+q^{-p} \otimes t_{ \pm} & \varepsilon\left(t_{ \pm}\right)=0 & \gamma\left(t_{ \pm}\right)=-q^{ \pm M} t_{ \pm} .
\end{array}
$$

So far the two parameters $q, M$ are independent. The next step is to find under what conditions this Hopf algebra $Q(q, M)$ becomes a $Q$ uasi-Triangular Yang-Baxter algebra $(6.12,13)$. Take as the universal $R$-matrix the ansatz

$$
R=q^{a p \otimes p}
$$

then (6.12) and (6.13) imply the conditions

$$
q^{4 p}=q^{2 M a p}=1
$$

It is interesting to see that quasi-triangularity imposes important restrictions on the Hopf algebra $Q$. Taking $q$ as a phase we obtain the quantization conditions on the spectrum of $p$. We normalize $q^{a}$ to $e^{2 \pi i}$, which is equivalent to choosing conventions so that $h_{p}=p^{2}$. Then (7.12) leads to

$$
p=\frac{a}{4} k \quad M=\frac{2 \nu_{0}}{a} \quad k, \nu_{0} \in Z
$$

Since $t_{+}$changes the eigenvalue of $p$ by $M$ units to obtain a representation of the algebra we must have that $p+M$ also takes the form $\frac{a}{4} k^{\prime}$, hence

$$
a=2 \sqrt{\frac{2 \nu_{0}}{N}} N \in Z
$$

All this together yields the quantization conditions

$$
p=\frac{k}{2 \sqrt{r s}} N=2 \nu_{0} r s
$$

To obtain a rational representation theory note first that the irreducible representations of $Q$ are labelled by the eigenstates of $p,|p\rangle$ with $p=(k+$ $m N) / 2 \sqrt{r s}, m \in Z$ obtained from $|k / 2 \sqrt{r s}\rangle$ by repeated action of $t_{ \pm}$. Since the braiding properties of two elements in the same representation of the chiral algebra are the same we find that for a state in $\left\{\left|p_{1}\right\rangle\right\}$ and a state in $\left\{\left|p_{2}\right\rangle\right\}$ the braiding is

$$
e^{2 \pi i p_{1} p_{2}}=e^{2 \pi i k_{1} k_{2} / 4 r s} e^{i \pi\left(m_{2} k_{2}+m_{2} k_{1}\right) \nu_{0}}
$$

implying that to have a consistent representation theory, $\nu_{0}$ must be an even integer. In fact there is no loss of generality if we choose $\nu_{0}=2$. If $\nu_{0} \geq 2$ we can always construct other operators $t_{ \pm}^{\prime}$ which are "roots" of $t_{ \pm}$and for them $\nu_{0}=2$. This implies that $k \in Z_{4 r s}$. When we enlarge the representations of $p$ as indicated in order to label them only with integers mod. 4rs we have to make sure that $t_{ \pm}$is still compatible with the modding and the similarly for the co-multiplication. The fusion rules are very easy in this case. Assuming the Clebsch-Gordan rule

$$
\left|p_{1}\right\rangle\left|p_{2}\right\rangle=\left|p_{1}+p_{2}\right\rangle
$$

we have

$$
t_{+}|p\rangle=N_{p}|p+M\rangle
$$


From the co-multiplication we can derive the compatibility condition

$$
N_{p_{1}} q^{p_{2}}+N_{p_{2}} q^{-p_{1}}=N_{p_{1}+p_{2}}
$$

solved by

$$
N_{p}=\sin \frac{k \pi}{2} \quad p=\frac{a}{4} k \quad N_{p+N}=N_{p}
$$

and in the reduced theory $t_{ \pm}$does not act as the identity

$$
t_{ \pm}|k\rangle=\sin \frac{k \pi}{2}|k\rangle \text {. }
$$

$t$ cannot act as the identity because $\Delta(1)=1 \otimes 1$ and it would not be compatible with the reduction. Therefore the rational Gaussian models can be recovered (or rather their duality properties) by looking at the quantum group $Q$ and imposing a rationality condition on its representation ring (to have only a finite number on representations).

\subsection{Modular properties}

We now construct the matrix $S$ using only quantum group information. Using the relation between quantum groups, RCFT and link invariants detailed in Sec. 9 we will show that our representation of $S$ is as expected from the topological Chern-Simons theory given by the expectation value of two linked Wilson lines on $S^{3}$

From the work of Moore and Seiberg ${ }^{16,17,18}$ we know we can represent the Verlinde operators ${ }^{15}$ in terms of the duality matrices. In particular, the action of the operators associated to the a-cycle on the characters is diagonal and it is given by the eigenvalues of the fusion matrices $\lambda_{k}^{(i)}$. The precise relation with the duality matrices is

$$
\begin{aligned}
\lambda_{k}^{(j)} & =\frac{1}{F_{k}} \sum_{p} B_{o p}\left[\begin{array}{cc}
k & \bar{j} \\
k & j
\end{array}\right](+) B_{p 0}\left[\begin{array}{ll}
\bar{j} & k \\
k & j
\end{array}\right](+) \\
F_{k} & =\varepsilon_{k \bar{k}}^{0} F_{00}\left[\begin{array}{ll}
k & \bar{k} \\
k & k
\end{array}\right]
\end{aligned}
$$

where the line over an index refers to the conjugate field. (The + argument in the braiding matrices indicates that we are braiding in the positive sense, the $i+1$-st strand goes over the $i$ th strand.) The Verlinde operator acts on characters according to

$$
\phi_{k}(a) \chi_{j}=\lambda_{k}^{(j)} \chi_{j}
$$

and it can be described as inserting the identity operator factorized as $k, \bar{k}$ on the block in Fig. 20, then we braid the field $k$ around $j$. These operations result in (7.15). From (6.2) we can write (7.15) as

$$
\lambda_{k}^{(j)}=\frac{1}{F_{k}} \sum_{p} \varepsilon_{j j}^{0} \varepsilon_{p j}^{k} \varepsilon_{j k}^{p} e^{2 \pi i\left(h_{p}-h_{j}-h_{k}\right)} F_{o p}\left[\begin{array}{cc}
k & j \\
k & \bar{j}
\end{array}\right] F_{p o}\left[\begin{array}{ll}
\bar{j} & j \\
k & k
\end{array}\right] .
$$

It is easy to show as a consequence of the pentagon identity that

$$
\frac{S_{j k}}{S_{O O}}=\sum_{p} e^{2 \pi i\left(h_{p}-h_{j}-h_{k}\right)} \frac{S_{O p}}{S_{O O}} N_{j k}^{p}
$$

In the case of $\mathrm{SU}(2)$ we have

$$
S_{j l}=\sqrt{\frac{2}{k+2}} \sin \frac{\pi(2 j+1)(2 l+1)}{k+2}
$$

and (7.18) implies the following identity between $q$-numbers

$$
[(2 j+1)(2 k+1)]=\sum_{p} q^{c_{p}-c_{j}-c_{k}} N_{j k}^{p}[2 p+1] \quad c_{j}=j(j+1)
$$

This relation is certainly different from the addition formula of $q$-dimensions

$$
[(2 j+1)][(2 k+1)]=\sum_{p} N_{j k}^{p}[2 p+1]
$$

which is a particular case of the Verlinde conjecture (see Sec. 5 for details).

In the quantum group there is a useful relation between the trace of the comultiplication $\Delta(a)$ in the tensor product representation $j \otimes k$ and the trace of $a$ in the representations appearing in the decomposition of the tensor product:

$$
\operatorname{tr}_{j \otimes k} \Delta(a)=\sum_{p} N_{j k}^{p} \operatorname{tr}_{p}(a)
$$

where

$$
\operatorname{tr}_{j \otimes k} \Delta(a)=\operatorname{tr} e^{j} \otimes e^{k}(\Delta(a))
$$

and $\varrho^{j}$ is the map from the quantum group $A$ to the representation $V^{j}$. Using (6.3) one easily derives (7.20) from the orthogonality properties of 
the quantum Clebsch-Gordan coefficients. As a trivial example from (7.20) and the comultiplication

$$
\Delta\left(q^{H / 2}\right)=q^{H / 2} \otimes q^{H / 2}
$$

we obtain (7.19). In any Quasi-Triangular Yang-Baxter algebra there is always a distinguished element $u^{28}$ defined in terms of the $R$-matrix and the antipode by

$$
\begin{aligned}
u & =m(\gamma \circ i d)(\sigma(R)) \\
u^{-1} & =m\left(i d \circ \gamma^{2}\right)(\sigma(R))
\end{aligned}
$$

where $m$ is the multiplication in $A$ and we have used the notation in Sec. 6 . The element $u$ has the properties

$$
u a u^{-1}=\gamma^{2}(a) \quad \forall a \in A
$$

when $A=\mathrm{SU}(2, q)$, $u$ and $u^{-1}$ are given in the representation $j$ by

$$
e^{j}(u)=q^{-c_{j}} q^{H / 2} \quad e^{j}\left(u^{-1}\right)=q^{c_{3}} q^{-H / 2}
$$

so that

$$
\operatorname{tr}_{j} u=q^{-c_{j}}\{2 j+1] \quad \operatorname{tr}_{j} u^{-1}=q^{c_{j}}[2 j+1] .
$$

Since the construction of $\lambda_{k}^{(j)}$ follows from the polynomial equations directly, the relation (7.18) has a very nice interpretation in the quantum group (for the time being we consider $S U(2)$ ):

$$
\frac{S_{j k}}{S_{00}}=q^{-c_{j}-c_{k}} \operatorname{tr}_{j \otimes k} \Delta\left(u^{-1}\right)=q^{c_{j}+c_{k}} \operatorname{tr}_{j \otimes k} \Delta(u)
$$

the element $u$ can be identified with the remnant of the Virasoro algebra. The comultiplication rule for the element $u$ is ${ }^{29}$

$$
\begin{aligned}
& \Delta(u)=R^{-1} \sigma\left(R^{-1}\right)(u \otimes v) \\
& \Delta\left(u^{-1}\right)=\sigma(R) R\left(u^{-1} \otimes u^{-1}\right) .
\end{aligned}
$$

Finally we find the complete form of the matrix $S$ in the quantum group ${ }^{a}$

$$
\begin{aligned}
\frac{S_{j k}}{S_{00}} & =q^{-c_{j}-c_{k}} \operatorname{tr}_{j \otimes k} \sigma(R) R\left(u^{-1} \otimes u^{-1}\right) \\
& =q^{c_{j}+c_{k}} \operatorname{tr}_{j \otimes k} R^{-1} \sigma\left(R^{-1}\right)(u \otimes u) .
\end{aligned}
$$

${ }^{a}$ In the limit $q \rightarrow 1, \sigma(R) R_{\leftrightarrow 1 \otimes 1}$, this is the condition for a quasi-triangular YangBaxter algebra to be also triangular [27]. Hence the classical limit of a quantum group is a triangular Yang-Baxter algebra.
We can make contact with the Chern-Simons theory ${ }^{14}$. We know from (6.42) that the quantum group comes endowed with a trace with the Markov property which can be used to define link invariants (see Sec. 9 for more details). As in SU(2), $u$ is propotional in general to $q^{Q}$, hence

$$
(u \otimes u) R=R(u \otimes u) \text {. }
$$

To understand what link we relate with $S$ by (7.27), we recall that every link or knot can be obtained by closing a braid (see Sec. 9). Since we have a representation of the braid group whose action is defined in terms of the matrix $R$, we see that the operation $\sigma(R) R$ used in the definition of $S$ is the braid in Fig. 32a. Taking traces is like closing the braid (identifying the end points of the braid in Fig. 32a to get Fig. 32b) and we prove directly that the modular matrix $S$ is a link invariant associated to Fig. 32. In the Chern-Simons theory this conclusion is obtained by doing surgery on the trivial link (two unknotted unlinked circles on $S^{2} \times S^{1}$ ) according to the operation $S$. In other words, in the Chern-Simons theory $S_{j k}$ is the expectation value in $S^{3}$ of two linked Wilson lines

$$
S_{j k}=Z\left(S^{3}, L\left(R_{j}, R_{k}\right)\right)
$$

(see Ref. 33 for more details). In the quantum group this is interpretation is a consequence of the co-multiplication rule for the distinguished element u.

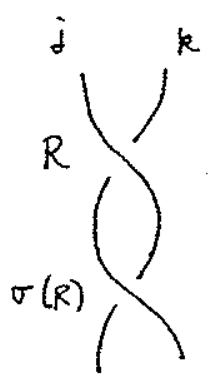

(a)

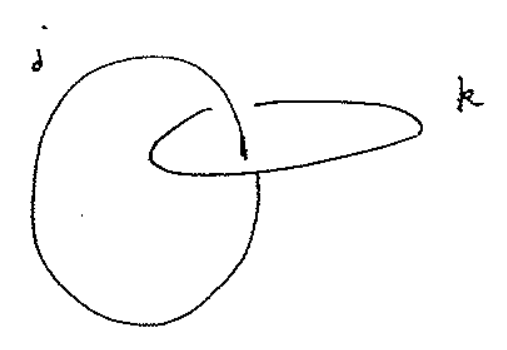

(b)
Fig. 32. Link representation of the matrix $\mathrm{S}$.

We can now proceed along the argument presented in Ref. 33 to prove the Verlinde conjecture. Since here we do not have a functional integral 


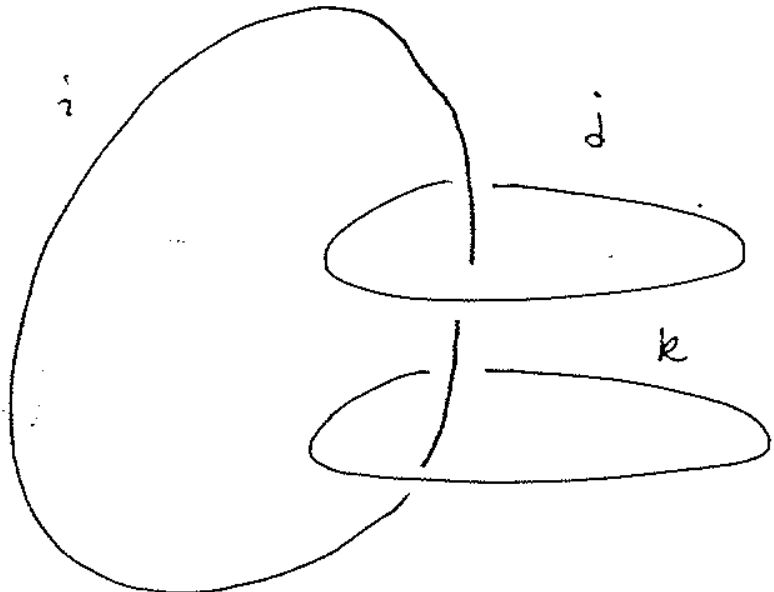

Fig. 33. Link used in the proof of the Verlinde conjecture.

interpretation (only duality properties) the proof will rely on the defining properties of a quantum group. Consider the link in $S^{3}$ in Fig. 33. It can be represented by the braid in Fig. 34 which as a representation in terms of traces is

$$
\begin{aligned}
W_{i j k} & =\operatorname{tr}_{i \otimes j \otimes k}\left(\sigma\left(R_{12}\right) \sigma\left(R_{13}\right) R_{13} R_{12} q^{-H / 2} \otimes q^{-H / 2} \otimes q^{-H / 2}\right) \\
& \equiv \operatorname{tr}_{i \otimes j \otimes k}^{M}\left(\sigma\left(R_{12}\right) \sigma\left(R_{13}\right) R_{13} R_{12}\right)
\end{aligned}
$$

where in the last step we use the definition of the Markov trace (6.24c). For simplicity we are presenting the argument in detail for SU(2). The generalization to more complicated cases is straightforward. To prove Verlinde's conjecture we compute (7.28) in two ways. First we use the defining properties of a quantum group:

$$
\begin{aligned}
& (1 \otimes \Delta) R=R_{13} R_{12} \\
& (1 \otimes \Delta) \sigma(R)=\sigma(R)_{12} \sigma(R)_{13}
\end{aligned}
$$

to write

$$
W_{i j k}=\operatorname{tr}_{i \otimes j \otimes k}(1 \otimes \Delta) \sigma(R) R\left(q^{-H / 2} \otimes q^{-H / 2}\right) .
$$

Using (7.20) we and (7.27) we obtain

$$
W_{i j k}=\sum_{m} N_{j k}^{m} \operatorname{tr}_{i \otimes m} \sigma(R) R\left(q^{-H / 2} \otimes q^{-H / 2}\right)=\sum_{m} N_{j k}^{m} \frac{S_{m i}}{S_{00}} .
$$

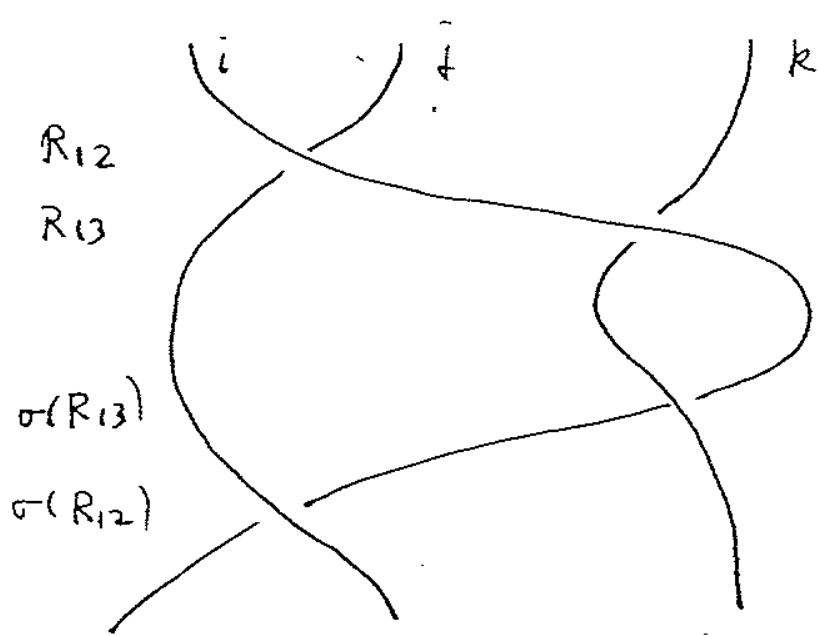

Fig. 34. Braid representation of the link in Fig. 33.

On the other hand the trace (7.28) has the Markov property (6.42c) and next section) and for the braiding we performed in Fig. 34 we have

$$
\begin{aligned}
W_{i j k} & =\frac{\left(\operatorname{tr}_{i \otimes j}^{M} \sigma(R) R\right)\left(\operatorname{tr}_{i \otimes k}^{M} \sigma(R) R\right)}{S_{0 i} / S_{00}} \\
& =\frac{S_{i j} S_{j k}}{S_{00} S_{0 i}} .
\end{aligned}
$$

From (7.29) and (7.30) we obtain the proof of Verlinde's conjecture:

$$
\frac{S_{i j} S_{i k}}{S_{0 i}}=\sum_{m} N_{j k}^{m} S_{m i} .
$$

Since $S$ is obviously symmetric and it diagonalizes the fusion rules, it follows from the general arguments of Sec. 2 that $S^{2}=C$ as expected. This now leads to all the formula in (5.18). Although for simplicity we have carried out the argument as though the representations were self-conjugate, the formulae derived hold in the general case. Using (5.18), in particular $S^{2}=C, S^{*}=S^{-1}=S C=C S$ we can rewrite $(7.18)$ as

$$
S_{i}^{j}=\sum_{k} N_{k i}^{j} e^{2 \pi i\left(h_{i}+h_{j}-h_{k}\right)} S_{0}^{k}
$$

With respect to the other defining relation of the modular group $(S T)^{3}=C$, it can be rewritten as

$$
T S T S T=S .
$$


Although this equation seems to involve many conditions on the RCFT, it is equivalent to a single equation. Using $(5.18),(7.18)$ and $(7.32)$ one can show that (7.33) is equivalent to

$$
e^{2 \pi i h_{j}} S_{0}^{j}=e^{i \pi c / 4} \sum_{k} e^{2 \pi i h_{k}} S_{0}^{k} S_{k}^{j}
$$

This suggests the definition of a quantum character as

$$
\chi_{i}(q)=e^{2 \pi i h_{i}-i \pi c / 8} \frac{S_{0}^{i}}{S_{0}^{0}}
$$

and (7.34) expresses the relation between the character with argument $q^{-1}$ and the characters with argument $q$. More generally this is a propety of the quantum characters under charge conjugation, and it should be related to the completeness of the set of rational representations of the quantum group. For quantum groups coming from deformations of classical groups (7.34) holds as one can see from explicit computations, it is also quite easy to verify this relation for Gaussian models. In general, however, an argument similar to the one for $S$ in terms of should ensure this transformation property of quantum characters, although at present this general argument is missing. It seems likely that the Markov property of the trace and its relation to knot and link invariants should provide again the steps to complete the argument in general.

This concludes the proof that quantum groups provide solutions to the polynomial equations. It should be stressed again that the quantum group comes endowed with a Markov trace, and that this is an important property with respect to the relation with knot invariants and also with respect to the partial reconstruction of the space of conformal blocks.

The reconstruction problem in RCFT is to find the extra conditions that determine when a particular representation space of the duality transformations (a solution of the polynomial equations) can be interpreted as the space of blocks of a RCFT. The quantum group approach is a first step in this direction. As we have seen we get the quantum group directly from the duality properties of the conformal theory. What we see at this point is that RCFT are always associated with $C^{*}$-irreducible representations of the duality transformations. If these $C^{*}$-representations are $\mathrm{II}_{1}$-factors i.e.

2 they admit a normalized finite trace then we will be able to identify the space of conformal blocks with the Hilbert space obtained by completion of the duality $C^{*}$-algebra with respect to the scalar product defined by the trace $\left(\langle x \mid y\rangle=\operatorname{tr}\left(x^{*} y\right)\right)$. The extra physical requirement that naturally appears in the quantum group approach is that the conformal theories are in one-to-one correspondence with solutions of the polynomial equations such that:

1) They define irreducible $C^{*}$-representations of the duality transformations, and

2) They are equipped with a normalized finite trace satisfying the Markov property (see Sec. 6 and 9). This Markov property which holds under very general circumstances is at the root of the connection between Conformal Field Theory and three-dimensional Topological Field Theories. In general if one begins with a solution to the polynomial equations some of the steps in the previous paragraphs establishing the relation with quantum groups are missing. For instance, in general we will not get the spaces $V_{i}$ themselves or even their real dimensions, but only their q-dimensions (5.38). In this cases more powerful techniques are required. It is reasonable to expect that an extension of the results of Deligne quoted in Ref. 18 concerning the reconstruction of classical groups may yield ultimately that to any solution of the polynomial equations with some extra condition about the possible $q$-dimensions can be given in terms of quantum groups. In the next sections we will look in more detail into the structure of quantum groups and show that those groups coming from classical groups do indeed satisfy the polynomial equations and look also into the general situation.

\section{Solvable Lattice Models and Conformal Field Theories \\ 8.1 Vertex models}

Following Baxter ${ }^{34,36}$ we will define a vertex model on a generic lattice $\mathcal{L}$. The lattice is defined by the condition that each site must be the endpoint of four edges, where edges are defined as the line segments between sites. These lattices can always be colored in black and white in such a way that two plaqnettes with the same color have no edge in common (Fig. 35). The statistical model on the lattice $\mathcal{L}$ is defined by associating three different interaction coeffcients $K_{i}, K_{i}^{l}, K^{n}{ }_{i}$ to each site and spin variables $\sigma_{e}= \pm 1$ to each face or plaqnette. The Boltzmann weight for a generic site $i$ is (Fig. 36):

$$
\exp \left[K_{i} \sigma_{p} \sigma_{e}+K_{i}^{\prime} \sigma_{g} \sigma_{m}+K^{n_{i}} \sigma_{p} \sigma_{q} \sigma_{e} \sigma_{m}\right]
$$




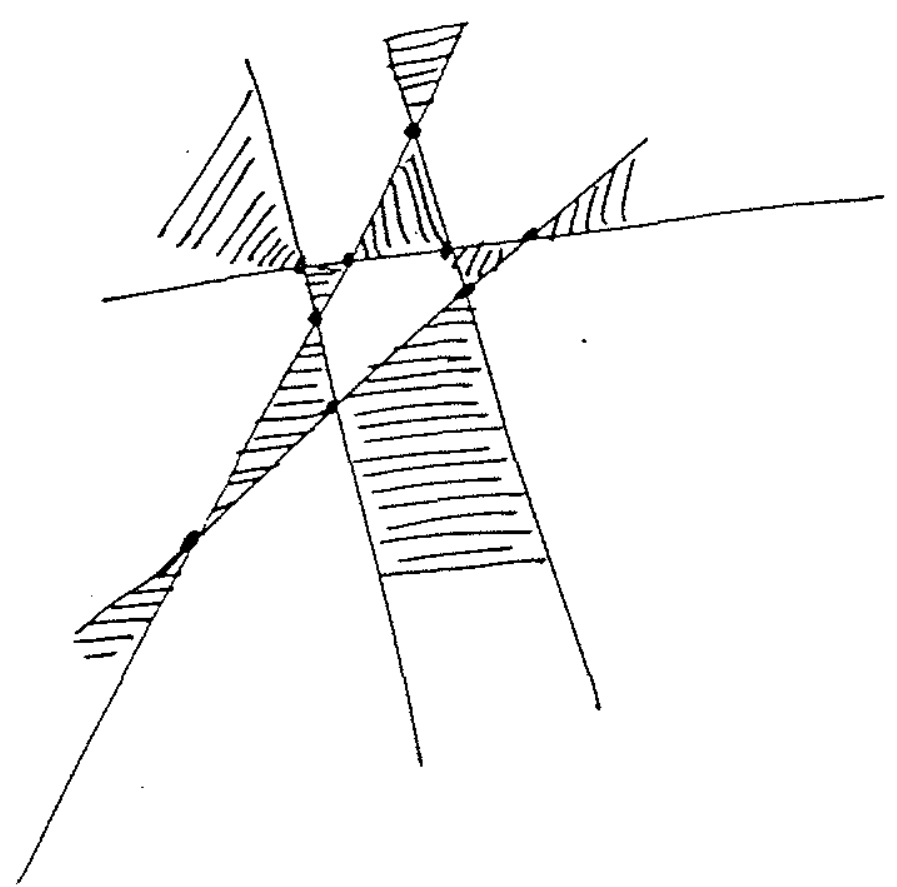

Fig. 35.

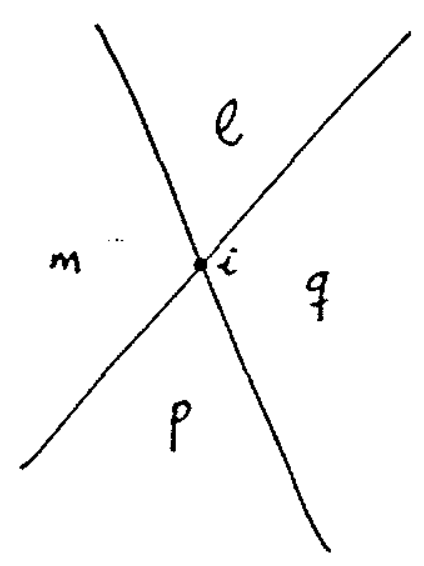

Fig. 36.

Once we have colored our lattice we can describe the different spin configurations on the plaquettes by drawing arrows on the edges of the lattice. In fact for each site we have 16 possible choices for the surrounding spins.
These configurations can be divided in two sets of 8 , where one can be obtained from the other by reversing all the spins. Notice that reversing all the surrounding spins does not change the value of the Boltzmann weight (8.1). Now we can introduce arrows on the edges of the lattice by the rule:

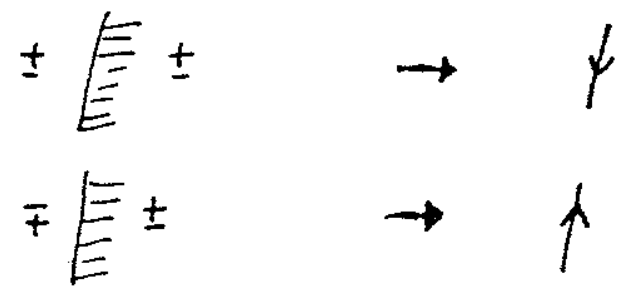

Using $(R)$ the eight different configurations are associated to the following vertices:

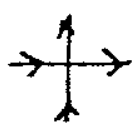

(1)

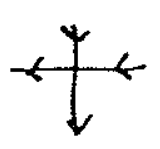

$(2)$

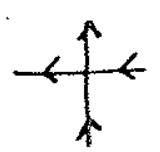

(3)

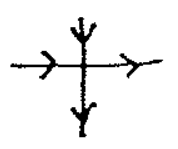

(4)

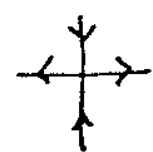

(5)

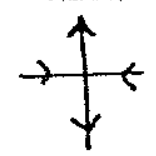

(6)

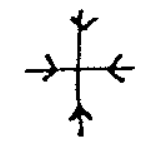

(7)

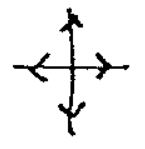

(8)
The Boltzmann weights of the eight-vertices can be obtained from (8.1). It is easy to see that they satisfy the following relations:

$$
\begin{aligned}
& w_{1}=w_{2}=\exp \left(K_{i}-K_{i}^{\prime}-K^{m}{ }_{i}\right) \\
& w_{3}=w_{4}=\exp \left(-K_{i}+K_{i}^{\prime}-K^{n}{ }_{i}\right) \\
& w_{5}=w_{6}=\exp \left(K_{i}+K_{i}^{\prime}+K^{\prime}{ }_{i}\right) \\
& w_{7}=w_{8}=\exp \left(-K_{i}-K_{i}^{\prime}+K^{n}{ }_{i}\right)
\end{aligned}
$$

Therefore we have, in principle, four independent variables for each site of the lattice. To solve the model we will consider first the case of a regular lattice and the same interaction coefficients for all sites. 
8.1.1 Regular lattice

For the Boltzmann weights we will introduce the notation $W(i, j, k, l)$ where $i, j, k, l$ are \pm 1 and refer to the directions of the arrows. The partition function of the model can be defined in terms of the transfer matrix:

$$
\mathrm{Z}=\operatorname{Tr}\left(T^{n}\right)
$$

where $n$ is the number of rows of the regular lattice. In order to define the transfer matrix consider two consecutive rows and denote by $\alpha=$ $\left(\alpha_{1} \alpha_{2} \ldots \alpha_{m}\right)$ the configuration of vertical arrows and by $\lambda=\left(\lambda_{1} \lambda_{2} \ldots \lambda_{m}\right)$ the configuration of horizontal arrows (see Fig. 37). The transfer matrix is then defined by:

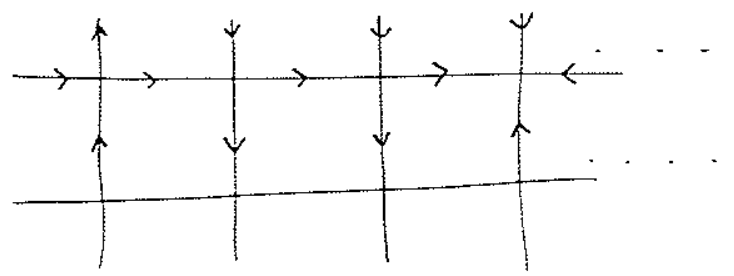

$$
\begin{aligned}
& \alpha=(\uparrow, \psi, \psi, \uparrow \ldots .) \quad \lambda=(\rightarrow, \rightarrow, \rightarrow, \rightarrow, \leftarrow, \cdots) \\
& \beta=(\uparrow, \psi, \psi, \psi \ldots .,
\end{aligned}
$$

Fig. 37.

$$
T_{\alpha \mid \beta}=\sum_{\lambda^{\prime} s} w\left(\lambda_{1}, \alpha_{1}, \lambda_{2}, \beta_{1}\right) w\left(\lambda_{2}, \alpha_{2}, \lambda_{3}, \beta_{2}\right) \ldots w\left(\lambda_{m} \alpha_{m} \lambda, \beta_{m}\right)
$$

where the sum is over all possible configurations of horizontal arrows. To solve the model is equivalent to diagonalizing the transfer matrix. Notice that the transfer matrix plays the role of the Hamiltonian and therefore to diagonalize $T$ will be equivalent to finding an infnite set of conserved quantities. To find the conserved quantities we look for a new set of Boltzmann weights. Let us say $w^{\prime}(i, j, k, l)$ such that the new transfer matrix $T^{\prime}$
Using (8.5) we can write:

$$
\left(T T^{\prime}\right)_{\alpha \beta}=\sum_{\mu, n u} \prod_{i}\left(\sum_{\gamma} w\left(\mu_{i} \alpha_{i} \mu_{i+1} \gamma_{i}\right) w^{\prime}\left(\nu_{i} \gamma_{i} \nu_{i+1} \beta_{i}\right)\right)
$$

Following Ref. 64 we can write (8.7) as:

$$
\left(T T^{\prime}\right)_{\alpha \beta}=\operatorname{Tr}\left\{\prod_{i} S\left(\alpha_{i} \beta_{i}\right)\right\}
$$

with:

$$
\left[S\left(\alpha_{i} \beta_{i}\right)\right]_{\mu \nu / \mu^{\prime} \nu^{\prime}}=\sum_{\gamma^{\prime}} w\left(\mu \alpha_{i} \mu^{\prime} \gamma\right) w^{\prime}\left(\nu \nu^{\prime} \beta_{i}\right) .
$$

To get (8.6) we require the existence of a matrix $Q$ such that:

$$
S^{\prime}(\alpha, \beta)=Q S(\alpha, \beta) Q^{-1}
$$

Using (8.9) we can write (8.10) as:

$$
\begin{aligned}
& \sum_{\gamma \mu^{n} \nu^{\eta}} w\left(\mu, \alpha, \mu^{»}, \gamma\right) w^{\prime}\left(\nu, \gamma, \nu^{\eta}, \beta\right) w^{n}\left(\nu^{\eta}, \mu^{\eta}, \mu^{\prime}, \nu^{\prime}\right) \\
= & \sum_{\gamma \mu^{n} \nu^{\eta}} w^{\eta}\left(\nu, \mu, \mu^{\eta}, \nu^{»}\right) w^{\prime}\left(\mu^{\eta}, \alpha, \mu^{\prime}, \gamma\right) w\left(\nu^{\eta}, \gamma, \nu^{\prime}, \beta\right)
\end{aligned}
$$

where we denote by $w^{n}$ the elements of the matrix $Q$ satisfying Eq. (8.10). These elements can be considered as a new set of boltzmann weights. Equation (8.11) is known as the Yang-Baxter equation for vertex models. A soIution to Eq. (8.11) can be obtained using the factorized $S$-matrix models in Ref. 65. Defining the $S$-matrix by:

$$
\left\{\mu ; \theta, \alpha ; \theta^{\prime}\right\rangle=S_{\mu \alpha ; \mu^{\mathrm{r}} \gamma}\left(\theta-\theta^{\prime}\right) \quad\left|\mu^{n} ; \theta, \gamma \theta^{\prime}\right\rangle
$$

where we interpret $\mu, \alpha, \gamma$ as internal quantum numbers and $\theta$ as related to the momentum by:

$$
P^{0}=\operatorname{sh} \theta \quad p=\operatorname{coh} \theta .
$$

The factorization condition for three particle scattering is ${ }^{64}$ :

$$
\begin{aligned}
& S_{\mu \alpha ; \mu^{\prime \prime \gamma} \gamma}\left(\theta_{1}-\theta_{2}\right) S_{\mu \gamma ; \mu^{\prime \prime} \beta}\left(\theta_{2}-\theta_{3}\right) S_{\nu^{\mu} \mu^{n} ; \mu^{\prime} \nu^{\prime}}\left(\theta_{2}-\theta_{3}\right) \\
= & S_{\nu \mu ; \mu^{\prime \prime} \nu^{\prime}}^{\prime}\left(\theta_{2}-\theta_{3}\right) S_{\mu " \alpha ; \mu^{\prime} \gamma}\left(\theta_{1}-\theta_{3}\right) S_{\nu^{\prime \prime} \gamma ; \nu^{\prime} \beta}\left(\theta_{1}-\theta_{2}\right)
\end{aligned}
$$

$$
\left(T T^{\prime}\right)_{\alpha \beta}=\left(T^{\prime} T\right)_{\alpha \beta}
$$


which coincides with Eq. (8.11) if we identify

$$
\begin{aligned}
& w\left(\mu \alpha \mu^{n} \gamma\right)=S_{\mu \alpha ; \mu^{n} \gamma}\left(\theta_{1}-\theta_{2}\right) \\
& w^{\prime}\left(\nu \gamma \nu^{\eta} \beta\right)=S_{\nu \gamma ; \mu^{n} \beta}\left(\theta_{1}-\theta_{3}\right) \\
& w^{n}\left(\nu^{n} \mu^{n} \mu^{\prime} \nu^{\prime}\right)=S_{\nu^{n} \mu^{n} ; \mu^{\prime} \nu^{\prime}}\left(\theta_{2}-\theta_{3}\right) .
\end{aligned}
$$

The graphic representation of Eq. (8.14) is given in Fig. 38. The solution (8.15) to (8.11) gives us a one-parameter family of Boltzmann weights, where the parameter can be identifed with the rapidity of the imaginary scattering process (8.12). In general we can write this one-parameter family of Boltzmann weights as $w(i, j, k, l ; u)$. This defines a $u$-dependent transfer matrix $T(u)$ and Eq. (8.6) becomes:

$$
[T(u), T(v)]=0 .
$$
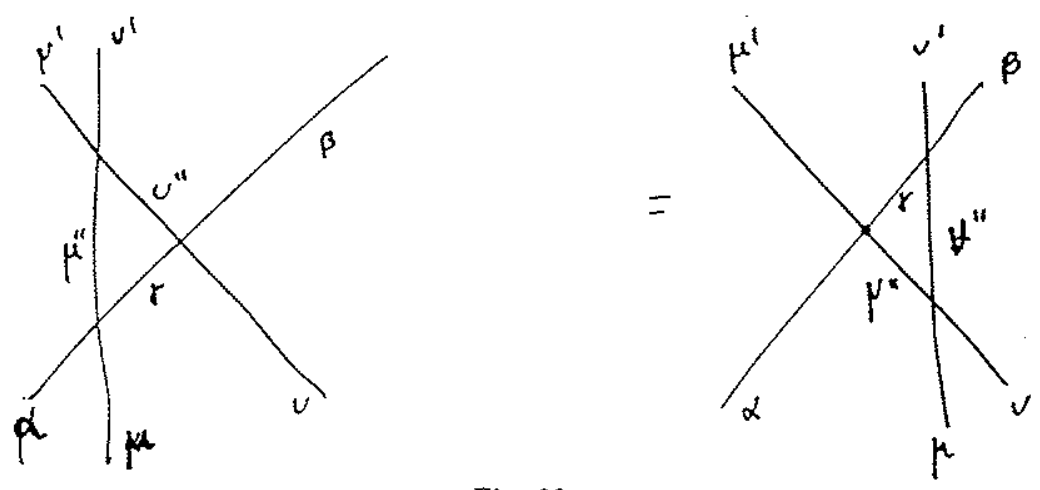

Fig. 38.

Equations (8.11) and (8.14) are typical examples of the quantum YangBaxter equation. In fact the Boltzmann weights for the eight-vertex model we are considering can be interpreted as the components of a $4 \times 4$ matrix depending on the parameter $u$. Moreover using (8.2) and (8.3) we can write:

$$
w(i j k l ; u)=\sum_{\mu, \nu} R^{\mu \nu}(u)\left(\sigma^{\mu}\right)_{i j} \otimes\left(\sigma^{\nu}\right)_{k l}
$$

where the $\sigma$ 's are the Pauli basis on the space of $2 \times 2$ matrices. Using the representation (8.17) Eq. (8.11) becomes:

$$
R^{(1,2)}(u) R^{(13)}(u+v) R^{(2,3)}(v)=R^{(2,3)}(v) R^{(1,3)}(u+v) R^{(1,2)}(u)
$$

where

$$
R^{(1,3)}(u+v)=\sum_{\mu, \nu} R^{\mu \nu}(u+v)\left(\sigma^{\mu}\right) \otimes 1 \otimes\left(\sigma^{\nu}\right)
$$

etc. Notice that (8.18) is simply (8.14) with the upper indices indicating the particles that enter in the scattering process and where we have changed variables: $\left(\theta_{1}-\theta_{2}\right)=u,\left(\theta_{1}-\theta_{3}\right)=u+v$ and $\left(\theta_{2}-\theta_{3}\right)=v$.

We now come back to the more general problem of an irregular lattice and different interaction coefficient on the sites of the lattice.

\subsubsection{Nonregular lattice. Z-invariance}

The question we want to investigate is under what conditions on the interaction coefficients can we solve a vertex model on a generic lattice. The solution to this problem will provide a geometric interpretation of the YangBaxter equation. Consider two lattices as in Fig. 39. They differ only by the translation of the line AC. The first thing we will study is when these two lattice are physically equivalent. We assume that the interaction coefficients on both lattices are the same. The Boltzmann weights for the triangles in (Fig. 40) are:
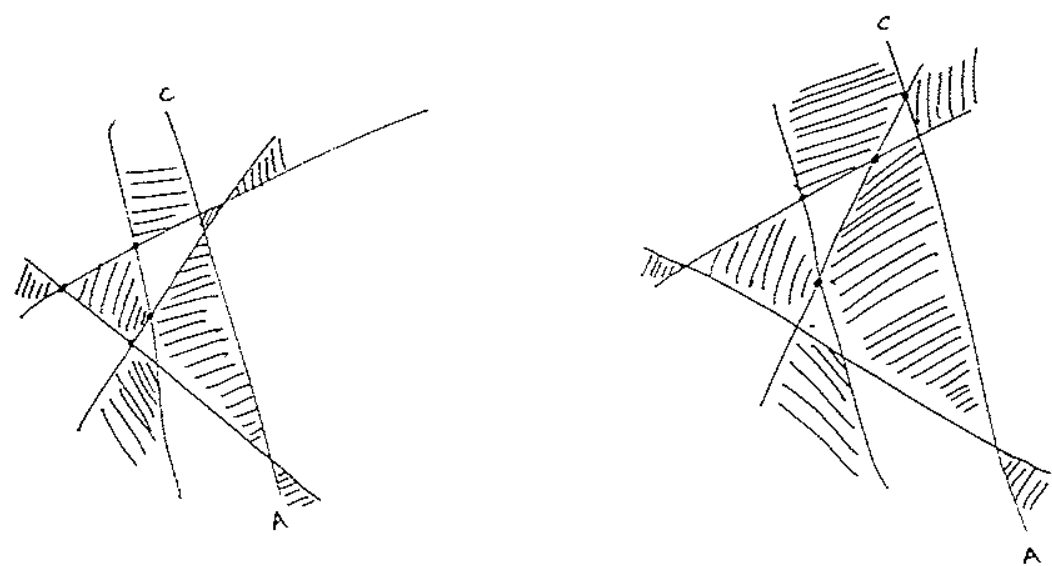

Fig. 39. 

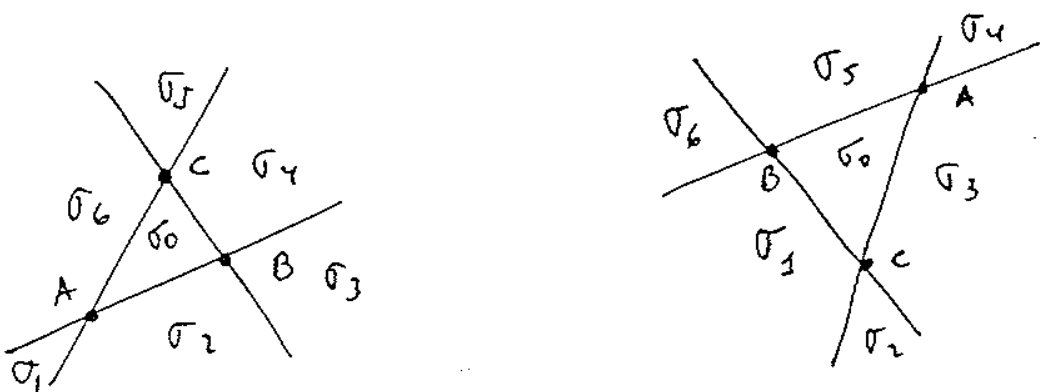

Fig. 40.

$$
\begin{aligned}
W_{1}= & 2 \exp \left(K_{1}^{\prime} \sigma_{6} \sigma_{2}+K_{2}^{\prime} \sigma_{2} \sigma_{4}+K_{3}^{\prime} \sigma_{4} \sigma_{6}\right) \\
& \times \cos h\left(K_{1}^{\prime} \sigma_{1}+K_{2} \sigma_{3}+K_{3} \sigma_{5}+K^{n}{ }_{1} \sigma_{1} \sigma_{6} \sigma_{2}\right. \\
& \left.+K^{n}{ }_{2} \sigma_{3} \sigma_{2} \sigma_{4}+K^{n}{ }_{3} \sigma_{5} \sigma_{4} \sigma_{6}\right) \\
W_{2}= & 2 \exp \left(K_{1}^{\prime} \sigma_{3} \sigma_{5}+K_{2}^{\prime} \sigma_{5} \sigma_{1}+K_{3}^{\prime} \sigma_{1} \sigma_{3}\right) \\
& \times \cos h\left(K_{1} \sigma_{4}+K_{2} \sigma_{6}+K_{3} \sigma_{2}+K^{n}{ }_{1} \sigma_{4} \sigma_{3} \sigma_{5}\right. \\
& \left.\quad+K^{n}{ }_{2} \sigma_{6} \sigma_{5} \sigma_{1}+K^{n}{ }_{3} \sigma_{2} \sigma_{1} \sigma_{3}\right)
\end{aligned}
$$

where the summation on $\sigma_{0}$ has been performed. The invariance of the partition function under the shift of lines requires that $W_{1}$ must equal $W_{2}$. This requirement is very close to the Yang-Baxter equation, in fact it is the Yang-Baxter equation when we identify the Boltzmann weights of the vertex model with the components of a factorized $S$-matrix (see Eq. (8.14) and Fig. 38). The condition:

$$
W_{1}=W_{2}
$$

becomes to six equations:

$$
\begin{aligned}
& \exp \left(2 K_{j}^{\prime}+2 K_{k}^{\prime}\right)=\frac{\cos h\left(K_{1}+K_{2}+K_{3}+K^{n}{ }_{i}-K^{n}{ }_{j}-K^{n}{ }_{k}\right)}{\cosh \left(-K_{i}+K_{j}+K_{k}-K^{n}{ }_{i}+K^{n}{ }_{j}+K^{n}{ }_{k}\right)}(8.23) \\
& \exp \left(2 K_{j}^{\prime}-2 K_{k}^{\prime}\right)=\frac{\cosh \left(K_{i}-K_{j}+K_{k}-K^{n}{ }_{i}+K^{n}{ }_{j}+K^{n}{ }_{k}\right)}{\cosh \left(K_{i}+K_{j}-K_{k}-K^{n}{ }_{i}+K^{n}{ }_{j}+K^{n}{ }_{k}\right)}
\end{aligned}
$$

where $(i, j, k)$ is any permutation of $(1,2,3)$. A solution of $(8.23)$ and $(8.24)$ is:

$$
K^{n}{ }_{1}=K^{n}{ }_{2}=K^{n}{ }_{3}=K^{n}
$$

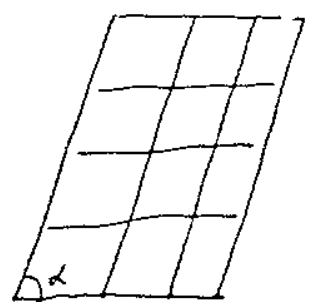

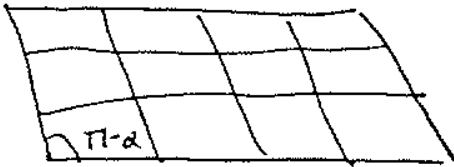

Fig. 41

and

$$
\Delta_{1}=\Delta_{2}=\Delta_{3}=\Delta
$$

where

$$
\Delta=-\sin h 2 K_{i} \sin h 2 K_{i}^{\prime}-\tan h 2 K^{\prime \prime} \cos h 2 K_{i} \cos h 2 K_{i}^{\prime} .
$$

The condition of integrability of a model defined on a generic lattice $\mathcal{L}$ is that any triangle of $\mathcal{L}$.must satisfy Eq. (8.22). This condition is known as $Z$-invariance.. Therefore for an integrable model the interaction coefficient $K^{\infty}$ must be the same for any site triangle. Once we fix the values of $\Delta$ and $K^{n}$ the possible values of the interaction coefficients are determined by (8.27). It is possible to prove that the solutions of (8.27) for $\Delta$ and $K^{n}$ fixed can be parametrized in terms of the angles at the sites. In general a nonregular lattice is integrable if the Boltzmann weights depend only on the angles of the lattice and the system is $Z$-invariant. For the eight-vertex model we have in addition to $Z$-invariance, invariance under crossing. In fact if we represent the Boltzmann weights in terms of a factorized $S$-matrix this one will depend, for a nonregular $Z$-invariant lattice, only on the angles at the sites. Crossing symmetry means that:

$$
S_{i j, k l}(\alpha)=S_{i k, j l}(\pi-\alpha) .
$$

This symmetry is true for a generic $Z$-invariant Baxter model. It is important to point out that crossing symmetry is a consequence of $Z$-invariance and the special form of the Boltzmann weights (see Eq. (8.1)) we have used. This symmetry is very close to modular invariance in the continum limit. In particular it means that the partition functions of the two lattices in Fig. 41 must be the same. Moreover if we take the thermodynamic limit, mantaining the ratio between the number of rows and columns constant we will get in the continum limit, a modular invariant partition function. 


\section{RSOS-models 65}

We begin by defining a RSOS model associated to a Coxeter diagram of type $A^{35}$ :

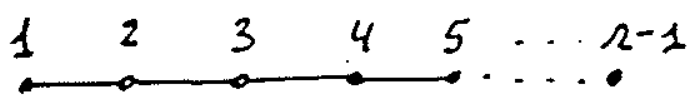

The model is parametrized by the number $r$, the Coxeter number of the diagram (8.28). On a regular lattice the model is defined by associating heights $l_{i}$ to each site with range:

$$
1 \leq l_{i} \leq r-1
$$

and such that

$$
\left|l_{i}-l_{j}\right|=1
$$

for adjacent sites. Notice that the Coxeter diagram (8.28) contains this imformations. For each plaquette we define the Boltzmann weight $W\left(l_{1} l_{2} l_{3} l_{4}\right)$ that must satisfy symmetry under reflections:

$$
W\left(l_{1} l_{2} l_{3} l_{4}\right)=W\left(l_{3} l_{2} l_{1} l_{4}\right)=W\left(l_{1} l_{4} l_{3} l_{2}\right)
$$

The partition function of the model is given by:

$$
Z=\sum_{\text {cong }} \prod W\left(l^{\prime} s\right)
$$

To solve the model we proceed in the same way as with the vertex models. First we represent the partition function in terms of the transfer matrix:

$$
Z=\operatorname{Tr}\left(T^{N}\right)
$$

and then look for the conditions under which the transfer matrix can be diagonalized. The transfer matrix is defined by:

$$
T_{\bar{e}, e^{\prime}}=\prod\left\{W\left(l_{1} l_{2} l_{2}^{\prime} l_{1}^{\prime}\right) W\left(l_{2} l_{3} l_{3}^{\prime} l^{\prime} l_{2}\right) \ldots \ldots\right\}
$$

where $l=\left(l_{1} \ldots l_{m}\right)$ (see Fig. 42). Using different Boltzmann weights, let us say $w^{\prime}$, we obtain

$$
T_{l l^{\prime}}^{\prime}=\prod\left\{w^{\prime}\left(l_{1} l_{2} l_{2}^{\prime} l_{1}^{\prime}\right) \ldots\right\}
$$

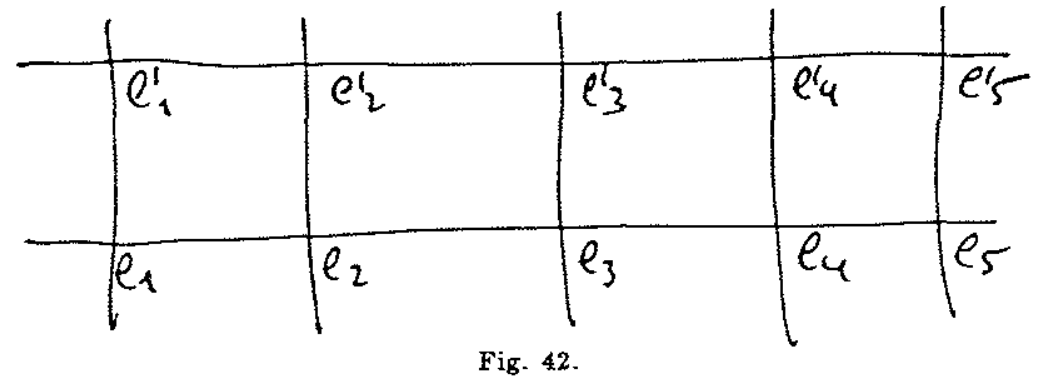

The condition of integrability is:

$$
\left(T T^{\prime}\right)_{\Pi^{\prime}}=\left(T T^{\prime}\right)_{m^{\prime}}
$$

Using techniques similar to these in the previous case, we can write (8.36) as a triangular equation for three different Boltzmann weights $w, w^{\prime}$ and $w^{n}$ :

$$
\sum_{g} w(a b g f) w^{\prime}(f g d e) w^{\prime}(g b c d)=\sum_{g} w^{\prime \prime}(g c d e) w^{\prime}(a b c g) w(f a g e)
$$

where $a, b \ldots$ represent the heights $l_{i}$. Again we can write (8.37) as an equation for a one-parameter family of Boltzmann weights:

$$
\begin{aligned}
& \sum_{g} w(a b g f \mid u) w(f g d e \mid u+v) w(g b c d \mid v) \\
= & \sum_{g} w(g c d e \mid v) w(a b c g \mid u+v) w(f a g e \mid u)
\end{aligned}
$$

The simplest example of SOS-model is the case $r=4$ which corresponds to the Ising model. In this case the heights are 1,2,3 and any configuration can be separated into two pieces: one even and one odd, each living in a different sublattice (Fig. 43). If we change variables to $\sigma_{i}=l_{i}-2$ and we consider the dual lattice we get the standard Ising model on the white sublattice where we color the lattice as with the Baxter models (Fig. 44). On the white sublattice we have plaqnette variable $\sigma_{i}= \pm 1$. Now we can use the Boltzmann weights of the RSOS model to define the interaction coefficients. Using the same notation as that for the Baxter models (see Fig. 36 and 46 ) we arrive at:

$$
\exp \left(K_{i} \sigma_{p} \sigma_{l}+K_{i}^{\prime} \sigma_{q} \sigma_{m}+K^{\prime}{ }_{i} \sigma_{p} \sigma_{q} \sigma_{l} \sigma_{m}\right)=W\left(l_{p} l_{q} l_{l} l_{m}\right)
$$




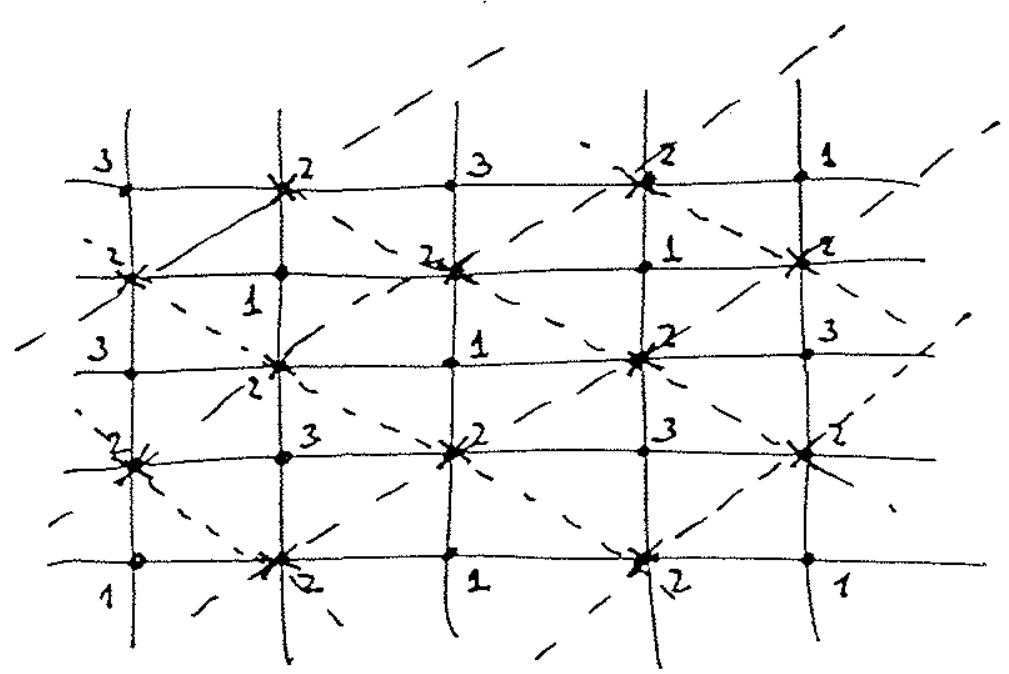

Fig. 43.

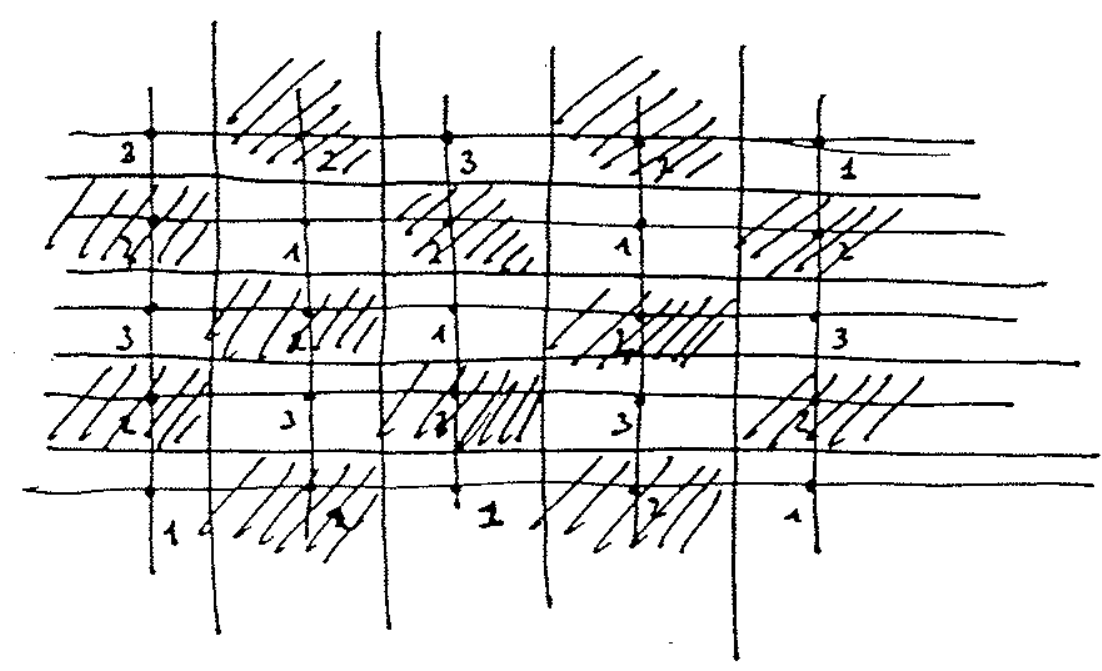

Fig. 44.

For the $r=4$ example $K^{\prime \prime}=0$ and two interaction coefficients, one vertical and another horizontal, are defined by:

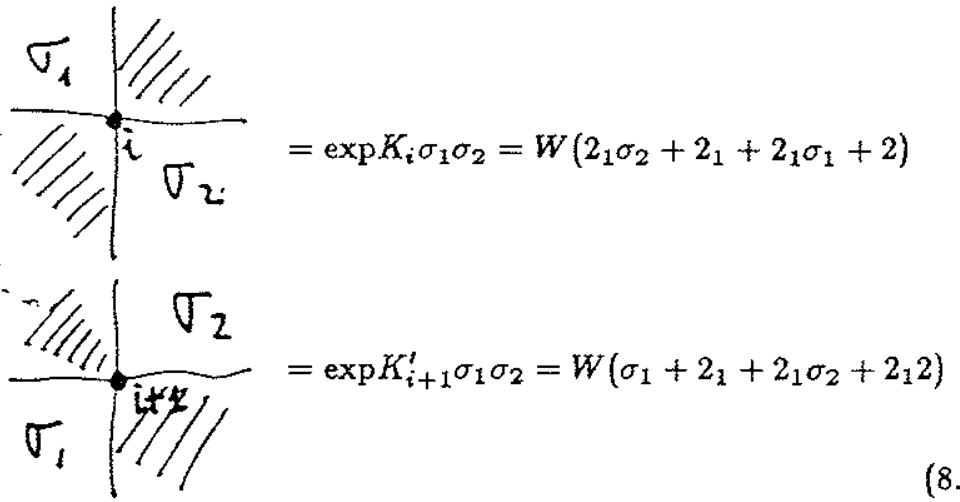

Therefore we see that the RSOS model for $r=4$ is a vertex model with $K^{\prime}=0$. In general if we include spin variables in the black sublattice and we still impose $K^{n}=0$ we will get two Ising models. Let us now consider the Yang-Baxter equation (8.38) for the special case $r=4$. Now:

$$
\begin{aligned}
& \sum_{g} W(a 2 a g 2 \mid u) W(2 g 2 e \mid u+v) W(g 2 c 2 \mid v) \\
& \quad=W(2 c 2 e|v| W(a 2 c 2 \mid u+v) W(2 a 2 e \mid u) .
\end{aligned}
$$

Changing to $\sigma_{i}$ variables (8.41) become precisely the equations determining the $Z$-invariance of the Ising model (Fig. 47). Using reflection symmetry (8.31) we obtain four independent Boltzmann weights:

$$
\begin{array}{ll}
W_{1}(u)=W(2,1,2,3 \mid u) & W_{3}(u)=W(1,2,3,2 \mid u) \\
W_{2}(u)=W(2,1,2,1 \mid u) & W_{3}(u)=W(1,2,1,2 \mid u)
\end{array}
$$

The solution to $(8.41)$ is:

$$
\begin{array}{ll}
W_{1}(u)=\frac{\theta_{1}(u+\pi / 4, p)}{\theta_{1}(\pi / 4, p)} & W_{2}(u)=\frac{\theta_{1}(\pi / 4-u, p)}{\theta_{2}(\pi / 4, p)} \\
W_{3}(u)=\frac{\theta_{1}(u, p)}{\theta_{1}(\pi / 2, p)} & W_{4}(u)=\frac{\theta_{1}\left(\frac{\pi}{2}-u, p\right)}{\theta_{1}\left(\frac{\pi}{2}, p\right)}
\end{array}
$$

where $\theta_{1}(u, p)$ is the odd elliptic theta function. Sinilar arguments apply to other models. 


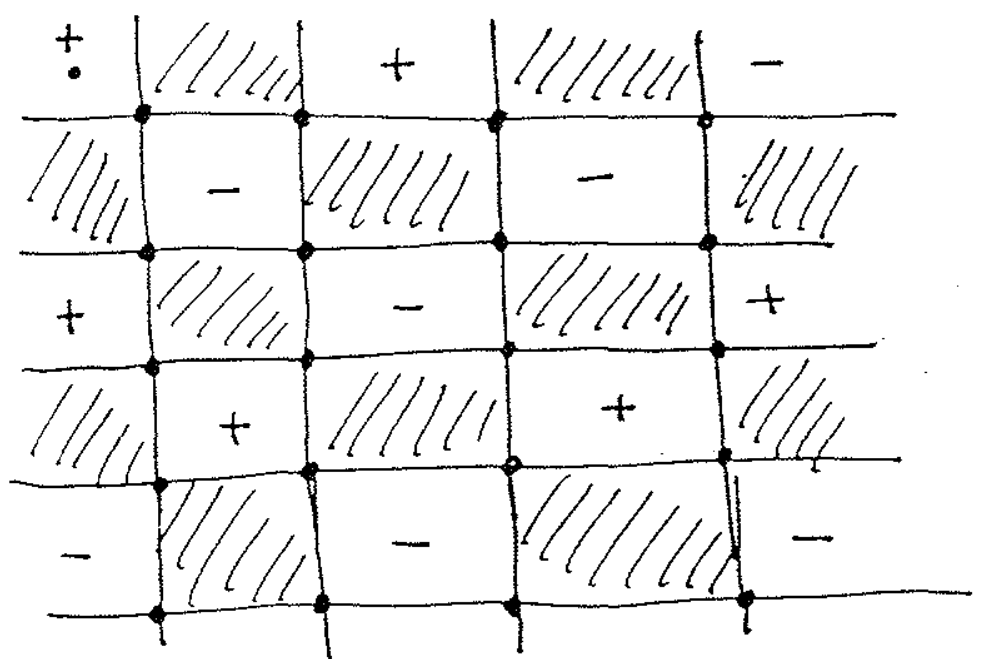

Fig. 45 .

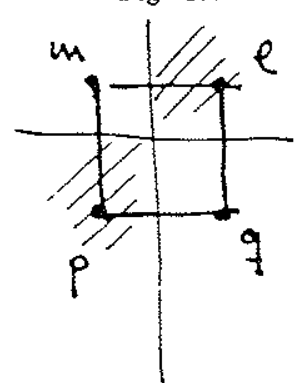

Fig. 46.

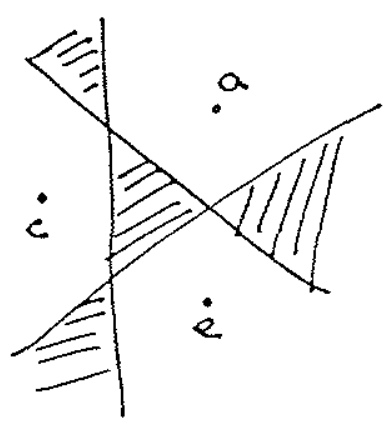

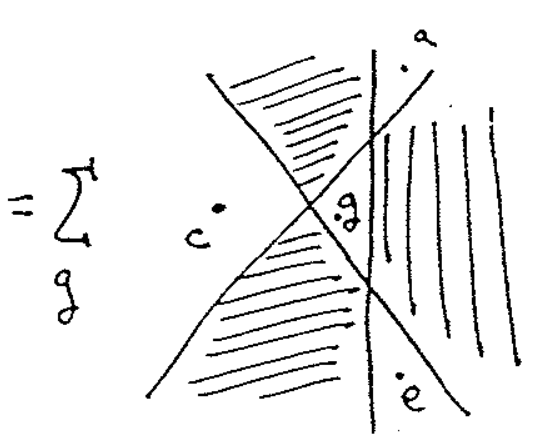

Fig. 47 .

\subsection{Conformal field theory}

It is well-known that conformal field theories can be interpreted as a description of the continuum limit of statistical models of the critical point. In particular the minimal models of the $c<1$ series are in oneto-one correspondence with the critical behavior of restricted SOS models defined on Coxeter diagram of type $A_{r}$. A possible way of relating conformal field theory with lattice systems is through quantum groups, namely by identifying the centralizes of the quantum group with some limit, in the spectral parameter, of the Temperley-Lieb algebra of the lattice system.

At the self-dual point (criticality) the partition function of $r$-SOS models can be written as:

$$
Z(u)=\operatorname{Tr}(V(u) W(u))^{N}
$$

where

$$
\begin{aligned}
& V(u)=\left(1+f(u) e_{1}\right)\left(1+f(u) e_{3}\right) \ldots \\
& W(u)=\left(1+f(u) e_{2}\right)\left(1+f(u) e_{4}\right) \ldots
\end{aligned}
$$

with $u$, the spectral parameter and $e_{i}$ matrices satisfying:

$$
\begin{array}{ll}
e_{i} e_{j} & =e_{j} e_{i} \quad|j-i| \geq 2 \\
e_{i} e_{i \pm 1} e_{i} & =\tau e_{i} \\
e_{i}^{2} & =e_{\nu}
\end{array}
$$

$\tau$ depending on the spenfic model. The matrices $e_{i}$ are defined in terms of Boltzmann weights. More precisely they define a particular representation of the algebra (8.97) on the space of states of the lattice system. The action is defined as follows: (see Fig. 48)

$$
e_{i}\left|l_{1} \ldots l_{i-1} l_{i} l_{i+1} \ldots l_{m}\right\rangle=\sum_{l_{i}} \sum_{l_{i} l_{i+1}}^{l_{i-1} l_{i}^{\prime}} \delta\left(l_{i-1}, l_{i+1}\right) \cdot\left|l_{1} \ldots l_{i-1} l_{i}^{\prime} l_{i+1} \ldots l_{m}\right\rangle
$$

Equation (8.48) is the analog of the one defining the action of the centralizer of the quantum group on the space of conformal blocks. There we know that the representation is given by the quantum $6 j$-symbols. Following this formal analog it is natural to look for a more precise relation between the Boltzmann weights of the lattice model and $q-6 j$-symbols. This relation 


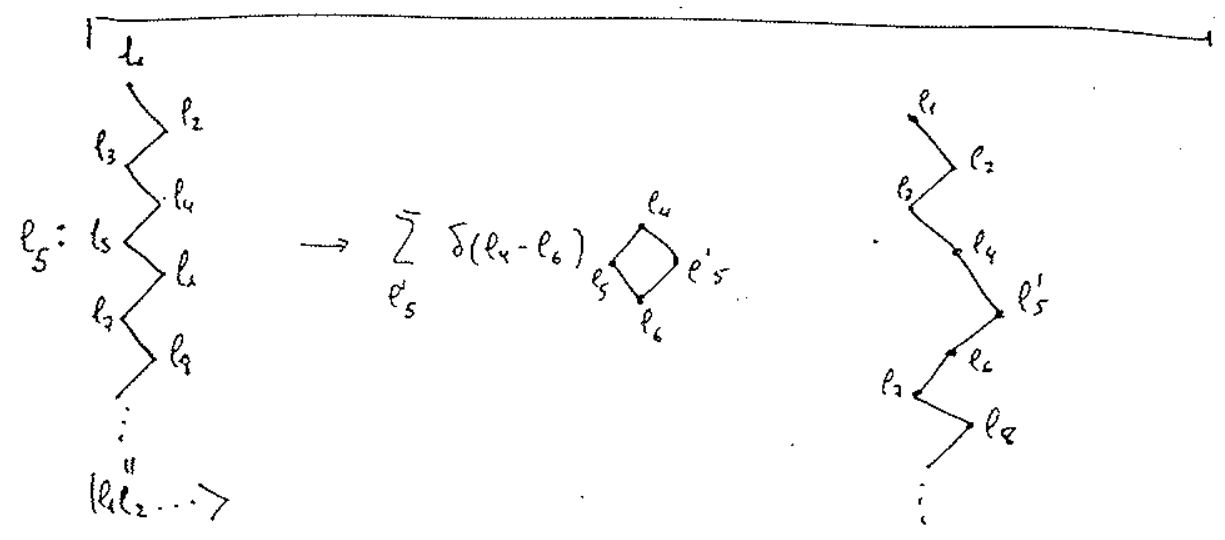

Fig. 48. Graphc representation of the action of an element of the TL algebra.

actually exist (see Ref. 60 for details), for the $u \rightarrow \infty$ limit of $\frac{1}{f(u)} W(\ldots \mid u)$. The reason we need to divide by $f(u)$ is in order to make the representation of the algebra unitary. The same function was used by Zamolodchikov to get from the Boltzmann weights unitary factorized $S$-matrices. The reason for the $u \rightarrow \infty$ limit is less clear from a physical point of view. The other natural limit $u \rightarrow 0$ will give us the identity matrices. A qualitative argument for motivating the $u \rightarrow \infty$ limit is interpreting this limit as the one corresponding to working with the lattice model on a degenerate torus with modula paramter $\tau=1$. This is a natural quantum group limit at the level of characters. In fact quantum characters can be thought of as a regularized version of the Kac-Moody characters in the limit $\tau=1$. These considerations are by no means rigorous but they provide some intuition about the physical content of the $u \rightarrow \infty$ limit.

\section{Knots Conformal Field Theories and Statistical Mechanics 9.1 Knot theory}

Knot theory studies the embeddings of $S^{1}$ in arbitrary 3-manifolds. ${ }^{37,38}$ Until recently the theory was well-establish for embedding in $S^{3}$. During the last year important progress has been achieved with the work of Witten to extend the theory to arbitrary three manifolds using Topological gauge theories. In physics knot theory is related to CFT, statistical mechanics and the theory of Integrable Systems.

The simplest way to build a knot is to start with a $\phi^{4}$-diagram (the knot universe) and replace each vertex by a monplanar crossing (Fig. 49). With the rules in Fig. 49 we can associate to an $n$-vertex graph $2^{n}$ knots. This of course contains an enormous overcounting. A more algebraic way of constructing a knot is by closing braids. Fig. 50. In this way we can write knots in terms of words in the braid group whose classes generate them (the knot in Fig. 50 comes from closing $\sigma_{1} \sigma_{2}^{-1}$, and iterating this operating $\left(\sigma_{1} \sigma_{2}^{-1}\right)^{n}$ produces a girl's braid). This procedure is once again very redundant. From a mathematical point of view we are only interested in topologically equivalent classes of knots. Two knots are equivalent if we can transform one into the other by a continuous deformation. A theorem in knot theory guarantees that two equivalent knots can be transformed into each other via Reidemeister moves. There are three types of moves shown in Fig. 51:

In particular applying a type I move we see that for any element $g \in B_{n}$ the knots $\hat{g}$ and $\hat{g} \sigma_{n+1}$ (the carat indicates the closure of the braid) are topologically equivalent (Fig. 52).

After the knots have divided into equivalence classes according to the Reidemeister moves we would like to characterize each class by some topological invariants. As with characteristic classes we can define these invariants axiomatically, and these axioms define the link polynomials. We now describe three link polynomials. The simplest one is the AlexanderConway polynomial given by the axioms:

(A1). To each knot $K$ there is associated a one-variable polynomial $P_{k}(t)$ with integer coefficients such that if $K$ and $K^{\prime}$ are equivalent they have the same polynomials.

(A2). The polynomial is normalized by requiring that for the unknot (unknotted circle) $P_{s^{1}}(t)=1$.

(A3). Alexander Conway skein rule: Given three knots $K^{+}, K^{-}, L$ which only differ in one crossing as:
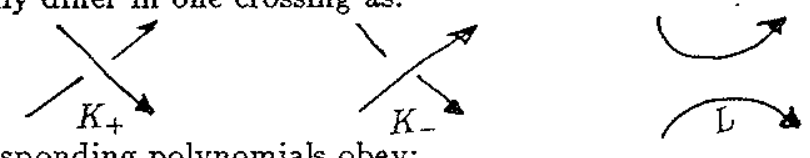

the corresponding polynomials obey:

$$
P_{K^{+}}(t)-P_{K^{-}}(t)=t P_{L}(t) .
$$

$K_{+}$is an overcrossing. $K_{-}$an undercrossing and $L$ is the splicing of the crossing. By applying the skein move (9.1) a sufficient number of times we eventually transform any knot or link into collection of unknotted circles. 


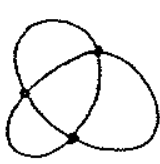

$+$
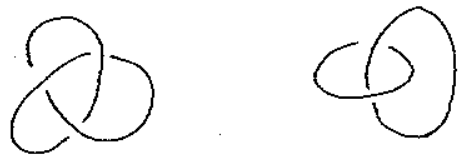

Fig. 49. Rules to build knots from $\phi^{4}$-graphs.
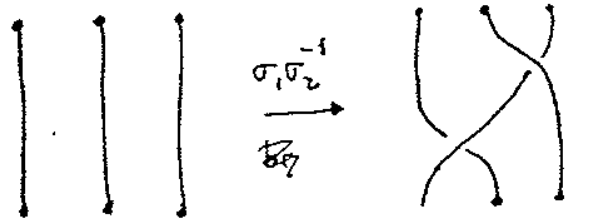

close

$\rightarrow$

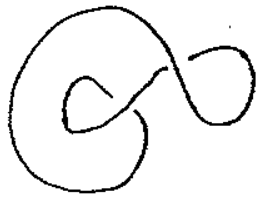

Fig. 50. Knot generated by closing braids.
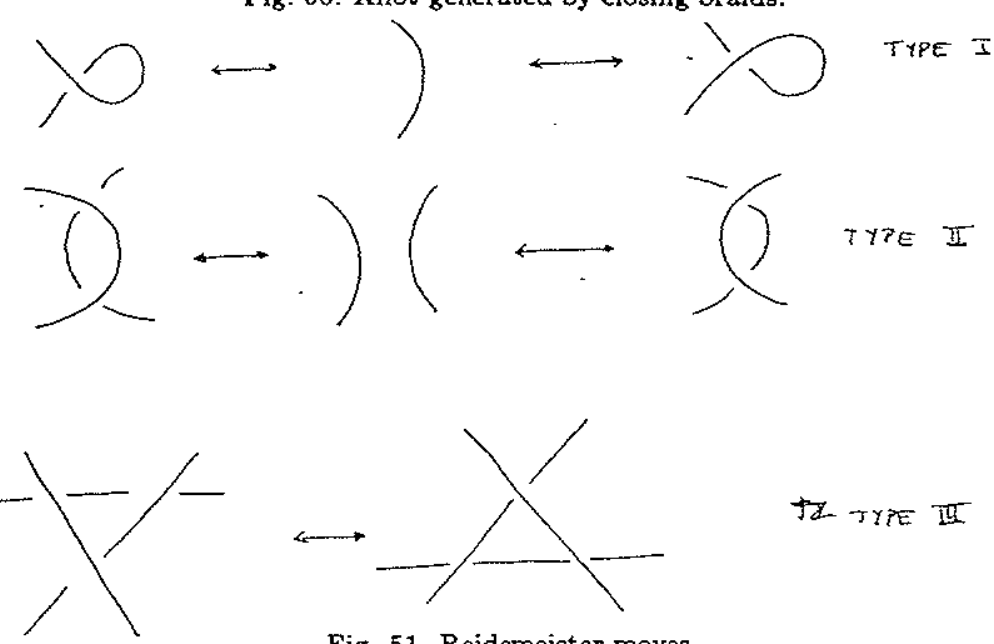

TZ. TYFE II

Fig. 51. Reidemeister moves.

Notice however that many inequivalent knots may have the same polynomial. For instance, the Alexander-Conway polynomial is unable to distinguish between a knot and its mirror image even when they are topologically distinct. Progress in the construction of knot invariants can be measured by how precisely the knot is characterized by the invariant. Some simple computations of the Alexander-Conway polynomial $P_{K}(t)$ following easily
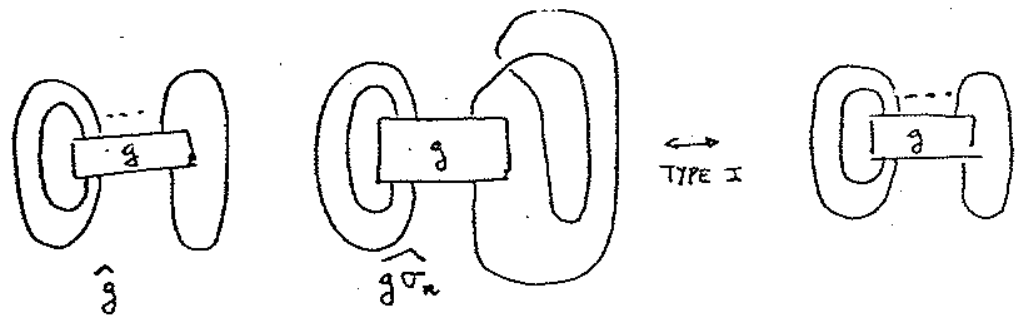

Fig. 52. Equivalence of $\hat{g}$ and $\hat{g \sigma_{n}}$.

from the skein relation are:

$P(O)=1 \quad P(\mathrm{O} . .3)=0$

$P(L)=0$ (if $L$ splits into several pieces)

$P(\theta-\theta)=P(\theta 0)=P(100)$

for a link:

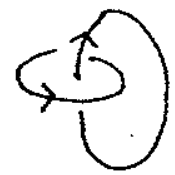

$\mathrm{K}+$

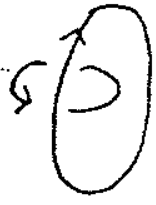

K -

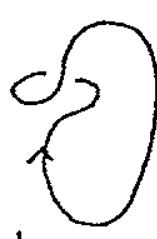

Since $P_{K^{-}}=0(9.3),(9.2)$ imply $P_{K+}(t)=t$. Next for the trefoil:

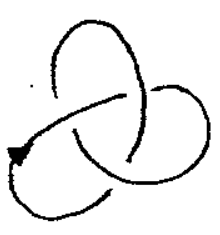

then

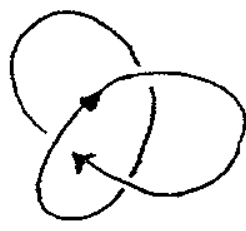

$$
P_{T}(t)=1+t^{2}
$$

the link with $2 n$-crossings

$$
p\left(S_{\vdots}^{5}\right)=n t
$$


The skein axiom is more difficult to motivate than the other two. It is very specific to knots in $S^{3}$. In the skein moves we always consider the crossing as contained in some ball $B_{3}$ and this is certaily always the case in $S^{3}$. After a finite number of skein moves any knot is transformed into a collection of unknotted unlinked circles. As a final example of how the skein relation works, we show in Fig. 53 how to prove that the split link has a vanishing polynomial.

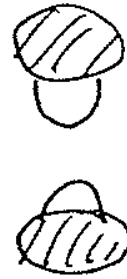
$=$

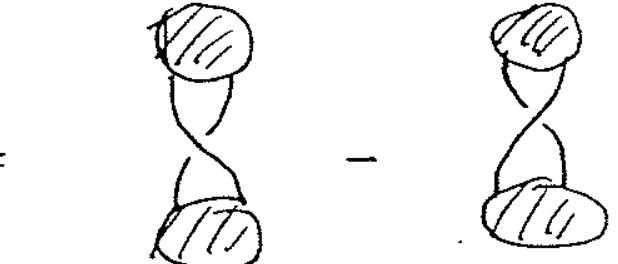

Fig. 53. The alexander-Conway polynomial vanishes for a split link.

Before studying generalizations of $P_{K}(t)$, it is instructive to consider the skein relation from the point of view of the braid group characterization of knots. The knots $K^{+}, K^{-}, L_{0}$ are related by

$$
\begin{aligned}
& K^{+}=\sigma_{1}^{2} K^{-} \\
& L=\sigma_{1} K^{-}
\end{aligned}
$$

This is illustrated in Fig. 54.

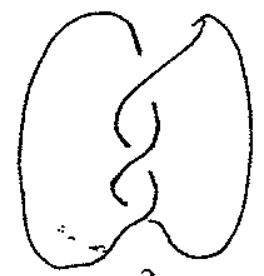

$\sigma_{i}$

$k^{+}$

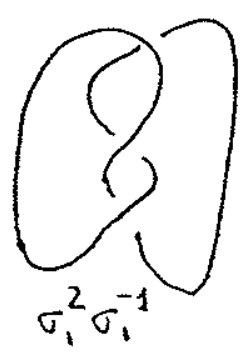

$k^{-}$

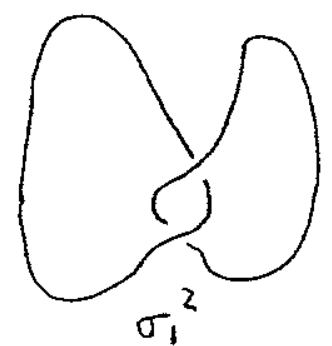

Lo
Fig. 54. Example of equation (9.6)

Equation (9.6) is very appealing from the point of view of the previous sections. If we work with Hecke algebras instead of braid groups (9.6) will give us a particular type of skein rule. From the defining relation of a Hecke algebra of type $A_{n}$ :

$$
\sigma_{1}^{2}=(q-1) \sigma_{i}+q
$$

and:

$$
K^{+}-q K^{-}=(q-1) L
$$

very close to the skein rule in (A3). This indicates that the skein rule is related to the Hecke representations of the braid group.

We now consider a two-variable generalization of the previous polynomial ${ }^{66}$ In some convenient normalizations the new polynomial is defined by the skein rule

$$
\begin{gathered}
\left(\lambda^{1 / 2} q^{1 / 2}\right)^{-1} P_{K^{+}}(q, \lambda)-\left(\lambda^{1 / 2} q^{1 / 2}\right) P_{K-}(q, \lambda) \\
=\left(q^{1 / 2}-q^{-1 / 2}\right) P_{L}(q, \lambda) .
\end{gathered}
$$

A particular case of $(9.8)$ is the Jones polynomial ${ }^{41}$ defined by

$$
V_{K}(q)=P_{K}(q, q)
$$

To motivate the new polynomials it is convenient to have a more algebraic representation of the Reidemeister moves. They correspond to the following two transformations on the braid representation (the second one appears graphically in Fig. 55)

$$
\begin{array}{lcl}
\text { MARKOV I } & \alpha \cdot \beta \rightarrow \beta \cdot \alpha & \alpha, \beta \in B_{n} \\
\text { MARKOV II } & \alpha \cdot \sigma_{n}^{ \pm 1} \rightarrow \alpha & \alpha \in B_{n} .
\end{array}
$$

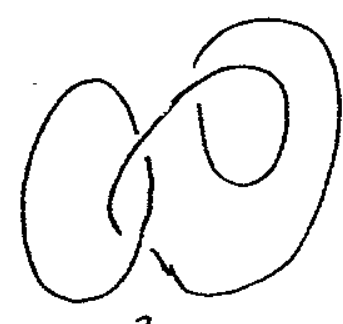

$\sigma_{1}^{2} \sigma_{2}$

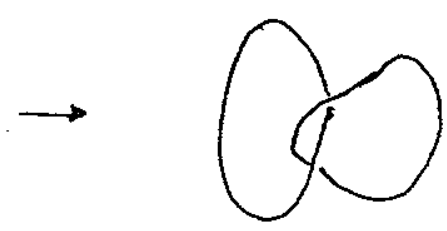

${ }^{2}$
Fig. 55. Markor move of type II. 
The transformations (9.10) on braid groups are known as Markov transformations. In general any invariant polynomial must be defined by some functional on $\bigcup_{n=0}^{\infty} B_{n}$ invariant under the two Markov moves (9.10). Such an invariant will automatically satisfy axiom 1 . To have a constructive way of defining such a functional, we need to characterize how this functional changes under the set of transformations which unknot any close braid. For knots in $S^{3}$ we can use skein moves in the unknotting procedure. The skein move on the other hand requires a Hecke representation of the braid group, $g_{i}^{2}=(q-1) g_{i}+q$. Thus to define invariant polynomials of links in $S^{3}$ (and with a skein relation as above) we need:

(i). A Hecke representation $H_{n}(g)$ of $B_{n}$.

(ii). A functional defined on $H_{\infty}=\cup H_{n}(q)$ invariant under the Markov moves (9.10) for the particular embedding defined in (1).

There is a trace on $H_{\infty}$ which will allow us to construct a knot invariant. This is the Ocneanu trace defined by,

(1). $\operatorname{tr}(a b)=\operatorname{tr}(b a)$

(2). $\operatorname{tr}(1)=1$

(3). $\operatorname{tr}\left(x g_{n}\right)=z \operatorname{tr} x, x \in H_{n}, g_{n} \in H_{n+1}$ the complex parameter $z$ characterizes the trace. We now use the Ocneanu trace to derive the polynomials (9.8) and (9.9). First we need the representation $B_{n} \rightarrow H_{n}(q)$. Following Jones ${ }^{41}$ we define:

$$
\begin{aligned}
\pi_{\lambda}: B_{n} & \rightarrow H_{n}(q) \\
& \sigma_{i} \rightarrow \pi_{\lambda}\left(\sigma_{i}\right)=\sqrt{\lambda} g_{i}, \lambda=\frac{1-q+z}{q z}
\end{aligned}
$$

(the elements $g_{i}$ are the $H_{n}(q)$ generators). It is now easy to verify that:

$$
P_{\hat{\alpha}}(q, \lambda) \equiv\left(-\frac{1-\lambda q}{\sqrt{\lambda}(1-q)}\right)^{n-1}(\sqrt{\lambda})^{e} \operatorname{tr} \pi_{\lambda}(\alpha)
$$

$\alpha \in B_{n}$ and $\hat{\alpha}$ is the knot obtained closing $\alpha$ which is invariant under (9.10). The term $(\sqrt{\lambda})^{e}$ is there to normalize the polynomial so that $P_{O}(q, \lambda)=1$ and the Hecke algebra relation implies a skein rule. The trace in (9.12) satisfies the rules (1)-(3) for the value of $z$ appearing on the representation (9.11). Using the Hecke algebra relation:

$$
(\sqrt{\lambda} \sqrt{q})^{-1} P_{K^{+}}(q, \lambda)-(\sqrt{q} \sqrt{\lambda}) P_{K^{-}}(q, \lambda)=\left(q^{1 / 2}-q^{-1 / 2}\right) P_{L}(q, \lambda)
$$

and Jones polynomial is a particular case of this one $(\lambda=q)$. In CFT it is the Jones polynomial which appears more naturally and we would like to explain it in more detail. The first significant difference between the Jones polynomial and (9.8) is that one uses the Temperley-Lieb algebra instead of the Hecke algebra. This algebra was discovered in physics in the study of critical phenomena of some solvable models. We know from Sec. 6 that this algebra appears as the centralizer of $\mathrm{SU}(2, q)$ for the fundamental representation. Mathematically this algebra is given by a set of projections $e_{1}, \ldots, e_{n}$ satisfying:

$$
\begin{array}{lll}
e_{i} e_{j} & =e_{j} e_{i} \quad|i-j| \geq 2 \\
e_{i}^{2} & =e_{i} \\
e_{i} e_{i \pm 1} e_{i} & =\tau e_{i}
\end{array}
$$

for some $\tau$ which defines the algebra. Out of (9.13) we can construct a Hecke algebra representation:

$$
\begin{aligned}
& \tau^{-1}=2+q+q^{-1} \\
& g_{i}=q e_{i}\left(1-e_{i}\right) .
\end{aligned}
$$

It is straightforward to verify that $g_{i}$ satisfies that Hecke relations together with:

$$
g_{i} g_{i+1} g_{i}-g_{i} g_{i+1}-g_{i+1} g_{i}+g_{i}+g_{i+1}-1=0
$$

This constraint explains the relation with $\mathrm{SU}(2, q)$, it means that the three row antisymmetrizer vanishes automatically (see Secs. 6 and 7). The restriction from $(9.8)$ to the Jones polynomial (9.9) corresponds to the representation of $B_{n}$ into the algebra (9.13).

$$
\phi\left(\sigma_{i}\right)=q e_{i}-\left(1-e_{i}\right)
$$

Using the skein rule (9.8) it is easy to compute the polynomial $P(q, \lambda)$ for the trefoil knot:

$$
P_{\text {trefoil }}(q, \lambda)=\frac{1}{\lambda}+\frac{1}{\lambda q^{2}}+\frac{1}{\lambda q}+\frac{1}{\lambda^{2} q^{2}}
$$

(the mirror image corresponds to changing $\sqrt{\lambda q}$ to $-(\sqrt{\lambda q})^{-1}$ and obviously (9.17) changes. The Jones polynomial, or its two variable extension has succeeded in distinguishing some knots from their mirror images, thus going beyond the Alexander-Conway polynomial). At the end on this section we will give a derivation of these invariants from RCFT using the fusion rules and the duality properties. 


\subsection{State models:Turaev and Kauffman invariants}

Given a Yang-Baxter matrix $R: V \otimes V \rightarrow V \otimes V$, we can define a representation of the braid group $B_{n}$ into the space Aut $\left(V^{\otimes n}\right)$. This was done before in our study of centralizers for quantum groups. Recall that

$$
\pi\left(\sigma_{i}\right)=R_{i}=1 \otimes \ldots \otimes R_{i, i+1} \otimes \ldots \otimes 1: V^{\otimes n} \rightarrow V^{\otimes n}
$$

and from the Yang-Baxter equation the $R_{i}$ provides a representation of $B_{n}$. Using this representation we will define link polynomials. We will obtain a function on $\operatorname{Aut}\left(V^{\otimes n}\right)$ invariant under Markov moves. Following Turaev ${ }^{39}$ (see also Ref. 67) we define an extended Yang-Baxter system characterized by a Yang-Baxter matrix $R$ and an isomorphism $\mu: V \rightarrow V$ transforming a basis $\left\{\nu_{i}\right\}$ into $\left\{\mu_{i} \nu_{i}\right\}$ satisfying

$$
\begin{aligned}
\left(\mu_{i} \mu_{j}-\mu_{k} \mu_{l}\right) R_{i j}^{k l} & =0 \\
\sum_{j} R_{i j}^{k j} \mu_{j} & =a b \delta_{i}^{k} \\
\sum_{j}\left(R^{-1}\right)_{i j}^{k j} \mu_{j} & =a^{-1} b \delta_{i}^{k}
\end{aligned}
$$

for some $a, b$. From these condition it follows that

$$
T(\alpha)=a^{-w(\alpha)} b^{-n} \operatorname{tr}\left(\pi(\alpha) \mu^{\otimes n}\right)
$$

is invariant under Markov moves. Here $\varepsilon$ is the wraith number for the word $\alpha \varepsilon B_{n}$, defined by $w\left(\sigma_{i}\right)=-w\left(\sigma_{i}^{-1}\right)=1, w(\alpha \beta)=w(\alpha)+w(\beta) .(\pi(\alpha)$ is the representation (9.18)). Then:

$$
\begin{array}{ll}
T(\alpha \beta)=T(\beta \alpha) & \alpha \beta \in B_{n} \\
T\left(\alpha \sigma_{n}^{ \pm 1}\right)=T(\alpha) & \alpha \in B_{n} .
\end{array}
$$

The interest of this invariant is that it can be automatically defined in the context of quantum groups where the $R_{i}$ are the generators of the centralizer of the group in $V^{\otimes r}$. As explained in Sec. 7 for quantum groups there is always one element $u$ in the algebra satisfying (9.19) where $b$ is the quantum define different polynomials.

In order to visualize the polynomial defined in (9.20) it is useful to have its skein rule representation. An interesting theorem of Turaev states that for any extended Yang-Baxter system there is always a skein rule of the form:

$$
\sum_{i=-p}^{+p} a: t^{i} T\left(\alpha_{i}\right)=0
$$

where the knots $\alpha_{i}$ appear in Fig. 56 and the coefficients $a_{i}$ depend on the extended system. The skein relation (9.22) once again indicates that the Turaev invariants defined so far are naturally related to knots in $S^{3}$.

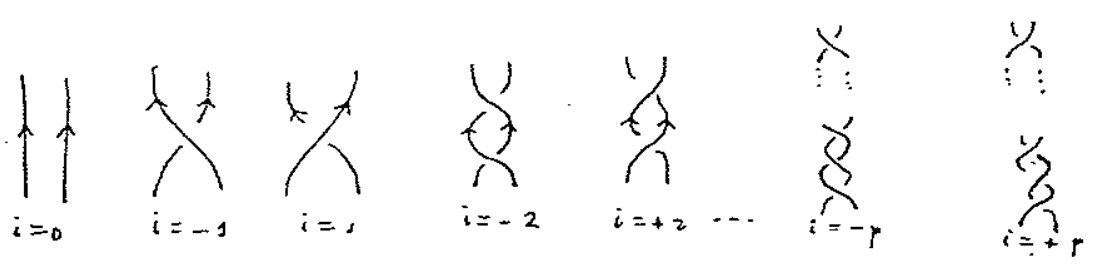

Fig. 56. Crossing entering in the skein rule (9.22).

The invariant (9.20) can be expressed more explicitly in the language of quantum groups. As an example take $G=\mathrm{SU}(2, q) V=V_{1 / 2}$ the fundamental representation. Then $\pi(\alpha)$ is defined in terms of $R^{1 / 21 / 2}(6.32)$ and we represent the blocks in (6.4) $f_{j}^{1 / 21 / 2 \ldots 1 / 2} \equiv W_{j}=$ space of blocks with $N \phi_{1 / 2}$ fields and one spectator field with spin $j$, where the representation of $\alpha \in B_{n}$ in $W_{j}$ is defined in terms of the $6 j$-symbols;:

$$
\begin{aligned}
& \rho^{j}(x)\left(\frac{\ddot{j}_{2}^{v_{2}}||^{1 / 2}}{y_{2} a_{1} \cdots a_{N-2}} d\right) \otimes e_{m}^{j} \\
& =\sum_{m^{\prime} s}\left\{\begin{array}{lll}
1 / 2 & 1 / 2 & a_{1} \\
m_{1} & m_{2} & m
\end{array}\right\}_{q} \ldots\left\{\begin{array}{lll}
a_{N-2} & 1 / 2 & j \\
m_{N-2} & m_{N} & j
\end{array}\right\} \\
& \times R(\alpha)\left[e_{m_{1}}^{1 / 2} \times \ldots \times e_{m_{N}}^{1 / 2}\right]
\end{aligned}
$$

where $e_{m}^{j}$ is a basis of $V^{j}$ and $R(\alpha)$ is the representation of the word $\alpha \in B_{n}$ using $R^{1 / 21 / 2}$. The invariant (9.20) now becomes

$$
T(\alpha)=\sum_{j} w_{j} \operatorname{tr} \rho^{j}(\alpha) .
$$


In the next subsection we will explain detail how to compute the $w_{j}$ coefficients starting from a more general point of view. This will tie rather nicely different approaches to the computation of knot and link invariants (RCFT, quantum groups, Chern-Simons actions etc.)

\subsection{Rational conformal field theories and knots invariants}

The aim of this section is to show that using the basic imgredients of a RCFT, namely the fusion matrices $N_{i j}^{k}$, the modular matrix $S_{i j}$ and the braid matrices $B_{p p^{\prime}}\left[\begin{array}{ll}j & k \\ i & l\end{array}\right]$, one can construct invariants of links. ${ }^{25}$

Our construction will follow the lines of Jone's original approach. ${ }^{40}$

Let us start with a RCFT by considering the conformal blocks $F_{\phi, i}^{(n)}$ in Fig. 26 where $\phi$ is a given primary field which appears $n$ times in the block, and the index $i$ runs over all possible primary fields of the theory.

The number of conformal blocks of the type $\mathcal{F}_{\phi, i}^{(n)}$ is given in terms of the fusion matrix $N_{\phi j}^{k}$ by:

$$
d_{\phi, i}^{(n)}=\sum_{i_{1}, \ldots, p_{n-2}} N_{\phi p_{1}}^{i} N_{\phi p_{2}}^{p_{1}} \ldots N_{\phi \phi}^{p_{n-2}}=\left(N_{\phi}^{n-1}\right)_{\phi}^{i}
$$

where

$$
\left(N_{\phi}\right)_{i}^{j} \equiv N_{\phi i}^{j} .
$$

From this equation one finds the recursion formula

$$
\begin{aligned}
& d_{\phi, i}^{n+1}=\sum_{j} d_{\phi, j}^{n}\left(N_{\phi}\right)_{j}^{i} \\
& d_{\phi, i}^{2}=N_{\phi \phi}^{i}
\end{aligned}
$$

where both $N_{\phi i}^{j}$ and $d_{\phi, i}^{n}$ are non-negative integers. It is convenient to organize the integers $d_{\phi, i}^{n}$ into a Bratteli diagram. The $n^{\text {th }}$ row of a Bratteli diagram is given by the set of numbers $d_{\phi, i}^{n+1}$ belonging to the $(n+1)^{\text {th }}$ row and the number $d_{\phi, j}^{n}$ of the $n^{\text {th }}$ row we draw one arrow whenever $N_{\phi j}^{i}=1$, two arrows whenever $N_{\phi j}^{i}=2$, etc. This is represented in Fig. 57 .

The arrows in a Bratteli diagram indicates the way the dimensions of the conformal blocks with $n$-external $\phi$ fields sum up to reproduce the corresponding conformal blocks with $(n+1)$ extend $\phi$ fields. Conversely the different paths in the Bratteli tree from the top to a given element $j$ in the $n$th row gives the different internal states in the block $\mathcal{F}_{\phi, j}^{(n)}$.

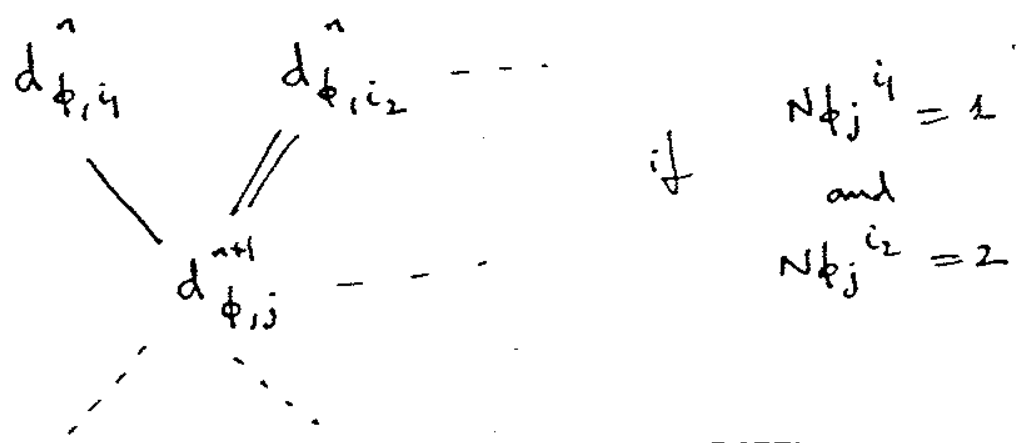

Fig. 57. Pattern for Bratteli diagrams in RCFT's
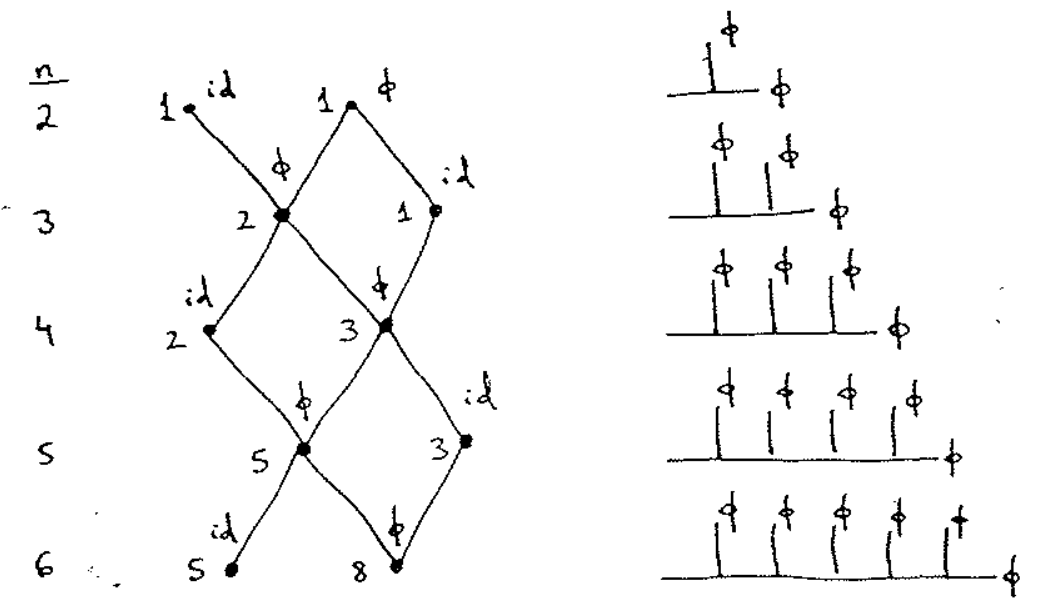

Fig. 58 .

As a simple example consider a RCFT with only two primary fields: $i d$ and $\phi$, and fusion rules:

$$
\begin{aligned}
& i d \times i d=i d \\
& i d \times \phi=\phi \\
& \phi \times \phi=i d+\phi
\end{aligned}
$$

which yield the following fusion matrices

$$
N_{i d}=\left(\begin{array}{ll}
1 & 0 \\
0 & 1
\end{array}\right) \quad N_{\phi}=\left(\begin{array}{ll}
0 & 1 \\
1 & 1
\end{array}\right)
$$

The Bratteli diagram in this case is a Fibonacci tree, and the associated conformed block are as in Fig. 58. 
Let us now return to the conformal blocks $\xi_{\phi, i}^{(n)}$. The duality properties of RCFT's imply that a braid operation on the $\phi$ legs leaving the spectator field fxed mixes the internal channels in $\xi_{\phi i}^{(n)}$.

If $g \in B_{n}$ one obtains in this way a representation of $B_{n}$ on $\mathcal{F}_{\phi, i}^{(n)}$, of dimension $d_{\phi, i}^{n}$. Explicitly:

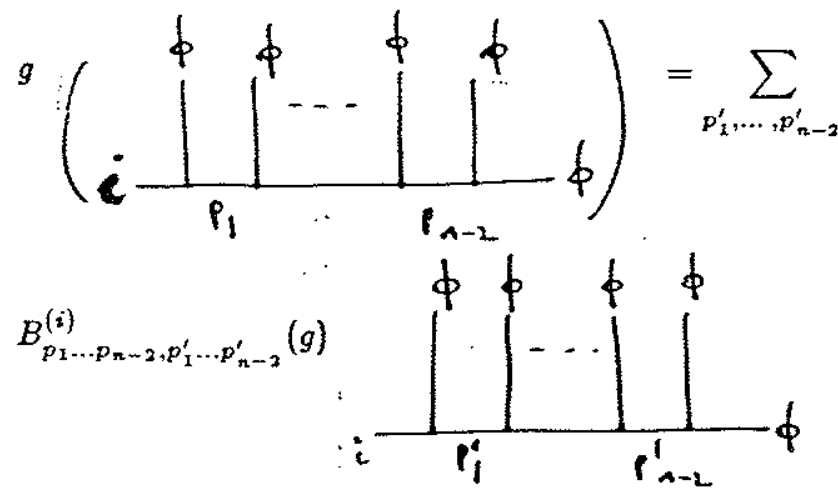

where $B^{(i)}(g)$ is the braid matrix associated to the element $g \in B_{n}$ and has dimension $d_{\phi, i}^{n}$.

In fact since $B_{n}$ is generated by the transpositions $\sigma_{1}, \ldots, \sigma_{n-1}$ it is enough to know the following braid coefficients (Fig. 4 and 8 ):
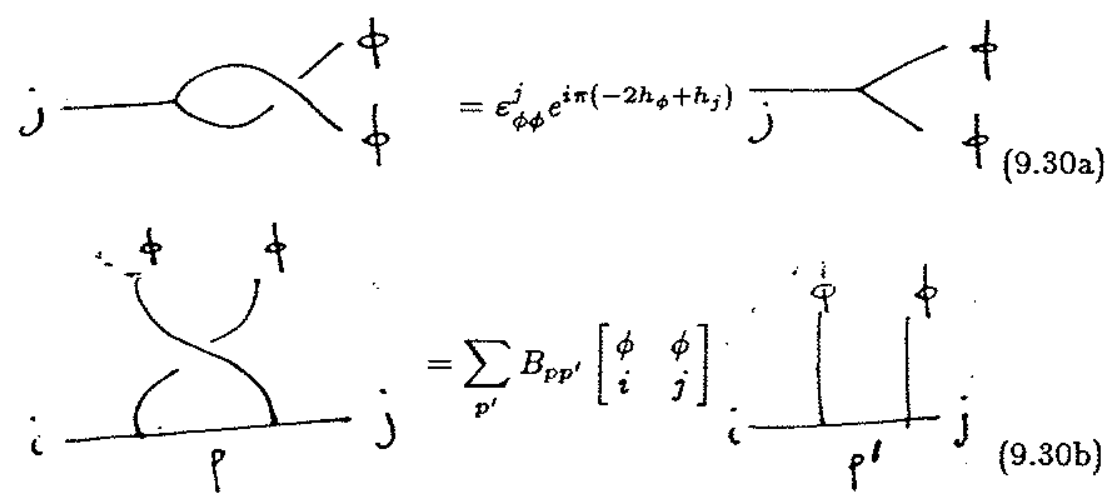

The whole set of braiding matrices $B^{(i)}(g)$ appearing in Eq. (9.29), for a fixed expectator belongs to the space $M_{d_{d, i}^{n}}(\mathbb{C})$ of complex square matrices of dimension $d_{\phi, i}^{n}$. Taking into account all possible expectators leads us to which is a finite-dimensional $C^{*}$-algebra.

In fact what we obtain is an infinite sequence of $C^{*}$-algebras $\left\{A_{\phi}^{n}\right\}_{n=2,3, \ldots}$ whit an inclusion relation:

$$
A_{\phi}^{2} \subset A_{\phi}^{3} \subset \ldots \subset A_{\phi}^{n} \subset A_{\phi}^{n+1} \subset \ldots
$$

given by (9.26). In other words the inclusion matrix for each step in (9.32) is the fusion matrix $N_{\phi}$ of the RCFT.

. We have thus arrived at the situation presented by Jones in Ref. 40 .

More generally, we have two finite matrix algebra $A, B$ and $B \subset A$. Then

$$
\begin{aligned}
& A=\oplus_{i} M_{n_{i}}(\mathbb{C}) \\
& B=\oplus_{\alpha} M_{m_{\alpha}}(\mathbb{C})
\end{aligned}
$$

and the inclusion is completely specified by the inclusion matrix $\Lambda_{i \alpha}$ whose entries are non-negative integers relating the dimensions of the irreducible representations of $A, B$

$$
n_{i}=\sum_{\alpha} \Lambda_{i \alpha} m_{\alpha}
$$

The inclusion matrix for the sequence $(9.32)$ of our RCFT is $\left(N_{\phi}\right)_{i}^{j}$.

In Jones construction once we have the inclusion $B \subset A$ then one can generate an infinite sequence as in (9.32):

$$
B \subset A \subset A \otimes_{B} A \subset A \otimes_{B} A \otimes_{B} A \subset \ldots
$$

where $A \otimes_{B} A$ is defined by the condition $x b \otimes y=x \otimes b y(b \in B ; x, y \in A)$. If $\Lambda$ is the inclusion matrix for $B \subset A$, then $\wedge^{T}$ is the inclusion matrix for $A \subset A \otimes_{B} A$, and so on.

The $\Lambda$ matrix for the Jones algebra $A_{\beta, n}$ described in Sec. 7 with $\beta=4 \cos ^{2} \pi / m$ can be constructed as the incidence matrix of a partition of the Dinkin diagram of $A_{m-1}$ into two disjoint subsets, such that no pair in the same subset are adjacent in $A_{m-1}$.

If $m$ is odd then we have the Dinkin diagram

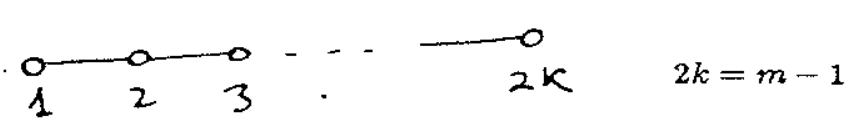


and the associated incidence matrix

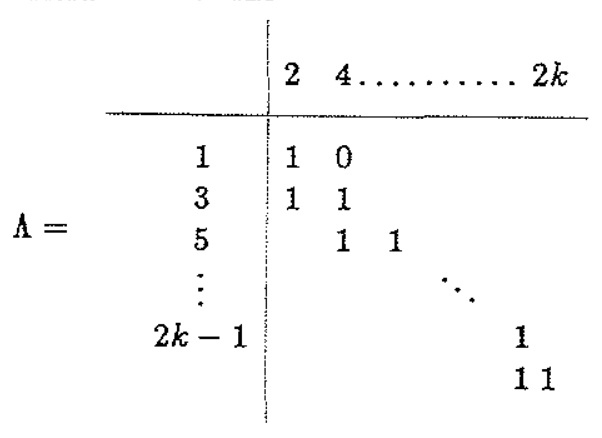

Notice $\Lambda \in M_{\frac{m-1}{3}}(\mathrm{~N})$.

If $m$ is even the Dinkin diagram is

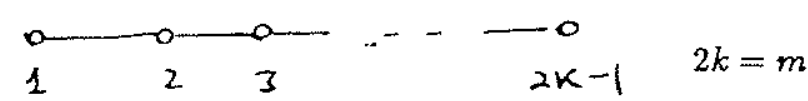

yielding:

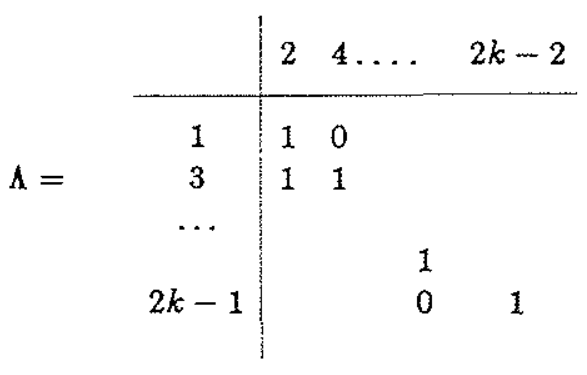

$\Lambda \in M_{\frac{m}{3}, \frac{m-3}{3}}(\mathbb{N})$.

It can be shown that the largest eigenvalue of the matrix $\wedge^{T} \wedge$ is $\beta=4 \cos ^{2} \pi / m$. Notice that $m$ is the Coxeter number of $A_{m-1}$, and in fact the same result is true for incidence matrices built up from the Dinkin diagrams of $D_{m}(m \geq 4), E_{6}, E_{7}$ and $E_{8}$.

Comparing the sequences (9.32) and (9.35) one may wonder about the relations between them. A complete understanding of this point is not yet available, but its elucidation will shed same light in the classification of fusion rules of RCFT. We shall limit ourselves here to exhibit the connection between (9.32) and (9.35) in some particular cases.

In order to interpret the inclusion matrices (9:36) and (9.37) as the fusion matrices of any RCFT we shall permute the Column labels. Let us take for simplicity the case where $m$ is odd so that $\Lambda$ is a square matrix.
The permuted incidence matrix which we shall call $\wedge_{\phi}$ is

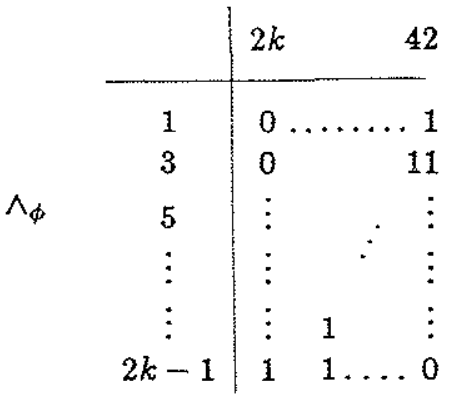

Notice that $\wedge_{\phi}^{T}=\wedge_{\phi}$ and that the highest eigenvalue of $\left(\wedge_{\phi}\right)^{2}$ is given by $\beta=4 \cos ^{2} \pi / m$. Now we can look for RCFT's with $\wedge_{\phi}$ as the fusion matrix of same field $\phi$. We have already encountered one case given by Eq. (9.27) and (9.28). The matrix $N_{\phi}$ of (9.28) is identical to $\wedge_{\phi}$ for $m=S$. The case $m=7$ would yield a fusion matrix:

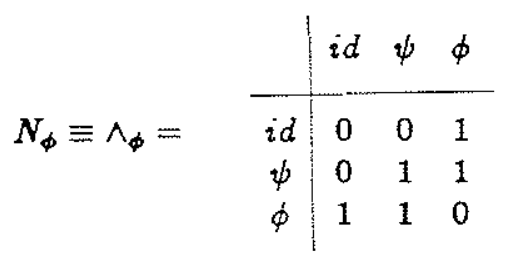

which requires the existence of 3 primary fields $i d, \phi$ and $\psi$. An example of (9.39) is provided by the minimal series with $c=-\frac{68}{7}$. Notice that the fusion matrix $N_{\phi}$ can be derived from theat of $N_{\phi}$ :

$$
\phi \times \phi=i d+\psi+N_{\psi}=N_{\phi}^{2}-1=\left(\begin{array}{lll}
0 & 1 & 0 \\
1 & 1 & 1 \\
0 & 1 & 1
\end{array}\right)
$$

Sometimes the fusion matrices of a RCFT are related to incidence matrices in a more complicated way. Take for instance the $\mathrm{SU}(2)_{k} \mathrm{WZW}$ model for $k$ odd. The fusion matrix $N_{1 / 2}$ can be derived from the fusion rules:

$$
\begin{aligned}
& \phi_{1 / 2} \times \phi_{0}=\phi_{1 / 2} \\
& \phi_{1 / 2} \times \phi_{j}=\phi_{j-1 / 2}+\phi_{j+1 / 2} \quad j<k / 2 \\
& \phi_{\frac{1}{2}} \times \phi_{\frac{k}{2}}=\phi_{\frac{k}{2}-1}
\end{aligned}
$$


then:

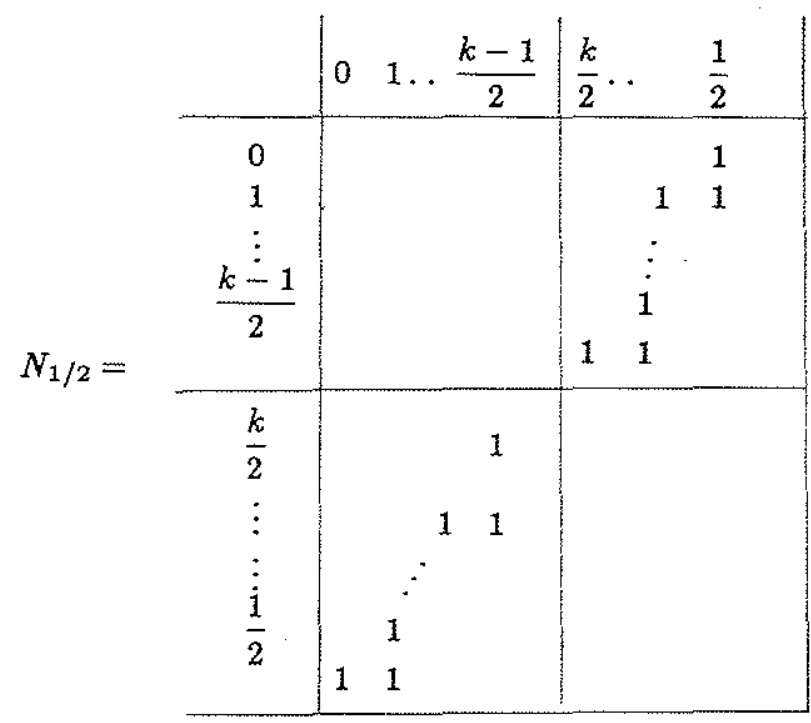

Hence:

$$
N_{1 / 2}=\left(\begin{array}{cc}
0 & \wedge_{1 / 2} \\
\wedge_{1 / 2} & 0
\end{array}\right)
$$

where $\wedge_{1 / 2}$ is the incidence matrix (9.38) for $m=k+2$. (The block structure form of $N_{1 / 2}$ display the graded structure of the representation of $\left.\mathrm{SU}(2)_{k}\right)$.

Notice that in the cases we have considered the largest eigenvalue of $\wedge^{T} \wedge$ is equal to $\left(S_{\phi 0} / S_{00}\right)^{2}$, for example for $\mathrm{SU}(2)_{k}$.

$$
\beta^{1 / 2}=2 \cos \frac{\phi}{k+2}=\frac{S_{1 / 20}}{S_{00}}=[2]_{q} \quad q=e^{2 \pi i / k+2}
$$

We see once again the ubiquity of the q-numbers. One should perhaps remark that not all the fusion matrices of a given RCFT have such a nice description as incidence matrices of Dinkin diagram. That seems to be a property of the fusion rules of certain primary fields in the theory which play somehow the rule of the fundamental representation for the corresponding RCFT.

After all the previous examples we want a trace on the sequence of algebras $A_{\phi}^{n}$ with the Markov properties.

Since each factor in (9.33) has the ordinary trace for square matrices we define a "global" trace for $A$ and $B$ as:

$$
\begin{aligned}
& \operatorname{tr}^{A}(x)=\sum_{i} t_{i}^{A} \operatorname{tr}\left(x_{i}\right) \\
& \operatorname{tr}^{B}(y)=\sum_{\alpha} t_{\alpha}^{B} \operatorname{tr}\left(y_{\alpha}\right)
\end{aligned}
$$

where $x \in A, x_{i} \in M_{n_{i}}(\mathbb{C})$, similarly for $B$.

The condition that $\mathrm{tr}^{A}$ descends to $\mathrm{tr}^{B}$ through the inclusion (9.34) implies the constraint

$$
\sum_{i} t_{i}^{A} \wedge_{i \alpha}=t_{\alpha}^{B}
$$

which is dual to (9.34). This time however $t^{A}$ and $t^{B}$ are not forced to the integers. If $t_{i}^{A}$ and $t_{\alpha}^{B}$ are positive numbers we shall say that $\operatorname{tr}^{A}$ and $t r^{B}$ are positive traces.

In our case we want to construct traces on $A_{\phi}^{n}$ with the Markov properties. Following (9.44) we define

$$
\operatorname{tr}^{(n)}(x)=\sum t_{\phi, i}^{n} \operatorname{tr}\left(x_{i}\right)
$$

where $x \in A_{\phi}^{n}$ and $x_{i} \in M_{d_{\phi, i}^{n}}$. Then $(9.45)$ reads

$$
\sum_{j}\left(N_{\phi}\right)_{i}^{j} t_{\phi ; j}^{n+1}=t_{\phi, i}^{n}
$$

Using the Verlinde theorem we can find a solution to (9.47):

$$
t_{\phi, j}^{n}=\frac{S_{j 0}}{S_{00}}\left(\frac{S_{00}}{S_{\phi 0}}\right)^{n} .
$$

Since $\frac{S_{j 0}}{S_{00}} \geq 0$, we obtain a positive trace on the whole family $A_{\phi}=\oplus_{n} A_{\phi}^{n}$.

From the previous discussion we have shown that for each primary field $\phi$ we can define the maps.

$$
\begin{aligned}
& B_{n} \stackrel{\mathrm{RCFT}}{\longrightarrow} A_{\phi}^{n \stackrel{\mathrm{RCFT}}{\longrightarrow}} \mathbb{C} \\
& g \stackrel{\longrightarrow}{\longrightarrow}\left\{B_{p_{1} \ldots p_{n-3}, p_{1}^{\prime} \ldots p_{n-2}^{\prime}}^{(i)}\right\} \longrightarrow \operatorname{tr}_{(g)}^{(n)}
\end{aligned}
$$


where

$$
\operatorname{tr}^{(n)}(g)=\left(\frac{S_{00}}{S_{\phi 0}}\right)^{n} \sum_{j} \sum_{p_{1} \ldots p_{n-2}} \frac{S_{j 0}}{S_{00}} B_{p_{1} \ldots p_{n-2}, p_{1} \ldots p_{n-2}}^{(j)}(g)
$$

This trace obviously satisfies the properties $\operatorname{tr}^{(n)}(\alpha \beta)=\operatorname{tr}^{(n)}(\beta \alpha)$ for $\alpha, \beta \epsilon$ $B_{n}$. As a consequence of Verlinde theorem it also satisfies

$$
\operatorname{tr}^{(n)}(1)=1
$$

Hence to assert that $\mathrm{tr}^{(n)}$ is a Markov trace means that it satisfies:

$$
\operatorname{tr}^{(n+1)}\left(\alpha v_{n}^{ \pm 1}\right)=z_{ \pm} \operatorname{tr}^{(n)}(\alpha) \quad \alpha \in B_{n}
$$

for some $z_{ \pm} \in \mathrm{C}$

For RCFT's where duality properties can be represented in terms of quantum groups this equation is indeed satisfied. If one does not want to rely on quantum groups, we need to invoke a result of Jones which guarantees under very general circumstances that for chains of embeddings of algebras ... $\subset \boldsymbol{A}_{n} \subset \boldsymbol{A}_{n+1} \subset \ldots$ The trace defined here has the Markov property (9.52). Independently from these general arguments we can describe the condition imposed by (9.52) on the braiding matrices.

First of all the value of $z_{ \pm}$is found easily from:

$$
z_{ \pm}=\operatorname{tr}^{(2)} \sigma_{1}^{ \pm 1}=\frac{e^{\mp 2 \pi i h_{\phi}}}{\left(S_{\phi 0} / S_{00}\right)}
$$

Then (9.52) is equivalent to:

$$
\sum_{j} S_{j 0} B_{p p}\left[\begin{array}{ll}
\phi & \phi \\
j & k
\end{array}\right]( \pm)=e^{ \pm 2 \pi i h_{\phi}} S_{p o} N_{\phi k}^{P}
$$

This equation is the analog of the Turaev's conditions (9.19) and implies in particular (choosing $k=0$ ):

$$
\sum_{j} S_{j 0} N_{\phi \phi}^{k} \varepsilon_{\phi \phi}^{j} e^{i \pi h_{j}}=S_{\phi_{0}} e^{4 \pi i h_{\phi}}
$$

It would be interesting to show dierctly that (9.54) follows from the general principle of RCFT. If this is the case then we would have a Markov trace and therfore link and knots invariants/for any RCFT.
Now it is straightforward to construct a polynomial invariant under the Markov moves $g \rightarrow \alpha g \alpha^{-1}$ and $\alpha \rightarrow \alpha \sigma_{n}^{ \pm 1}$ where $\alpha, g \in B_{n}, \sigma_{n} \in B_{n+1}$ : If $g \in B_{n}$, define

$$
T(g)=\left(\frac{S_{\phi 0}}{S_{00}}\right)^{n} e^{2 \pi i h_{\phi} w(g)} \mathrm{tr}^{(n)} g
$$

The coefficient $w(g)$ is the wraith of the braid $g(9.20)$.

It is clear that $T(g)$ satisfies the condition $T\left(\alpha g \alpha^{-1}\right)=T(\alpha)$, and $T$ is also invariant under the Markov move of type II:

$$
\begin{aligned}
T\left(g \sigma_{n}\right) & =\left(\frac{S_{\phi 0}}{S_{00}}\right)^{n+1} e^{2 \pi i h_{\phi} w\left(g \sigma_{n}\right)_{\mathrm{tr}}(n+1)}\left(g \sigma_{n}\right) \\
& =\left(\frac{S_{\phi 0}}{S_{00}}\right)^{n+1} e^{2 \pi i h_{\phi}} e^{-2 \pi i h_{\phi}}\left(\frac{S_{00}}{S_{\phi 0}}\right) \operatorname{tr}^{(n)}(g)=T(g) .
\end{aligned}
$$

Similarly

$$
T\left(g \sigma_{n}^{-1}\right)=T(g)
$$

As an example, consider $n$ unlinked, unknotted circles. $T$ is the case gives

$$
T=\left(\frac{S_{\phi 0}}{S_{00}}\right)^{n}
$$

In RCFT, the dimensions of all primary fields are rational numbers ${ }^{19,20}$ and we can always write the dimensions as $h_{j}=p_{j} / N \bmod$ 1. This implies that:

$$
\begin{aligned}
T\left(\sigma_{1}^{2 N}\right) & =e^{2 \pi i h_{\phi} 2 N} \sum_{j} \frac{S_{j 0}}{S_{00}} N_{\phi \phi}^{j} e^{i \pi 2 N\left(2 h_{\phi}-h_{j}\right)} \\
& =\sum_{j} \frac{S_{j 0}}{S_{00}} N_{\phi \phi}^{j}=\left(\frac{S_{\phi 0}}{S_{00}}\right)^{2}
\end{aligned}
$$

by the properties of $S$. Graphically this is interpreted by saying that the link shown in Fig. 59 is not distinguished in this theory from two unlinked, unknotted circles.

Since all the braid group generators are related to $\sigma_{1}$ by conjugation, this implies that

$$
T\left(\sigma_{i}^{2 N}\right)=\left(\frac{S_{\phi 0}}{S_{00}}\right)^{2}
$$




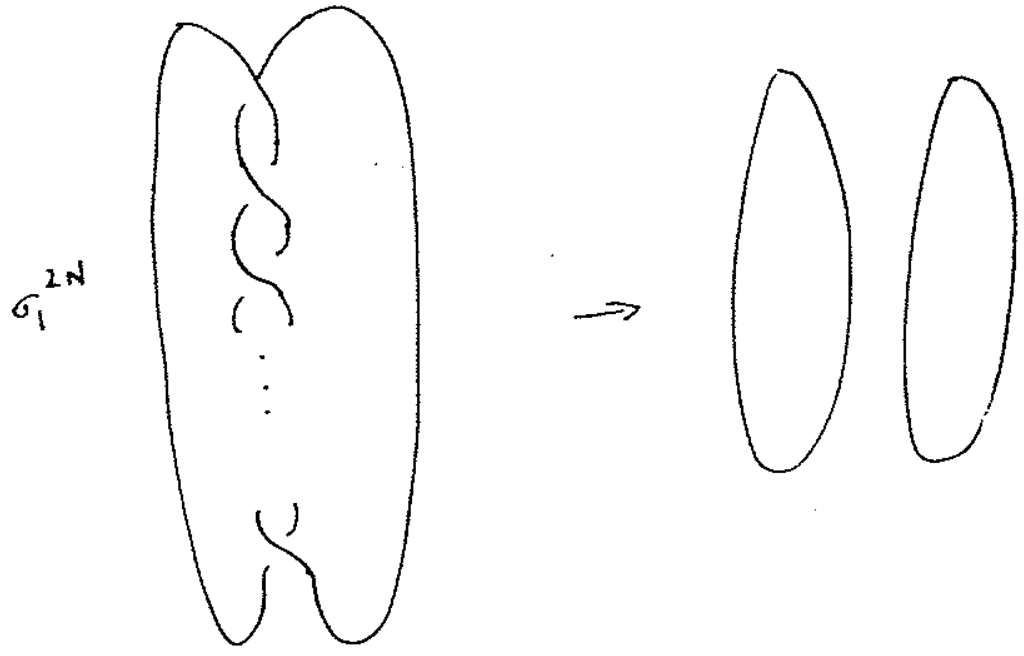

Fig. 59.

gives some constraints on the level of complexity of knots and links that can be distinguished by a given RCFT.

As a final application we consider the $\mathrm{SU}(2)_{k}$ WZW theory. Recalling that $S_{j 0} / S_{00}=[2 j+1]_{q}$ with $q=e^{2 \pi i / k+2}$ we deduce for $\phi=\phi_{j}=1 / 2$ the polynomial:

$$
T(g)=q^{3 / 4 w(g)} \sum_{j}[2 j+1] \operatorname{tr}_{j}(g) \quad g \in B_{n}
$$

where $j$ is constrained to be in the range determined by the fusion rules of $S U(2)_{k}$ in the decomposition of the tensor product $V_{1 / 2}^{\otimes n}$. Similar computations can be carried out for other groups and it gives a conformal field theory derivation of the Ocneanu decompositions in terms of Young tableaux of the Jones polynomials.

\subsection{Knots on arbitrary 3- manifolds}

In this final paragraph we describe some aspects of Witten's construction of knot on arbitrary 3-manifolds. ${ }^{33}$ This extension is based on a 3-dimensional topological gauge theory whose Lagrangian is the threedimensional Chern-Simons invariant. The invariant polynomials are repre- stroke provides an intrinsic geometrical interpretation of knots in arbitrary 3-manifolds.

The metric independent Chern-Simons action is

$$
S[A]=\frac{i k}{4 \pi} \int_{M} \operatorname{tr}\left(A d A+\frac{2}{3} A^{3}\right)
$$

and the first step in Witten's construction is to use (9.58) to construct a modular functor ${ }^{68}$ on the moduli space of Riemann surface with distinguished points. One can think about this construction by extending the operator formalism to three dimensions. In two dimensions the operators formalism associate a ray to any Riemann surfact $\Sigma$ with parametrized boundaries $\partial \Sigma=c_{1} \cup c_{2} \cup \ldots \cup C_{n},\left|\Sigma_{i} C_{j}, \ldots, C_{n}\right\rangle$ (Fig. 60) in the tensor product $\otimes^{n} \psi_{c_{i}}$ of Hilbert spaces associated to each component $c_{i}$ of $\partial \Sigma$. In three dimension $\Sigma$ is replaced by a 3-manifold $M$ and $\partial M=c_{i} \cup \ldots \cup c_{n}$ is a set of 2D surfaces. Hence to $(M, \Sigma)$ with $\Sigma=\partial M$ we associate a state $(M, \Sigma)$ in $\mathcal{K}_{\Sigma}$ (Fig. 61). We have two maps:

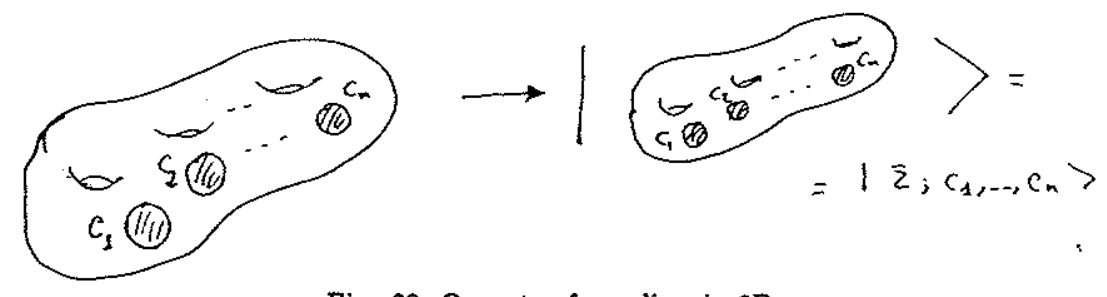

Fig. 60. Operator formalism in $2 \mathrm{D}$.

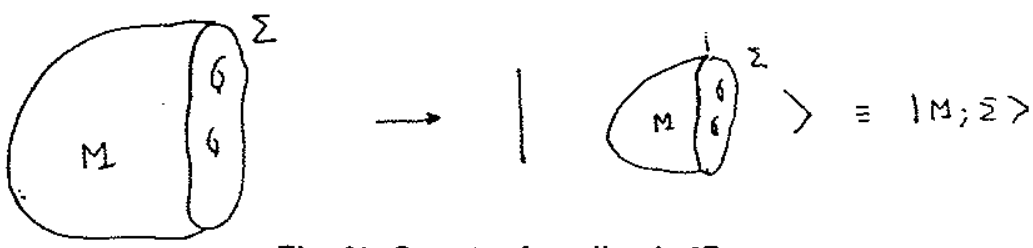

Fig. 61. Operator formalism in 3D.

(i) $\Sigma \rightarrow K_{\Sigma}$

(ii) $(M, \Sigma) \rightarrow|M ; \Sigma\rangle \in \not{\not} \Sigma$

The topological Langrangian (9.58) can be used to define these two maps in a way compatible with surgery manipulations. States in $H_{\Sigma}$, or more generally in $\mathcal{K}_{\left(\Sigma, P_{1}, \ldots, P_{n}\right)}$ (see Fig. 62) do not depend on the geometrical properties of $\Sigma$. The Hilbert space $\mathcal{H}_{\left(\Sigma, P_{1}, \ldots, P_{n}\right)}$ defines a flat vector bundle on the moduli space of Riemann surfaces with punctures. Using 
the Schrodinger representation for the states in $\mathcal{H}_{\left(\Sigma, P_{1}, \ldots, P_{n}\right)}$ the states $\left|M, \Sigma, P_{1}, \ldots, P_{n}\right\rangle$ are functionals on the space of gauge equivalent classes of connections satisfying Gauss' law for external charges at the punctures $P_{1}, \ldots, P_{n}$ :

$$
\frac{k}{8 \pi} \varepsilon^{i j} F_{i j}^{a a}=\sum_{i} \delta\left(X-X_{i}\right) T_{i}^{a}
$$

$$
1
$$

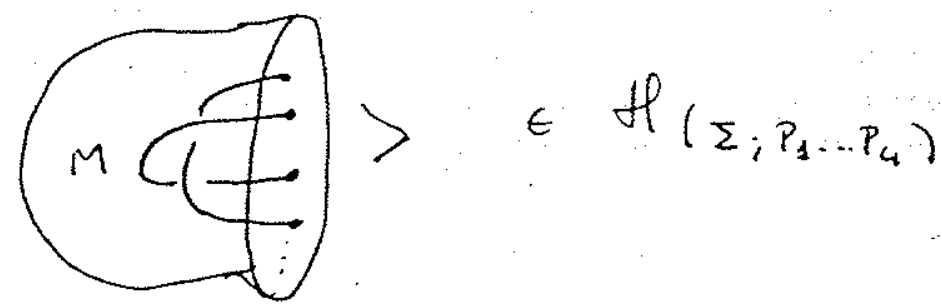

Fig. 62. An element of $K_{\left(\Sigma ; P_{1} \ldots P_{4}\right)}$

The spaces $\mathcal{H}_{\left(\Sigma, P_{1} \ldots P_{n}\right)}$ are representation spaces for the mapping class group of $\Sigma-\left\{P_{1}, \ldots, P_{n}\right\}$. In particular we can braid the points $P_{1}, \ldots, P_{n}$. This operations lifts to a transformation on the fiber of the Hilbert bundle defined by $\mathcal{H}_{\left(\Sigma, P_{1}, \ldots, P_{n}\right)}$ (Fig. 63).

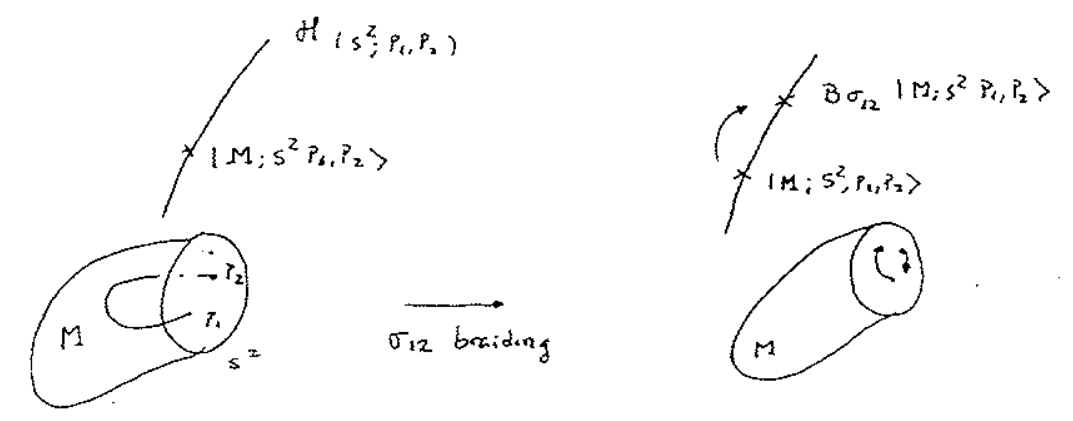

Fig. 63. Lift of the braiding transformation to the Hilbert bundle.

The spaces $\mathcal{K}_{\left(\Sigma, P_{1} \ldots P_{n}\right)}$ when we associate to the punctures representations $R_{i}$ of the gauge group are finite dimensional and they define irreducible representations of the braiding transformations. Moreover these spaces can be identified with the spaces of conformal blocks for a level $k$ WZW model. Here we present heuristic argument for this identification.
First, the bundle $\forall_{\left(\Sigma ; P_{1} \ldots P_{n}\right)}$ is flat over the moduli space of Riemann surfaces with punctures. The same thing happens in conformal field theory where the conformal blocks can be interpreted as a basis of sections of a finite dimensional flat bundle defined on the moduli space. Second let us consider the space $\mathcal{K}_{D}$ where $D$ is a disc. Using the temporal gauge and Gauss'law $\varepsilon^{i j} F_{i j}^{a}=0$ we see that $K_{D}$ is the geometric quantization of $L G / G$, more explicitly the space of sections of a line bundle defined on the base space $L G / G$. This is the Borel-Weyl approach to representation theory where for each line bundle on a flag manifold we get an irreducible representations. In this case we get a representation of the loop group $L G$. Therefore if we consider a Riemann surface $\Sigma$ with $n$-punctures (Fig. 64) we can associate to each disc around the punctures a particular Hilbert space $\mathfrak{H}_{R i}$ corresponding to the representation $R_{i}$ of $L G$. This means that once we include the constraint (9.59) what we are obtaining is the set of invariants states in $\otimes_{i=1}^{n} \mathcal{K}_{R_{i}}$. For $\Sigma=S^{2}$ the set of invariants in $\bigotimes_{i=1}^{n} \mathcal{K}^{R_{i}}$ can be interpreted as the conformal blocks with external legs saturated by primmary fields in the representations $R_{i}$ :

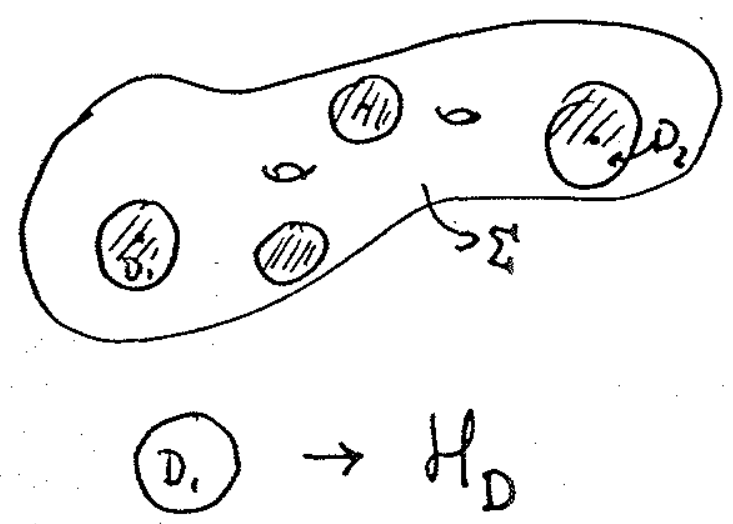

Fig. 64. The Hilbert space associated to a disc. 
From $(9.60)$ we get $^{33}$

$$
\begin{array}{ll}
\operatorname{dim} \nvdash\left(S^{2} ; R\right) & =0 \quad R \neq i d . \\
\operatorname{dim} \nvdash\left(S^{2} ; R_{i}, R_{j}\right) & =\delta_{i, j} \\
\operatorname{dim} \nvdash\left(S^{2} ; R_{i}, R_{j}, R_{k}\right) & =N_{i j}^{k} .
\end{array}
$$

This result, already mentioned in the chapter on quantum groups indicates a deep connection between Gauss's law (9.59) and the fusion algebra of the WZW models.

After these preliminaries, we define Knot invariants. We know that the space of conformal blocks in WZW models are irreducible representations of the braid transformations. From this fact and from (9.60) we can easily get a Skein rule.

Consider for instance the case of Fig. 65 and for simplicity take the group to be $\mathrm{SU}(2)$. Choose arbitrary a crossing point and consider it as living inside a 3-ball. The common boundary is $S^{2}$. If on the knot we have running a "quark" in the fundamental representation then the Hilbert space at the boundary will be $k\left(s^{2} ; 1 / 2 \ldots 1 / 2\right)$. For $k>1$ we know that $K_{\left(s^{2} ; 1 / 21 / 21 / 21 / 2\right)}$ is a two dimensional space and therefore a representation space of the Hecke algebra. The expectation value of the Wilson line on $k$ will be:

$$
\left\langle 1 / 2 \ldots 1 / 2 S^{2} ; M_{1} \mid M_{2} ; S^{2} ; 1 / 2 \ldots 1 / 2\right\rangle
$$
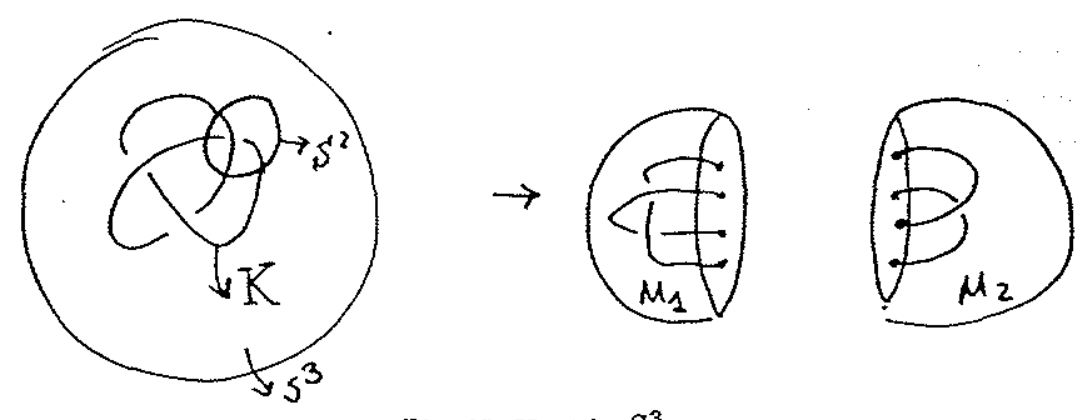

Fig. 65. Knot in $S^{3}$.

Now the Skein rule can be easily obtained using (9.6) and the representation of Hecke on $\left.H_{(} S^{2} ; 1 / 2 \ldots 1 / 2\right)$ (Fig. 66). To finish this section we will folds. The main ingedient for performing these co folds. The main ingredient for performing these computations is surgery. It is well-known that any three manifolds can be obtained by repeated surgery on Knots in $S^{3}$. The procedure consists of cutting a solid torus around the Knot and to glue it back after a diffeomorphism on its boundary. The partition function for the new manifold can be obtained as follows. First we divide $S^{3}$ in two pieces $M_{1}, M_{2}$, with $M_{1}$ the solid torus around the knot Fig. 67. This defines two states $\left|M_{1} ; \partial M_{1}\right\rangle\left|M_{2} ; \partial M_{2}\right\rangle$. Second, we perform a diffeomorphism $\rho$ on the boundary of the solid torus. This diffeomorphism can be represented by an operator acting on $H_{\partial M_{1}}$, say $K_{\rho}$. The new partition function is given by $\left\langle M_{2} ; \partial M_{2}\left|K_{\rho}\right| M_{1} ; \partial M_{1}\right\rangle$.

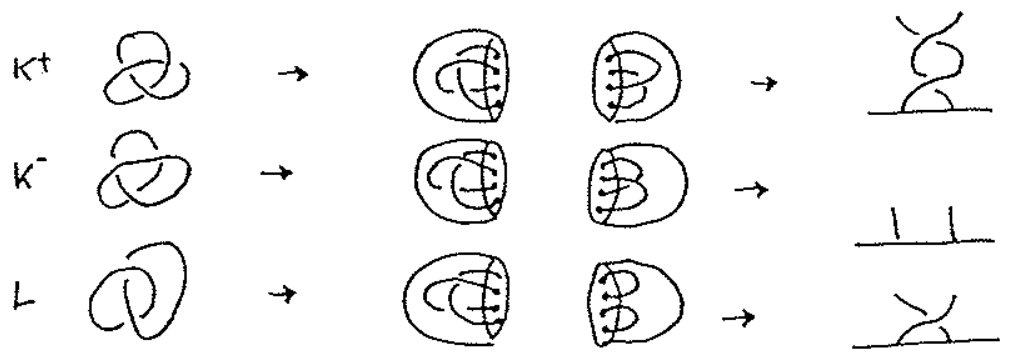

Fig. 66. The Skein rule for the trefoil. The r.h.s are the corresponding states in $H_{\left(S^{3} 1 / 2 \ldots 1 / 2\right)}$

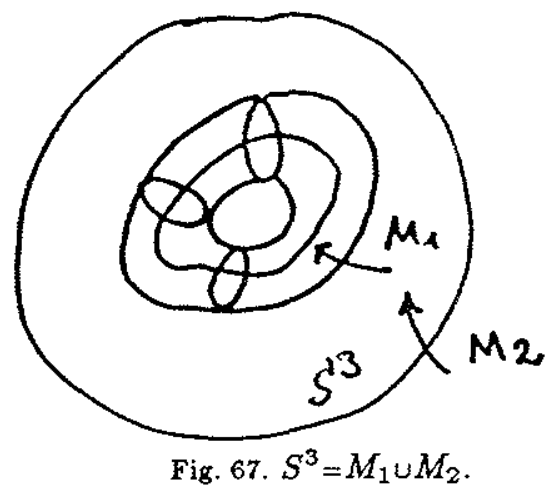

Let us consider as an example the two 3-manifolds $S^{3}$ and $S^{2} \times S^{1}$. These two manifolds can be obtained by gluing two solid torus. For $S^{3}$ this is the case presented in Fig. 67. $S^{1} \times S^{1}$ can also be obtained by gluing two solid torus. If we represent the solid torus by $D \times S^{1}$ with $D$ a disc, then $S^{2} \times S^{2}$ obtained by gluing the two discs through the boundary. (see Fig. 68). 


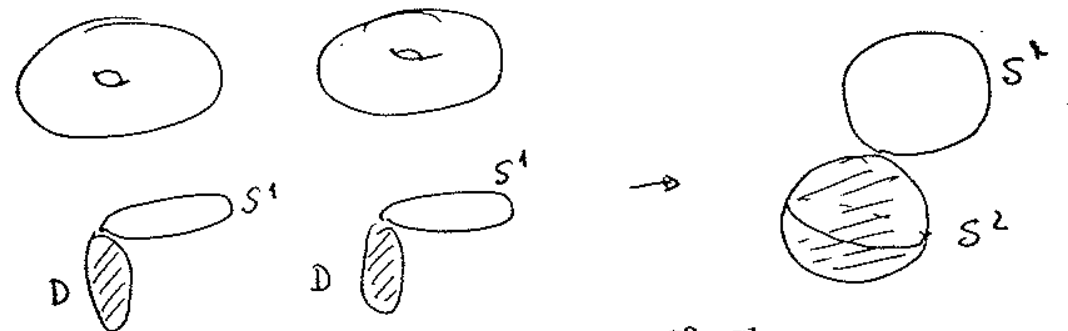

Fig. 68. Surgery rules to get $S^{2} \times S^{2}$.

To get $S^{2} \times S^{1}$ from $S^{3}$ we first consider the unknot in $S^{3}$, relative to this unknot we divide $S^{3}$ in two solid torus and we glue them back again after a modular transformation $\tau \rightarrow-1 / \tau$ on the boundary of the solid torus that it is surrounding the unknot. To compute the partition function we need to represent modular transformations on $\mathcal{K}_{T}$, the Hilbert space of a torus. A basis of $K_{T}$ is given by the set of character $K_{i}$ of the WZW model. The state $\left|M_{1} ; T\right\rangle$ will be identified with $\mathcal{K}_{0}$ the character of the vacuum. If inside $M_{1}$ we have a Wilson living in a representation $R_{i}$ the corresponding state will be $\mathfrak{H}_{i}$. Using the representation of modular transformations on the space of characters we get that the partition function on $S^{3}$ is $\Sigma_{i} S_{o i} Z\left(S^{2} \times S^{1} ; R_{i}\right)$ with $S$ the modular transformation matrix, and $z\left(S^{2} \times S^{1} ; R_{i}\right)$ is the partition function of $S^{2} \times S^{1}$ with one puncture in $S^{2}$ in the representation $R_{i}$. (see Fig. 69) From these surgery manipulations we can get an interesting expression for the invariant polynomials in $S^{3}$. In fact consider any link in $S^{3}$. Divide now $S^{3}$ in two solid tori in such a way that the link is contained in one of these Tori. Now cut the second torus away and glue it back again after a modular transformation. What one obtains is $S^{2} \times S^{1}$ with the knot represented by a set of braiding transformations and one spectator Wilson line that is not braided with the original knot. More explicitly one obtains the following representation of the link invariant:

$$
\sum_{i} S_{0 i} Z\left(S^{2} \times S^{1} ; \alpha, R_{i}\right)
$$

where $\alpha$ is the braiding transformation defining the original knot. (see Fig. 70).

Equation (9.63) is very interesting. In fact we can compute $Z\left(S^{2} \times\right.$ $\left.S^{1} ; \alpha, R_{i}\right)$ by simply computing the trace of the representation of the braiding $\alpha$ on $\nVdash\left(S^{2} ; R_{1} \ldots R_{n} ; R_{i}\right)$; the braid group $\alpha \in B_{n}$. From (9.60) this is equivalent to performing the trace of element $\alpha$ in the space of conformal
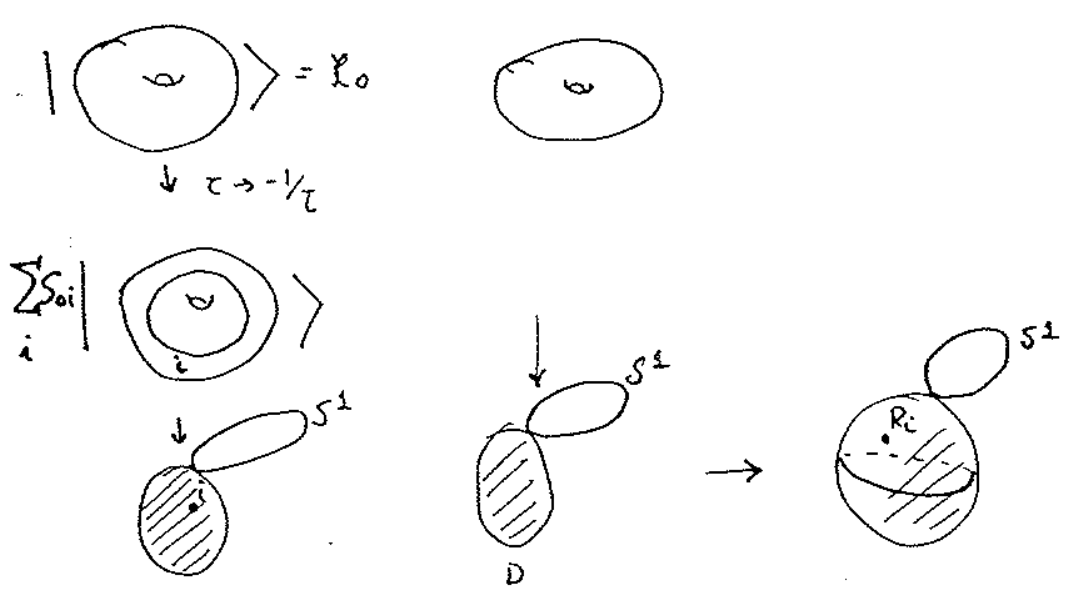

Fig. 69. $S^{2} \times S^{1}$ with an insection in the representation $R_{i}$.
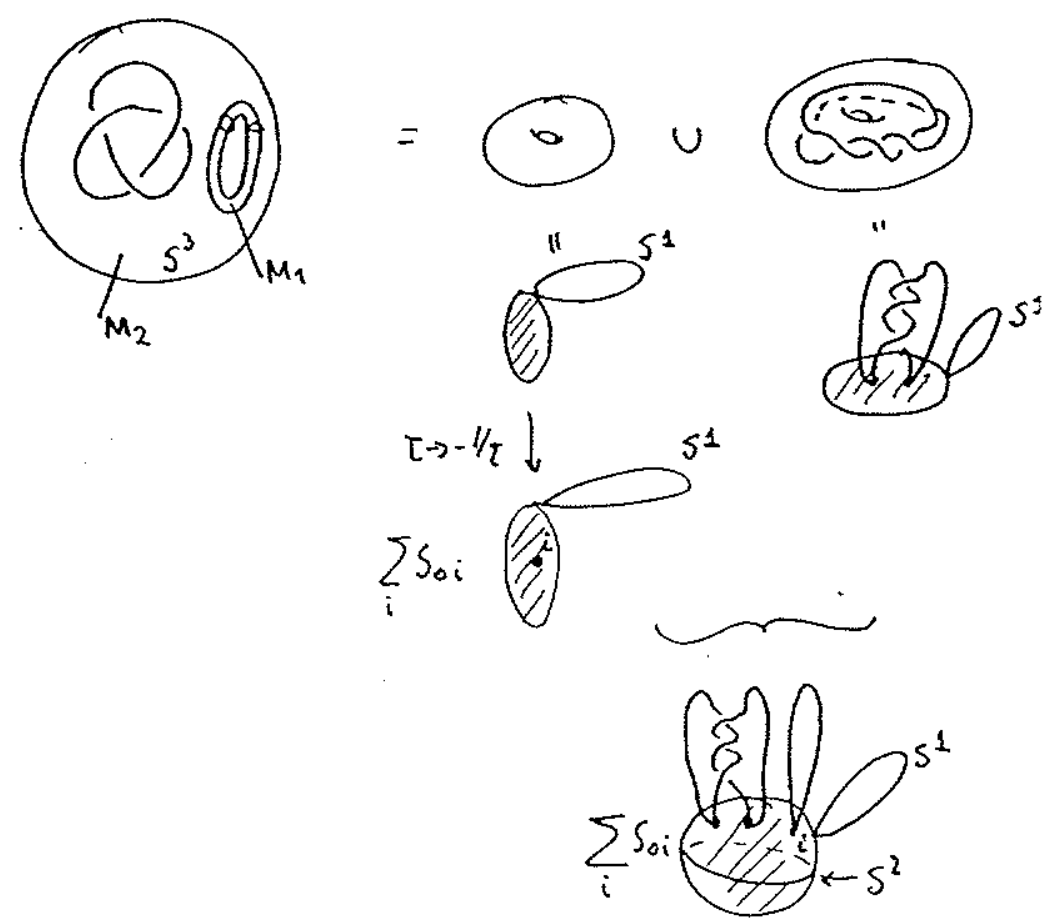

Fig. 70. Knots from $S^{3}$ to $S^{2} \times S^{1}$. 
blocks with $n+1$ external legs, the last one in the representation $R_{i}$. For simplicity let us assume all the representations $R_{1} \ldots R_{n}$ are equal to the fundamental representation. In this case we obtain

$$
Z\left(S^{2} \times S^{1} ; \alpha ; R_{i}\right)=\operatorname{Tr}\left[B_{(\alpha)}\right]_{\mathcal{H}\left(S^{2} ; 1 / 2 \ldots 1 / 2 ; R_{i}\right)}
$$

where $B(\alpha)$ is the representation of $\alpha \in B_{n}$ in the space of conformal blocks

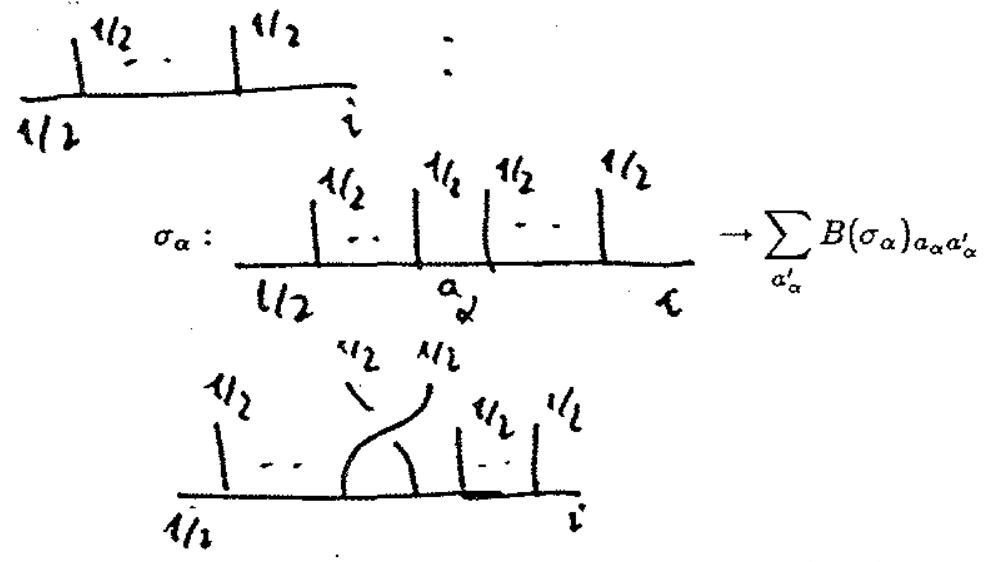

After these considerations we can come back to $\mathrm{Eq} .(9.24)$ and compare it with (9.63). We have the natural identification of $W_{j}$ with $S_{0 j}$. Normalizing (9.63) with respect to the partition function on $S^{3}$, we obtain:

$$
\frac{1}{S_{00}} \sum_{i} S_{0 i} Z\left(S^{2} \times S^{1} ; \alpha, R_{i}\right)
$$

and therefore we expect

$$
W_{t}=\frac{S_{0 j}}{S_{00}}
$$

For $\mathrm{SU}(2)_{k}$ WZW model the value of $\frac{S_{0 j}}{S_{00}}$ is $[2 j+1]_{q}$ with $q=e^{2 \pi i / k+2}$. This is precisely the value we get for the expansion of the Jones (or Turaev) polynomial in the expansion (9.24). This result is extremely interesting and indicates that the modular properties and the fusion algebra of the conformal field theory are deeply related with the Markov property of the traces used in the definition of invariant polynomials.

This concludes our brief account of Witten's approach to knots on arbitrary 3-manifolds. ${ }^{33}$

\section{References}

1. P. Ginsparg, eds. Les Houches Summer School 1988, Z. Brezin and J. ZinnJustin.

2. J. Cardy, eds. Les Houches Summer School 1988, E-Brezin and J. ZimJustin.

3. M. Green, J. Schwarz and E. Witten, String theory (Cambridge University Press, 1986), Vols. I and II.

4. M. Kaku, String theory (springer Verlag, 1988).

5. D. Lüst on S. Theisen Lectures and String Theory. Lectures Notes in Physics. (Springer-Verlag, 1989).

6. A. Belavin, A.M. Polyakov and A. Zamolodchikov, Nuel Phys. B241 (1984) 33.

7. J. Birman, Links, Braids and Mapping Class Groups (Princeton University Press, 1974).

8. D. Friedan, Z. Qiu and S. Shenker, Phys. Rev. Lett. 51 (1984) 1575.

9. V.S. Dotsenko and V.A. Fateev, Nucl. Phys. B240 (1984) 312; ibid. B251 (1985) 691, Phys. Lett. 154B (1985) 291.

10. A. Rocha-Caridi, in Vertex Operators in Math Physics.

11. E. Witten, Commun. Math. Phys.92 (1984) 83.

12. V.I. Knizhnik and A-Zamolodchikov, Nucl. Phys. B247 (1984) 83

13. D. Gepner and E. Witten, Nucl. Phys. 278B (1986) 493

14. A. Cappelli, C. Itzykson and J.B. Zuber, Commun. Math. Phys. 113 (1987)

15. E. Verlinde, Nucl. Phys. B300 (1988) 360.

16. G. Moore and N. Seiberg, Phys. Lett. $212 B$ (1988) 451.

17. G. Moore and N. Seiberg, "Naturalness in conformal field theories", IASSNS-HEP-88/31.

18. G. Moore and N. Seiberg, "Classical and quantum conformal field theories", IASSN-HEP-88/35.

19. C. Vafa, Phys. Lett. B206 (1988) 421.

20. G. Anderson and G. Moore, Commun. Math. Phys. 117 (1988) 441.

21. D. Friedan and S. Shenker, Nucl. Phys. B281 (1987) 509.

22. L. Alvarez Gaume, C. Gomez and G. Sierra, Nucl. Phys. B, to appear.

23. L. Alvarez Gaume, C. Gomez and G. Sierra, Phys. Lett. 220 (1989) 142.

24. G. Moore and N. Yu. Reshetikhin "A comment in quantum groups symmetry in conformal field theory", IASSNS-HEP-89/18.

25. L. Alvarez Gaume, C. Gomez and G. Sierra, "Duality and quantum groups", CERN TH 5369-89. 
26. V.G Drinfel'd, Sov. Math. Doke. 82 (1985) 254; M. Jimbo, Lett. Math. Phys. 10 (1985) 63; 11 (1986) 247.

27. V.G. Drinfel'd, Quantum Groups; Berkelg Col 1986.

28. N. Yu. Reshetikhin, "Quantized universal enveloping algebras and invariants of links", I,II LOMI-3-4-87, E-17-87.

29. A.N. Kirillov and N. Yu. Reshetikhin, "Representations of the algebra $\mathrm{Uq}(\mathrm{SL}(2 j)$ qorthogonal polynomials and invariant of "links", LOMI-E-988.

30. E. Witten, "Gauge theories, vertex models and quantum groups", IASSNSHP-89/32.

31. G. Lusztig, Modular representations of quantum groups", MIT preprint 1988.

32. V. Pasquier and H. Saleur, "Common structures between finite systems and conformal field theories through quantum groups ${ }^{\star}$, Saclay-SPHT-89/031.

33. E. Witten, Commun. Math. Phys. 121 (1989) 351.

34. R. Baxter, Exactly Solved Models in statistical Mechanics (Academic Press, 1982).

35. V. Pasquier, Nucl. Phys. B 285 (FS19) (1987) 162; J.Phys. A 20 (1987) 5707.

36. R. Baxter, Proc. Royal Society 289A (1978) 1359.

37. J. Rolisen, Knots and Links (Publish or Perish, Boston, 1976).

38. L. Kauffman, On Knots (Princeton University Press) 1988.

39. V.G. Tureev, Invent. Math. 92 (1988) 527.

40. V. Jones, Invent. Math. 72 (1983) 1.

41. V. Jones, Ann. Math 126 (9187) 335.

42. S. Ferrara, A. Grillo and R. Gatto, Nuovo Cimento 12 (1972) 959.

43. V.A. Fatteev and A. Zamolodchikov, Nucl. Phys.B 280 [FS 18] (1987) 644.

44. M. Berhadsky, V. Knizhrik and M. Teitelman, Phys. Lett. B 151 (1985) 31.

45. P.Di Vecchia, V.G. Knizhnik, J.L Petersen and P. Rossi, Nucl. Phys. B 253 (1985) 701 .

46. V. A. Fatteev and Lykiarnov.

47. A. Zamolodchikov, Montreal Lectures 1988; A. Gerasimov, A. Marshakov, A. Morozov, M. Olshanetsky and S. Shatashvili, ITEP preprint 1989; J. Distler and Z. Quio, CLNS 89/911; D. Nemeschansky, USC-89/012.

48. P. Goddard and D. Olive, Int. J. Mod Phys A1 (1986) 1.

49. V. Kac, Infinite Dimensional Algebras (Cambridge University Press).

50. D. Goss, J. Harvey, E. Martinec and R. Rohm, Nucl. Phys. B 256 (1985) 253 and Nucl. Phys. B 267 (1985) 75.
51. J. Cardy, Nucl. Phys. B270 [FS 16] (1986) 186.

52. A. Capelli, C. Itzykson and J.B. Zuber, Nucl. Phys. B 280 (1987) 445 and Commun. Math. Phys. 113 (1987) 1.

53. R. Dijkgraaf and E. Verlinde, "Fusion algebras and conformal field theories", centribution to the Annecy Conference on Conformal Field theory, 1988.

54. P. Bowknegt and W. Nalim, Phys. Lett, 184 B (1987) 359.

55. C. Itzykson, Proceedings of Annecy Workshop; Nucl. Phys. 5B (1988) 150.

56. B. Schellekens and S. Yankielowicz, CERN TH 5344/89.

57. R. Dijkgraaf, E. Verlinde and H. Verlinde, Commun. Math. Phys. 115 (1988) 649.

58. T. Kawai, University of Tokyo Komaba preprint 21340 (88).

59. H. Wenzl, Invent. Math. 92 (1988) 349.

60. G. Felder, J. Fröhlich and G. Keller, ETH preprint (February 1989).

61. A. Tsuchiya and Y. Kanie, Lett. Math. Phys. 13 (1987) 303.

62. J. Lacki and P. Zaugg, Geneve University preprint 1989.

63. R. Baxter, Aun. Phys. 70 (1972) 193.

64. A. Zamolodchikov, Sov. Phys. Rev. 2 (1980).

65. G.F. Andrews, R. J. Baxter and P.J. Forrester, J. Stat Phys. $\mathbf{3 5}$ (1984) 193 E. Date, M. Jimbo, A. Kuniba, T. Miwa and M. Okada, Nucl. Phys. 240 [FS 17] 1987 (231), Adv. Stud. Pure Math. 16 (1987).

66. P. Freyd, d. Yetter, J. Horte, W. Lickorish, K. Millet and A. Ocneanu, Bull A MS 12 (1985) 239.

67. L. Kauffman, Topology 26 (1987) 395

68. G. Segal; Atiyah-Semminar 1989. and paper in preparation. 